\title{
Context-based image analysis
}

Citation for published version (APA):

Bergboer, N. H. (2007). Context-based image analysis. [Doctoral Thesis, Maastricht University]. Universiteit Maastricht. https://doi.org/10.26481/dis.20071010nb

Document status and date:

Published: 01/01/2007

DOI:

10.26481/dis.20071010nb

Document Version:

Publisher's PDF, also known as Version of record

\section{Please check the document version of this publication:}

- A submitted manuscript is the version of the article upon submission and before peer-review. There can be important differences between the submitted version and the official published version of record.

People interested in the research are advised to contact the author for the final version of the publication, or visit the DOI to the publisher's website.

- The final author version and the galley proof are versions of the publication after peer review.

- The final published version features the final layout of the paper including the volume, issue and page numbers.

Link to publication

\footnotetext{
General rights rights.

- You may freely distribute the URL identifying the publication in the public portal. please follow below link for the End User Agreement:

www.umlib.nl/taverne-license

Take down policy

If you believe that this document breaches copyright please contact us at:

repository@maastrichtuniversity.nl

providing details and we will investigate your claim.
}

Copyright and moral rights for the publications made accessible in the public portal are retained by the authors and/or other copyright owners and it is a condition of accessing publications that users recognise and abide by the legal requirements associated with these

- Users may download and print one copy of any publication from the public portal for the purpose of private study or research.

- You may not further distribute the material or use it for any profit-making activity or commercial gain

If the publication is distributed under the terms of Article $25 \mathrm{fa}$ of the Dutch Copyright Act, indicated by the "Taverne" license above, 


\section{Context-Based Image Analysis}

Niek Henri Bergboer

October 10th, 2007 

Context-Based Image Analysis 



\title{
Context-Based Image Analysis
}

\author{
PROEFSCHRIFT
}

ter verkrijging van de graad van doctor

aan de Universiteit Maastricht, op gezag van de Rector Magnificus, Prof. mr. G.P.M.F. Mols,

volgens het besluit van het College van Decanen, in het openbaar te verdedigen op woensdag 10 oktober 2007 om 16:00 uur

door

Niek Henri Bergboer 
Promotores: $\quad$ Prof. dr. H. J. van den Herik

Prof. dr. E. O. Postma

Leden van de beoordelingscommissie:

Prof. dr. A. J. van Zanten (Voorzitter)

Prof. dr. R. Goebel

Prof. dr. P. Lambin

Prof. dr. T. M. Mitchell (Carnegie Mellon University)

Prof. dr. ir. A. Nijholt (Universiteit Twente)

\section{$\widehat{N W O}$}

Netherlands Organisation for Scientific Research

The research reported in this thesis has been funded by NWO in the framework of the ToKeN project EIDETIC, grant number 634.000.001.

\section{Dissertation Series No. 2007-14}

The research reported in this thesis has been carried out under the auspices of SIKS, the Dutch Research School for Information and Knowledge Systems.

ISBN 978-90-64641-64-0

(C)2007 N.H. Bergboer, Maastricht, The Netherlands.

Cover design: Niek Bergboer

The cover image shows a computer-rendered left brain hemisphere. A small inner portion of a human face is overlaid on the so-called fusiform face area; an area in the brain believed to be involved in face detection and identification. The use of only the inner portion of the face illustrates the importance of familiair context in finding faces. The face is that of Thomas Bayes; the founding father of modern-day statistics. The illustration was made using images that are either created by the author of this thesis, or images that are in the public domain.

All rights reserved. No part of this publication may be reproduced, stored in a retrieval system, or transmitted, in any form or by any means, electronically, mechanically, photocopying, recording or otherwise, without prior permission of the author. 


\section{Preface}

For the final two years of my applied physics studies, I chose a master in the field of modelling and system identification. After an additional year of research work in the system identification field, I decided to broaden my scope, and to apply for a Ph.D. position. In Maastricht, I was offered a Ph.D. position that had a combination of two interesting features. First, the project centred around image analysis, and second, the emphasis of the project was on machine-learning techniques. The latter was a natural fit given my previous modelling experience.

In the early stages of the project, I became intrigued, together with my supervisors, in the use of contextual information. My research concentrated on finding objects in images, and, later, on finding task-specific brain areas in fMRI data. The research led to a number of conference publications, two journal publications, and this thesis. Many people have, directly or indirectly, contributed to this thesis. In the remainder of this preface, I would like to thank them.

First and foremost, I would like to acknowledge my supervisors Eric Postma and Jaap van den Herik, for their guidance, whenever I lost myself in technical details. Their tireless and valuable feedback have greatly improved the quality of this thesis. Without the help of both of them, this thesis would never have appeared. In addition, I would like to recognise Elia Formisano and Federico de Martino, from the Department of Neurocognition. The invaluable cooperation with them made that the fMRI-related part of this thesis received so much approval.

Then, I would like to thank my colleagues and former colleagues for providing a nice working atmosphere, and for helping me reflect on the significance of my work. In particular, I am grateful to my roommates Guido, Igor, and Laurens, for enduring my singing, and for our many discussions on a sometimes alarmingly unlimited range of subjects. Moreover, I would like to express my gratitude towards Joke Hellemons, Marlies van der Mee, Hazel den Hoed, and Tons van den Bosch, for their help with administrative matters. Finally, I would like to thank our systems administrator Peter Geurtz, for our pleasant conversations, and for allowing me all the ICT-related freedom that I enjoyed.

While a Ph.D. project provides the Ph.D. student the opportinity to growth in the scientific world, my supportive environment gave me many other equally important opportunities for a grow in personality. In this regard, I am grateful to my friends for being there whenever I needed a break. In particular, I would like to mention the members of the Maastricht University Choir. I gratefully remember our singing together, and the many memorable social events we enjoyed. 
Furthermore, I am thankful to the members of the Limburg Racing Team, for the fun and exciting motorbike tours we made together.

In the personal sphere, I would like to thank my parents and my sister, for their solid support over the years. Finally, I would like to address Vivianne. Her support and inspiration encouraged me to continue my research, and to overcome any obstacle in the world despite our frequent geographical separation and common Ph.D. stress over the last year. Such encouragement can only be given by the love of your life. Thank you, Vivianne!

Niek Bergboer, 2007

\section{Acknowledgements}

This research is funded by NWO, the Netherlands Organisation for Scientific Research, within the ToKeN project EIDETIC (grant number 634.000.001). The research has been carried out under the auspices of SIKS, the Dutch Research School for Information and Knowledge Systems. Moreover, I gratefully acknowledge financial support over the years by the Universiteitsfonds Limburg / SWOL. 


\section{Table of Contents}

Preface $\quad$ v

Table of Contents vii

List of Figures xii

List of Tables xiii

1 Introduction 1

1.1 Image and context ... . . . . . . . . . . . . . . . . 2

1.2 Problem statement . . . . . . . . . . . . . . . . 2

1.3 Application domain and research questions . . . . . . . . . . 3

1.3.1 Detection of natural objects . . . . . . . . . . . . 3

1.3.2 Detection of task-specific brain regions . . . . . . . . . . 4

1.4 Methodology .................... 5

1.5 Thesis outline ....................... 6

I Object Detection 9

2 Objects and their contexts 11

2.1 The cultural-heritage problem statement . . . . . . . . . . 11

2.2 The use of context for object detection . . . . . . . . . . . . . 13

2.2.1 The combination . . . . . . . . . . . . . . 14

2.2 .2 The definition . . . . . . . . . . . . . . . . . . . . . . . . . . . . . . . .

2.3 Datasets in human-face detection . . . . . . . . . . . . . 14

2.3.1 Natural-image set . . . . . . . . . . . . . . . 14

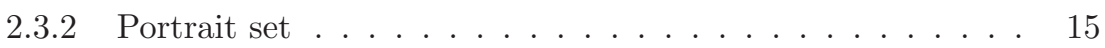

2.4 Performance criteria . . . . . . . . . . . . . . . . 15

2.5 Binary-classifier structures . . . . . . . . . . . . . . . . 17

2.5.1 The monolithic classifier . . . . . . . . . . . . . . 17

2.5.2 The boosted-cascade classifier . . . . . . . . . . 17 
3 Context-based object detection $\quad 19$

3.1 The context-detection method . . . . . . . . . . . . . . . . . 19

3.2 Context detection . . . . . . . . . . . . . . . . . 20

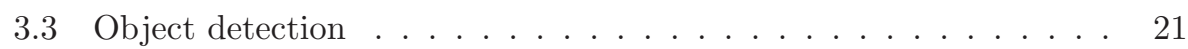

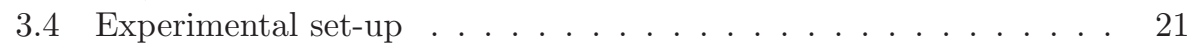

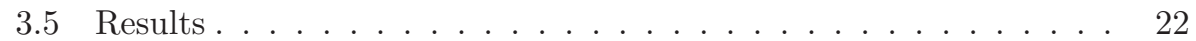

3.6 Discussion . . . . . . . . . . . . . . . . . . 24

3.6.1 General considerations on accuracy and speed . . . . . . . . 24

3.6.2 Small windows versus large windows . . . . . . . . . . 25

3.6.3 Related work . . . . . . . . . . . . . . . 25

3.7 Chapter summary . . . . . . . . . . . . . 26

4 Gradient-based context-based object detection $\quad 27$

4.1 Domain knowledge on relative locations . . . . . . . . . . . . 28

4.2 The gradient method . . . . . . . . . . . . . . . . 28

4.2.1 The object-detection stage . . . . . . . . . . . . . 29

4.2.2 The context-validation stage . . . . . . . . . . . . . 29

4.3 Algorithmic Approaches . . . . . . . . . . . . . . . . . . . 30

4.3.1 Object classifiers . . . . . . . . . . . . . . . . . . . 31

4.3.2 Relative localisation ................ . . 31

4.3.3 Estimating the PDF using cluster-weighted models . . . . . 31

4.3.4 Estimating the PDF using decision trees . . . . . . . . . . . 32

4.4 Experimental set-up: human-face detection . . . . . . . . . . . 33

4.4.1 Detection of face candidates . . . . . . . . . . . . 33

4.4.2 Contextual validation . . . . . . . . . . . . . 33

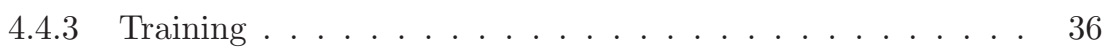

4.5 Evaluation procedure . . . . . . . . . . . . . . . . . . 40

4.6 Results . . . . . . . . . . . . . . . . . . . . . . . . . . . . . . . . .

4.6 .1 Observations . . . . . . . . . . . . . . . . 42

4.6.2 Small windows compared to large windows . . . . . . . . 44

4.7 Discussion . . . . . . . . . . . . . . . . . . . . 46

4.7.1 Detection accuracy and speed . . . . . . . . . . 46

4.7.2 Small versus large windows . . . . . . . . . . . . . . . . 46

4.7.3 Feature types and normalisation . . . . . . . . . . . 47

4.7.4 The "object-is-near" assumption . . . . . . . . . . . . . 49

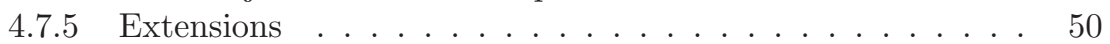

4.8 Chapter conclusions ..................... 53

5 General discussion of object detection $\quad \mathbf{5 5}$

5.1 The speed versus accuracy trade-off . . . . . . . . . . . . 55

5.2 Explaining the gradient method's performance . . . . . . . . . 56

5.2 .1 The use of local context . . . . . . . . . . . . 56

5.2 .2 The relative-localisation model . . . . . . . . . . . 57

5.2 .3 Training using relative locations . . . . . . . . . . . 58

5.3 Broader relevance for the cultural heritage . . . . . . . . . . . . 59

5.4 Related work ................... 60 
5.5 Future directions . . . . . . . . . . . . . . . . 62

5.5.1 Future research directions . . . . . . . . . . . . 62

5.5.2 Future application directions ............. 64

\section{Functional MRI Brain Mapping 65}

6 Spatiotemporal brain mapping on fMRI data $\quad 67$

6.1 The medical problem statement . . . . . . . . . . . . . 67

6.2 Functional MRI and brain representation ........... . . 69

6.2.1 Functional Magnetic Resonance Imaging (fMRI) . . . . . . 69

6.2.2 General data preprocessing . . . . . . . . . . . . . 70

6.2.3 Data selection................... . . . . 71

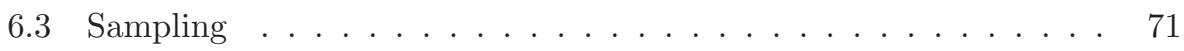

6.3.1 Temporal sampling . . . . . . . . . . . . 72

6.3.2 Spatial sampling: spherical coordinate indexing system . . 72

6.4 The region-based method . . . . . . . . . . . . . . 74

6.4.1 Definition of features . . . . . . . . . . . . . . . 74

6.4.2 The Bayesian algorithm . . . . . . . . . . . . . . . 77

6.4.3 Brain-state classification . . . . . . . . . . . . . . 80

6.4.4 Scale estimation ................ . . 81

6.5 Experimental set-up . . . . . . . . . . . . . . 82

6.5.1 Dependent and independent variables ......... 83

6.5.2 Evaluation criteria . . . . . . . . . . . . . . 86

6.6 Results....................... 86

6.6.1 Region-detection results . . . . . . . . . . . . . . 87

6.6.2 Scale-estimation results . . . . . . . . . . . . . . . 91

6.7 Challenging the use of simulated data . . . . . . . . . . . . . . . 95

6.8 Chapter conclusions . . . . . . . . . . . . . . . 96

$7 \quad$ Applying the region-based method to real data $\quad 99$

7.1 Data selection and preprocessing . . . . . . . . . . . . 100

7.1 .1 Data selection . . . . . . . . . . . . 100

7.1.2 Dataset properties ................ 101

7.1.3 Data preprocessing . . . . . . . . . . . . 101

7.2 Application of the region-based method to the dataset . . . . . . . 102

7.2.1 Sampling . . . . . . . . . . . . . . 102

7.2 .2 Feature selection . . . . . . . . . . . . . . 103

7.2 .3 Scale determination . . . . . . . . . . . . . . . . . 104

7.3 Experimental set-up . . . . . . . . . . . . . . . . 105

7.3.1 Experimental procedure . . . . . . . . . . . 105

7.3 .2 Performance indicators . . . . . . . . . . . . 105

7.4 Results......................... 106

7.4.1 Specific-region detection results . . . . . . . . . . . 107

7.4.2 Scale-estimation results . . . . . . . . . . . . 110

7.5 Discussion . . . . . . . . . . . . . . . . 113 
7.5.1 Inter-subject variations . . . . . . . . . . . . . . . . 113

7.5.2 Scale calculation . . . . . . . . . . . . . . . . 114

7.5.3 Choice of brain hemisphere . . . . . . . . . . . . 116

7.6 Chapter conclusions . . . . . . . . . . . . . . 117

8 General discussion of the region-based method $\mathbf{1 1 9}$

8.1 Sampling and noise issues . . . . . . . . . . . . . . . 119

8.1.1 Sampling and noise . . . . . . . . . . . . . . 119

8.1.2 Weighting for noise . . . . . . . . . . . . . . 120

8.2 Wavelet-feature issues . . . . . . . . . . . . . . . 121

8.2.1 Wavelet types . . . . . . . . . . . . . . . . . . . . . . . . . . . . . . . 123

8.2 .2 Wavelet basis . . . . . . . . . . . . . . . . 124

8.2.3 Wavelets and spatial smoothing . . . . . . . . . . . . . 124

8.3 The use of Bayesian classification . . . . . . . . . . . . . . . . . . . . . . . 125

8.4 Related work . . . . . . . . . . . . . . . . 125

8.4.1 Global spatial correlations . . . . . . . . . . . 126

8.4.2 Localised multivariate analysis . . . . . . . . . . . . . 126

8.4.3 Univariate analysis and spatial relations . . . . . . . . . . 127

8.4 .4 Feature selection . . . . . . . . . . . . . . 127

8.5 Future directions . . . . . . . . . . . . . . . . . . 128

8.5.1 Fully automated scale estimation . . . . . . . . . . . . 128

8.5.2 Extensions to multi-stimulus classification . . . . . . . . . 129

8.5.3 Application to other cortical-surface quantities . . . . . . 130

General conclusions and recommendations 131

9 Conclusions $\quad 133$

9.1 Answers to the research questions . . . . . . . . . . . . 133

9.1.1 The cultural-heritage domain . . . . . . . . . . . 133

9.1.2 The medical domain . . . . . . . . . . . . . . . 135

9.2 Answer to the problem statement . . . . . . . . . . . . . . . . 137

9.3 Two directions for future research . . . . . . . . . . . . . . . . 138

$\begin{array}{ll}\text { References } & 139\end{array}$

Appendices

A Performance considerations in Bayesian fMRI brain mapping $\quad 151$

A.1 Integral images and wavelet calculation . . . . . . . . . . . . . 151

A.1.1 Integral-image framework . . . . . . . . . . . . . . 151

A.1.2 Calculation of wavelets . . . . . . . . . . . . 153

A.1.3 Computational complexity . . . . . . . . . . . . . 156

A.2 Sorting and grouping of specificities . . . . . . . . . . . 157

$\begin{array}{ll}\text { Summary } & 159\end{array}$ 
Samenvatting 


\section{List of Figures}

2.1 Examples of patterns that are similar to faces. . . . . . . . . . . . 12

2.2 Detection grouping example. . . . . . . . . . . . . . . . . 16

2.3 Overview of a boosted-cascade classifier . . . . . . . . . . . 18

3.1 Graphical representation of the context-detection method. . . . . . 20

3.2 The three different context types used in the context-detection method. . . . . . . . . . . . . . . 21

3.3 Accuracies obtained with the context-detection methods . . . . . . 23

4.1 Graphical representation of the gradient method. . . . . . . . . . . 30

4.2 The region used for obtaining training samples, overlaid on a face. 34

4.3 Average wavelet features over the training region. . . . . . . . . . 35

4.4 The cluster centres for the Gaussian mixture models. . . . . . . . . 38

4.5 The 25 spatial relative location classes used for the decision tree. . 39

4.6 Receiver Operating Characteristic for both gradient methods. . . . 43

4.7 Receiver Operating Characteristic for small and large windows. . . 45

4.8 PCA components 1 to 16 for scale-2 horizontal wavelets. . . . . . . 47

4.9 Receiver Operating Characteristic for the decision-tree method. . . 50

4.10 ROC compared to the combined gradient method. . . . . . . . . . . 51

4.11 Receiver Operating Characteristic for scale 2-3 and null-class methods. .......................... 52

5.1 Detection results for the context detector. . . . . . . . . . . . . 57

5.2 Performance on the painting set. . . . . . . . . . . . . . 60

5.3 Detection example for a self portrait by Rembrandt (1669). . . . . 61

6.1 Euclidean and cortical-surface distances in the brain. . . . . . . . . 72

6.2 Temporal selection of features. . . . . . . . . . . . . . . . . 73

6.3 Spherical coordinate system. . . . . . . . . . . . . . . . . 74

6.4 Spherical wavelet types. . . . . . . . . . . . . . . . 76

6.5 Two different patterns of activation. . . . . . . . . . . . . 84

6.6 Signal-to-noise amplitude ratio and contrast-to-noise amplitude ratio. 85

6.7 ROC curve for the brain with unequally-sized regions. . . . . . . . 90

6.8 Scale mismatch for different feature fractions. . . . . . . . . . . . . 93

7.1 Left brain hemisphere mesh for one of the four subjects. . . . . . . 102

7.2 BOLD response in a block-design paradigm. . . . . . . . . . . . 103

7.3 Brain regions involved in discriminating faces from objects. . . . . 106

7.4 Brain specificity map obtained with the GLM. . . . . . . . . . . . 109

7.5 Brain specificity map obtained with the region-based method. . . . 111

7.6 Brain specificity map for the GLM on a single subject. . . . . . . . 115

7.7 Brain specificity map for the most specific gradient wavelets only. . 116

A.1 Integral images. . . . . . . . . . . . . . . . . . 154 
A.2 Calculating a portion of a type-2 wavelet in four steps . . . . . . 155

A.3 Extended triangle structure for the spherical coordinate system. . . 156

\section{List of Tables}

3.1 False detections per image at several detection rates. . . . . . . . 23

3.2 Comparison of times required for detection, for different image sizes. 24

4.1 Detections results for the cluster-weighted method . . . . . . . . 41

4.2 Detections results for the decision-tree method . . . . . . . . . . . 41

4.3 Detection results for large and small windows. . . . . . . . . . . . 45

4.4 Detection results for absolute feature normalisation methods. . . . 49

4.5 Detection results for non-absolute feature normalisation methods. . 49

4.6 Detection results when using wavelets at levels 2 and 3. . . . . . 51

4.7 Detection results for model with a null-class. . . . . . . . . . . . . 52

5.1 Performance on the painting set. . . . . . . . . . . 59

6.1 GLM results on the brain with equally-sized regions. . . . . . . . . 87

6.2 GLM results on the brain with unequally-sized regions. . . . . . . . 88

6.3 Detection-rate results for two activation patterns. . . . . . . . . 89

6.4 Scale-estimation results for two activation patterns. . . . . . . . . . 92

6.5 Optimised scale-estimation results for two activation patterns. . . . 94

7.1 Identification of important faces-objects brain regions. . . . . . . . 108

7.2 Scale estimates for important faces-objects brain regions. . . . . . 112

8.1 Detection-rate results using noise weighting . . . . . . . . . . 121

8.2 Region-scale estimation using noise weighting. . . . . . . . . . . . . 122

8.3 Detection-rate results using averaging wavelets. . . . . . . . . . 123

8.4 Scale-estimation results using averaging wavelets. . . . . . . . . 123 



\section{Chapter 1}

\section{Introduction}

The rapid growth of digitally stored images is accompanied by the equally fast development of image-analysis techniques. The past two decades have brought many powerful methods that solve a large variety of image-analysis problems. Overviews of the methods and techniques are provided by, e.g., Jain, Duin, and Mao (2000), Lillesand, Kiefer, and Chipman (2004), Martinez-Trinidad, Ochoa, and Kittler (2006). The main point, however, is that the reliability of the techniques is still an issue. Even the reliability of the methods is sometimes at stake. We give two examples: (1) the poor reliability of methods for the automatic detection of objects in images frequently leads to false detections (Hjelmås and Low, 2001; Bashir and Porikli, 2006), and (2) the detection of task-specific regions in brain images acquired with functional magnetic resonance imaging (fMRI) is still far from perfect. The limited reliability in these domains leads to the classification of isolated brain locations as task specific, whereas these locations are not task specific in reality (Holmes, Poline, and Friston, 1997; Lange, Meyer-Bäse, and Wismüller, 2006).

In this thesis, we ${ }^{1}$ combine image-analysis and machine-learning methods in an attempt to improve the reliability of image-analysis methods, by using knowledge about the structure of images. This knowledge takes mostly the form of statistical regularities within the local spatial context of an image region of interest. We apply our methods in two application domains: (1) object detection in natural images, and (2) task-specific brain mapping in functional MRI images.

In Section 1.1 we provide our definitions of image and context. The main problem statement is stated in Section 1.2. The problem statement is applied to the two application domains mentioned above. These application domains, together with the research questions, are introduced in Section 1.3. The methodology employed in addressing the research questions is described in Section 1.4. We conclude this chapter by providing an outline of the thesis in Section 1.5.

\footnotetext{
${ }^{1}$ The plural "we" is used in this thesis, in recognition of the fact that research is an inherently collaborative effort.
} 


\subsection{Image and context}

The concept of "image" is very broad. The Oxford Dictionary has eight definitions of "image" (Soanes and Hawker, 2005). In this thesis, the concept of image is used in a much more confined way. We define an image as a spatially organised set of values in which a certain quantity varies smoothly with the location in space. Apart from the natural images and the fMRI images that we consider in this thesis, examples of images are radar images, in which the electromagnetic echo magnitude varies with the position, and remote-sensing images, in which the magnitude of several measured quantities varies with the position on the surface of the earth.

The Oxford Dictionary defines "context" as "the circumstances that form the setting for an event, statement, or idea.", or "the parts that immediately precede and follow a word or passage and clarify its meaning." As such, this definition conforms to the intuitive notion that the context of an entity provides information about that entity, in addition to the information that would be provided by the entity in isolation. As the above definition is too broad for our purposes, we define context in the domain of images as the local spatial context surrounding an image region of interest. Our rationale for choosing this definition of context, is that images contain structural information that extends beyond the object of interest. For instance, it is known that natural images have statistical regularities such as local spatial dependencies on the intensity values of the image (Baddeley and Hancock, 1992). The autocorrelation function, which measures spatial dependencies, falls slowly with distance (Simoncelli and Olshausen, 2001; Torralba and Oliva, 2003). The local correlation of intensity in natural images is caused by the spatial extent of (1) an object or (2) homogeneously coloured, or textured, surfaces in the visual scene. In artificial images, such as fMRI images, local correlations may result from diffusion processes. In the brain, cortical neurons with similar sensitivities tend to be spatially clustered and interconnected (Forman et al., 1995). Hence, stimulus-specific activations tend to diffuse over an extended region. The two examples suggest that the local spatial context of an image region provides cues about the intensity values at that region.

\section{$1.2 \quad$ Problem statement}

The above considerations on image analysis and the use of local-contextual information enables us to state the general problem statement of this thesis.

Problem statement: How can information from a local spatial context be utilised to improve the performance or reliability of imageanalysis techniques?

In general, the exact definition of (1) "local spatial context", (2) the nature of the information extracted from it, and (3) the measure of performance, cannot be given, as these are too specific to the application domain.

In addressing the problem statement, we confine our research to two application domains: the cultural-heritage domain and the medical domain. Within these domains, we focus on the following applications: (1) the detection of objects 
in natural photographic images, and (2) the detection of task-specific regions in functional MRI images. These applications, together with their associated research questions and the definition of reliability or performance, are described in Section 1.3.

\subsection{Application domain and research questions}

In the next two subsections we introduce the two domains, viz. the culturalheritage domain and the medical domain. Subsequently, we provide a condensed overview of the problem statement tailored to the domains. Finally, we state the research questions following from the domain-specific problem statement.

\subsubsection{Detection of natural objects}

In the cultural-heritage domain we investigate the use of the local spatial context in natural and painted images, in an attempt to improve the object-detection reliability. Object detection is an automatic procedure in which the computer searches and finds objects from a given class in (a set of) images, and returns a list containing the positions and sizes of the objects.

The problem statement tailored to the cultural-heritage domain is stated as follows.

Domain-specific problem statement: To what extent can the local spatial context of an object enhance the reliability of object detection in natural and painted images?

Reliability is a task-dependent metric, and needs to be defined for the specific case of object detection. In Part I of the thesis, we will take the number of false detections as an indicator of reliability. In particular, we will try to use the local spatial context of an object in order to reduce the number of false detections. Our first research question thus becomes as follows.

Research question 1 (RQ1): How can local spatial context be used to lower the number of false detections (i.e., achieve a more accurate detection) at an acceptable speed in automatic object detection?

As it is not known a priori which size of the context region around the object yields to most useful information, we pose a second research question.

Research question $\mathbf{2}$ (RQ2): What size of the context region is best suited to lower the false-detection rate?

We aim at presenting and evaluating novel algorithms that incorporate a local context to achieve reliable object detection. 


\subsubsection{Detection of task-specific brain regions}

In the medical domain we investigate how the use of local spatial context improves a brain-mapping performance based on functional MRI data. Brain mapping is the automatic determination of the location and the size of task-specific brain regions based on functional MRI data obtained in an experiment in which the subject is exposed to distinct stimuli. Here, the local spatial context consists of a region surrounding task-specific brain areas. The problem statement specific to the medical domain reads as follows.

Domain-specific problem statement: To what extent can local context enhance the performance of detecting task-specific regions of activity in the brain?

In the second part of the thesis, the region-detection performance will be expressed in terms of "specificity" and "scale" of the activated region. A novel method named the "region-based method" will be introduced there. The performance will be particularly assessed by four independent characteristics that can be quantified reasonably well. First, a method is good if it successfully marks all task-specific brain areas as "specific", while marking none of the non task-specific brain areas as "specific". This leads to the following research question.

Research question 3 (RQ3): Can a new region-based method detect brain regions with high specificity on a level comparable to or better than the standard method used in the field?

The second characteristic on which performance can be measured, is the scale of task-specific regions. Existing methods do not provide an independent scale estimate, whereas our method does. This leads to the following research question.

Research question 4 (RQ4): Can a new method deliver a reliable scale estimate for specific regions?

Both the first and the second characteristics can be measured on simulated brain data. In Chapter 6, we will perform such measurements, as the use of simulated data enables one to obtain quantitative results. However, it is difficult to evaluate a method when testing on simulated data only; the reasons are pointed out in Chapter 6.

Therefore, the third characteristic on which performance can be measured is whether a method's performance generalises well from simulated data to real data. This leads to the following research question.

Research question 5 (RQ5): Does the applicability of the new method generalise from simulated activation data to real data?

The fourth characteristic on which performance can be measured, is the robustness of a method with respect to interpersonal anatomical differences. In an experiment involving multiple test subjects, the anatomical differences among the subjects make the problem of brain mapping harder for current methods. This leads to the final research question. 
Research question 6 (RQ6): Can the region-based method cope with interpersonal differences in anatomy in a better way than standard methods?

\subsection{Methodology}

In this section we present the methodology used to answer the problem statement, and the research questions. In both our application domains, we employ machinelearning techniques to learn the relation between context and object or between context and region of interest. Machine learning is a powerful methodology to derive statistical relations between a set of example patterns and their associated desired outcome (class, or value). The relations are then hoped to generalise well to new patterns. In order to apply machine learning we need a set of example patterns, which is domain-specific. Therefore, in both application domains, the acquisition of a dataset is an integral part of the methodology.

In order to answer research question RQ1, we use a research methodology that consists of four phases; only thereafter we start applying our context-based methods. First, we perform a literature survey, in order to determine to which currently existing object-detection methods we can compare our method. Second, we define what region in an image, with respect to the object location, we regard as local spatial context. Third, we define precisely what performance criteria we use, e.g., what is a detection, and a false detection. Fourth, as stated in the previous paragraph, we acquire one or more datasets to train and test machine-learning methods. These four phases are covered in Chapter 2. Subsequently, in Chapter 3, we develop a context-based object-detection method. We describe the method in detail, and set up an experimental comparison between a currently existing object-detection method, and our context-based object-detection method. This comparison is based on the datasets, context regions, and performance criteria established earlier. The analysis of the first context-based object-detection method results in an additional research question RQ1a, and forms the starting point for the next context-based object-detection method in Chapter 4. The second contextbased object-detection method is analysed in a comparable manner as the first one.

In order to answer research question RQ2, we carry out the experiments alluded to above, using different context sizes. A comparison of the results, based on the datasets and performance criteria established earlier, allows us to determine which size of the context region yields the best results.

In order to answer research question RQ3, we have three phases prior to using our new fMRI analysis method. First, we perform a literature survey in order to determine which methods to compare against, and which manner of using context is preferable. Second, as in the object-detection case above, we define what performance criteria we use, both with respect to detection quality, and with respect to region sizes. Third, we create a dataset of simulated fMRI data to train and test our method. In Chapter 6, we present our new region-based method. We carry out experiments on the dataset mentioned above, and we compare the 
results to a standard method using the performance criteria mentioned above. This comparison procedure allows us to answer research question RQ3.

In order to answer research question RQ4, we use the performance criteria and dataset as mentioned above. However, we cannot compare our scale-estimation results to an existing method, since no currently existing method yields scale estimates. Therefore, we compare our scale-estimation results to the actual region scales used in creating the dataset. To test the scale-estimation performance over a range of scales, we use data with two different brain activation patterns, in which the actual scales of the specific regions differ.

For the answer of research question RQ5, we use a dataset obtained in a real fMRI session involving a test subject. We then define the criteria on which we select a dataset. Again, we compare our results to those of a standard method. However, in real fMRI data, the performance with respect to region detection and scale estimation cannot be measured in a quantitative manner. Therefore, we introduce qualitative performance criteria that are based on existing neurophysiological knowledge on the dataset at hand.

In order to answer research question RQ6, we need a dataset obtained in a real fMRI experiment involving a group of test subjects. In We present the selection criteria involved. Using the same qualitative performance criteria, we assess our method's performance. We compare the results to those of a single test subject to assess to what degree our method is robust to inter-personal differences.

In summary, our methodology consists of the following research approaches: machine learning, literature review, the formulation of definitions, the development of criteria, the creation of datasets, analysing a (new) method, comparing two methods, performing experiments, performing a variety of tests, and studying interpersonal differences.

\subsection{Thesis outline}

The thesis starts with an introduction (Chapter 1) on the use of context in image analysis. Then we formulate a general problem statement on the use of information from a local spatial context. The ideas on using context in image analysis are applied in two fully different application areas, viz. the cultural-heritage domain (Part I) and the medical domain (Part II).

Part I consists of four chapters and focusses on object detection. In Chapter 2 the domain is scrutinised, relevant concepts are introduced, and performance criteria are established. Finally, binary-classifier structures are discussed. Chapter 3 describes the details of the context-based object detection. An experimental methodology is given, experiments are executed, and results are discussed. The chapter summary provides adequate insight into the progress made. Chapter 4 describes a second approach of the context-based object detection, based on insights obtained in Chapter 3, viz. by using gradients. Many items are discussed and the results on a dataset of faces are analysed in detail. Chapter 5 provides a general discussion of object detection. It discusses the speed versus accuracy trade-off. Related work is discussed and future directions of research are given. 
In Part II (Chapters 6 to 8), we investigate the use of context in fMRI brain mapping. Chapter 6 introduces the fMRI brain-mapping problem, and the regionbased method, i.e., our context-based fMRI brain-mapping method. In addition, the Chapter show the results of applying the region-based method to simulated fMRI data. In Chapter 7, we apply the region-based method to real fMRI data, in a setting with multiple test subjects. In Chapter 8, we discuss the regionbased method in light of the results obtained in the Chapters 6 and 7 . We draw conclusions on the use of context in fMRI brain mapping.

Chapter 9 combines the results of Part I and Part II and aims at drawing general conclusions on the use of context in image analysis. Moreover, it gives directions for future research. 


\section{Part I}

\section{Object Detection}





\section{Chapter 2}

\section{Objects and their contexts}

Our research on the use of contextual information consists of two parts that are characterised by their domain of application. In this part we apply contextual information to the cultural-heritage application domain. The part consists of four chapters. In this chapter, we introduce the problem statement in the culturalheritage domain, and we introduce the datasets and performance criteria used in order to assess the context-based methods developed in our research. In the Chapters 3 and 4, we describe and assess two context-based methods particularly developed for the cultural-heritage domain: the context-detection method and the gradient method respectively. In Chapter 5, we discuss issues common to both methods, we place our methods in a broader perspective, and we draw conclusions.

\subsection{The cultural-heritage problem statement}

In the last decade, the automatic detection of objects in the cultural heritage has received a large amount of attention. Object detection by a computer is the automatic determination of the locations and sizes of objects in an image, where the objects belong to a predefined class. Object detection is an active research area that has yielded many methods (see, e.g., Hjelmås and Low (2001), Papageorgiou and Poggio (2000), Scheinderman and Kanade (2000), Sung and Poggio (1998), and Viola and Jones (2001a)). Current object-detection approaches convert the problem of object detection into a binary pattern-classification problem by defining regions containing the object to-be-detected as positive instances, and all other image regions as negative instances.

The problem of object detection is different from the problem of object localisation. In object detection, one does not know in advance how many (if any) instances from the object class of interest are present in a given image. In object localisation, one disposes of the additional information that exactly one instance is to be found in a given image. As object detection lacks this additional information, it is a harder problem to solve computationally.

As in any classification problem, the number of misclassifications should be minimised in object detection. Two types of misclassifications exist, viz. (1) the 
classifier detects an object where there is no object, and (2) the classifier detects no object where there is an object. More formally, starting from the null hypothesis that an image patch is not an object instance (i.e., it is a background pattern), the two types of misclassification are formally defined as follows. If a classifier incorrectly rejects the null hypothesis, i.e., it returns that an object is present in a region where, in reality, no object is present, the detection is called a false detection (in the literature, this is also referred to as a false positive, or type-I error). If a classifier fails to reject the null hypothesis, even though an object instance is present, the classification is called a missed detection (in the literature, this is also referred to as a false negative, or type II error). In the sequel, we will use the terms "false detection" and "missed detection".

Current research on automatic object detection has led to a variety of approaches that yield reasonable detection rates on natural images (Sung and Poggio, 1998; Papageorgiou and Poggio, 2000; Schneiderman and Kanade, 2000; Hjelmås and Low, 2001; Viola and Jones, 2001a). The main limitation of these approaches is that the detection rates are accompanied by high false detection rates. Our approach, to be detailed below, aims in particular at reducing the false-detection rate at any given detection rate.

Two concepts from behavioural sciences, spatial context and visual attention (Postma, 1994; Postma, Van den Herik, and Hudson, 1997), inspired us in mitigating the problem of false detections The first concept, spatial context, is important because observers use spatial context when determining the locations and sizes of the objects. Objects are found faster and more reliably if they are embedded in familiar contexts (Biederman, 1981), since such familiarity may prevent false detections of object-like patterns in unlikely contexts. Figure 2.1 shows two examples of false detections. In the figure, the small square images (left) are enlarged versions of the square regions indicated by boxes in the large images. Considered in isolation, both small images are highly similar to faces. When considered in their natural context, a potential interpretation as faces is quickly suppressed. It should be noted that the spatial context of the object contains both the object itself, and its direct spatial surroundings.

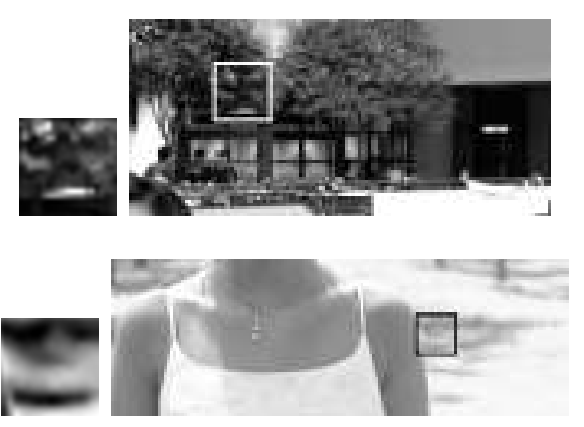

Figure 2.1: Examples of patterns that are similar to faces, but that are clearly not faces when viewed in their context. 
The second concept, visual attention, enables primates to select rather efficiently part of a visual scene (Lewis and Edmonds, 2003). This selection allows primates to reduce the amount of information to be processed, and to confine their attention to appropriate regions. Visual attention can be shifted overtly through eye movements (saccades) (Yarbus, 1967), or covertly by a mental process (Posner and Peterson, 1990). Overt attention focusses the appropriate region on the fovea, the high-resolution part of the retina (Palmer, 1999). Covert attention is a mental process that can be likened to an internal "spotlight" that is focussed on parts of the retina to select future locations for overt attention (Eriksen and Yeh, 1985; Eriksen and James, 1986; Humphreys and Bruce, 1989; Postma et al., 1997).

According to theories of human vision (Koch and Ullman, 1985), the concepts of spatial context and visual attention are closely related. Recent results in human perception studies (Lewis and Edmonds, 2003) suggest that visual attention in humans involves local contextual information as well as object features to direct the focus of attention to the object of interest.

In the Chapters 3 and 4, we focus on the following problem statement:

Problem statement: To what extent can the local spatial context of an object enhance the reliability of object detection in natural and painted images?

This problem statement is considered by posing the two research questions introduced in Chapter 1:

Research question 1 (RQ1): How can local spatial context be used to lower the number of false detections (i.e., achieve a more accurate detection) at an acceptable speed in automatic object detection?

Research question 2 (RQ2): What size of the context region is best suited to lower the false-detection rate?

The remainder of this chapter is outlined as follows. In Section 2.2, we describe our general approach in using context. Section 2.3 describes the datasets we use, and Section 2.4 provides the evaluation criteria for the object-detection performance of our methods. Finally, in Section 2.5, we introduce the binary classifiers that are used in both context-based object-detection methods.

\subsection{The use of context for object detection}

The use of context should facilitate object detection. Based on this straightforward idea we have designed a method consisting of two stages: a context-detection stage and an object-detection stage. The context-detection stage is concerned with the determination of appropriate contexts. The object-detection stage is responsible for the detection of objects. In this section we discuss the combination of both stages (2.2.1) and the definition of context (2.2.2). 


\subsubsection{The combination}

The two stages can be combined in two different ways, leading to two different realisations of context-facilitated object detection. In the first realisation, the context-detection stage precedes the object detection stage to limit the regions to be examined by the object classifier. In this realisation, the context-detector is used for selection. In the second realisation, the context-detection stage succeeds the object-detection stage in order to validate the spatial contexts of the detected objects. In this realisation, the context-detector is employed for validation. Both realisations differ in their implementation and may differ in computational efficiency. However, as both realisations effectively combine an object probability based on context and object information, they do not lead to differences in falsedetection rates. Therefore, in our methods we choose the realisation that is most efficient in terms of computation speed.

\subsubsection{The definition}

The appropriate definition of "context" is pivotal in our approach. We define context as a square spatial region centred around the object of interest. This leaves the size of the context to be determined. Using too small a region does not provide any more information than is already available to the object classifier, and thus cannot improve object-detection performance. Using too large a region will likely include too much non-descriptive data, and will thus also not improve objectdetection performance. The optimal context size will be determined empirically.

\subsection{Datasets in human-face detection}

We choose to test our methods on a single object class: the class of frontal human faces. Face detection is an appropriate challenge because it is well documented and data for comparison is readily available (Hjelmås and Low, 2001). For the task of frontal face detection, our definition of context implies that mainly features from both the face and the head form contextual cues.

In order to be able to validate the methods developed in our research, a labelled dataset needs to be defined. The labels are required (1) to train models, and (2) to validate the results obtained by applying the models. In this regard, a label consists of the coordinates of a hand-marked box around an actual face in a digital image.

We use two datasets. The first set is a natural-image set, and the second set is a portrait set.

\subsubsection{Natural-image set}

The natural-image set is obtained from the Internet, and consists of 995 images that together contain 3,383 frontal human faces. The images contain humans in a normal natural, urban, or indoor environment. The images contain a large 
number of background patterns that might be easily mistaken for faces, and thus represent a challenging dataset for our methods.

The natural-image set is split into two subsets: an evaluation set, called webset, and a training set. The web-set consists of 775 images from the natural-image set that together contain 1,885 labelled faces. The labelled faces are at least $30 \times 30$ pixels in size.

The training set consists of the 220 remaining images of the natural-image set. This set contains 1, 498 frontal human faces.

\subsubsection{Portrait set}

The portrait set is a subset of the AR faces database (Martinez and Benavente, 1998). The AR faces dataset consists of colour portrait photos against a white background. Each photo contains a single frontal human face. The set contains photos of 76 men and 60 women, each under 26 different variations of lighting, partial occlusion (i.e., sunglasses, scarfs), and facial expression (e.g., smiling, angry).

From these 26 combinations, we use the 14 combinations that correspond to unoccluded faces. The resulting set of $(76+60) \times 14=1,904$ images is referred to as the "portrait set".

\subsection{Performance criteria}

To assess the object-detection quality, one needs a consistent definition of the following three criteria: (1) the relative number of true detections, (2) the relative number of false detections, and (3) the relative number of missed detections (Smeulders et al., 2000). As there is no consensus over the exact definition of "true detection", "false detection" and "missed detection" in the object-detection field, we specify our definitions below.

In general, a face gives rise to multiple overlapping detections in close proximity. For the purpose of evaluation we employ a grouping procedure similar to Pham, Worring, and Smeulders (2002). The grouping procedure groups all regions classified as final detections with an area overlap of at least 90 per cent into a single detection. Each group is then considered a single detection, of which the coordinates are the means of the coordinates of the group members. From all detections, at least one should overlap sufficiently with a labelled face in order for that face to be a true detection; the scale of the detection should be within a factor 1.5 of the scale of the labelled face, and the detection's centre should be within a distance to the labelled face's centre no larger than 30 per cent of that face's size. If none of the detected windows satisfies these criteria for a given labelled face, that labelled face is a missed detection. Detections that do not satisfy the overlap criteria for any of the labelled faces are regarded as false detections.

Figure 2.2 shows an example of the grouping and status of the detections. The top panel of Figure 2.2 shows raw detections obtained using an object detector on an example image. Each box represents a detection. The centre panel of Figure 2.2 shows the results after grouping the detections. Finally, the bottom 

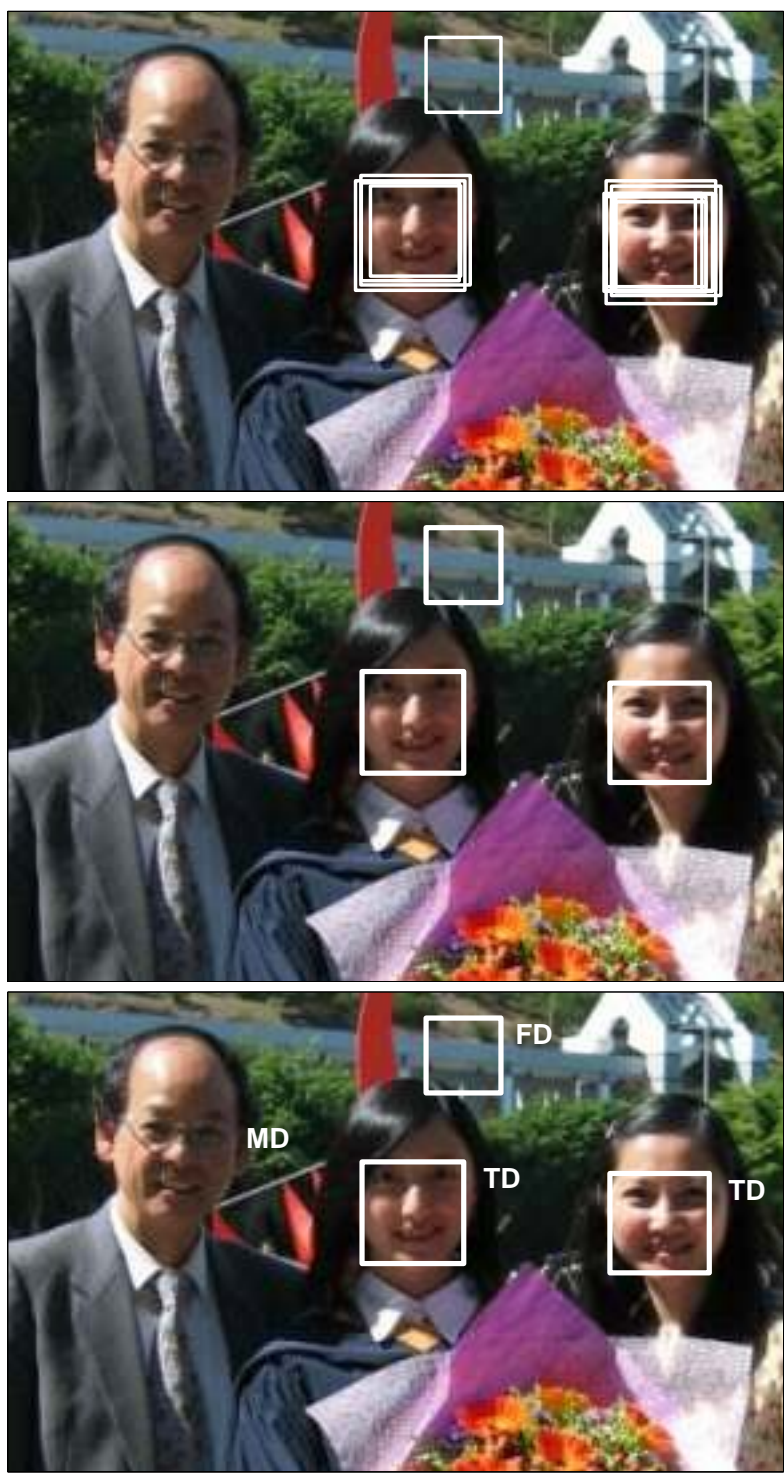

Figure 2.2: Detection grouping example. Top: ungrouped detections, centre: grouped detections, bottom: detection-group status.

panel of Figure 2.2 shows the result; it is clear that the rightmost two faces are detected (true detections), whereas the leftmost face is missed (missed detection). The detection group in the top centre of the image does not correspond to any of the faces; it is a false detection. 


\subsection{Binary-classifier structures}

As mentioned in Section 2.1, we treat object detection as a binary patternclassification problem. As such, we employ binary classifiers in both our contextbased object-detection methods. Below, we describe the two types of binary classifiers we use, viz., the monolithic classifier (2.5.1) and the boosted-cascade classifier $(2.5 .2)$.

\subsubsection{The monolithic classifier}

First, we employ a monolithic support vector machine (SVM) to detect objects. Despite the difficulties in training monolithic classifiers that have a sufficiently low false-detection rate, SVMs perform well and can be efficiently trained on moderately large datasets (Schölkopf and Smola, 2001). In addition, efficient software is readily available (Cawley, 2000; Rifkin, Nadermann, and Moreno, 2001). A quadratic kernel is employed, as results by Papageorgiou and Poggio (2000) indicate that such a kernel yields a good object-detection performance. Papageorgiou and Poggio (2000) use features from an overcomplete Haar-wavelet decomposition on two wavelet scales.

\subsubsection{The boosted-cascade classifier}

Second, we use the very efficient classifier framework developed by Viola and Jones (2001b). The main idea behind Viola and Jones' method is that it is rather difficult to build a monolithic classifier that combines a high detection rate with a very low false-detection rate $\left(\sim 10^{-6}\right.$ to $\left.10^{-7}\right)$ required in object detection. However, it is not so hard to build a weak classifier with a very high detection rate, if one allows that classifier to have a high false-detection rate; such a classifier is called a simple classifier. Figure 2.3 shows how multiple simple classifiers can be combined in a boosted cascade. The boosted cascade consists of $n$ (typically 20 to 25) stages, in which each stage is a simple classifier. If a given sample is classified as a negative by any stage, the classification is stopped immediately. If a sample is classified as a positive, it is fed to the next stage. If, and only if, a sample is classified positively by all stages, the corresponding image patch it is regarded as an object pattern. A typical 20-stage object classifier, in which each stage has a detection rate of, for instance, 0.995 and a false-detection rate of 0.5 , can achieve a detection rate of $0.995^{20}=0.90$ and a false-detection rate of $0.5^{20}=9.5 \cdot 10^{-7}$.

The above strategy for obtaining a high detection rate combined with a low false-detection rate can only function if an appropriate boosting method is used. Viola and Jones (2001b) use gentle AdABoost (Freund and Shapire, 1996) locally to train each of the simple classifiers. The simple classifier $i(i>1)$ is trained on all positive training samples classified as true detections by the stages 1 to $i-1$, and a fixed number of negative samples classified as false detections by the stages 1 to $i-1$.

The approach by Viola and Jones (2001b) has two advantages over monolithic classifiers. The first advantage is classification speed. This advantage is 


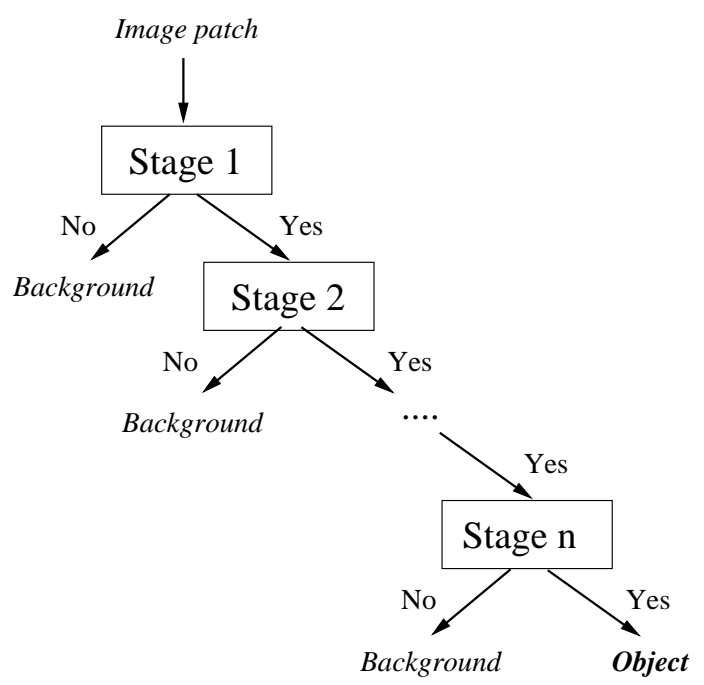

Figure 2.3: Overview of a boosted-cascade classifier

caused by the simplicity of the classifiers, combined with the fact that classification of an image patch is stopped once it is classified as negative by any stage. It implies that the vast majority of image patches can be rejected as background patterns after passing through only one, or a few, stages. The second advantage is the generalising performance; each classifier stage is trained on only positive and negative examples that were classified as positive by all previous stages. As the false-detection rate of all previous stages soon becomes quite small, the last stages are effectively trained on billions of negative samples. This improves the generalisation performance considerably. In contrast, it is not technically feasible to train one monolithic classifier on billions of samples.

Each classifier stage utilises Haar-wavelet features, which can be extracted in a very fast manner from images using the integral image framework developed by Viola and Jones (2001b), and extended by Lienhart, Liang, and Kuranov (2002). Lienhart et al. incorporated the classification method into the publicly-available Intel Open Computer Vision Library ${ }^{1}$.

${ }^{1}$ http://www. sourceforge.net 


\section{Chapter 3}

\section{Context-based object detection}

This chapter describes the work performed on the context-detection method. The chapter is partly based on the following publications ${ }^{1}$ :

1. Bergboer, N. H., Postma, E. O., and Herik, H. J. van den (2003). ContextEnhanced Object Detection in Natural Images. Proceedings of the BNAIC 2003 (eds. T. Heskes, P. Lucas, L. Vuurpijl, and W. Wiegerinck), pp. 27-34, Nijmegen, The Netherlands.

2. Bergboer, N. H., Postma, E. O., and Herik, H. J. van den (2006a). Accuracy versus Speed in Context-Based Object Detection. Pattern Recognition Letters, Vol. 28, No. 5, pp. 686-694. ISSN 0167-8655.

3. Bergboer, N. H., Postma, E. O., and Herik, H. J. van den (2006b). ContextBased Object Detection in Still Images. Image and Vision Computing, Vol. 24, No. 9, pp. 987-1000. ISSN 0262-8856.

\subsection{The context-detection method}

The context-detection method is motivated by the coarse-to-fine attention mechanism present in primates (Lewis and Edmonds, 2003). The coarse-to-fine attention mechanism works as follows. An image is initially analysed coarsely to identify promising areas (i.e., image regions likely to contain objects) and subsequently scrutinously examined in the selected regions. The context-detection method operates analogously, also in two stages (context detection and object detection). First, in the context-detection stage, the visual image is analysed in a rough-andready way on a course spatial resolution in order to find possible regions of interest.

\footnotetext{
${ }^{1}$ The author would like to thank his co-authors and the publishers of the BNAIC 2003 proceedings, Pattern Recognition Letters, and Image and Vision Computing, for their kind permission to reuse relevant parts of the articles in this thesis.
} 
Second, in the object-detection stage, a computationally more expensive analysis on a fine spatial resolution is performed in the regions of interest. In terms of the two realisations discussed in Section 2.2, the context-detection method adopts the selection realisation, because the context-detection stage precedes the objectdetection stage.

The two-stage strategy can be implemented in various ways. We choose a window-sliding technique in both stages, viz. for the selection of context regions and for the selection of object regions. The reasons are: (1) computational efficiency and (2) the fact that this technique is widely used in visual detection methods, e.g., Sung and Poggio (1998), Schneiderman and Kanade (2000), and Papageorgiou and Poggio (2000). In window-sliding techniques, a square window of a given size is slid over the image at different scales. At each position and scale, the contents of the window is used for deciding if it represents a likely context or object. In the Sections 3.2 and 3.3 we discuss both detection stages in more detail.

\subsection{Context detection}

In the context-detection stage an image is searched at a coarse scale for likely spatial contexts of the object class of interest. The stage is illustrated in the left panel of Figure 3.1. The bold square represents the search window at a given location. The other squares represent detected contexts.

We define an object's spatial context as a square region of the image, of which the lateral dimensions are larger than those of the square region enclosing the object. The visual features within this confined spatial region around a centred object include features that are both internal and external to the object

The context-detection stage results in the selection of a number of context regions.
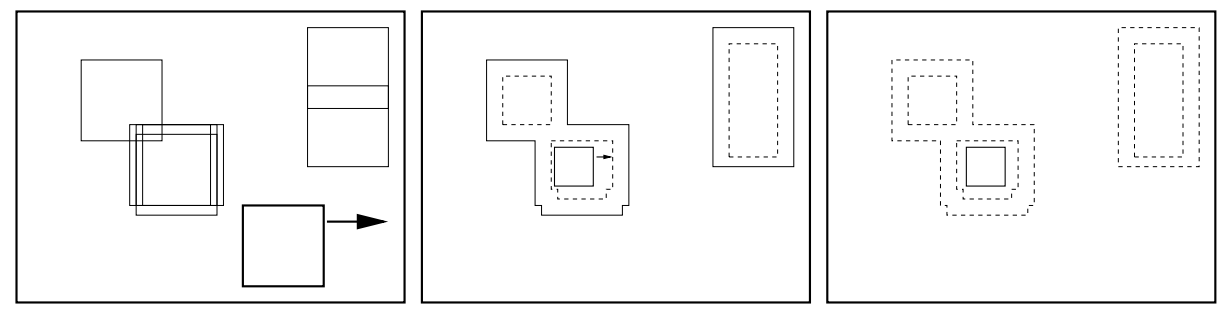

Figure 3.1: Graphical representation of the context-detection method at a given scale.

As we do not know a priori which context size is optimal for our method, we investigate three context sizes. The first context size is named "PPcon", as it comprises a region twice the lateral size of a face label as defined by Papageorgiou and Poggio (2000). The second context size is named "VJcon", as it comprises a region twice the lateral size of a face label as defined by Viola and Jones (2001b). The third context size is named "VJ2con", as it comprises a region that is laterally 


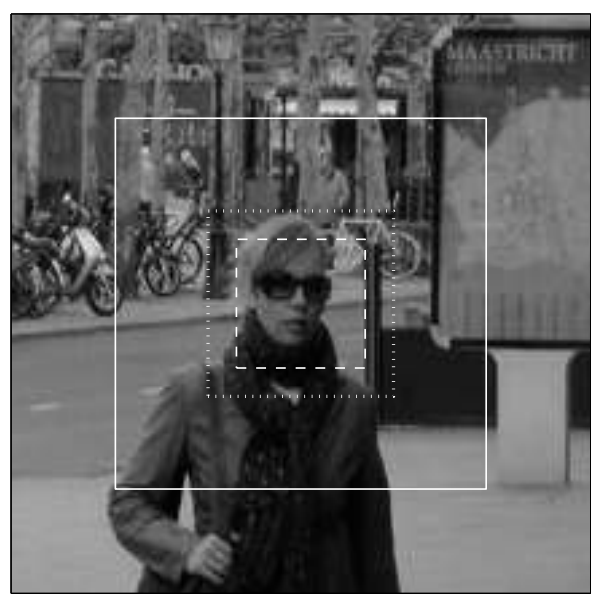

Figure 3.2: The three different context types used in the context-detection method. Dashed: PPcon. Dotted: VJcon. Solid: VJ2con.

twice as large as the "VJcon" region. The three context sizes are illustrated in Figure 3.2. As we normalise all face detections to the size defined by Papageorgiou and Poggio ${ }^{2}$, the PPcon, VJcon, and VJ2con context sizes are 2, 2.8, and 5.6 times the lateral size of our face labels, respectively.

\subsection{Object detection}

In the object-detection stage, objects are searched for and detected at a fine scale within the centre parts of selected context regions from the first stage. The object-detection stage is illustrated in the centre panel of Figure 3.1. Here, the bold square with the array represents an object-detection window. The regions outlined by solid lines are the union of all detected contexts. The dashed regions represent the centre parts of the contexts that are searched by the object detector. The right panel of Figure 3.1 is the final state of the second stage; it shows the final detection result.

Within detected context regions, the object classifier examines all locationscale combinations that lie within a factor 1.5 of the expected scale of a face. The expected scale depends on the type of the context window. For instance, for the VJcon context, a face is expected to be the half of the context size.

\subsection{Experimental set-up}

To assess the generalisation performance of the context-detection method, the three context classifiers - one for each of the context-types shown in Figure 3.2 -

\footnotetext{
${ }^{2} \mathrm{~A}$ square box that extends from just above the brow to just below the chin.
} 
are trained on the contexts of the 1,421 faces in the training set. In the contextdetection stage, we aim to use a boosted-cascade classifier with 20 classifier stages. A window size of $20 \times 20$ pixels is used, and 3,000 negative examples are used in the training of each of the 20 classifier stages. Each of the stages is trained to have a detection rate of 0.995 and a false-detection rate of 0.5 . This would theoretically yield a classifier with a detection rate of $0.995^{20}=0.905$ and a false-detection rate of $0.5^{20}=9.5 \cdot 10^{-7}$ (cf. 2.5.2).

In the object-detection stage, we use the standard 25-stage Viola-Jones frontal face-detector (Viola and Jones, 2001b), which has been extended (Lienhart et al., 2002) and which is now part of the Intel Open Computer Vision library ${ }^{3}$. In order to assess the benefit of using contextual information in our method, we compare its results to those obtained with the object detector alone. We will refer to the latter method as the brute-force method.

To ensure that all faces are found, the images are classified for face sizes of $24.5 \times 24.5$ pixels and up. To this end, a scale-space pyramid of the image is calculated in which subsequent scales differ by a factor 1.1 .

\subsection{Results}

The experiments outlined in the previous section have been carried out on a 2.4 $\mathrm{GHz}$ Pentium 4 desktop computer running GNU/Linux ${ }^{4}$. We will first present the results on object-detection accuracy, and then present data on calculation times at a later stage.

Figure 3.3 shows the object-detection accuracy results for the three contextdetection methods (PPcon, VJcon, and VJ2con) and the brute-force method. The figure is a Receiver Operating Characteristic (ROC) curve that shows the tradeoff between the detection rate (vertical axis) and the number of false detections per image (horizontal axis). It is obtained by recording the detection rate and false-detection rate of all methods at different thresholds above which a classifier output is considered to be a detection. The curve for the brute-force detector is obtained by varying the threshold on the output of the object detector. The curves for the PPcon, VJcon, and VJ2con methods are obtained by varying the threshold on the output of the context detector; the object detector is left in its original configuration. Table 3.1 provides numerical false-detection rates at a number of given points in the ROC curves; at detection rates that are useful in a practical setting: $80 \%, 85 \%$, and 90\%. For example, the PPcon method yields 0.255 false detections per image on average over all images on the test set, at a detection rate of $80 \%$. This means, it yields slightly more than one false detection in every four images, while detecting $80 \%$ of all labelled faces. Consistent with our research question for this chapter, a lower false-detection rate corresponds to a better (i.e., more accurate) method.

From these results, we make two observations. The first observation concerns the detection performance. It is clear from the results that the detection perfor-

\footnotetext{
${ }^{3}$ http: //www . sourceforge.net

${ }^{4}$ http://www.debian.org
} 
Table 3.1: False detections per image at several detection rates, for the three contextdetection methods.

\begin{tabular}{l|l|l|l}
\multirow{2}{*}{$\begin{array}{l}\text { Context } \\
\text { Type }\end{array}$} & \multicolumn{3}{|c}{ FD rates at detection rate } \\
\cline { 2 - 4 } & $80 \%$ & $85 \%$ & $90 \%$ \\
\hline PPcon & 0.255 & 0.374 & 1.159 \\
VJcon & 0.267 & 0.475 & 1.314 \\
VJ2con & 1.001 & 1.279 & 1.535 \\
\hline Brute-force & 0.296 & 0.465 & 1.135 \\
\hline
\end{tabular}

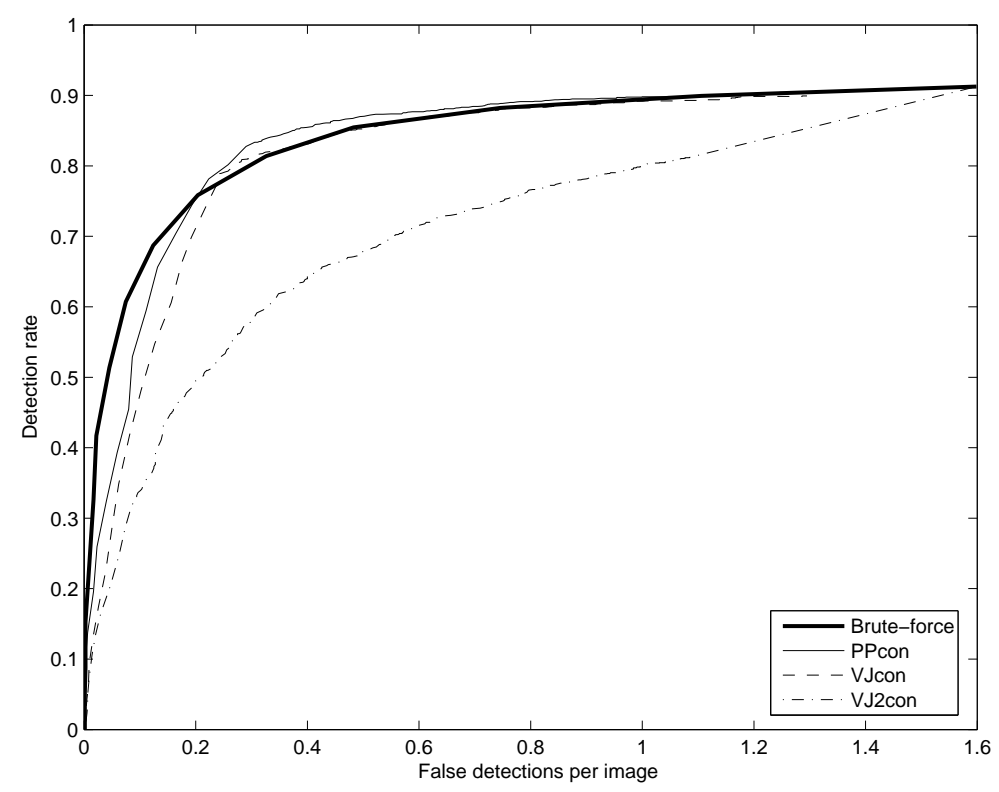

Figure 3.3: Accuracies obtained with three context-detection methods and a brute-force object classifier.

mance of the PPcon and VJcon methods is comparable to that of the brute-force object detector. For detection rates above $80 \%$, the PPcon method slightly outperforms the brute-force object classifier. For detection rates below 80\%, both the PPcon and VJcon methods perform worse than the brute-force object detector. The VJ2con method performs significantly worse than the brute-force object detector for all detection rates. In terms of detection accuracy, we thus observe that a context size that is 2 (PPcon) to 2.8 (VJcon) times the size of a face label, is the best choice.

The second observation concerns the computational time required to obtain the detection results. Table 3.2 shows timing results for the three context-detection methods, for three well-known image resolutions: $800 \times 600$ pixels (SVGA), $640 \times$ 480 pixels $(\mathrm{VGA})$, and $320 \times 240$ pixels $(\mathrm{QVGA})$. For each image resolution, the table shows the brute-force time, i.e., the time the brute-force object detector 
requires to analyse the image. In addition, the table contains the time required by the combined context-detection method: the time for the context stage, the time for the subsequent object-detection stage, and the sum of both timings. Finally, in the last column, the speed-improvement factor, i.e., the factor by which the context-detection method is faster than the brute-force object detector, is listed.

\begin{tabular}{l|r|l|r|r|r|r}
\hline \multicolumn{6}{c}{ Table 3.2: Comparison of times required for detection, for different image sizes. } \\
\hline $\begin{array}{l}\text { Image size } \\
(\text { pixels })\end{array}$ & $\begin{array}{r}\text { Brute-force } \\
\text { time }(\mathrm{ms})\end{array}$ & $\begin{array}{l}\text { Context } \\
\text { type }\end{array}$ & $\begin{array}{r}\text { Context } \\
\text { time }(\mathrm{ms})\end{array}$ & $\begin{array}{r}\text { Object } \\
\text { time }(\mathrm{ms})\end{array}$ & $\begin{array}{r}\text { Total } \\
\text { time }(\mathrm{ms})\end{array}$ & $\begin{array}{r}\text { Speedup } \\
\text { factor }\end{array}$ \\
\hline $800 \times 600$ & 1,678 & PPcon & 268 & 180 & 448 & 3.74 \\
& & VJcon & 340 & 188 & 529 & 3.11 \\
& & VJ2con & 558 & 145 & 702 & 2.31 \\
\hline $640 \times 480$ & 1,124 & PPcon & 164 & 161 & 325 & 3.46 \\
& & VJcon & 218 & 169 & 387 & 2.90 \\
& & VJ2con & 356 & 138 & 494 & 2.33 \\
\hline $320 \times 240$ & \multirow{2}{*}{259} & PPcon & 30.3 & 62.2 & 92.5 & 2.80 \\
& & VJcon & 46.3 & 68.8 & 115 & 2.24 \\
& & VJ2con & 67.5 & 54.9 & 122 & 2.31 \\
\hline \hline
\end{tabular}

The above two observations show that there exists a trade-off between detection accuracy and detection speed; the context-detection method yields a slightly less accurate object detection than the brute-force method, but does run faster than the brute-force method.

It is clear from the results that the largest speed increase is obtained when using the PPcon method. For the VJcon and VJ2con methods, the speed increase becomes less, although both are still faster than the brute-force object detector.

\subsection{Discussion}

Our results obtained with the three context-detection methods give rise to two considerations in relation to the cultural-heritage problem statement. Below, we discuss both considerations.

The first consideration is about the global pattern of accuracy and speed performances obtained with the method (3.6.1). The second consideration is related to the observations on the effect of window size (3.6.2). Finally, related work is discussed (3.6.3).

\subsubsection{General considerations on accuracy and speed}

We observed that the reduction of false detections is small to nonexistent in the context-detection method. We assume that this is caused by a combination of two factors. The first factor is that the intra-class variance of facial contexts is larger than that of the faces, because the facial context includes parts of the (varying) scene behind the person's head. Second, the possible number of features is large. The above combination is known as the Curse of Dimensionality (Bellman, 1972), and represents a hard problem for machine-learning algorithms in general, 
and face detection in particular. If domain knowledge can be integrated in the learning algorithm, the Curse of Dimensionality could possibly be mitigated, as the knowledge can be used to tailor the learning problem. As mentioned in Section 2.1, Biederman (1981) showed that objects are found faster and more reliably when they are embedded in familiar contexts, because humans use the spatial relations between parts of the context to locate an object. Thus, certain parts of the context might convey more information (or, have a lower intra-class variance) than others. So, we expect that including domain knowledge on the spatial relations between context parts and the object, might make the use of context in object detection a tractable problem. The inclusion of domain knowledge implies that we have to choose a specialised classifier design. This problem will be discussed in-depth in the next chapter.

Although the number of false detections was hardly reduced, a considerable increase in speed was achieved, viz. a speed increase by a factor of 3.74 was achieved on images of size $800 \times 600$ pixels. As noted in Section 2.2, the order of the context-detection stage and the object-detection stage is a method of implementation choice. In case of the context-detection method, the context-detection stage apparently (1) is fast enough, and (2) reduced the search space sufficiently, that using it as a first stage improves the detection speed. The reverse order is theoretically equivalent (one would check whether object candidates fall within detected contexts), but would be slower.

\subsubsection{Small windows versus large windows}

We observed that the use of smaller context windows yields a better detection accuracy than the use of larger context windows. The difference is most likely due to the larger intra-class variance for larger windows. This phenomenon will be discussed in more detail in Section 5.2.

Regarding the detection speed, it is expected that the use of small windows would worsen performance; for small windows, the image has to be analysed down to smaller scales, which involves a larger number of model evaluations. However, the context-detection methods using small windows are faster than those using large windows, despite the larger number of model evaluations. The reason for this seems to be the larger intra-class variance for larger windows. A large intra-class variance increases the amount of features in the detection cascade, which slows down the detection speed. Apparently, the advantage of having a less complex context classifier outweighs the disadvantage of having to search down to smaller scales.

\subsubsection{Related work}

Kruppa et al. (2003) determine face locations using a coarse detection stage similar to our first stage. However, they only detect the local context, and then assume that this context contains a face, rather than combining their method with an actual face detector. Their approach yields good results in situations with low- 
complexity backgrounds, such as video-conferencing scenes. In some respect, the context-detection method can be regarded as an extension of their method.

\subsection{Chapter summary}

This chapter discussed the context-detection method, in which we used the coarseto-fine mechanism of attention present in primates (Lewis and Edmonds, 2003).

The results show a clear trade off between detection speed and detection accuracy. The use of context detection for object detection yields a reduction of the search space for the object detector, and thus allows for faster object detection. However, the increased speed comes at the price of a slight reduction in detection accuracy. The results show that there is an optimal size for the region that is defined to be the object's context. In the case of face detection, this is a region with lateral dimensions that are a factor 2 to 2.8 larger than the face. Using larger regions leads to a large decrease in detection accuracy.

As such, even though the context-detection method brings about a considerable increase in detection speed, it does not solve our primary research goal of reducing the number of false detections. In the discussion above, we assume that this is due to the large intra-class variance of large regions, combined with the large number of possible features. 


\section{Chapter 4}

\section{Gradient-based context-based object detection}

In this chapter, our research on gradient methods will be discussed. This chapter is based on the following publications ${ }^{1}$ :

1. Bergboer, N. H., Postma, E. O., and Herik, H. J. van den (2004). A ContextBased Model of Attention. Proceedings of the 16th European Conference on Artificial Intelligence, ECAI 2004 (eds. R. López de Mánteras and L. Saitta), pp. 927-931, IOS Press, Valencia, Spain. ISSN 0922-6389.

2. Bergboer, N. H., Postma, E. O., and Herik, H. J. van den (2006a). Accuracy versus Speed in Context-Based Object Detection. Pattern Recognition Letters, Vol. 28, No. 5, pp. 686-694. ISSN 0167-8655.

3. Bergboer, N. H., Postma, E. O., and Herik, H. J. van den (2006b). ContextBased Object Detection in Still Images. Image and Vision Computing, Vol. 24, No. 9, pp. 987-1000. ISSN 0262-8856.

In Section 4.1, we introduce the manner in which we integrate domain knowledge on relative locations into our method. The integration results in the gradient method, that will be described in detail in Section 4.2. In Section 4.3, we describe the algorithmic approach used. In Section 4.4 we describe the experimental set-up, and in Section 4.5 we describe the associated evaluation procedure. We provide the experimental results in Section 4.6, and we discuss them in Section 4.7. Finally, we draw conclusions for the results of this chapter in Section 4.8.

\footnotetext{
${ }^{1}$ The author would like to thank his co-authors and the publishers of the ECAI 2004 proceedings, Pattern Recognition Letters, and Image and Vision Computing, for their kind permission to reuse relevant parts of the articles in this thesis.
} 


\subsection{Domain knowledge on relative locations}

In the previous chapter, we conjectured that if domain knowledge can be integrated in the learning algorithm, the Curse of Dimensionality could be mitigated. In particular, knowledge about the spatial relations between parts of the context and the object should somehow be incorporated in the detection method. In this chapter, a new method - that does exploit spatial relations - will be discussed: the gradient method.

We reiterate the problem statement from Chapter 2:

Problem statement: To what extent can the local spatial context of an object enhance the reliability of object detection in natural and painted images?

In this chapter, we will use domain knowledge on relative locations, which means that we will augment our cultural-heritage research questions with the following question:

Research question 1a (RQ1a): To what extent can knowledge of relative locations within the context help lower the false-detection rate?

In order to use knowledge on relative locations, we will employ machinelearning methods to classify and estimate the locations. This leads to a second question:

Research question 1b (RQ1b): What classifier design and feature sets are optimal for the gradient method?

\subsection{The gradient method}

The main problem encountered in the context-detection method is the high intraclass variance in the class of spatial contexts. When combined with the potentially very high-dimensional feature space, learning a well-generalising classifier becomes a difficult task. A possible solution to this problem could be found in the integration of domain knowledge to reduce the dimensionality of the learning problem.

Below, we take the domain knowledge into account by using small parts of the immediate visual surroundings, and attempting to focus attention in the spatial direction of the object. In order to detect objects, one needs to learn the relation between parts of an object's surroundings, and their location with respect to the object (the "gradient"). We try to employ this mechanism in the gradient method.

Our gradient method proceeds in two stages: (1) an object-detection stage followed by (2) a context-validation stage. So, in contrast to the context-detection method, the gradient method adopts the validation realisation of object detection (cf. 2.2.1) In the following two subsections we discuss both stages.

The dual-stage nature of the gradient method renders it independent of the type of object classifier. That is, arbitrary object classifiers can be used as "plugins" in the first stage. To emphasise the flexibility of the method, we shall employ 
the two different object classifiers introduced in Section 2.5: the monolithic classifier and the boosted-cascade classifier.

In both detection stages, the contents of a window is first transformed into visual features that are meaningful in terms of human vision (Palmer, 1999). The choice of features is essential for obtaining good results.

\subsubsection{The object-detection stage}

In the object-detection stage the image is scanned with an object detector using a window-sliding technique, as introduced in Section 3.1. This stage is illustrated in the left panel of Figure 4.1. The solid box with an arrow denotes the sliding window. The stage results in a set of object candidates, denoted by the other solid boxes in the left panel of Figure 4.1.

Experiments are performed with both object detectors introduced in Section 2.5; the associated visual features are described in Subsection 4.4.2.

\subsubsection{The context-validation stage}

In the context-validation stage, confidence is assigned to each of the first-stage object candidates. A sliding-window technique is used in locations close to the object candidates only. The contents of a window are used to obtain a probability density function (PDF) that describes the most probable relative location for the object with respect to the current location. For a given location, the PDF is illustrated in the centre panel of Figure 4.1. The solid square defines the region from which information is extracted and the arrow represents the expectation value of the relative location of the object with respect to the current location. The circles are contour lines for the probability density function. The probability density value at locations corresponding to first-stage object candidates (denoted by the dashed squares) is stored for each of these candidates.

The algorithm that generates the PDFs is trained on example patterns taken from the spatial context of the object class of interest. As noted in Section 3.2, we define the object as part of its spatial context; we can thus expect that features from the object itself and features from its surroundings will both be used to guide the visual attention process. The main difference between the gradient method and other methods based on object detection (Kruppa, Santana, and Schiele, 2003; Schneiderman and Kanade, 2004) is that the gradient method detects objects by combining local contextual cues into an estimate of the location of the object with respect to the current location.

In order to obtain a confidence value for each object candidate, PDFs for all locations that overlap an object detection are combined. The result of the second stage is a confidence value for each of the first-stage object candidates.

The final result of the gradient method is obtained by thresholding the confidence value, and regarding only those object candidates with a sufficient confidence value as final detections. These final detections are shown in the right panel of Figure 4.1. 

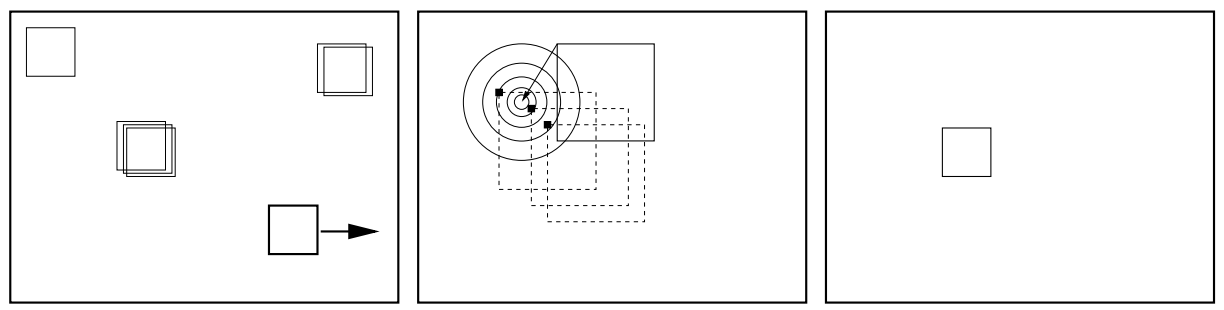

Figure 4.1: Graphical representation of the gradient method.

The visual features for the context-validation stage have to fulfil the following two requirements: (1) provide sufficient information about the spatial context of an object, and (2) they should give rise to a low intra-class variance for the context of the object. This means that the features should encode information that does not vary too much over the class of local spatial contexts of objects.

One of the earliest stages in human vision is the detection of edges at several orientations in the retinal image (Palmer, 1999). Consequently, many existing algorithms in the area of computer vision use features that are derived from edge information, such as wavelet features (Papageorgiou and Poggio, 2000) and scalespace features (Ter Haar Romeny and Florack, 2000).

We use overcomplete Haar-wavelet features (Papageorgiou and Poggio, 2000) for our context-validation stage. The reason for choosing Haar-wavelet features is that it is one of the most elementary features that encodes edge information at different orientations and that simultaneously can be calculated efficiently. The reason for choosing an overcomplete representation is twofold. First, it is better in capturing constraints between neighbouring regions and complex patterns than a complete representation (Papageorgiou and Poggio, 2000). Second, despite being overcomplete, it is sufficiently small in the number of features as compared to the Viola-Jones feature set. Since the number of training samples for contextual validation is small, a limited number of features is a prerequisite to prevent overfitting (see, e.g., Schölkopf and Smola (2001)).

The scales that contain the largest possible amount of the information unique to the object's context are included. These scales are specific to the object class of interest; we will elaborate on the scales used for the object class of faces, in Subsection 4.4.2.

\subsection{Algorithmic Approaches}

Below we describe the approaches used in the object-detection and the contextvalidation stages. Subsection 4.3.1 describes the two classifier structures used in the object-detection stage.

Since, the main focus of the context-validation stage is to learn the relation between patterns and their relative positioning within the direct spatial context of an object. Subsection 4.3.2 describes the relative localisation in more detail. 
We employ two different techniques to estimate PDFs: cluster-weighted models (Subsection 4.3.3), and decision trees (Subsection 4.3.4).

\subsubsection{Object classifiers}

We use the two different classifier structures introduced in Section 2.5 to detect objects. First, we employ a SVM introduced in Subsection 2.5.1. Second, we use the boosted-cascade classifier introduced in Subsection 2.5.2.

\subsubsection{Relative localisation}

Our objective is to estimate the location of the object relative to the current location, i.e., to estimate the vector $\overrightarrow{x_{r}}=\left(x_{r}, y_{r}\right)$ by means of the window features $v$, where $x_{r}$ represents the horizontal relative location and $y_{r}$ represents the vertical relative location. The function that minimises the mean square error between the estimated $\widehat{\overrightarrow{x_{r}}}$ and the real relative location $\overrightarrow{x_{r}}$ is the conditional expected value (Montgomery and Runger, 1994, p. 247):

$$
\widehat{\overrightarrow{x_{r}}}=\int \overrightarrow{x_{r}} f\left(\overrightarrow{x_{r}}, v\right) d \overrightarrow{x_{r}}
$$

where the joint PDF $f\left(\overrightarrow{x_{r}}, v\right)$ describes the relation between the two random variables $\overrightarrow{x_{r}}$ and $v$. It is given by:

$$
f\left(\overrightarrow{x_{r}} \mid v\right)=\frac{f\left(\overrightarrow{x_{r}}, v\right)}{f(v)} .
$$

As will be discussed in the next subsection, determining the relative object location $\overrightarrow{x_{r}}$ transforms into finding the joint $\operatorname{PDF} f\left(\overrightarrow{x_{r}}, v\right)$.

\subsubsection{Estimating the PDF using cluster-weighted models}

For building the relative location estimator, we need to estimate the joint PDF $f\left(\overrightarrow{x_{r}}, v\right)$. In the framework of regression algorithms, several approaches have been proposed for the estimation of joint PDFs. We will use cluster-weighted modelling (Gershenfeld, 1999), as it provides a straightforward algorithm for the learning stage (Torralba and Oliva, 2002; Verbeek, Vlassis, and Kröse, 2003). For completeness, we reproduce the main expressions of the estimation algorithm here.

In the cluster-weighted modelling algorithm, a joint PDF is expanded as a sum of $m$ elliptical Gaussian clusters that each model the local relationship between the $n$-dimensional input distributions and $\ell$-dimensional output distributions. In our specific application an output dimensionality of $\ell=2$ is used.

$$
f\left(\overrightarrow{x_{r}}, v\right)=\sum_{i=1}^{m} g\left(\overrightarrow{x_{r}} \mid v, c_{i}\right) g\left(v \mid c_{i}\right) p\left(c_{i}\right)
$$


in which $c_{i}$ refers to the $i$ th cluster. Moreover, $p\left(c_{i}\right)$ is a cluster weight, and $g\left(v \mid c_{i}\right)$ is a multivariate elliptical Gaussian with mean $\mu_{i}$ and covariance matrix $X_{i}$ defining the domain of influence in the input space of the cluster:

$$
g\left(v \mid c_{i}\right)=\frac{\exp \left[-\frac{1}{2}\left(v-\mu_{i}\right)^{T} X_{i}^{-1}\left(v-\mu_{i}\right)\right]}{(2 \pi)^{(n / 2)} \sqrt{\operatorname{det}\left(X_{i}\right)}} .
$$

The output distribution of the cluster is modelled by a similar expression:

$$
g\left(\overrightarrow{x_{r}} \mid v, c_{i}\right)=\frac{\exp \left[-\frac{1}{2}\left(\overrightarrow{x_{r}}-a_{i}-b_{i}^{T} v_{t}\right)^{T} S_{i}^{-1}\left(\overrightarrow{x_{r}}-a_{i}-b_{i}^{T} v_{t}\right)\right]}{(2 \pi)^{(\ell / 2)} \sqrt{\operatorname{det}\left(S_{i}\right)}} .
$$

The centres of the output clusters are dependent on the input samples $v_{t}$ by a linear transformation (described by the scalars $a_{i}$ and vectors $b_{i}$ ); their covariance matrices are $S_{i}$. Given the model parameters, the joint PDF for the output is given by:

$$
f\left(\overrightarrow{x_{r}} \mid v\right)=\frac{\sum_{i=1}^{m} g\left(\overrightarrow{x_{r}} \mid v, c_{i}\right) g\left(v \mid c_{i}\right) p\left(c_{i}\right)}{\sum_{i=1}^{m} g\left(v \mid c_{i}\right) p\left(c_{i}\right)}
$$

When estimating $\overrightarrow{x_{r}}$ given $v$, the expectation value $\widehat{\overrightarrow{x_{r}}}$ is calculated as

$$
\widehat{\overrightarrow{x_{r}}}=\frac{\sum_{i=1}^{m}\left(a_{i}+b_{i}^{T} v_{t}\right) g\left(v \mid c_{i}\right) p\left(c_{i}\right)}{\sum_{i=1}^{m} g\left(v \mid c_{i}\right) p\left(c_{i}\right)},
$$

and a covariance-matrix estimate, that functions as an inverse confidence measure, is calculated as

$$
\hat{S}_{\overrightarrow{x_{r}}}=E\left[\left(\widehat{\overrightarrow{x_{r}}}-\overrightarrow{x_{r}}\right)\left(\widehat{\overrightarrow{x_{r}}}-\overrightarrow{x_{r}}\right)^{T} \mid v\right]=\frac{\sum_{i=1}^{m} S_{i} g\left(v \mid c_{i}\right) p\left(c_{i}\right)}{\sum_{i=1}^{m} g\left(v \mid c_{i}\right) p\left(c_{i}\right)} .
$$

The estimated relative location $\widehat{\overrightarrow{x_{r}}}$ and the estimated covariance matrix $\hat{S}_{\overrightarrow{x_{r}}}$ are used to calculate a one-cluster PDF. The image-wide probability measure is formed by adding all the one-cluster PDFs.

\subsubsection{Estimating the PDF using decision trees}

In addition to the cluster-based model method discussed in the previous subsection, we use a C4.5 decision-tree method to estimate the joint PDF $f\left(\overrightarrow{x_{r}}, v\right)$. A C4.5 decision tree (Quinlan, 1993) maps its input attributes $v$ onto $m$ output classes. For each output class $c_{i}$, a conditional probability $p\left(c_{i} \mid v\right)$ is determined such that $\sum_{i=1}^{m} p\left(c_{i} \mid v\right)=1$.

In the gradient method, we employ a set-up in which the area covered by possible relative locations is discretised into a grid of $n$ rows and $n$ columns. In this way, using $m=n^{2}$, we may regard the conditional class probabilities yielded by the decision tree as a discretised relative location PDF. 


\subsection{Experimental set-up: human-face detection}

In this section, we describe the experimental set-up to validate our gradient method. Below, we describe the experimental procedure in three steps: the detection of face candidates (4.4.1), the contextual validation (4.4.2), and the training (4.4.3).

\subsubsection{Detection of face candidates}

For the detection of face candidates we use the monolithic detector and the boosted-cascade detector introduced in Subsection 2.5.

The monolithic detector is based on the method of Papageorgiou and Poggio (2000) with grey-scale wavelets, as these yield the most compact representation of the object class. A sliding window of size $19 \times 19$ pixels is used and the feature set consists of wavelet coefficients for square-support Haar wavelets of size $2 \times 2$ and $4 \times 4$. We use an overcomplete representation of $17 \times 17$ coefficients for a given scale and orientation. For both scales, the low-pass filtered data is discarded and the absolute value of only the horizontal, vertical, and diagonal detail coefficients are retained. Each of the resulting six components is normalised by its sum, thus a feature vector $\theta_{O} \in \mathbb{R}^{1,734}$ is obtained by vectorising and concatenating the six components.

In the boosted-cascade detector, the choice of line features and edge features used for classification is determined by the training algorithm.

\subsubsection{Contextual validation}

For the contextual validation we use a window of size $19 \times 19$ pixels. Contextvalidation models are trained on patterns from a region almost twice as large as the face itself. Each training face is down-scaled such that its dimensions are $19 \times 19$ pixels. The patterns used have a relative displacement from -8 to +8 pixels in both the horizontal and vertical direction.

Figure 4.2 illustrates the extent of the training region; the region is overlaid on a high-resolution face. The large dotted square denotes the region from which the training samples are taken, the dashed square in the centre denotes the actual face location, and the solid square in the upper left corner of the training region denotes the window for relative displacement of -8 pixels in both the horizontal and vertical direction in the down-sampled image ${ }^{2}$.

We use one of three different groups of overcomplete wavelet feature sets (Papageorgiou and Poggio, 2000) in order to determine which feature set best describes appropriate contexts of faces while incorporating the least amount of uninformative information.

\section{Coarse representation:}

\footnotetext{
${ }^{2}$ We show the training region overlaid on a high-resolution face in Figure 4.2 to illustrate clearly what portion of the head is used as context. When extracting features from a facial context, the high-resolution image is first down-scaled in such a way that the face has a size of $19 \times 19$ pixels.
} 


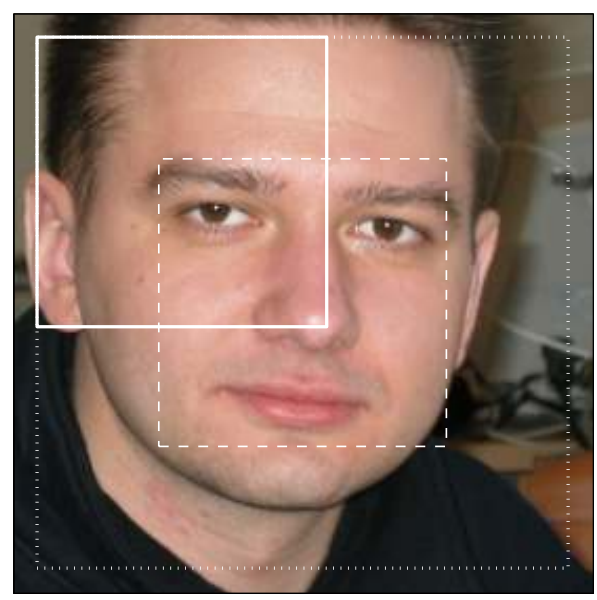

Figure 4.2: The region used for obtaining training samples, overlaid on a face.

- Coarse scale-2: Wavelets at decomposition level 1

- Coarse scale-4: Double-resolution wavelets at decomposition level 2

\section{Fine representation:}

- Fine scale-2: Double-resolution wavelets at decomposition level 1

- Fine scale-4: Quad-resolution wavelets at decomposition level 2

\section{Multiscale representation:}

- Double-resolution wavelets at decomposition level 1 (wavelet scale 2) and quad-resolution wavelets at decomposition level 2 (wavelet scale 4)

Figure 4.3 illustrates what contextual features are common to instances of the class of facial contexts; we show averages of the multiscale features over the entire training region. The top row shows double-res scale- 2 wavelets while the lower row shows quad-res scale- 4 wavelets. The three columns show horizontal details, vertical details, and diagonal details, respectively. The features actually used in the context-validation stage are windows of size $19 \times 19$ cropped from the training region, as indicated in Figure 4.3. The figure clearly shows that the multiscale representation captures dominant large features such as the eyes, the mouth, and the edge of the head. In particular, the internal context (eyes, nose, mouth) appears to be more important than the external context (edge of the head and background). In addition, it should be remarked that the differences between scale- 2 and scale- 4 wavelets is rather small, which suggests that the useful information resides in actual physical parts of the object or its surroundings. This conforms to contemporary studies, such as described in Vinette, Gosselin, and Schyns (2004). 

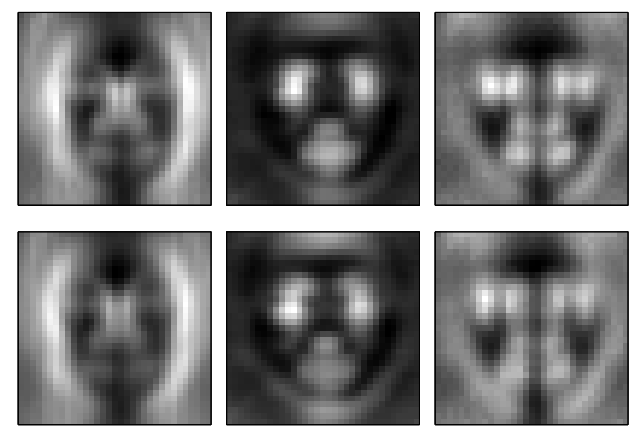

Figure 4.3: Average wavelet features over the training region.

Below, we motivate the choice of our three different feature sets. For brevity, we refer to double-res "scale-2" or "scale-4" features whenever we mean Haar wavelets that are at the double of the resolution that would be required for a complete basis (Papageorgiou and Poggio, 2000); the same holds for quad-res "scale-4" features.

In all cases, cluster-weighted models as described in Subsection 4.3.3 require a feature space with a reasonably small dimensionality. Therefore, principal component analysis (PCA) is used to reduce the dimensionality of the feature space (Duda, Hart, and Stork, 2001). PCA projects the raw feature vectors $\theta_{O}$ to a reduced context-validation vector $\theta_{R} \in \mathbb{R}^{16}$. A separate PCA is performed for each of the three feature types.

The decision-tree models are capable of using the original raw feature data. Therefore, no feature reduction is performed for these models. Below, we provide the necessary details of the three groups of overcomplete wavelet feature sets.

\section{Coarse representations}

Our first group of representations contains the course representations. It consists of $9 \times 9$ wavelets for a given orientation. As it is not clear in advance which wavelet scale yields the best performance, we use both single-res scale- 2 wavelets (Coarse scale 2) and double-res scale-4 wavelets (Coarse scale 4).

These representations enable us to capture details at a spatial scale larger than the face and its internal components. For instance, it captures the edges of the head and the cheeks that are descriptive for localising the face. We expect the representation to yield a low variance within the class of the context of faces, while still being sufficiently descriptive.

The coarse representations yield $9 \times 9$ wavelets for a given orientation for a window of size $19 \times 19$. As we use 3 orientations (horizontal, vertical, and diagonal), this yields a raw feature vector $\theta_{O} \in \mathbb{R}^{243}$. 


\section{Fine representations}

Our second group of representations consists of fine representations that incorporate more information on the spatial layout by using double-res scale- 2 wavelets (Fine scale 2), or quad-res scale-4 wavelets (Fine scale 4). In this way, we incorporate relations between large-scale features on a finer spatial resolution. On the one hand, the additional spatial information provides relational information that facilitates the localisation of the face. On the other hand, one might expect that incorporating the additional information increases the variance within the class of contexts of faces such that localisation becomes worse.

The fine representations yield $17 \times 17$ wavelets for a given orientation for a window of size $19 \times 19$. As we use 3 orientations (horizontal, vertical, and diagonal), this yields a raw feature vector $\theta_{O} \in \mathbb{R}^{867}$.

\section{Multiscale representation}

Our third group of representations consists of multiscale representations. It is the representation used by Papageorgiou and Poggio (2000). This representation uses double-res scale- 2 wavelets and quad-res scale- 4 wavelets. The representation incorporates a large amount of information of two different scales. Again, this representation might either improve detection by adding descriptive information, or deteriorate detection by adding too much variance within the class of facial contexts.

Both double-res scale- 2 wavelets and quad-res scale- 4 wavelets yield $17 \times 17$ wavelets for a given orientation and scale for a window of size $19 \times 19$. As we use 3 orientations (horizontal, vertical, and diagonal) at 2 scales, this yields a raw feature vector $\theta_{O} \in \mathbb{R}^{1,734}$.

\subsubsection{Training}

Training is required for the two stages in the gradient method. For the first stage, the two types of relative face locators are trained, viz. with the help of clusterweighted models or with the help of decision trees. For the second stage, object classifiers are trained for the two object detectors.

\section{Training the relative face locator based on cluster-weighted models}

The characteristics of the cluster-weighted model are described by its cluster weights, its distribution in the input space (equation 4.4), and its mapping to the output space (equation 4.5). The model is fully described by five parameters. They are (1) the cluster weights $p\left(c_{i}\right),(2)$ the clusters' input-space means $\mu_{i}$, (3) the clusters' input-space covariance matrices $X_{i}$, (4) the clusters' linear transformations (scalars $a_{i}$ and vectors $b_{i}$ ), and (5) the clusters' output covariance matrices $S_{i}$. These parameters are optimised by an iterative expectation maximisation (EM) algorithm (Gershenfeld, 1999; Verbeek et al., 2003), based on training data $\left\{\overrightarrow{x_{r}, t}\right\}_{t=1, \ldots, N}$ and $\left\{v_{t}\right\}_{t=1, \ldots, N}$. Each iteration is composed of two 
steps: an E-step and an M-step. When denoting the current iteration number $k$, these steps are defined as follows.

- E-step: computes the posterior probabilities of the clusters given the observed data:

$$
P^{k}\left(c_{i} \mid \overrightarrow{x_{r}, t}, v_{t}\right)=\frac{g^{k}\left(\overrightarrow{x_{r}, t} \mid v_{t}, c_{i}\right) g^{k}\left(v_{t} \mid c_{i}\right) p^{k}\left(c_{i}\right)}{\sum_{i=1}^{m} g^{k}\left(\overrightarrow{x_{r}, t} \mid v_{t}, c_{i}\right) g^{k}\left(v_{t} \mid c_{i}\right) p^{k}\left(c_{i}\right)} .
$$

- M-step: computes the most likely cluster parameters given the posterior probabilities as

$$
\begin{aligned}
& p^{k+1}\left(c_{i}\right)=\frac{\sum_{t=1}^{N} P^{k}\left(c_{i} \mid \overrightarrow{x_{r}, t}, v_{t}\right)}{\sum_{i=1}^{m} \sum_{t=1}^{N} P^{k}\left(c_{i} \mid \overrightarrow{x_{r}, t}, v_{t}\right)} \\
& \mu_{i}^{k+1}=<v>_{i} \equiv \frac{\sum_{t=1}^{N} P^{k}\left(c_{i}, v_{t}, v_{t}\right) v_{t}}{\sum_{t=1}^{N} P^{k}\left(c_{i}, v_{t}, v_{t}\right)}, \\
& X_{i}^{k+1}=<\left(v-\mu_{i}^{k+1}\right)\left(v-\mu_{i}^{k+1}\right)^{T}>_{i}, \\
& b_{i}^{k+1}=\left(X_{i}^{k+1}\right)^{-1}<\left(v-\mu_{i}^{k+1}\right) \vec{x}_{r}^{T}>_{i}, \\
& a_{i}^{k+1}=<\overrightarrow{x_{r}}-\left(b_{i}^{k+1}\right)^{T} \overrightarrow{x_{r}}>_{i}, \\
& S_{i}^{k+1}=<\left(\overrightarrow{x_{r}}-a_{i}^{k+1}-\left(b_{i}^{k+1}\right)^{T} v\right)\left(\overrightarrow{x_{r}}-a_{i}^{k+1}-\left(b_{i}^{k+1}\right)^{T} v\right)^{T}>_{i},
\end{aligned}
$$

where $\langle\cdot\rangle_{i}$ denotes the weighted average as defined in (4.11).

An iterative procedure as outlined above requires an initial estimate for the first iteration. The initial estimate is obtained using a $k$-means clustering model (Verbeek et al., 2003).

The choice of samples for training a cluster-weighted model is the same for each of the three feature-set groups introduced in Subsection 4.4. In each case, training is performed on a dataset of 1,885 faces. Within the context shown in Figure 4.2 , training samples are obtained at relative displacements $-8,-6,-4$, $-2,0,+2,+4,+6,+8$ in both the horizontal and vertical directions. Thus, 81 relative displacements for each face are used, which yields a total of 152,685 samples in the training set. Obviously, the feature vectors corresponding to the samples are different for each of the feature-set groups. Therefore, a separate model is trained for each of the feature sets.

Models are trained using $m=8$ clusters, as preliminary results have shown that further increasing the number of clusters does not improve performance.

Figure 4.4 shows the 8 -cluster centres in the wavelet space for each of the three feature set groups. The left panel of Figure 4.4 shows the cluster centres for the coarse scale-2 feature set. Each row corresponds to one cluster. The first three columns show the horizontal, vertical, and diagonal details for each cluster. The last column shows the cluster centre and confidence interval in the output space; the cross in the centre marks the actual location of the object, and 

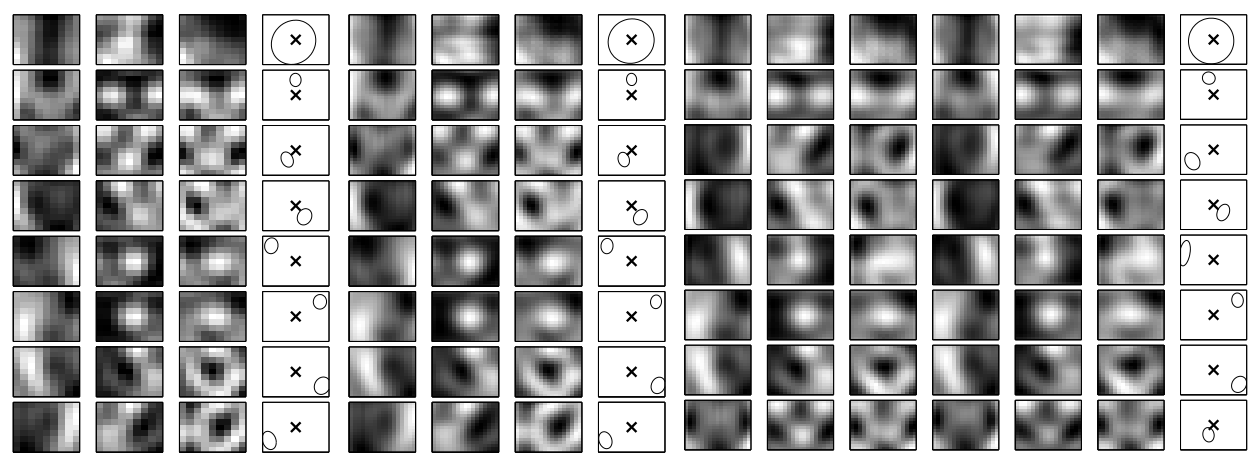

Figure 4.4: The cluster centres for the Gaussian mixture models (in wavelet space) together with their relative position and confidence intervals for the three feature sets used.

the ellipse shows a one standard-deviation confidence interval around the cluster centre. The cluster centres for the coarse scale- 4 feature set are similar.

The centre panel of Figure 4.4 shows the cluster centres for the fine scale-2 feature set. The columns have the same interpretation as in the left panel, with the exception that all details are sampled at a $17 \times 17$ resolution. The cluster centres for the fine scale- 4 feature set are similar.

The right panel of Figure 4.4 shows the cluster centres for the multiscale feature set. The first three columns show horizontal, vertical, and diagonal details at scale 2 , and the fourth to sixth columns show horizontal, vertical, and diagonal details at scale 4 . The last column again shows the relative position for each cluster.

The three figures clearly show that spatially large features both internal to the face (such as the eyes, the nose, and the mouth), and external to the face (such as the edges of the head), are used for localising the face. For instance, the second row in the right panel of Figure 4.4 shows that the presence of the eyes forms a strong indication that the current position is slightly above the position of the face. That means, the patterns shown in the second row are an indication that the upper-left corner of the current window is likely to lie in a small area above the actual upper-left corner of the face.

It is apparent that the cluster centres in the output space are similar for the three different feature sets. In addition, the corresponding cluster centres in the input space correspond to similar physical components in the three feature sets.

\section{Training the relative face locator based on decision trees}

The decision-tree algorithm used for the context-validation stage is trained on the same dataset of 1,885 faces as the cluster-weighted models. Within the context shown in Figure 4.2, the relative locations are discretised to a $5 \times 5$ grid. This yields 25 output classes as shown in Figure 4.5. Samples are obtained for the 25 relative displacements for each of the 1,885 faces. This yields a total of 47, 125 samples in the training set. Like in the previous subsection, the feature vectors corresponding 
to the samples are different for each of the feature-set groups. Therefore, a separate decision tree is trained for each feature set.

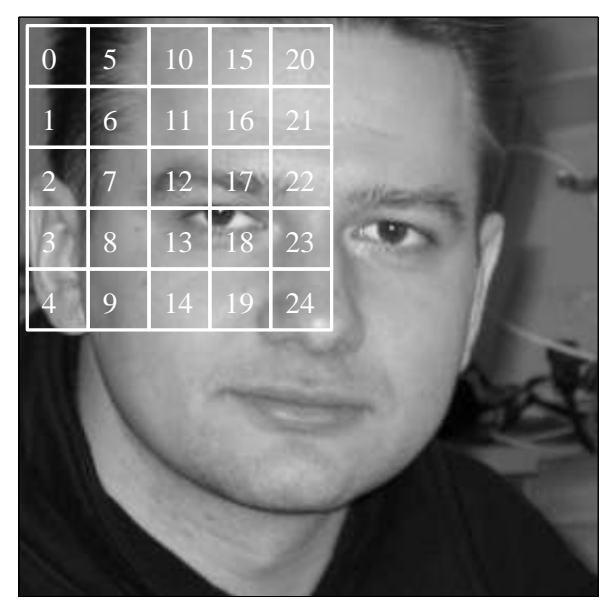

Figure 4.5: The 25 spatial relative location classes used for the decision tree.

The actual training is performed using a standard C4.5 learning scheme (Quinlan, 1993), where attribute selection and branching points are based on the information maximisation criterion.

\section{Training the object classifier}

We employ the two object classifiers introduced in Section 2.5: the monolithic detector and the boosted-cascade detector.

A SVM is used in the monolithic detector, as outlined in Subsection 4.3.1. For training we use a modification of the "boot-strap training" strategy proposed by Sung and Poggio (1998). First, an initial training set is constructed that consists of 1,900 positive instances from the AR faces database and 19,000 negative instances. These instances are obtained from random regions in the 1,421-image "training set" that is part of the natural-image set introduced in Subsection 2.3.1, and that is disjoint from the web-set. A classifier is then trained and run on another 60 images from this set, which yields 31, 613 false detections that are added to the set of negative training instances. Subsequently, a classifier is trained on this set, and it uses 2,036 support vectors.

For the boosted-cascade detector, a pre-built classifier optimised by Lienhart et al. (2002) has been employed. This classifier uses 25 stages, a window size of 24, and was trained using 5,000 positive and 3,000 negative examples per boosted stage. The output threshold on the final stage has been increased by 5 in order to obtain a reasonable false-detection rate. 


\subsection{Evaluation procedure}

The performances of the gradient methods are assessed on the web set introduced in Subsection 2.3.1. To ensure that all faces are found, the images are classified for face sizes of $24.5 \times 24.5$ pixels and up. To this end, a scale-space pyramid of the image is calculated in which subsequent scales differ by a factor 1.1 .

In order to obtain statistically valid results, we perform a 10-fold cross-validation procedure for the context classifiers. We split the dataset into 10 parts: in the model used for the localisation of faces in images belonging to set $i$, we leave out part $i$ from the training set. In addition, the model is used at different "step sizes"; at a given "step size" $s$, a PDF is calculated at only every $s$ pixels in both the horizontal and vertical direction. To determine the benefits of using our gradient method, the images are also scanned for faces in a brute-force manner by both detectors introduced in Section 2.5.

Both the cluster-weighted model based and decision-tree based context-validation methods will be applied to the raw detections of both object detectors, yielding a matrix of four experiments. The performance criteria used to evaluate the experimental results are specified in Section 2.4.

\subsection{Results}

Below we show the results of our experiments. We provide the results obtained by using the cluster-weighted models and the decision trees, both in terms of numerical results and in terms of ROC curves. In Subsection 4.6.1, we formulate observations for the results. In Subsection 4.6.2, we consider the effect of using small and large context windows.

Table 4.1 shows the detection results for the cluster-weighted method applied to the raw detections of both the boosted-cascade object classifier and the monolithic object classifier. The top five block rows show the five feature types introduced in Subsection 4.4.2. Within each block row, there are four rows corresponding to a given step size. For each combination of feature type and step size, the results for the two types of object classifiers is shown in two block columns. Within each block column, the average number of false detections per image is shown for confidence thresholds chosen in such a way that the detection rate is $80 \%, 85 \%$, and $90 \%$ respectively. In addition, the average computational time required by the context-validation stage, for images of SVGA resolution $(800 \times 600$ pixels) is shown. Note that for the monolithic classifier, results are shown for detection rates of $80 \%$ and $85 \%$ only; the monolithic classifier yields such a large number of false detections at a detection rate of $90 \%$, that the use of the detector at this detection rate is not realistic.

The bottom row in the table shows the results obtained using the brute-force object detector only.

Table 4.2 shows the detection results obtained for the decision-tree method applied to the raw detections of the boosted-cascade object classifier, and the monolithic classifier. The meaning of the rows and columns is the same as in Table 4.1 . 
Table 4.1: Detections results for the cluster-weighted method

\begin{tabular}{|c|c|c|c|c|c|c|c|c|}
\hline \multirow[b]{3}{*}{ Features } & \multirow{3}{*}{$\begin{array}{l}\text { Step } \\
\text { size }\end{array}$} & \multicolumn{4}{|c|}{ Boosted-cascade classifier } & \multicolumn{3}{|c|}{ Monolithic classifier } \\
\hline & & \multicolumn{3}{|c|}{ FD rate at det. rate: } & \multirow{2}{*}{$\begin{array}{l}\text { Val. } \\
\text { time (s) }\end{array}$} & \multicolumn{2}{|c|}{ FD rate at det. rate: } & \multirow{2}{*}{$\begin{array}{l}\text { Val. } \\
\text { time (s) }\end{array}$} \\
\hline & & $80 \%$ & $85 \%$ & $90 \%$ & & & & \\
\hline \multirow[t]{4}{*}{ Multiscale } & 1 & 0.094 & 0.215 & 0.803 & 7.94 & 1.343 & 12.546 & 34.9 \\
\hline & 4 & 0.091 & 0.224 & 0.781 & 0.78 & 1.641 & 12.953 & 3.07 \\
\hline & 8 & 0.134 & 0.279 & 0.887 & 0.34 & 3.211 & 19.433 & 1.20 \\
\hline & 12 & 0.174 & 0.348 & 1.045 & 0.17 & 4.446 & 21.829 & 0.61 \\
\hline \multirow{4}{*}{$\begin{array}{l}\text { Scale } 2 \\
\text { fine }\end{array}$} & 1 & 0.125 & 0.226 & 0.742 & 6.08 & 0.945 & 11.045 & 28.0 \\
\hline & 4 & 0.067 & 0.161 & 0.685 & 0.57 & 1.312 & 13.201 & 2.09 \\
\hline & 8 & 0.103 & 0.215 & 0.864 & 0.21 & 2.606 & 17.130 & 0.67 \\
\hline & 12 & 0.142 & 0.295 & 0.875 & 0.16 & 3.554 & 20.833 & 0.40 \\
\hline \multirow{4}{*}{$\begin{array}{l}\text { Scale } 4 \\
\text { fine }\end{array}$} & 1 & 0.117 & 0.278 & 0.850 & 5.87 & 1.646 & 14.317 & 22.8 \\
\hline & 4 & 0.119 & 0.268 & 0.800 & 0.66 & 1.982 & 15.302 & 2.23 \\
\hline & 8 & 0.175 & 0.331 & 0.793 & 0.21 & 3.557 & 18.586 & 0.69 \\
\hline & 12 & 0.191 & 0.375 & 0.977 & 0.14 & 4.650 & 19.608 & 0.42 \\
\hline \multirow{4}{*}{$\begin{array}{l}\text { Scale 2 } \\
\text { coarse }\end{array}$} & 1 & 0.076 & 0.165 & 0.730 & 8.89 & 0.741 & 12.861 & 36.6 \\
\hline & 4 & 0.096 & 0.211 & 0.728 & 0.81 & 1.483 & 11.796 & 2.40 \\
\hline & 8 & 0.121 & 0.219 & 0.735 & 0.28 & 3.105 & 17.712 & 0.64 \\
\hline & 12 & 0.159 & 0.313 & 0.909 & 0.14 & 4.662 & 19.446 & 0.34 \\
\hline \multirow{4}{*}{$\begin{array}{l}\text { Scale } 4 \\
\text { coarse }\end{array}$} & 1 & 0.118 & 0.269 & 0.756 & 5.00 & 23.483 & 24.951 & 17.9 \\
\hline & 4 & 0.143 & 0.281 & 0.812 & 0.43 & 23.483 & 24.951 & 1.30 \\
\hline & 8 & 0.172 & 0.305 & 0.883 & 0.15 & 23.483 & 24.951 & 0.45 \\
\hline & 12 & 0.163 & 0.349 & 0.974 & 0.08 & 23.483 & 24.951 & 0.23 \\
\hline Brute-force & - & 0.296 & 0.465 & 1.135 & - & 13.21 & 24.87 & - \\
\hline
\end{tabular}

Table 4.2: Detections results for the decision-tree method

\begin{tabular}{|c|c|c|c|c|c|c|c|c|}
\hline \multirow[b]{3}{*}{ Features } & \multirow{3}{*}{$\begin{array}{l}\text { Step } \\
\text { size }\end{array}$} & \multicolumn{4}{|c|}{ Boosted-cascade classifier } & \multicolumn{3}{|c|}{ Monolithic classifier } \\
\hline & & \multicolumn{3}{|c|}{ FD rate at det. rate: } & \multirow{2}{*}{$\begin{array}{l}\text { Val. } \\
\text { time } \\
\text { (ms) }\end{array}$} & \multicolumn{2}{|c|}{ FD rate at det. rate: } & \multirow{2}{*}{$\begin{array}{l}\text { Val. } \\
\text { time } \\
\text { (ms) }\end{array}$} \\
\hline & & $80 \%$ & $85 \%$ & $90 \%$ & & $80 \%$ & $85 \%$ & \\
\hline \multirow[t]{4}{*}{ Multiscale } & 1 & 0.048 & 0.075 & 0.408 & 248 & 0.385 & 14.223 & 1,105 \\
\hline & 2 & 0.057 & 0.112 & 0.588 & 110 & 0.952 & 17.861 & 449 \\
\hline & 3 & 0.077 & 0.136 & 0.708 & 83.6 & 1.437 & 18.909 & 295 \\
\hline & 4 & 0.117 & 0.217 & 0.753 & 74.3 & 3.357 & 22.918 & 258 \\
\hline \multirow{4}{*}{$\begin{array}{l}\text { Scale } 2 \\
\text { fine }\end{array}$} & 1 & 0.034 & 0.074 & 0.384 & 125 & 0.305 & 15.132 & 574 \\
\hline & 2 & 0.054 & 0.105 & 0.474 & 59.3 & 0.645 & 19.038 & 208 \\
\hline & 3 & 0.050 & 0.116 & 0.646 & 46.6 & 1.259 & 19.991 & 150 \\
\hline & 4 & 0.102 & 0.194 & 0.841 & 41.4 & 2.596 & 20.895 & 101 \\
\hline \multirow{4}{*}{$\begin{array}{l}\text { Scale } 4 \\
\text { fine }\end{array}$} & 1 & 0.061 & 0.130 & 0.641 & 137 & 0.541 & 17.502 & 544 \\
\hline & 2 & 0.090 & 0.176 & 0.697 & 65.2 & 1.242 & 19.384 & 229 \\
\hline & 3 & 0.085 & 0.177 & 0.761 & 51.6 & 2.485 & 18.854 & 167 \\
\hline & 4 & 0.150 & 0.282 & 0.968 & 45.9 & 3.468 & 22.439 & 143 \\
\hline \multirow{4}{*}{$\begin{array}{l}\text { Scale } 2 \\
\text { coarse }\end{array}$} & 1 & 0.043 & 0.088 & 0.437 & 87.2 & 0.192 & 18.701 & 370 \\
\hline & 2 & 0.067 & 0.124 & 0.470 & 50.6 & 0.794 & 18.823 & 153 \\
\hline & 3 & 0.049 & 0.129 & 0.576 & 45.3 & 1.208 & 18.512 & 111 \\
\hline & 4 & 0.133 & 0.227 & 0.849 & 41.7 & 2.608 & 20.876 & 100 \\
\hline \multirow{4}{*}{$\begin{array}{l}\text { Scale } 4 \\
\text { coarse }\end{array}$} & 1 & 0.061 & 0.154 & 0.592 & 89.2 & 0.434 & 19.007 & 321 \\
\hline & 2 & 0.098 & 0.182 & 0.654 & 53.0 & 1.137 & 17.626 & 167 \\
\hline & 3 & 0.111 & 0.232 & 0.846 & 45.4 & 1.804 & 16.147 & 141 \\
\hline & 4 & 0.162 & 0.326 & 0.998 & 42.0 & 4.334 & 21.806 & 124 \\
\hline Brute-force & - & 0.296 & 0.465 & 1.135 & - & 13.21 & 24.87 & - \\
\hline
\end{tabular}


The results in Tables 4.1 and 4.2 provide a numerical overview over all methods and parameters, at sensible detection rates. In order to show the full results for the best-performing methods, Figure 4.6 shows ROC curves for the best-performing cluster-weighted and decision-tree methods compared to the brute-force object classifiers. In contrast to the tables, the ROC curves are grouped by the object classifier, as this makes a graphical comparison more straightforward. The top panel of Figure 4.6 shows the detection results based on the object candidates obtained using the boosted-cascade classifier. The bottom panel of Figure 4.6 shows the detection results based on the object candidates obtained using the monolithic classifier. It should be noted that the horizontal scales on both plots is different, because the object-detection performance of both object detectors differs.

\section{Main result}

The main result is that the detection-performance, measured as the number of false detections per image at a given detection rate, can be reduced by a large factor when compared to the brute-force object classifier. The best result, in this regard, is obtained for the decision-tree method using scale-2 fine features, using a step size of 1 , and based on the object candidates of the boosted-cascade classifier. Here, the number of false detections per image, at a detection rate of $80 \%$, is reduced from 0.296 to 0.034 : a factor 8.7 , and thus almost an order of magnitude.

\subsubsection{Observations}

Based on the results, we formulate six observations. The first observation refers to the quality of the brute-force object classifiers. The boosted-cascade object classifier significantly outperforms the monolithic classifier. This is consistent with findings in the literature (Papageorgiou and Poggio, 2000; Viola and Jones, 2001b). Training by using a boosted cascade allows one to use a very large number of negative samples in the training (in the order of hundreds of millions to billions). This is advantageous, because object detection is a skewed problem, i.e., a natural image contains many more real negative windows than real positive windows. This difference in performance between the brute-force object classifiers is also apparent in the final results after contextual validation. It seems to indicate that a reasonably good object detector in the first gradient-method stage is a prerequisite to obtaining a good final detection result.

The second to fifth observations refer to our research question RQ1b, as stated in Section 4.1. The second observation is that for all experimental parameters, the decision-tree methods outperform the cluster-weighted methods. This observation will be discussed at length in Subsection 5.2.2.

The third observation is that contextual validation using decision trees is 32 to 102 times (about two orders of magnitude) faster than the contextual validation using cluster-weighted models. There are two main reasons for this difference in speed. The first reason is that the evaluation of the cluster-weighted model, for a 

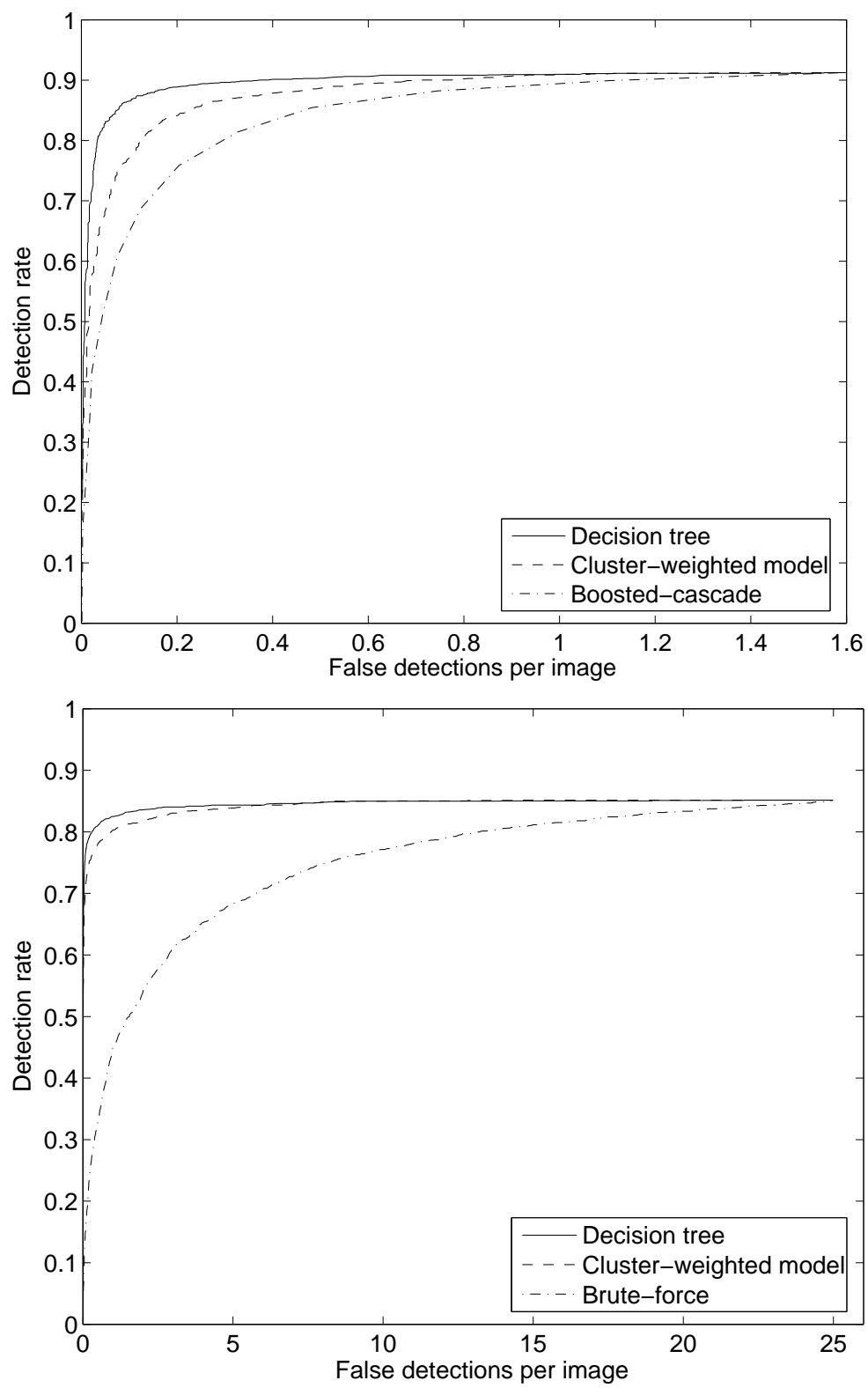

Figure 4.6: Receiver Operating Characteristic for both gradient methods based on raw object detections of the boosted-cascade classifier (top) and the monolithic classifier (bottom).

given window, requires the projection of the features to a 16-dimensional subspace (involving $16 \times 1,734$ floating-point multiplications for the multiscale feature set), which is computationally expensive. In contrast, the evaluation of a decision 
tree requires only the look-up of a moderate number of features, which, given that decision trees are reasonably shallow, is computationally cheap. The second reason is that in order to calculate a per-window PDF for a cluster-weighted model, one needs to evaluate both the Mahalanobis distance (cf. Gyorfi, Devroye, and Lugosi (1996)) to the expected location, and an expensive exponential calculation, for each pixel in the PDF. In contrast, for decision trees the PDF is obtained from the 25 posterior class probabilities using a straightforward table look-up.

The fourth observation is that for all experimental parameters, the use of scale2 features yields better detection results than the use of scale- 4 features. This is an interesting result, as one might expect that larger-scale features have a smaller intra-class variance than smaller-scale features. This point will be discussed further in Section 4.7.

The fifth observation is that the results obtained with features on scale 2 only are similar to those obtained using the multiscale feature set in case of the cluster-weighted method. In the decision-tree method, using the scale-2 features only yields a better performance than using the multiscale feature set. Together with the previous observation, it seems to indicate that scale- 4 features indeed are not very informative for contextual validation.

The sixth observation is that the detection results become worse as the step size increases. This is to be expected, as for a larger step size, one effectively uses less samples around an object candidate to obtain a confidence value. Using a larger step size does have a speed advantage, as using a step size of $n$ reduces the number of contextual model evaluations by a factor of $n^{2}$. Furthermore, the clusterweighted method is capable of obtaining good results for larger step sizes than the decision-tree method. This difference will be discussed in Subsection 5.2.2.

\subsubsection{Small windows compared to large windows}

Below, we address our research question RQ2 on how large our context needs to be. We refer the reader to an earlier discussed on a related topic in Subsection 3.6.2. Here, we assess the effect of the window size used for contextual validation. The experiments outlined above correspond to a small window size, i.e., the window has the same size as the face itself, and the training samples are taken from a region laterally twice as large. The large-window experiments outlined below, use a PPcon-sized window, that is twice as large as the face (as shown in Figure 3.2). The training region from which samples are taken is twice as large as the PPconsized window. Experiments have been performed using the same set-up as for the small-window experiments. Results for the multiscale feature set are listed in Table 4.3. Figure 4.7 shows ROC curves for a step-size equal to one.

The results give rise to two observations. The first observation is that for all experimental parameters, the large-window methods perform less than the smallwindow methods. The most probable reason for this fact, is that the intra-class variance of an object's context is larger than that of the object itself.

The second observation is that the performance of the cluster-weighted method suffers less from using a large window size than the performance of the decisiontree method. Specifically, the large-window cluster-weighted method outperforms 
Table 4.3: Detection results for the large-window gradient methods compared to those of the small-window gradient methods. Both are based on raw detections from the boosted-cascade detector.

\begin{tabular}{l|l|l|l|l|l|l|l}
\multirow{3}{*}{ Model } & \multirow{2}{*}{ Step } & \multicolumn{3}{|c|}{ Large window } & \multicolumn{3}{c}{ Small window } \\
\cline { 3 - 8 } & size & $80 \%$ & $85 \%$ & $90 \%$ & $80 \%$ & $85 \%$ & $90 \%$ \\
\hline Cluster- & 1 & 0.192 & 0.352 & 0.859 & 0.094 & 0.215 & 0.803 \\
weighted & 4 & 0.174 & 0.337 & 0.854 & 0.091 & 0.224 & 0.781 \\
& 8 & 0.215 & 0.384 & 0.907 & 0.134 & 0.279 & 0.887 \\
& 12 & 0.218 & 0.368 & 0.860 & 0.174 & 0.348 & 1.045 \\
\hline Decision- & 1 & 0.277 & 0.584 & 1.414 & 0.048 & 0.075 & 0.408 \\
tree & 2 & 0.283 & 0.531 & 1.301 & 0.057 & 0.112 & 0.588 \\
& 3 & 0.306 & 0.492 & 1.261 & 0.077 & 0.136 & 0.708 \\
& 4 & 0.278 & 0.455 & 1.248 & 0.117 & 0.217 & 0.753 \\
\hline
\end{tabular}

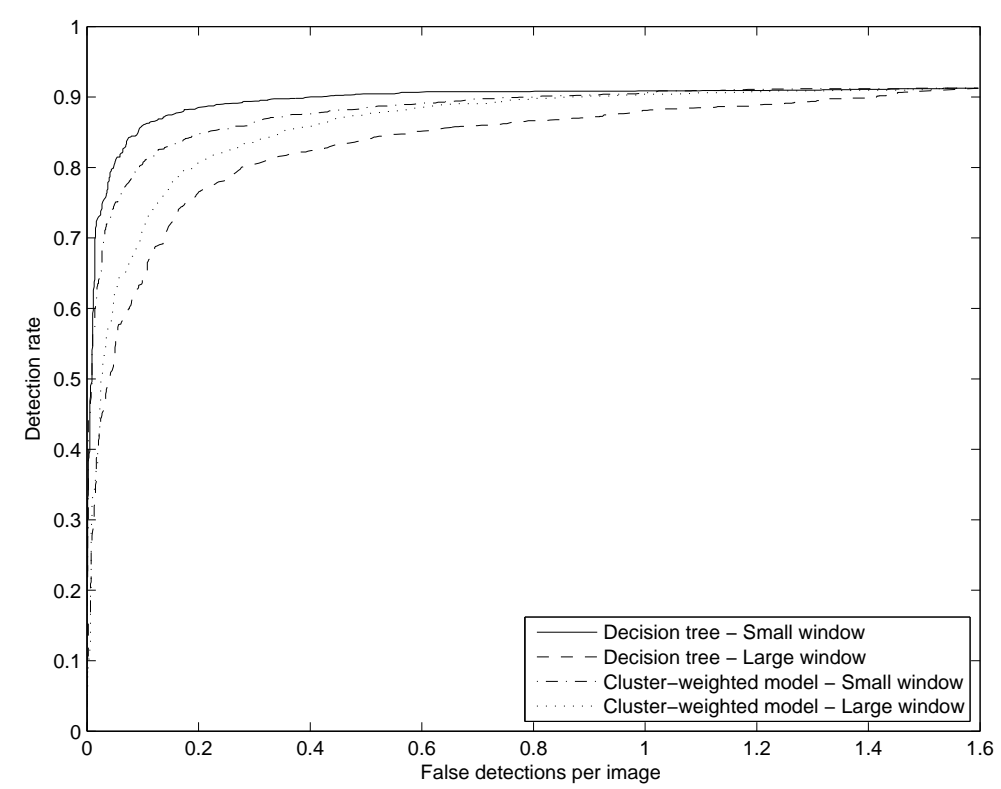

Figure 4.7: Receiver Operating Characteristic for the small-window and large-window variants of the decision-tree and cluster-weighted models.

the large-window decision-tree method, even though the decision-tree method is superior for small window sizes. This finding, as well as the previous one, will be discussed in the next section. 


\subsection{Discussion}

In this section, we discuss our results, and how they answer our research questions. In Subsection 4.7.1, we discuss the trade-off between detection accuracy and speed. In Subsection 4.7.2, we discuss the differences in performance between using large and small windows. In Subsection 4.7.3, we discuss issues related to feature types and feature normalisation. Subsequently, in Subsection 4.7.4, we discuss a basic assumption underlying the gradient method. Finally, in Subsection 4.7.5, we discuss possible extensions to the gradient method.

\subsubsection{Detection accuracy and speed}

Our research question RQ1a is whether the gradient method -by virtue of using knowledge of relative locations, would enable us to lower the number of false detections. The results show that a considerable reduction of false detections is indeed possible, but that the price to be paid for this is an increase in computation time. The reason for this increase is that the contextual validation takes place after a complete object-detection stage has been performed. So, the total computation time will always be larger than that of a complete object-detection stage.

As stated in Section 2.2, the order of the context and object stages is an implementation issue. Thus one could wonder whether a faster method could be obtained by reversing the order of the stages in the gradient method. It means that the system would first obtain a probability measure over the entire image for all objects, and would then use an object detector in high-probability locations only. Although this order would yield the same results, preliminary experiments indicate that using the context stage first requires so many decision-tree evaluations, that the context stage would take longer than the current object-detection stage.

\subsubsection{Small versus large windows}

Our research question RQ2 as stated in Section 4.1 is: what is the optimal context size?. We observed that the use of small context windows yields a better detection quality than the use of large context windows (see Subsection 4.6.2). This finding is consistent with the findings in Chapter 3.

For cluster-weighted models, we analysed the features associated with both window sizes to investigate the different results. Figure 4.8 depicts typical PCA components obtained with the small-window (top row) and large-window contextdetection methods. In general, the small-window components reveal more objectlike shapes and patterns than the large-window components. For instance, in the first column the small-window component (top) contains a small circle against a larger white-black transition, whereas the large-window component (bottom) contains a white-black transition only. The occurrence of small details in the small-window components enhances the ability of the associated classifier to detect likely object contexts. Due to their size, the large-window components tend to reflect the principal components of natural images which look like Gabor filters 


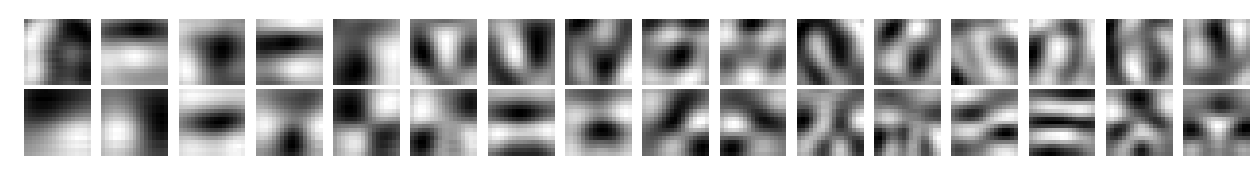

Figure 4.8: PCA components 1 to 16 for the scale-2 horizontal wavelets. Top: Normal gradient method. Bottom: Context gradient method.

(Baddeley and Hancock, 1992). Apparently, the small-window components are more descriptive for our detection task than the large-window components.

For decision trees, we observed that they suffer more from using a large window than the cluster-weighted models, to the point that the large-window clusterweighted method outperforms the large-window decision-tree method (see Table 4.3). This, too, can be explained by the larger intra-class variance, and the fact that the decision-tree models make binary decisions based on a single cut-off value of a feature/attribute, whereas the cluster-weighted models have a more continuous response to small changes in features.

\subsubsection{Feature types and normalisation}

In this subsection, we continue to address the research question RQ1b, and discuss what features are optimal for our gradient method. This question consists of two components, which will be answered below: (1) what feature scales are optimal? and (2) which type of feature normalisation is optimal?

\section{Feature scales}

In our selection of features for the region-selection stage in Subsection 4.4.2, we claimed that the coarse representations contain sufficient contextual information, without too much intra-class variance. We also claimed that the other two, more complex representations (the fine and multiscale representations) would deteriorate the results because of the incorporation of too much intra-class variance.

Our results of Table 4.2 show that the methods using the multiscale, or the scale-2, representations outperform the scale- 4 representations. In particular, both scale-2 representations - including the coarse scale-2 representation- outperform the multiscale representation. The results suggest that scale-2 representations are more informative than scale- 4 representations. This explains the superior performance of the fine scale-2 representation. The fact that the multiscale representation does not outperform the coarse scale-2 representation is probably due to the high dimensionality $\left(\mathbb{R}^{1,734}\right)$ of the feature space which, together with the limited number of available samples, hampers the generalisation performance of the classifier.

\section{Feature normalisation}

A considerable advantage of the decision-tree models over the cluster-weighted models is the time required for the context-validation stage. However, most of 
the computation time is taken by the calculation of all features. Calculation of all features is necessary because of the manner in which the features are normalised. Each feature-vector component (for a given scale and orientation) is normalised in such a way that its sum is 1 . The above type of normalisation requires that all features are calculated before any normalisation can be performed. Clearly, this is a slow method. Now we will consider the C4.5 decision trees that we use. They are rather sparse, i.e., for any path from the tree's root to one of the leaves one needs the value of only a few features. A large speed improvement could thus be obtained when using a normalisation method that does not require the precalculation of all features, but of only those that are required in each decision node in the tree on a given path from the root to a leaf.

An interesting class of methods normalises each feature by the pixel-value standard deviation or the variance of the window for which a PDF is calculated. These methods would be interesting because the calculation of the standard deviation or variance over a given window in an image can be performed in $O(1)^{3}$ in an integral-image framework (Viola and Jones, 2001b).

Preliminary experiments have been carried out to assess whether feature normalisation based on standard deviation or variance is feasible. As the combination of the decision-tree method applied to the raw detections of the boosted-cascade detector yielded the best results in the previous experiments, the preliminary experiments have been confined to this combination. In addition, the experiments have been confined to the multiscale feature set.

Table 4.4 shows detection results when normalising by standard deviation and variance, respectively. The corresponding results from Table 4.2 are shown in italics in Table 4.4. Clearly, the detection results for standard-deviation normalised features and variance-normalised features are worse than those obtained earlier. We investigate a possible reason for these worse results.

A possible reason is that while it seems natural to normalise features that are absolute values, by their sum, it seems unnatural to normalise absolutised features by a variance; the feature-value distribution will be one-sided. Therefore, we performed experiments in which the wavelet values are used as features directly (rather than first taking the absolute value). These results are listed in the bottom halve of Table 4.5. It is clear that for the standard-deviation normalised and variance-normalised experiments, these results are better than those in the top halve of Table 4.5. However, the original absolutised component-sum-normalised features still perform better. Figure 4.9 provides the ROC curves for the decisiontree method, using several different feature-normalisation methods.

We conjecture that the worse performance is due to the fact that the pixel variance (or standard deviation) is more sensitive to high-frequency components of the image, whereas the larger-scale wavelet features are less sensitive to these components. We note that these high-frequency components are not necessarily noise, but also constitute graphical features that are not specific to the object class of interest.

\footnotetext{
${ }^{3}$ Both independent of the the number of features in the window, and independent of the window's scale.
} 
Table 4.4: Detection results for absolute-value feature normalisation methods. All results are obtained using the decision-tree method with multiscale features, based on the raw object detections of the boosed-cascade classifier.

\begin{tabular}{l|l|l|l|l} 
Normalise & Step & \multicolumn{3}{|c}{ FD rate at det.rate } \\
by & size & $80 \%$ & $85 \%$ & $90 \%$ \\
\hline Pixel & 1 & 0.407 & 0.566 & 0.929 \\
standard & 2 & 0.298 & 0.486 & 0.907 \\
deviation & 3 & 0.311 & 0.476 & 0.920 \\
& 4 & 0.323 & 0.502 & 1.006 \\
\hline Pixel & 1 & 0.454 & 0.648 & 0.945 \\
variance & 2 & 0.438 & 0.579 & 0.890 \\
& 3 & 0.330 & 0.498 & 1.014 \\
& 4 & 0.422 & 0.548 & 0.937 \\
\hline Component & 1 & 0.048 & 0.075 & 0.408 \\
sum & 2 & 0.057 & 0.112 & 0.588 \\
& 3 & 0.077 & 0.136 & 0.708 \\
& 4 & 0.117 & 0.217 & 0.753 \\
\hline
\end{tabular}

Table 4.5: Detection results for several non-absulute-value feature normalisation methods. All results are obtained using the decision-tree method with multiscale features, based on the raw object detections of the boosed-cascade classifier.

\begin{tabular}{l|l|l|l|l} 
Normalise & Step & \multicolumn{3}{|c}{ FD rate at det.rate } \\
by & size & $80 \%$ & $85 \%$ & $90 \%$ \\
\hline Pixel & 1 & 0.199 & 0.317 & 0.699 \\
standard & 2 & 0.212 & 0.315 & 0.806 \\
deviation & 3 & 0.212 & 0.315 & 0.819 \\
& 4 & 0.252 & 0.361 & 0.912 \\
\hline Pixel & 1 & 0.218 & 0.307 & 0.662 \\
variance & 2 & 0.214 & 0.325 & 0.693 \\
& 3 & 0.191 & 0.357 & 0.868 \\
& 4 & 0.254 & 0.408 & 0.914 \\
\hline Component & 1 & 0.250 & 0.406 & 0.915 \\
norm & 2 & 0.225 & 0.458 & 1.054 \\
& 3 & 0.290 & 0.492 & 1.081 \\
& 4 & 0.297 & 0.565 & 1.114 \\
\hline
\end{tabular}

\subsubsection{The "object-is-near" assumption}

The basic assumption underlying the gradient method is that "an object is near to the current location". Our "object-is-near" assumption may give rise to a high false-detection rate because if an object is not near to the current location, the method is likely to overestimate the probability of an object being near. To investigate whether this is the case, we combined the small-window version and 


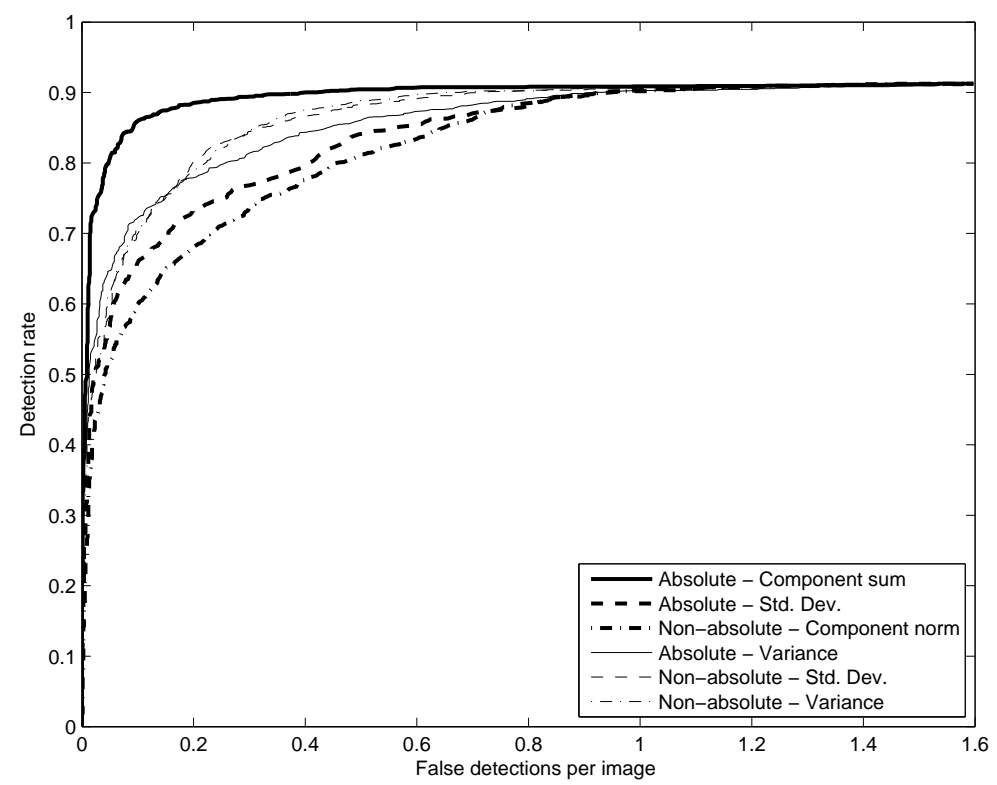

Figure 4.9: Receiver Operating Characteristic for the decision-tree method, using several different feature-normalisation methods.

large-window version of the gradient method by approximating the probability that an object is present, $P$ (object) in the following way.

$$
P(\text { object }) \approx P(\text { object } \mid \text { object in vicinity }) P(\text { context } \mid \text { context in vicinity })
$$

Figure 4.10 shows the ROC curves obtained with the gradient method using equation 4.16 -labelled "combined gradient method" - and the regular smallwindow decision-tree gradient method. Clearly, the performance for the combined gradient method is worse. Analysis revealed that the application of equation 4.16 did lower the probabilities in locations where no objects were near. However, it also lowered the probabilities in the vicinity of objects. Hence, the overall performance worsened as compared to the original gradient method. It may be possible to attenuate the probability in another way to lower the false-detection rate of the gradient method, e.g., by estimating a low-detail image-wide PDF for likely object locations using Torralba's (2002) method. We do recommend the inclusion of Torralba's method, but given the current results, we believe that our "object-is-near" assumption does not harm the accuracy too much.

\subsubsection{Extensions}

In addition to all experiments listed above, we have performed two exploratory experiments: (1) to test our assumption that small scales are most informative in 


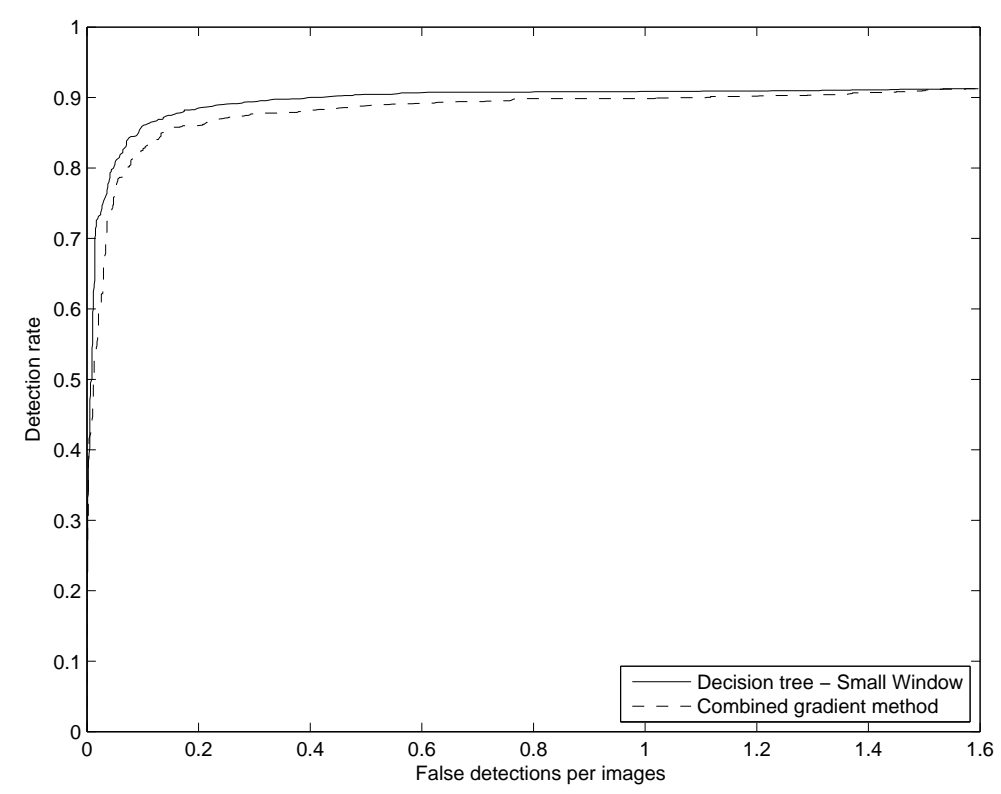

Figure 4.10: Receiver Operating Characteristic for the decision-tree method compared to the combined gradient method.

determining the relative object location and (2) extending our method to possibly alleviate the "object-is-near" assumption.

The first experiment uses a multiscale feature representation. However, unlike the previous experiments, we use wavelets at decomposition levels 2 and 3 (scales 4 and 8), rather than levels 1 and 2 (scales 1 and 2). Results are obtained using the decision-tree method, and using the same experimental set-up as before. Table 4.6 contains the results for this experiment.

\begin{tabular}{ll|l|l|l}
\hline Table 4.6: Detection results when using wavelets at levels 2 and 3. \\
\hline Step & \multicolumn{2}{|c}{ False-detection rate at det.rate } \\
size & $80 \%$ & $85 \%$ & $90 \%$ \\
\hline 1 & 0.090 & 0.190 & 0.685 \\
2 & 0.107 & 0.209 & 0.724 \\
3 & 0.123 & 0.268 & 0.748 \\
4 & 0.175 & 0.341 & 0.827 \\
\hline
\end{tabular}

The second experiment tries to alleviate the impact of the "object-is-near" assumption by adding an additional non-spatial class to the output of the decisiontree: a null-class. Whereas the first 25 classes indicate a relative location with respect to the face, the 26th class indicates that the current window is, in fact, not in the vicinity of the object. In addition to the 47,125 spatial samples, we extracted 10,000 null-class samples from the training part of the natural image 
set, in random locations that are not in the vicinity of faces. The results are shown in Table 4.7.

Table 4.7: Detection results for model with a null-class.

\begin{tabular}{l|l|l|l} 
Step & \multicolumn{3}{|c}{ False-detection rate at det.rate } \\
size & $80 \%$ & $85 \%$ & $90 \%$ \\
\hline 1 & 0.032 & 0.090 & 0.459 \\
2 & 0.055 & 0.115 & 0.566 \\
3 & 0.057 & 0.172 & 0.626 \\
4 & 0.106 & 0.194 & 0.707 \\
\hline
\end{tabular}

Figure 4.11 shows ROC curves for the results of the exploratory experiments. A step-size of 1 is used, and results are compared to those of the multiscale method from Table 4.2 .

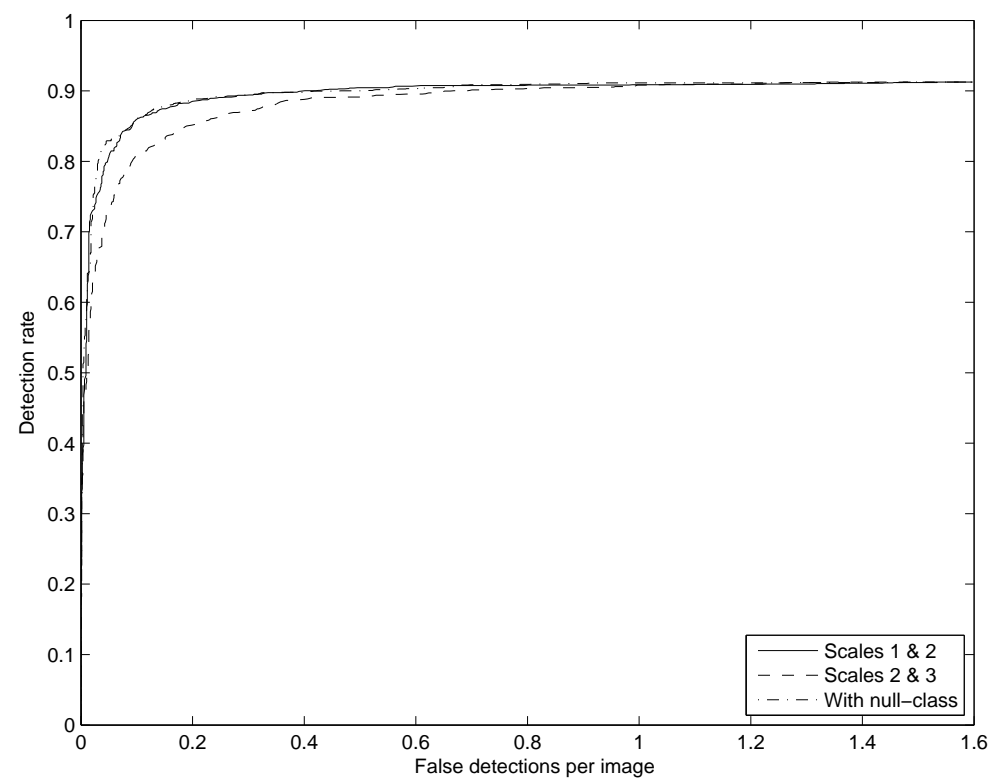

Figure 4.11: Receiver Operating Characteristic for the scale 2-3 and null-class methods, compared to the scale 1-2 method.

The results of these exploratory experiments give rise to two observations. The first observation is that the use of larger-scale features yields worse detection results than the use of smaller-scale features; an observation that is consistent with our discussion in Subsection 4.7.3.

The second observation is that the null-class method yields results that are comparable to those of the normal decision-tree method. Around a detection rate of $80 \%$, the null-class method performs slightly better. However, the extensive use of a null-class is problematic because of the skewness of the problem; the fraction 
of image location that is not in the vicinity of the object of interest, generally is much larger than the fraction of locations in the vicinity of faces.

\subsection{Chapter conclusions}

In this chapter, the second class of context-based object-detection methods the gradient method - was discussed. Our main motivation for introducing the gradient method was to reduce the dimensionality of the learning problem by taking domain knowledge into account; the relative location of small parts with respect to the object.

The results indicate that the gradient method succeeds in considerably lowering the number of false detections at a given detection rate. Therefore, we may conclude that the use of domain knowledge on relative locations improves object detection.

In addition, we may conclude that for face detection small context windows are to be preferred over large ones. With respect to the choice of classifiers, we may conclude that decision trees may be applied successfully to the image classification problems.

Whether the increased performance is purely due to the fact that we guide the learning process using the domain knowledge mentioned, is a question we will attempt to answer in the next chapter. 


\section{Chapter 5}

\section{General discussion of object detection}

This chapter is partly based on the following publication ${ }^{1}$ :

1. Bergboer, N. H., Postma, E. O., and Herik, H. J. van den (2005). Visual object detection for the cultural heritage. Proceedings of the XVI international conference of the Association for History and Computing (AHC2005), pp. 33-38, Royal Netherlands Academy of Arts and Sciences, Amsterdam, The Netherlands. ISBN 90-6984-456-7.

In this chapter, we will discuss the issues of context-based object detection that are common to both the context-detection method and the gradient-based method (henceforth called the gradient method). First, in Section 5.1, we discuss general performance considerations for both classes of methods, such as the speed versus accuracy trade-off. Then, in Section 5.2, we conduct an in-depth exploration to answer the question that concluded the previous chapter. Although we know that the gradient method succeeds in accomplishing our research goal of having fewer false detections, we would like to know the precise reason for this. In Section 5.3, we investigate whether the object-detection methods developed for natural images, can also be extended to search for objects in other manifestations of our cultural heritage, such as paintings. In Section 5.4, we discuss work that is generally related to both context-based object-detection methods we developed. Finally, in Section 5.5, we discuss possible future directions in context-based object detection, based on the findings in this chapter.

\subsection{The speed versus accuracy trade-off}

The different patterns of results obtained with the context-detection method and the gradient method arise from the different ways in which both methods trade off

\footnotetext{
${ }^{1}$ The author would like to thank his co-authors and the publisher of the AHC 2005 proceedings, for their kind permission to reuse relevant parts of the article in this thesis.
} 
accuracy and speed. The gradient method emphasises accuracy by taking multiple independent samples from the context to estimate the gradient towards the object. The enhanced accuracy is costly in computational resources as is evident from the speed results. In contrast, the context-detection method is fast for two reasons. The first reason is that it operates on a larger scale. The second reason is that it relies on an efficient Viola-Jones framework. However, the enhanced speed comes at the cost of accuracy. The reason for the detection-accuracy difference between the two methods will be explored in greater detail in the Section 5.2.

\subsection{Explaining the gradient method's performance}

Although our experiments show that the gradient method succeeds in differentiating between object detections within likely and unlikely contexts, the question that remains is: what is causing the success? We discuss three possible explanations: (1) the use of local context, (2) the relative-localisation model, and (3) the manner in which relative locations are used in training. The three possible explanations are discussed in the next three subsections.

\subsubsection{The use of local context}

The first possible explanation for the gradient method's performance is the fact that it uses local context. If the use of the local context of an object causes the successful performance of the gradient method, a similar improvement would be achieved when using a one-stage binary object detector trained on the local contextual region from which the gradient method takes its training samples. We trained such a detector (henceforth referred to as the context detector) on the $35 \times 35$ pixels training region also used for training the gradient method. We downsampled the region to $20 \times 20$ pixels because the number of features scales as $s^{4}$ with the linear size $s$ of the region. The learning algorithm employed for the context detector is taken from Lienhart et al. (2002). The classifier was trained using the same image set as the brute-force classifier. Figure 5.1 shows the detection results for the context detector, compared to the boosted-cascade detector, and the best-performing gradient method.

It is clear from the results that the performance of the context detector is inferior to that of both the boosted-cascade detector and the results obtained using the gradient methods. We therefore may conclude that purely using a local context does not explain the gradient method's improvement.

The features used for context validation may contain information that complements the information in the context detector discussed in explanation above. While it is true that our context-validation method uses features outside the central face region, the features we use are a subset of those used by the context detector. This implies that the features used for context validation are included in that context detector, and that the features do not add additional information. It is important to remark, that the gradient method employs a subset of the features used by the context detector. Apparently, the use of this subset of features leads to an improved detection performance. 


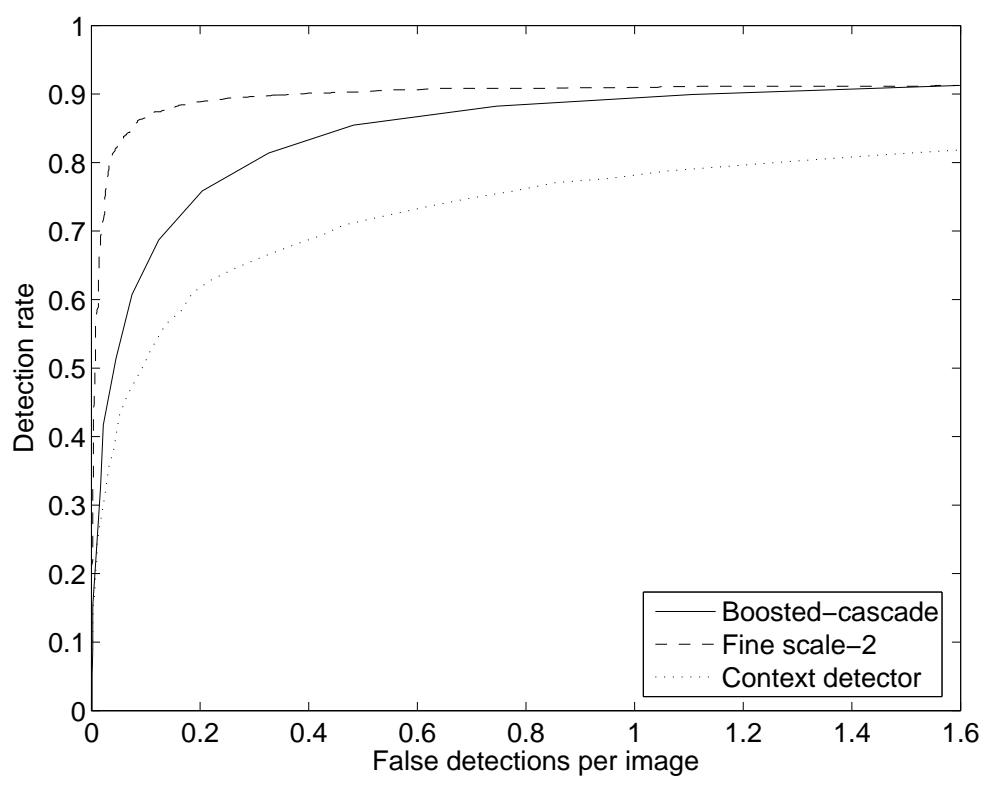

Figure 5.1: Detection results for the context detector compared to the boosted-cascade classifier and the gradient method.

\subsubsection{The relative-localisation model}

The second possible explanation for the gradient method's performance is the type of relative-localisation model it employs. The type of learning algorithm employed for inducing the contextual-validation model - cluster-weighted models or C4.5 decision trees - may explain the gradient method's successful performance. However, the types of algorithms employed in our context-validation stage have a rather different structure. Exploratory experiments indicate that a considerable detection improvement is also obtained when using naive Bayesian classifiers. We therefore believe that the type of learning algorithm is not the reason for the improvement. However, we do acknowledge that there is a significant difference in detection performance between the two relative-localisation models.

In our experiments, we observed that (1) cluster-weighted models perform well using larger step sizes than decision-tree models, and (2) decision-tree models perform better than cluster-weighted models in reducing the false-detection rate. Below, we discuss possible causes for these observations.

\section{Superior performance of cluster-weighted models using large step sizes}

The fact that cluster-weighted models are successful for larger step sizes than the decision-tree models can be attributed to the difference in output representation of both models. On the one hand, the cluster-weighted model outputs a PDF in terms of an expected relative location, and a covariance matrix. This PDF has a rather large spatial extent. Thus, even when using larger step sizes, the PDFs 
still overlap to a large degree, and the actual object location will be assigned a large confidence. On the other hand, the decision-tree models output a conditional class probability for each of the 25 spatial classes. In the majority of cases, one class will have a probability of 1 , and the rest will have a probability of 0 . This means that the "PDF" has a smaller spatial coverage than the PDF generated by a cluster-weighted model. Hence, using a larger step size will reduce the overlap of individual PDFs. As a consequence, actual object locations are assigned a low confidence. Presumably, cluster-weighted models are more resilient to increasing step sizes than decision trees, because of the large extent of their PDFs.

\section{Superior performance of decision-tree models}

The superior performance of decision-tree models as compared to cluster-weighted models may be due to the sampling density in the output space and/or the homogeneity of samples. Below, we discuss both possibilities.

First, the sampling density in the decision tree's output space (25 classes) is larger than that of the cluster-weighted model (8 clusters). In addition, preliminary experiments using a denser output sampling for the cluster-weighted model (12 clusters) show no further improvement the 8-cluster version. This suggests that the superior performance of decision-tree models over cluster-weighted models is not due to the sampling density in the output space.

Second, the decision tree has a uniform sampling over all 25 output classes. In contrast, the cluster-weighted models tend to have their cluster centres closer to the actual location of the face. Effectively, the number of local samples that contribute to the probability measure of a detection, is lower in cluster-weighted models than in decision trees. Raising the number of clusters to 12 , as alluded to above, does not change this result. The tendency of cluster-weighted models to place the cluster centres close to the actual object location may be either because of non-Gaussian properties of the data, or because of the training algorithm ending up in a local optimum. Decision trees are non-parametric learning methods, and are therefore better capable of handling non-Gaussian data. This suggests that the performance difference observed is due to the uniform sampling and the nonparametric nature of decision trees.

\subsubsection{Training using relative locations}

The third possible explanation for the gradient method's performance is the manner in which the models use information on relative locations within the object's context. However, it may be argued that the decision-tree gradient method can be transformed into the context detector introduced in Subsection 5.2.1. It is a mapping from all decision-tree (feature, class) tuples to the appropriate feature in the context detector feature set. Since the contributions of all local samples are added to obtain the final confidence for a raw object detection, it yields a one-shot classifier that predicts a confidence based on a single classification of a $35 \times 35$-pixel window. 
The gradient method differs from such a constructed one-shot classifier in two respects. The first difference is that we restrict - or steer - the learning process in such a way that we select a subset of all features in training. This helps improve generalisation. Whether our feature selection is optimal, is an open question, but the results indicate that our feature selection does improve performance. The second difference is that we normalise our features by dividing each feature vector wavelet component by the sum of its elements. Exploratory experiments indicate that this normalisation has a large impact on performance, and that normalising by the wavelet-component sum yields the best detection results.

This line of reasoning indicates that training using relative locations per se does not explain the gradient method's performance. Rather, the manner in which relative locations are used leads to an efficient feature selection, and thus the succesful performance of the gradient method. In addition, feature normalisation had been identified as an important caused for the successful performance of the gradient method.

\subsection{Broader relevance for the cultural heritage}

We developed two classes of methods to utilise the context of an object in object detection, and tested these methods on natural images. However, in a broader setting, our methods might be even more interesting when analysing visual images from our Cultural Heritage. In order to test the generalisation of our method into this domain, we test our methods on paintings.

To evaluate the performance of the context-based methods in paintings, we apply the gradient methods and the context-detection methods to a painting dataset. The painting set consists of 53 selected digitised paintings from Rembrandt and other realistic painters. The images are obtained from the WebMuseum (Pioch, 1994), and together contain 57 frontal human faces.

Table 5.1 shows the results of the best-performing decision-tree method, the best performing context-detection method (PPcon), and the brute-force boosted cascade classifier. Figure 5.2 compares the three methods in a receiver operating characteristic curve.

Table 5.1: Performance comparison of the context-based methods on the painting set

\begin{tabular}{l|l|l|l|} 
& \multicolumn{3}{|c|}{ FD rate at det. rate: } \\
\hline Detector & $80 \%$ & $85 \%$ & $90 \%$ \\
\hline Decision tree & 0.057 & 0.076 & 0.213 \\
PPcon & 0.094 & 0.151 & 0.249 \\
Boosted cascade & 0.223 & 0.893 & 1.204 \\
\hline
\end{tabular}

Strikingly, both context-based methods outperform the boosted-cascade method in detecting painted faces. A possible reason for the superior performance on painted faces as compared to natural faces, is that painters deliberately emphasise objects (such as faces) by enhancing (or even exaggerating) the contrast with 


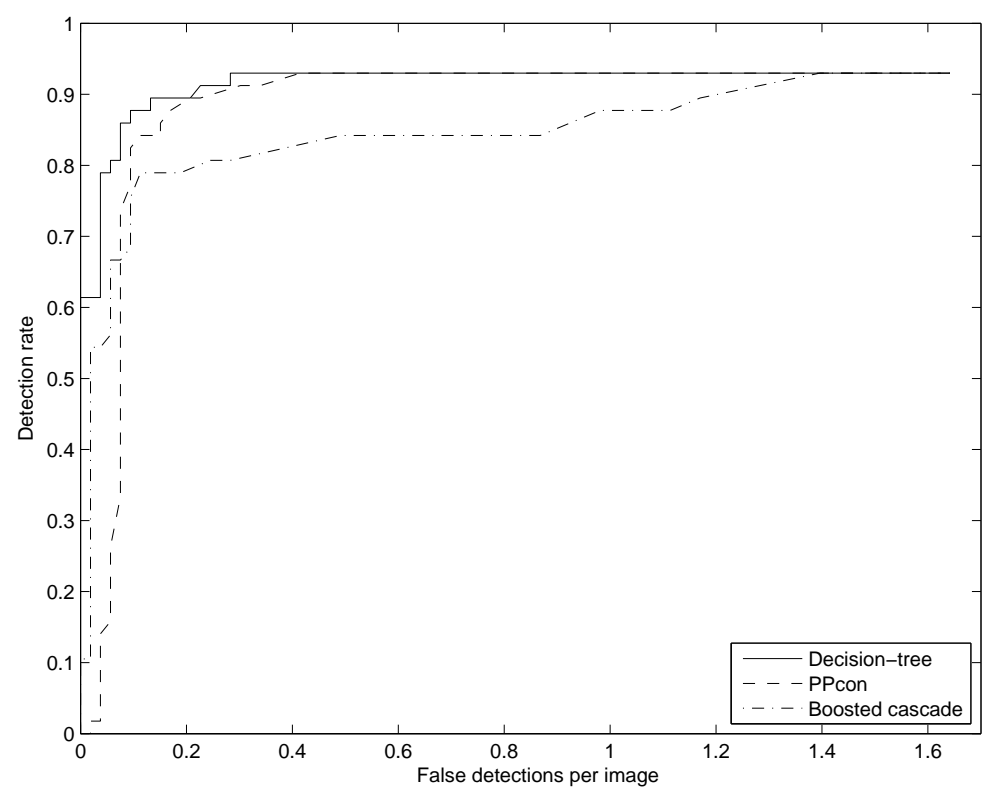

Figure 5.2: Performance comparison of the context-based methods on the painting set

the background. For instance, by enhancing the differences in spatial frequency contents between object and background (see Van Dantzig (1973) for other examples). Apparently, the enhanced contrast explains the improved performance of our methods on painted faces as compared to natural faces.

Figure 5.3 shows a typical result obtained with the decision-tree method. The image, a self-portrait by Rembrandt van Rijn painted in 1669, is shown on the left. The centre image shows all raw detections from the first detection stage. The right image displays the final detection result: the solid squares represent object detections having a sufficient confidence value, whereas the dashed squares represent object detections having an insufficient confidence value.

\subsection{Related work}

Our context-detection methods and gradient methods are related to a number of active research areas.

The context-detection method is closely related to work by Kruppa et al. (2003). They use a boosted cascade of simple classifiers to detect facial contexts that contain the head and the shoulders. In this respect, their method is similar to the context-detection method. There are two main differences between the method by Kruppa et al., and our context-based methods. First, in contrast to the context-detection method, Kruppa et al. do not use an object-detection stage in conjunction with their context detector, but infer the object location by assuming that the object is in the centre of the detected context. Second, whereas the 

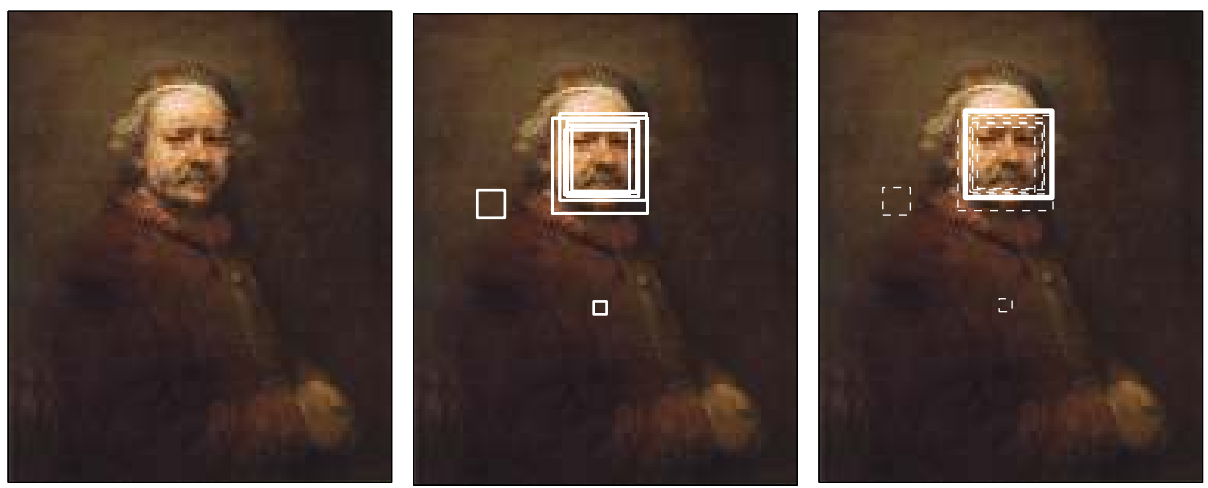

Figure 5.3: Detection example for a self portrait by Rembrandt (1669).

training region used by the small-window gradient method and context-detection method, is comparable to the context region that Kruppa et al. detect, it should be noted that the approach in the gradient method is not equivalent to head detection; whereas a head detector classifies an entire context region as either a head or not, the gradient method bases its probability measure on multiple relative location gradients obtained from windows in the vicinity of the face.

The similarities between the gradient method and existing research can be seen by regarding the gradient method as a parts-based approach, see e.g., Scheiderman and Kanade (2004). Our work is related to three other groups of parts-based approaches. Modern parts-based approaches can be broadly classified into three groups. The first group attempts to determine whether an object class is present in an image or not (Dorkó and Schmid, 2004; Lazebnik, Schmid, and Ponce, 2004), for up to more than 100 different object classes (Fei-Fei, Fergus, and Perona, 2004). The second, related, group attempts to discriminate between a number of objects classes, given that the image contains one of these classes (Fergus, Perona, and Zisserman, 2003; Helmer and Lowe, 2004). Dorkó and Schmid (2004) and Lazebnik et al. (2004) use Gaussian mixture models for parts, whereas Fergus et al. (2003), Helmer and Lowe (2004), and Fei-Fei et al. (2004) use a Bayesian framework. Even though image windows at different positions and scales can be regarded as "images", methods from the first two groups cannot be readily applied to object detection; the false-detection rate at higher detection rates $\left(\sim 10^{-3}\right)$ is several orders of magnitude too high for this purpose. The third group is more similar to our method, as these methods attempt actual object detection, i.e., the determination of the location and scale of (an a-priori unknown number of) instances from an object class (Mohan, Papageorgiou, and Poggio, 2001; Agarwal, 2002; Mikolajczyk, Schmid, and Zisserman, 2004). Our method differs from these three methods in that we avoid the problem of part selection. Rather, we choose a grid of "parts", and infer relative locations from those. In further contrast, we compare our parts-based method to a global-sampling method.

Nonetheless, techniques from these related studies may be instrumental in improving our method. The combination of different part-classifier outputs, as 
suggested in Mohan et al. (2001), is useful.

Torralba and Sinha $(2002 ; 2003)$ use sampling at a global level; they use the general spatial context, or "gist", of a scene to determine likely locations for objects in a spatially rather extended way. Torralba and Sinha extract Gabor wavelets at several orientations and frequencies from the entire area of the image, and use this information to estimate a single PDF for the image describing the target-object saliency. Their method highlights a relatively large portion of the image as a region that might contain the target object, and is thus less specific than the gradient method in locating objects. Later developments by Torralba et al. $(2003 ; 2006)$ have extended their method to perform first a scene determination, and then to use knowledge on the scene as additional prior information to determine an image-wide PDF for objects. Because the gradient method assumes a uniform prior probability over the image of finding the object, Torralba and Sinha's method can be integrated into the gradient method to weight our object confidence values, thereby effectively creating a three-stage method. This might be an attractive approach, since the gradient method makes the strong assumption that an object is present within a small distance of the current position.

Other recent techniques to obtain a statistical prior for object classes are the use of texture features from an object's context (Carbonetto, De Freitas, and Barnard, 2004; Shotton et al., 2006; Bileschi and Wolf, 2005).

In addition to the work presented in this thesis, the differences between local and global sampling approaches have also been explored in related domains of computer vision, e.g., face recognition (Heisele et al., 2003).

\subsection{Future directions}

In this section, we reflect upon the possibilities for future research, and future applications. We will first discuss future research directions that could extend our research so far. Subsequently, we discuss possible practical application areas.

\subsubsection{Future research directions}

Based on the insights we obtained in examining context-based object detection, we propose three possible future research directions.

The first future research direction concerns the use of spatio-temporal context. The problem of object detection in static images is more complex than the problem of object detection in moving imagery. This is because in moving imagery, the temporal context provides strong cues for object saliency (see e.g., Paletta and Greindl (2003)). However, given the success of using spatial context, it is recommended that a unifying framework for the use of spatio-temporal context is developed for the analysis of video data.

The second future research direction concerns the use of multiple contextual cues. From a more general point of view, our progress of the use of spatial context in static imagery can be regarded as a simple building block for future advanced content-based image-analysis methods. These future advanced methods would have to use more than just one spatial contextual cue from an image. For example, 
one could use high-level cues to determine the scene in which an image is set (Torralba et al., 2003), such as whether image portraits an indoor or outdoor scene (Payne and Singh, 2005), or one could use semantic context, such as the (statistically weak) relation between the locations of multiple objects in a scene. In addition to high-level contextual cues, low-level cues such as orientational cues could be used. Another possibility to improve advanced CBIR systems is the use of data from several modalities, e.g., to use textual meta-data in addition to the image data. Ideally, all these mechanisms should be coupled in such a way that they can check each other's functioning and can make each other's predictions more accurate.

The third future research direction concerns the problem of learning in a setting with many (contextual) cues. The main problem in combining multi-level contextual cues in one system, is that the number of samples per combination of cues is limited. First, the number of images in which a certain combination of, say, 10 cues exists, will be limited in itself. Second, the labelling of a dataset that encompasses a good statistical sample of all cues soon becomes a prohibitively expensive task. There are four possible solutions for this problem. The first solution might be the use of current and future methods that are designed specifically to learn based on small amounts of samples (e.g., Levi and Weiss (2004)). The second solution might be the use of methods that are capable of partly unsupervised learning, such as co-learning (Mitchell, 1999), and recent practical implementations thereof (Rosenberg, Hebert, and Schneiderman, 2005). The third solution might be the extensive use of domain knowledge. As we have shown in our objectdetection research, learning on a (comparatively) small number of samples can only be done when the appropriate domain knowledge is incorporated to structure the problem (i.e., reduce its dimensionality). An example of the latter is training while sharing features among object categories (Torralba, Murphy, and Freeman, 2004). The fourth solution might be to harness the experience of human observers. This can be the implicit experience in handling the outside world, that humans possess: by using human eye-movement data in the training process (Hidalgo-Soleto, Oliva, and Torralba, 2005), or by using data on human actions in handling objects (Moore, Essa, and Hayes, 1999). Alternatively, one might harness the explicit experience of humans, by having them label images or parts thereof. We mention two promising efforts in this regard. The first effort is the Google Image Labeler, which is based on the "ESP game" (Von Ahn and Dabbish, 2004). Here, the setting of a "game against the machine" is used to have two randomly paired Internet users provide a list of textual labels that describe the image that both these users see. If both users included the same word in their description list, the image is considered as labelled. This results in a database of descriptive labels for images. The second effort is Peekaboom (Von Ahn, Liu, and Blum, 2006), which can be regarded as an extension to the Google Image Labeler; Peekaboom aims at extending image labels by adding a rough position estimate for the labels. Two random Internet users are paired. One user is shown a labelled image together with the label, and has to mark the image area containing that label (e.g., "house") by mouse clicking. The other user sees only the part of the image marked by the first user, and has to guess the label based on what he sees. 


\subsubsection{Future application directions}

When advanced CBIR methods have become reality in the future, we envision many applications that will advance the access to the cultural heritage in the broadest sense. We mention two application areas that could benefit from the availability of an advanced CBIR system.

The first application area is searching in annotated photo databases. The London-based new agency Reuters files 600 photos per day on events in the news alone $^{2}$. They have a digital archive that provides commercial access to 900,000 news photos from 1995. From this, we may derive that Reuters will have an archive on many millions of (older) photos. A CBIR system could enable a more precise search for photos than the currently existing systems that use manually supplied database labels.

The second application area is searching in non-annotated, or not properly annotated, photo databases. Whereas in the Reuters example, a CBIR system could provide an increase in search quality over an existing system of high-quality manually supplied labels, CBIR could also be used in domains where manually supplied labels are either of questionable quality, or non-existent. Examples of the latter are personal photo collections and community photo sites (such as Webshots ${ }^{3}$ ). A large-scale example is the image-search function of the Mountain View based websearch company Google ${ }^{4}$. While Google provides a search functionality to search its database of over 880 million images, the search is based on text (filename and surrounding web-page context) only. A combined search based on text features and image features might enable a higher-quality search system. In fact, attempts confined to object-class discrimination have been made, e.g. by Fergus et al. (2005).

\footnotetext{
${ }^{2}$ According to their website at http://www.reuters.com

${ }^{3}$ http: //community. webshots. com

${ }^{4}$ http://www.google.com
} 


\section{Part II}

\section{Functional MRI Brain Mapping}





\section{Chapter 6}

\section{Spatiotemporal brain mapping on fMRI data}

Throughout history, scientists have been interested in the anatomy and physiology of the human body. Although the purpose and functioning of most organs has been known for centuries, the human brain has remained a mystery. Even now, the function of many parts of the brain is still unknown. Therefore, researchers in neurocognition, as well as medical professionals try to understand the workings of the brain. The $20^{\text {th }}$ century has seen the advent of numerous techniques to "see inside the brain" in vivo. In Part II of the thesis, we will concentrate on the digital analysis of data obtained using functional Magnetic Resonance Imaging (fMRI), a technique that offers an intriguing view inside the brain.

In this chapter, we introduce the problem of fMRI brain mapping, and we present the region-based method; a novel method to analyse fMRI data. In addition, we assess the region-based method's performance on experiments with simulated fMRI data. The chapter is organised as follows. Section 6.1 formulates the medical problem statement. Section 6.2 describes fMRI in detail by focussing on its relation to brain activity. In Section 6.3 the sampling of time-varying data from the fMRI data is specified in both the spatial and the temporal domain. In Section 6.4, we introduce the region-based method. In Section 6.5, we describe the experimental set-up to evaluate the performance of the region-based method. The results of applying our method to simulated fMRI data are presented in Section 6.6, and these results are discussed in Section 6.7. Finally, in Section 6.8, we draw conclusions from the results.

\subsection{The medical problem statement}

Functional Magnetic Resonance Imaging (fMRI) is an advanced non-invasive measuring technique that allows scientists and medical professionals to detect blood oxygen-density variations in the human brain and to present these variations in a three dimensional image. The brain image consists of a "time course" (fMRI 
signal over time) for every element in the 3D image, i.e., a "voxel". Through a process called fMRI brain mapping (that is explained in more detail in Subsection 6.2.1) the brain image obtained in fMRI reveals the locations and magnitudes of activation in the brain.

Existing fMRI brain-mapping methods can be broadly classified into two classes: massively univariate methods, and massively multivariate methods. We introduce massively univariate methods below. We defer the discussion of massively multivariate methods to Section 8.4. Massively univariate methods, such as the General Linear Model (GLM) (Holmes et al., 1997), treat the voxel values in the brain image as independent variables. In massively univariate methods, each voxel is analysed in isolation, without taking into account dependencies between the activities of voxels. The analysis results in a statistical measure called the specificity that indicates to what extent activation in the volume covered by the voxel is specific to an experimental manipulation. Massively univariate methods offer the advantage that they are computationally efficient and give reasonable results.

The first problem related to massively univariate methods is that they ignore possible spatial correlations between voxels. Specific stimuli or tasks tend to activate one or more distinct regions in the brain. Typically, each region corresponds to a contiguous set of voxels. Univariate methods cannot detect these spatial correlations, because the methods treat each voxel as an independent variable.

The second problem is the setting of the specificity threshold. For any given region, the specificity is often highest in the centre, and tapers off with increasing distance. The setting of a threshold on the specificity poses a problem. If the threshold is too high, the region is estimated as being too small, whereas if the threshold is too low, the region is estimated as being too large. The appropriate choice for the threshold depends on the extent of the activated region and should be determined in a context-dependent manner.

We attempt to solve both problems by introducing the region-based method. The region-based method aims at determining the specificity of brain regions by employing a machine-learning technique based on Bayesian statistics. In order to deal with the first problem, the region-based method exploits information on spatial relations between voxels, rather than estimating spatial modes across the brain. We analyse each brain location in terms of its information, and of information of other brain locations, where we limit the analysis to brain locations in the spatial vicinity of the location of interest. We thus exploit information on spatial relations within the local neighbourhood of a location in order to determine the specificity more accurately.

To address the second problem, the region-based method incorporates a scaleestimation mechanism, to determine automatically the spatial extent of an activated region without having to set a specificity threshold.

In this chapter, we present the region-based method, and we assess its performance based on experiments with simulated fMRI data. We choose to use simulated data in this chapter, as this allows us to obtain quantitative results; we will discuss this point in-depth in Subsection 6.7. Our research aims at answering the domain-specific problem statement of Subsection 1.3.2:

Domain-specific problem statement: To what extent can local 
context enhance the performance of detecting task-specific regions of activity in the brain?

Subsequently, we address two research questions from the four mentioned in Section 1.3. These are RQ3 and RQ4:

Research question 3 (RQ3): Can a new region-based method detect brain regions with high specificity on a level comparable to or better than the standard method used in the field?

Research question 4 (RQ4): Can a new method deliver a reliable scale estimate for specific regions?

The research questions RQ5 and RQ6 are specific to the use of real data and multiple test subjects, and will be formulated in the next chapter. In addition to RQ3 and RQ4, we pose a subquestion that arises form the fact that we will employ machine-learning techniques. As will become apparent, the application of machine learning techniques involves classification based on data in a very high dimensional feature space. The subquestion thus reads:

Research question 4a (RQ4a): Can a new method effectively deal with classification in a very high-dimensional feature space?

\subsection{Functional MRI and brain representation}

In this section, we provide a brief description of the process that leads from the basic MRI mechanism to the data that forms the input for our method. In Subsection 6.2.1, we briefly explain the principle of fMRI, and how it can be used to measure neural activity. In Subsection 6.2.2, we explain the general data preprocessing steps required to obtain normalised data. Finally, in Subsection 6.2.3, we state our reasons for choosing to perform our analysis on the cortical surface, rather than in the full brain volume.

\subsubsection{Functional Magnetic Resonance Imaging (fMRI)}

Functional Magnetic Resonance Imaging (functional MRI, or fMRI) is a modification of the standard MRI technique. MRI works on the basis of the physical phenomenon of nuclear magnetic resonance (Abragam and Hebel, 1961), which enables the detection of the density of single protons (mostly hydrogen nuclei in water) in a volume. As water density is a discriminative feature to distinguish between different types of tissue in the body, an MRI scan clearly shows a subject's internal anatomy.

In functional MRI, the level to which haemoglobin in the blood is oxygenated, can be measured in space and time. The crucial phenomenon that is exploited, is that haemoglobin is diamagnetic when oxygenated, and paramagnetic when deoxygenated. This means that haemoglobin modulates the MRI signal in a way 
that differs with the oxygenation level of the blood (Ogawa et al., 1990) ${ }^{1}$. This difference in modulation can be extracted from the MRI signal, in order to produce an fMRI signal.

In order to produce a brain-activation map, one exploits the fact that changes in blood flow and oxygenation in the brain (collectively known as hemodynamics) are closely linked to neural activity (Logothetis and Pfeuffer, 2004). When a region in the brain displays neural activity, it will subsequently require new oxygen. This oxygen is supplied by an influx of blood containing oxygenated haemoglobin. This so-called hemodynamic Blood-Oxygen Level Dependent (BOLD) response starts after a delay in the order of a few seconds, peaks after about 5 seconds, and then decreases back to its resting-state baseline. This BOLD response is measured using fMRI, and is - albeit indirect - a measure of neural activity.

\subsubsection{General data preprocessing}

The raw BOLD data from the fMRI scanner is delivered in the form of slice time courses (STCs). That is to say, the brain is scanned as a stack of 2-dimensional slices, and a BOLD-response time course is recorded for every pixel in every slice. In order to be able to create brain-activity maps from this raw BOLD data, three preprocessing steps have to be carried out. These preprocessing steps are performed by the BRAINVOYAGERQX ${ }^{2}$ analysis software that is used in conjunction with the fMRI scanner.

In the first preprocessing step, the data is analysed in such a way that small movements of the test subject in the scanner are taken into account. In addition, the different slices into which the brain is decomposed, are each scanned sequentially, and thus at slightly different points in time. The slice-timing differences are also taken into account. This preprocessing step results in a proper 4-dimensional (volume and time) signal.

In the second preprocessing step, a normalisation step is carried out in order to project the data into the Talairach coordinate system (Talairach and Tournoux, 1988). The Talairach coordinate system enables one to align roughly the brains from different individuals, despite differences in brain size, orientation, and differences in individual anatomy. This step is also carried out by the analysis software, based on a number of landmark points (Talairach points) that have to be identified and indicated in the brain by the experimenter. The second step results in a normalised 4-dimensional volume time course (VTC) file. At this point, one has sufficiently preprocessed the fMRI data to be able to carry out a specificity analysis in the three-dimensional brain volume. However, for reasons we will explain in Subsection 6.2.3, we choose to carry out our analysis on the cortical surface. Therefore, a third preprocessing step is required.

The third preprocessing step consists of segmenting the shape of the brain's cortex from the data. This segmentation is done automatically, based on the detection of the boundary between grey and white matter that separates the cortex from the remainder of the brain. Subsequently, the cortex representation

\footnotetext{
${ }^{1}$ In particular, the $\mathrm{T} 2{ }^{*}$ weighted MRI signal.

${ }^{2}$ http://www. brainvoyager.com
} 
extracted from each brain hemisphere is inflated to a sphere, such that surface tensions are kept to a minimum. Finally, a regular spherical grid is constructed, and the data from the volume time course are projected onto the regular grid. The third step results in a mesh time course (MTC) file, containing a time course for each vertex on the cortical surface, based on the regular spherical grid.

\subsubsection{Data selection}

As stated in the previous subsection, we perform our analysis on cortical-surface data, rather than on the full-brain volume data. There are three motivations for this choice: a neurophysiological motivation, a geometrical motivation, and an analysis motivation.

The first - neurophysiological- motivation is that the BOLD response occurs mainly in the grey matter that resides at or is close to the cortical surface. The underlying white matter consists of neural fibres that interconnect brain areas, and does not display a useable BOLD response. Restricting our analysis to the neurophysiologically relevant grey matter, implies that we exclude non-relevant data, and thus effectively increase the signal-to-noise ratio.

The second -geometrical- motivation is the following. The brain's outer surface is very much curved and folded in order to accommodate the large cortical surface area of cortical sheet. In our region-based method, we should define our regions in such a way, that the distance between two points in our representation corresponds to the distance between these two points along the cortical surface. In a volume representation, Euclidean distance does not always correspond to cortical distance. For example, two points that lie very close to each other in the 3D image, but are on opposite sides of a sulcus, will be a large distance apart when measured along a cortical surface. Figure 6.1 provides an illustration. Both panels of the figure show the same coronal section of the brain, with two points (A and B) that are on opposite sides of a sulcus. The left panel shows that the Euclidean distance between the points in the volume is small. The right panel shows that the distance between the points along the cortical surface is, in fact, large. In a surface-based approach these points will indeed lie far apart, because of the manner in which the cortex is inflated to a sphere.

The third -analysis- motivation is that the amount of data is much reduced in the $2 \mathrm{D}$ image as compared to the $3 \mathrm{D}$ image. As we will perform our analysis with machine-learning techniques, the data reduction may alleviate the curse of dimensionality (see Subsection 3.6.1), and therefore improve the generalisation performance of the region-based method.

\subsection{Sampling}

In this section, we describe the sampling of time-varying data from the $2 \mathrm{D}$ grid. In order to extract BOLD-response amplitude information, we need to sample information both temporally, and spatially. Subsection 6.3.1 describes the temporal sampling, and Subsection 6.3.2 describes spatial sampling on the cortical surface. 

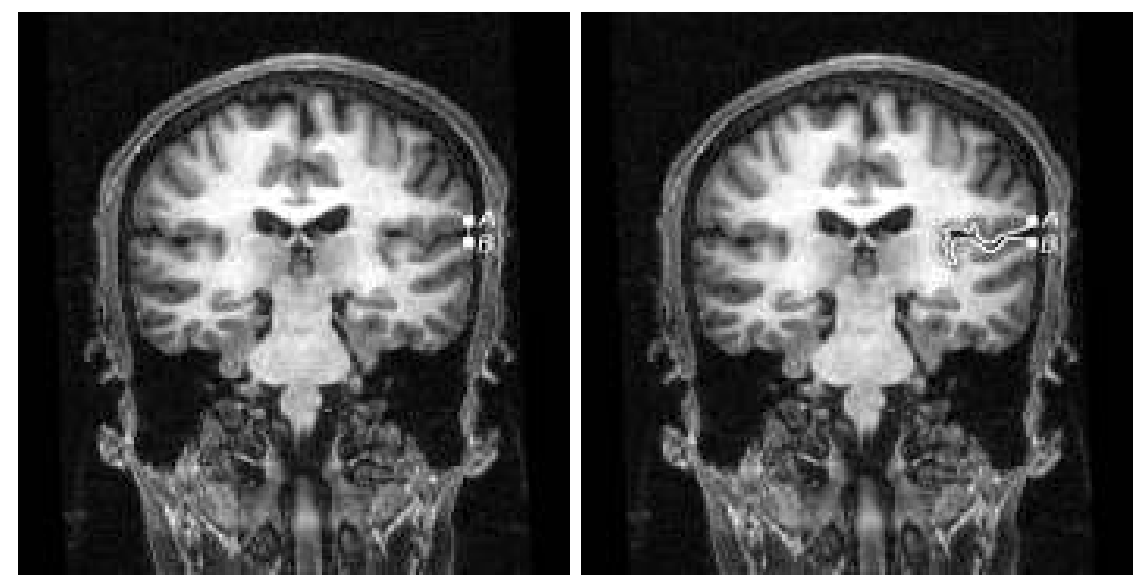

Figure 6.1: Identical coronal sections of the brain, showing two points (A and B) on opposite sides of a sulcus. The 3-dimensional Euclidean distance in the brain is small, as shown in the left section, while the distance along the cortical surface is large, as shown in the right section.

\subsubsection{Temporal sampling}

In order to perform temporal sampling, we need to define how to select a temporal portion of fMRI activity during a stimulus exposure that is informative for our goal of detecting stimulus-specific brain regions.

Figure 6.2 shows the selection of features in two commonly used experimental designs: an event-related design (left) and a block design (right). The shaded time intervals represent the sampling region. For both designs, the average activation during this time interval is defined as a measure of brain activity. In the eventrelated design the subject is exposed to short stimuli. The BOLD response in this case is an impulse response. In the block design the subject is exposed to a stimulus during a "block" of time that is substantially longer in duration than the characteristic timescale of the BOLD impulse response.

In the experiments of this Chapter, an event-related paradigm is used. That is, we will sample each stimulus exposure as indicated in the left panel of Figure 6.2. In Chapter 7, a block-design paradigm will be used.

\subsubsection{Spatial sampling: spherical coordinate indexing sys- tem}

In this section, we present the spherical indexing coordinate system that will be used as input for the region-based method. The indexing system allows for (1) an efficient spatial samples and (2) an efficient extraction of features at a subsequent stage. The spherical coordinate system is based on the regular spherical grid that is produced by BRAINVOYAGERQX in the third general data-preprocessing stage. Ideally, a regular grid would be preferred for analysis. However, as a sphere does not have a planar representation, the grid cannot be fully regular, and we define a 

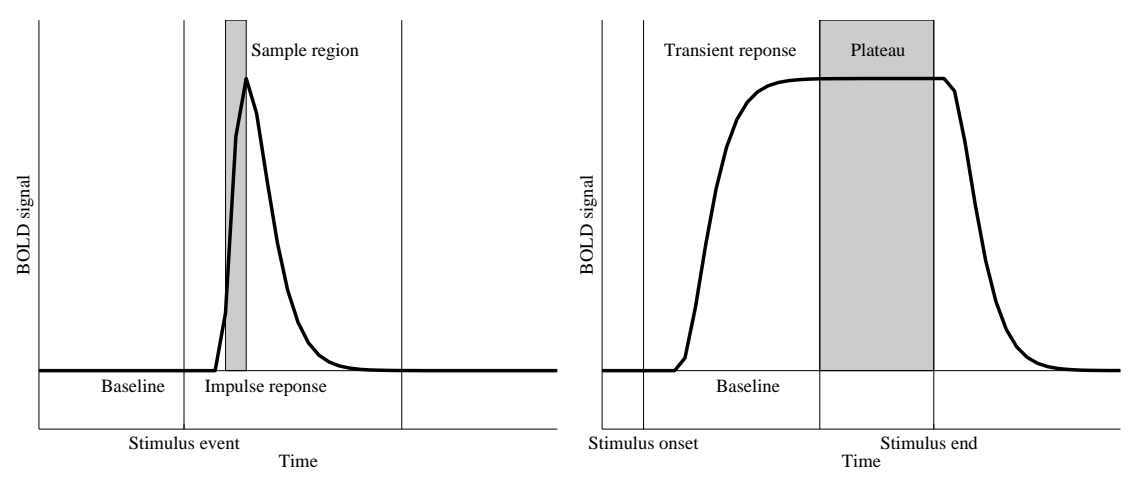

Figure 6.2: Temporal selection of features in an event-related design (left), and in a block design (right).

semi-regular grid instead. The semi-regular grid is based on an icosahedron (i.e., a Platonian solid consisting of 20 triangles, which has an approximate spherical shape). Each of the 20 triangles is recursively subdivided into four triangles, down to the desired level of detail. After each iteration, all vertices are projected outward, such that they all are at the same distance from the sphere's centre. For $n$ recursive steps, the resulting grid consists of $20 \cdot 4^{n}$ triangles.

The left panel of Figure 6.3 shows the spherical grid for $n=4$. The black lines demarcate the 20 triangles from the original icosahedron, and the grey lines demarcate the subdivided triangles. The grid is regular (i.e., at each vertex, 6 regular triangles meet), except at the 20 vertices of the original icosahedron; 5 regular triangles meet in these vertices.

The crucial point in defining a coordinate system for the sphere, is the realisation that although the spherical grid is not fully regular, the triangular grid within each of the 20 original icosahedron triangles is regular. The centre panel of Figure 6.3 shows one of these 20 triangles outlined in bold on the surface of the sphere. The regularity of the grid within each of the 20 triangles, one of which is shown in the right panel of Figure 6.3, allows us to describe a position on the sphere as a combination of a triangle number $t \in[1,20] \subset \mathbb{N}$, with the position within the triangle. We call this our spherical coordinate indexing system.

Within each triangle $t$, we define the position as a combination of the two numbers $a, b \subset \mathbb{N}$. By definition, $a$ denotes the row number within the triangle, counted from the top. That is, the top vertex has $a=1$, the two vertices on the second row have $a=2$, and so forth. The number $b$ is defined as the number of grid steps to the right, from the left edge of the triangle. That is, the top vertex has $b=1$, the two vertices on the second row have $b=1$ and $b=2$, the three vertices on the third row have $b=1, b=2$, and $b=3$, and so forth. The maximum value that $a$ can take is a function of the number of subdivisions that is made within each of the 20 icosahedron triangles. For $n$ subdivisions, $a \in\left[1,2^{n}+1\right] \subset \mathbb{N}$. The coordinate $b$, in all cases, is bounded by $b \in[1, a] \subset \mathbb{N}$. In the example shown in Figure $6.3, n=4$.

In our implementation, we define an ABT matrix that translates each valid 

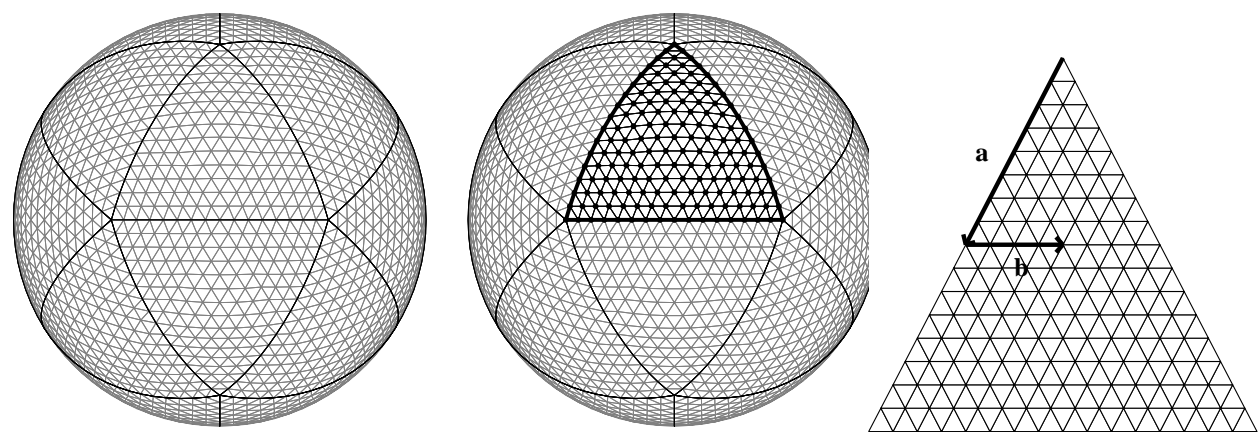

Figure 6.3: Spherical coordinate system. Left: subdivided icosahedron, centre: a single triangle, right: isolated triangle $t$ with internal coordinate system $(a, b)$.

$(a, b, t)$ coordinate to the associated vertex number on the spherical grid. This enables a translation from points in our $(a, b, t)$ coordinate system to data on the cortical surface in complexity $O(1)$.

Having outlined the temporal and spatial sampling of the fMRI signals, we now turn to the description of the region-based method.

\subsection{The region-based method}

In this section we describe our region-based method. The region-based method has two aims. The first aim is to detect brain regions that are specific to the processing of a stimulus. This detection will be performed using a machine-learning algorithm based on Bayesian statistics. A second aim is to determine automatically the spatial extent, or scale, of these specific regions. The method consists of two parts. The first part specifies the definition of features, which we introduce in Section 6.4.1. The second part specifies the machine-learning algorithm, which we introduce in Section 6.4.2. Our main contribution in the first part consists of the definition of a wavelet basis on the spherical grid defined in Section 6.3.2. Our main contribution in the second part consists of a heuristic modification of the handling of Bayesian statistics in the machine-learning algorithm, in order to cope which the large number of features.

\subsubsection{Definition of features}

In this section, we describe the features we use on the cortical surface, and how they can be extracted in a computationally efficient manner. Our contributions lie in the definition of our features, and the fast extraction mechanism. 


\section{Definition of spatial features}

In this subsection, we motivate our choice of features on the sampled cortical surface. As stated in Section 6.1, our aim is to determine the specificity of a vertex with respect to a given stimulus or experimental condition.

We will classify a vertex as specific to a stimulus (or not), depending on both the time course of that vertex itself and the time courses of vertices in its spatial neighbourhood. In order to do so, we require our features to have four properties. First, as we will estimate the scale of activated regions, the features must incorporate information from neighbourhoods at different scales. Second, we require our features to accommodate spatial averaging over a specifically sized region. We expect that averaging over multiple vertices will cancel out spatial noise, and will thus lead to a better detection performance. Third, we require our features to be able to represent the edges of specific regions, as this will likely allow us to detect the exact spatial extent of a region in a better way. Fourth, we wish to choose a feature set that can be computed in an efficient, fast manner.

A type of spatial feature that fits these requirements well, is a wavelet (Mallat, 1999). The wavelet transform realises the extraction of wavelet features from the raw signals. The wavelet transformation is based on the convolution of a function with a set of low-pass and high-pass filters, in order to split up the data in different frequency components. In this sense, it is comparable to a Fourier transformation. However, in contrast to a Fourier transformation, the filters are usually designed to be finite impulse response filters, i.e., they have a finite spatial extent. Therefore, the wavelet transform offers a clear advantage over the Fourier transform, as it makes localised analyses of the data possible. The wavelet transform can be regarded as a generalisation of the windowed Fourier transform, where the wavelet transform offers a broader choice of basis functions. The wavelet transform implements the splitting of the signal into different frequency components by using low-pass and high-pass filters at multiple spatial scales.

The fact that wavelet features satisfy the four requirements set forth above, can be seen as follows. Our first requirement is met, because wavelets can be extracted as multiple scales. Our second requirement is met, since low-pass wavelets in traditional wavelet theory correspond to features that average over a region. Our third requirement is met, since high-pass wavelets correspond to features that detect edges of specific regions. We will refer to these high-pass wavelets as "gradient wavelets" in the remainder of this chapter. There are many different wavelet types that could possibly be used, and all satisfy the first three criteria that we imposed. In order to satisfy the fourth (computational-efficiency) requirement, we choose to use a Haar-wavelet-like representation based on Viola and Jones (2001b). Below, we will introduce our wavelet features. Appendix A.1 provides more information on issues of computational complexity and efficiency.

Since we operate on the triangular $(a, b, t)$ grid as introduced earlier, instead of on a normal rectangular grid, we cannot use standard Haar wavelets. Therefore, we have to modify the Haar-wavelet definition to be compatible with our grid. Whereas there exist some definitions for wavelets on spheres (e.g., spherical harmonics (Freeden and Windheuser, 1996)), they are neither localised nor Haar-like. 
We therefore define Haar-like wavelets on our triangular grid, that satisfy the four criteria mentioned above. In addition, we choose to use a wavelet support (the outer edge of the wavelet) that, like for normal Haar wavelets, has a constant shape. In our case, we choose this shape to be a hexagonal. Below, we define four types of wavelets.

Figure 6.4 shows the four types of wavelets employed in our method, using a wavelet of scale 3 as an example. The top-left panel of Figure 6.4 shows an averaging wavelet, and the other three panels show the gradient wavelets in each of the three grid directions.
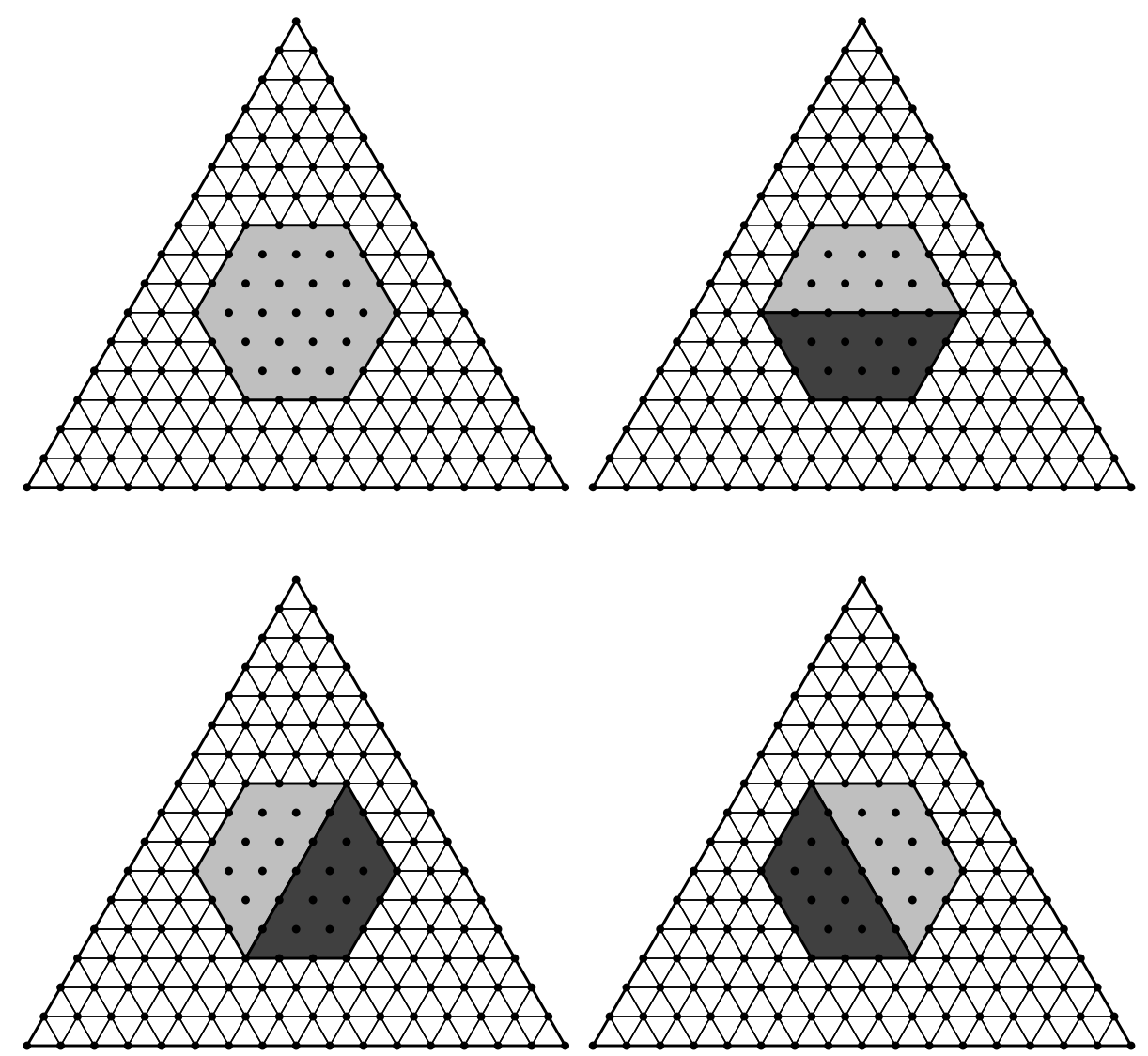

Figure 6.4: Spherical wavelet types: Averaging wavelet (top-left), and gradient wavelets in each of the three directions.

In the region-based method, we perform an overcomplete wavelet decomposition. That is, we extract the aforementioned four types of wavelets at every possible location on the spherical grid, and at a dyadic scale grid $(1,2,4$, etc.). 


\section{Extraction of instances}

In the previous subsection, we have discussed how wavelets can be extracted spatially. Since the aim of the region-based method is to detect specific brain regions using a machine-learning approach, we have to define instances for learning. In the dataset, a subject is exposed $k$ times to each of the two stimuli. For each of these exposures, we extract an instance. Following the selection criteria in Subsection 6.3.1, we average the BOLD data across the 2 th to 4 th time step after the stimulus onset, in order to obtain a spatial pattern describing a particular stimulus exposure. From this spatial pattern we extract the features introduced in the previous subsection, yielding one instance consisting of, say, $n$ features. This is repeated for each of the $k$ stimulus exposures of each of the two stimulus types. The above-mentioned process results in a dataset suitable for our machine-learning algorithm: a dataset of $2 k$ instances that each consist of $n$ features.

\subsubsection{The Bayesian algorithm}

In this section, we describe the learning algorithm of our region-based method. The algorithm is trained to map the instances defined in the previous subsection onto stimulus-specific areas of the brain. Alternatively, the machine-learning algorithm can be readily adapted to allow for the naive-Bayesian classification of brain states. In addition, the Bayesian formulation of our algorithm can be used to define a scale-estimation mechanism.

Below, we start our research by presenting the algorithm for detecting stimulusspecific regions. Subsequently, we present the adaptation to realize naive-Bayesian brain-state classification. Finally, we present the scale-estimation mechanism.

\section{Detecting stimulus-specific regions}

In the region-based method, the determination of stimulus-specific regions is treated as a learning problem in which each vertex on the cortical surface is assigned a probability of being specific to one of the stimuli. The mechanism is based on Bayesian statistics, and shares a property with the naive Bayesian framework (Mitchell, 1997); it uses the independence assumption. The mechanism incorporates a heuristic modification that we developed in order to solve the problems associated with the large number of features in a better way.

The feature and instance extraction mentioned in Subsection 6.4.1 results in $2 k$ instances, each consisting of $n$ features that represent a wavelet coefficient. Given this data, we effectively wish to compute $P\left(v_{j}\right.$ specific to $\left.c_{k}\right)$ for every vertex $v_{j}$, and for the two classes $c_{1}$ and $c_{2}$. However, in order to make numerical comparison easier, we calculate the $\log$-likelihood ratio $L\left(v_{j}\right)$ for each vertex.

$$
L\left(v_{j}\right)=\log \left(\frac{P\left(v_{j} \text { specific to } c_{1}\right)}{P\left(v_{j} \text { specific to } c_{1}\right)}\right)
$$

A value of $L\left(v_{j}\right) \gg 0$ indicates that a vertex is specific to stimulus 1 , and a value of $L\left(v_{j}\right) \ll 0$ indicates that a vertex is specific to stimulus 2. Given the 
region-based nature of our method, we determine the specificity of a vertex by using information from that vertex' local spatial context. We exploit the neurophysiological fact that a region is specific to a stimulus, if the BOLD response in that region as a result of that stimulus is significantly larger than the BOLD response in that region as a result of the other stimulus. Stated differently, a region is specific to a stimulus if the BOLD response in that region as a result of that stimulus is larger than the BOLD response averaged over both stimuli. We thus approximate the specificity measure in (6.1) by:

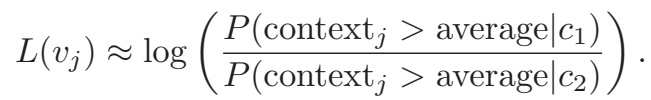

Information on whether the BOLD response in the context of vertex $v_{j}$ is larger than the average, for both stimulus classes, is obtained from the wavelet features in the local spatial context of $v_{j}$, that overlap vertex $v_{j}$. We encode this BOLDamplitude-related information by discretising each of the feature values as "above average" or "at or below average", where the average for the feature is determined across all instances. We define each feature ${ }^{3} a_{i}$ to be "true" for a given instance, if the feature for that instance is above average, where the average is taken over all instances. Furthermore, we make the assumption that the probability of a vertex' local spatial context being above average is proportional to the probability of all wavelets that overlap the vertex being above average. Having defined the set of wavelets that overlap vertex $v_{j}$ as $O_{j}$, this means we approximate $L\left(v_{j}\right)$ as follows:

$$
L\left(v_{j}\right) \approx \log \left(\frac{P\left(a_{i}\left(\forall i \in O_{j}\right) \mid c_{1}\right)}{P\left(a_{i}\left(\forall i \in O_{j}\right) \mid c_{2}\right)}\right) .
$$

As the multivariate posterior PDFs in the above equation are difficult to estimate properly, we next use the independence assumption that is also used in naive Bayesian classification. We assume that all features are independent ${ }^{4}$. We can thus approximate $L\left(v_{j}\right)$ as follows:

$$
L\left(v_{j}\right) \approx \log \left(\prod_{i \in O_{j}} \frac{P\left(a_{i} \mid c_{1}\right)}{P\left(a_{i} \mid c_{2}\right)}\right)=\sum_{i \in O_{j}} \underbrace{\log \left(\frac{P\left(a_{i} \mid c_{1}\right)}{P\left(a_{i} \mid c_{2}\right)}\right)}_{\equiv \ell\left(a_{i}\right)} .
$$

In contrast to the multivariate PDFs in (6.3), the univariate PDFs $P\left(a_{i}, c_{k}\right)$ in the above equation are readily estimated from the data. Furthermore, we note

\footnotetext{
${ }^{3}$ In literature related to Bayesian methods, it is common to use the designation "attribute" where we use the designation "feature". In order to maintain textual consistency, we use the designation "feature" throughout this thesis.

${ }^{4}$ Since the features are overlapping wavelets, this is not the case. However, Domingos and Pazzani (1996) prove that violating the feature-independence assumption for the naive Bayesian classifier does not hamper classification performance, even though probability estimates are biased.
} 
that the value defined as $\ell\left(a_{i}\right)$ in the above equation, is specific to a given wavelet feature. This value can thus be precalculated, and be reused in the calculation of the specificity of every vertex that is overlapped by wavelet $a_{i}$. The interpretation of $\ell\left(a_{i}\right)$ is as follows. If $\ell\left(a_{i}\right)$ is highly positive or highly negative, then the feature is specific to one of the stimuli. Since each stimulus is applied $k$ times, we have $k$ possible distinct values for $P\left(a_{i} \mid c_{1}\right)$ and $P\left(a_{i} \mid c_{2}\right)$. This means that $\ell\left(a_{i}\right)$ can have $4 k(k-1)$ distinct values.

The above procedure can be used to calculate the specificity likelihood ratio of every vertex. However, given that the number of instances $k$ is small in comparison with the number of features, we expect that the $P\left(a_{i} \mid c_{j}\right)$ values computed from the wavelet data are inaccurate estimates of the true probabilities. This, in turn, causes the estimates of $L\left(v_{j}\right)$ to be inaccurate.

Our approach in solving this problem is similar to that used in the training of C4.5 decision trees (Quinlan, 1993). We approximate the probabilities' likelihood ratio $L\left(v_{j}\right)$ by selecting only the most discriminative features (i.e., those having a sufficiently large absolute value of $\ell\left(a_{i}\right)$ ) into account. We expect that this selection improves the estimates of $L\left(v_{j}\right)$, because a feature having a very large value absolute of $\ell\left(a_{i}\right)$ is likely to be truly specific, even though its estimated value of $P\left(a_{i} \mid c_{j}\right)$ is somewhat inaccurate.

In practice, the approximation takes the form of a successive approximation; the most discriminative features are taken into account first, and less discriminative features are subsequently included. It should be noted that this approach is readily implemented in our method using the concept of feature grouping; given that the number of samples $k$ is small, the number of distinct values for $\ell\left(a_{i}\right)$ is limited to $k^{2}$. We therefore group features having the same value of $\ell\left(a_{i}\right)$, and rank these groups based on their absolute value of $\ell\left(a_{i}\right)$.

To make this more formal, we define $O_{j, g}$ as the features that overlap vertex $j$ and that belong to the group of features having the $g$ th largest absolute value for $\ell\left(a_{j}\right)$. Then, after taking $G$ feature groups into account, we have for each vertex:

$$
L\left(v_{j}, G\right)=\sum_{g=1}^{G} \sum_{i \in O_{j, g}} \ell\left(a_{i}\right) .
$$

The method works for wavelets that average over certain regions. Now, there are two facts we did not yet take into account. The first fact is that wavelets having a larger size contribute less information per vertex. The second fact is that, in addition to averaging wavelets, we use three types of differentiating wavelets. To incorporate both facts into our definition of the likelihood $L\left(v_{j}, G\right)$, we denote the value of wavelet $i$ at vertex $j$ as $w_{i, j}$, and the scale of wavelet $i$ as $\sigma_{i}$. The values $w_{i, j}$ are used as weights that take both the scale and the type of wavelet into account. In order to normalise the wavelets, we set their value at every vertex as inversely proportional to their scale $\sigma_{j}$. The distinction between averaging and gradient wavelets is reflected in the sign of $w_{i, j}$; a positive sign corresponds to the light parts of the wavelets as shown in Figure 6.4, whereas the negative sign corresponds to the dark part. The value of $w_{i, j}$ is thus defined as $w_{i, j}= \pm 1 / \sigma_{i}$. 
In computing the likelihood, these values are used as weighting factors as follows.

$$
\begin{aligned}
L\left(v_{j}, G\right) & =\sum_{g=1}^{G} \sum_{i \in O_{j, g}} w_{i, j} \ell\left(a_{i}\right) \\
& =\sum_{g=1}^{G} \sum_{i \in O_{j, g}} w_{i, j} \log \left(\frac{P\left(a_{i} \mid c_{1}\right)}{P\left(a_{i} \mid c_{2}\right)}\right) \\
& =\sum_{g=1}^{G} \sum_{i \in O_{j, g}} \log \left\{\left(\frac{P\left(a_{i} \mid c_{1}\right)}{P\left(a_{i} \mid c_{2}\right)}\right)^{w_{i, j}}\right\}
\end{aligned}
$$

The weighting $w_{i, j}$ can be interpreted as follows: if a feature is not specific (i.e., $P\left(a_{i} \mid c_{1}\right) \approx P\left(a_{i} \mid c_{2}\right)$ ), neither the value of the fraction $(\sim 1)$ nor the loglikelihood $(\sim 0)$ will change. For specific features, the wavelet will contribute in a positive way to $L\left(v_{j}, G\right)$ when $w_{i, j}>0$, and in a negative way when $w_{i, j}<0$. The negative contribution is appropriate: if the area covered by the negative side of a wavelet makes that wavelet very specific, it is actually the other stimulus for which that area is specific.

This completes our presentation of the machine-learning algorithm for detecting stimulus-specific regions. In our experiments, we will apply equation (6.6), in combination with our hexagonal Haar features, to the BOLD response data.

\subsubsection{Brain-state classification}

The framework underlying our vertex-classification algorithm is an interesting starting point for automatic classification of brain states. In anticipation of the application of the brain-state classification (of Chapter 7), we here present the automatic brain-state classification. In brain-state classification, a classifier attempts to classify a subject's brain state based on the features on the cortical surface, where this classifier was trained on data that is not part of the test set. The classifier's output, for each stimulus exposure, is thus a conditional probability of the subject being exposed to stimulus 1 or stimulus 2 .

Given the learned prior probabilities $P\left(a_{i} \mid c_{1}\right)$ and $P\left(a_{i} \mid c_{2}\right)$, a standard naive Bayesian classification of the brain state based on the measured features $\bar{a}_{i}$ (where each $\bar{a}_{i}$ may be "true" of "false"), calculates the following conditional probabilities:

$$
\begin{aligned}
& P\left(c_{1} \mid \bar{a}\right)=P\left(c_{1}\right) \prod_{\bar{a}_{i} \text { true }} P\left(a_{i} \mid c_{1}\right) \prod_{\bar{a}_{i} \text { false }} P\left(\neg a_{i} \mid c_{1}\right), \text { and } \\
& P\left(c_{2} \mid \bar{a}\right)=P\left(c_{2}\right) \prod_{\bar{a}_{i} \text { true }} P\left(a_{i} \mid c_{2}\right) \prod_{\bar{a}_{i} \text { false }} P\left(\neg a_{i} \mid c_{2}\right) .
\end{aligned}
$$


The predicted brain state may then be taken as the state $i$ for which $P\left(c_{i} \mid \bar{a}\right)$ is highest, or by setting a threshold on the log likelihood ratio

$$
\log \left(\frac{P\left(c_{1} \mid \bar{a}\right)}{P\left(c_{2} \mid \bar{a}\right)}\right) .
$$

When applying feature grouping, as outlined above, one applies the naive Bayesian calculation on features in the most stimulus-specific feature groups only.

\subsubsection{Scale estimation}

As mentioned in Section 6.1, the region-based method provides scale estimates for task-specific regions, independent of a statistical threshold. Below, we present the scale-estimation mechanism of the region-based method.

In the scale-estimation mechanism, we exploit information on the scales of the specific wavelets that together define a region as task-specific. The main idea is that wavelets of a scale that is close to the spatial extent of the region will, on average, have a higher specificity measure than wavelets of other scales. This idea will be translated into a statistical method that considers the distribution of wavelet scales. The scale-estimation mechanism consists of two steps. In the first step, a per-vertex scale estimate is obtained using the main idea expressed above. In the second step, scale estimates of neighbouring vertices are combined to arrive at a scale estimate for an entire cortical region.

\section{First step in scale estimation}

In the first step, our starting point is the weighted sum given in (6.6). The difference with vertex classification is that we do not weight for the wavelet sizes, but that we set the weights $w_{i, j}$ to +1 or to -1 , according to whether the wavelet part covering a vertex is positive or negative. In addition to calculating the weighted sum (6.6) over all wavelet scales, we also calculate it separately for each scale. If we define $O_{j, g, \sigma}$ as the subset of wavelets of scale $\sigma$ that overlap vertex $j$, and for which the log likelihood lies in the $g$ th largest positive group or the $g$ th largest negative group, then the per-scale estimate is calculated as:

$$
\begin{aligned}
L\left(v_{j}, G, \sigma\right) & =\sum_{g=1}^{G} \sum_{i \in O_{j, g, \sigma}} w_{i, j} \log \left(\frac{P\left(a_{i} \mid c_{1}\right)}{P\left(a_{i} \mid c_{2}\right)}\right) \\
& =\sum_{g=1}^{G} \sum_{i \in O_{j, g}, \sigma} \log \left\{\left(\frac{P\left(a_{i} \mid c_{1}\right)}{P\left(a_{i} \mid c_{2}\right)}\right)^{w_{i, j}}\right\} .
\end{aligned}
$$

The goal of the scale-estimation mechanism is to determine which scale $\sigma$ explains the specificity best. For this purpose, we do not care whether the specificity is for stimulus 1 or for stimulus 2. Specificity is indicated by both values of $L\left(v_{j}, G, \sigma\right)$ that are much larger than zero, and values of $L\left(v_{j}, G, \sigma\right)$ that are much 
smaller than zero. Hence, we may assume that the absolute value of $L\left(v_{j}, G, \sigma\right)$ is a measure of specificity. We may thus normalise over the scales, and determine the probability of a scale as specific:

$$
P\left(\sigma \mid L\left(v_{j}, G, \sigma\right)\right)=\frac{\left|L\left(v_{j}, G, \sigma\right)\right|}{\sum_{\sigma}\left|L\left(v_{j}, G, \sigma\right)\right|} .
$$

Since we know the scales used in the wavelet decomposition, we can calculate the expected value for the scale $E[\sigma]_{j}$ for each vertex $v_{j}$. The expected values for each vertex need to be incorporated into a scale estimate for an entire specific region.

\section{Second step in scale estimation}

In the second step, we therefore combine the per-vertex scale estimates of neighbouring vertices in order to obtain a region-wide scale estimate. Naively, we might try to average the scale estimate over the regions we know to be specific. However, in experiments with real data, we do not know these regions in advance. In addition, we do not know the region's extent in the first place; this is a problem that our method attempts to solve. We provide only the centre point of the actual region as input to the scale estimator. The scale estimator will calculate the region scale, by constructing circles of different radii around the centre point, and taking that radius for which the (weighted) per-vertex scale estimates within the circle matches the radius best. For a given radius $r$, the estimator takes into account all vertices that lie within radius $r$ of the centre point, and calculates a weighted average of $E[\sigma]_{j}$ (weighted by $\left|L\left(v_{j}, G\right)\right|$, that is) over those vertices. The scale for which this weighted average is closest to $r$ is then selected as the characteristic scale $\sigma_{R . j, G}$ of the region:

$$
\sigma_{R, j, G}=\arg \min _{r}\left|\frac{\iint_{\text {Area of radius } r}|L(v, G)| E[\sigma] \mathrm{d} A}{\iint_{\text {Area of radius } r}|L(v, G)| \mathrm{d} A}-r\right| .
$$

When using this method in practise, the neurocognitive researcher may interactively select a region centre.

This concludes our description of the region-based method. In the next section we describe the experimental set-up for evaluating the performance of the method.

\subsection{Experimental set-up}

In this section, we describe the experimental set-up used to evaluate the performance of the region-based method on simulated data. 


\subsubsection{Dependent and independent variables}

All experiments are performed on the cortex of the left brain hemisphere, where the background noise is actual fMRI data sampled from a subject at rest. In this experiment, the activations are simulated, because working with simulated activations allows us (1) to control the signal and noise conditions to evaluate their impact on the performance of the region-based method, and (2) to have a reference with respect to the regions that should be detected. The background noise is sampled using a 3-Tesla Siemens Allegra fMRI scanner. A total of 333 volumes were sampled at a TR of $1,500 \mathrm{~ms}$. Using the preprocessing steps of BrainVoyagerQX outlined in Subsection 6.2.2, the data is projected into the Talairach space, and then sampled on a cortical grid of 10,242 vertices. This results in a background-noise set of 10,242 vertices by 333 time points.

Activations in the brain are simulated using the gamma-function-based model by Boynton et al. (1996). An event-related paradigm is used for the activations, where 15 exposures of stimulus 1 are alternated with 15 exposures of stimulus 2 . We use two contrasting stimuli, of which the cortical layout and signal properties will be discussed below.

In our experiments, we employ three types of independent variables to assess the performance of the region-based method. The three types of independent variables are: (1) activation pattern, (2) signal and contrast conditions, and (3) method-specific choices. These types of variables will be described below. The two dependent variables in our experiment are (1) region-detection performance and (2) scale-estimation performance. They are defined in Subsection 6.5.2.

\section{Activation pattern}

The first type of independent variable is the simulated spatial pattern of activation. For our experiments we have to choose a brain layout of active and stimulusspecific regions; a pattern of activation. In all patterns of activation, there are three active areas. The first area has a stronger BOLD response to stimulus one than to stimulus two. The second area has a stronger BOLD response to stimulus two than to stimulus one. The third area responds with equal BOLD amplitude to both stimuli, and should thus not be detected as a specific region.

In order to determine whether the region-based method can produce a reliable scale estimate, we use two distinct patterns of activation that differ in the spatial extents of the active regions. In the first pattern of activation, the first and second areas are equally large (namely, 5 spherical-grid units); the third area is also 5 units in size. In the second pattern of activation, the first area is considerably smaller (3) than the second area (8); the third area has the same size as in the first pattern of activation (5).

Figure 6.5 illustrates the two patterns of activation. The top panel shows the brain having equally-sized regions of activation. The three dark regions respond to the stimuli. The top dark region responds equally to both stimuli. The middle dark region responds stronger to stimulus 1 than to stimulus 2. The bottom dark region responds stronger to stimulus 2 than to stimulus 1 . The bottom panel of 
the figure shows the brain having unequally-sized regions. The meaning of the dark regions is the same as in the top panel.

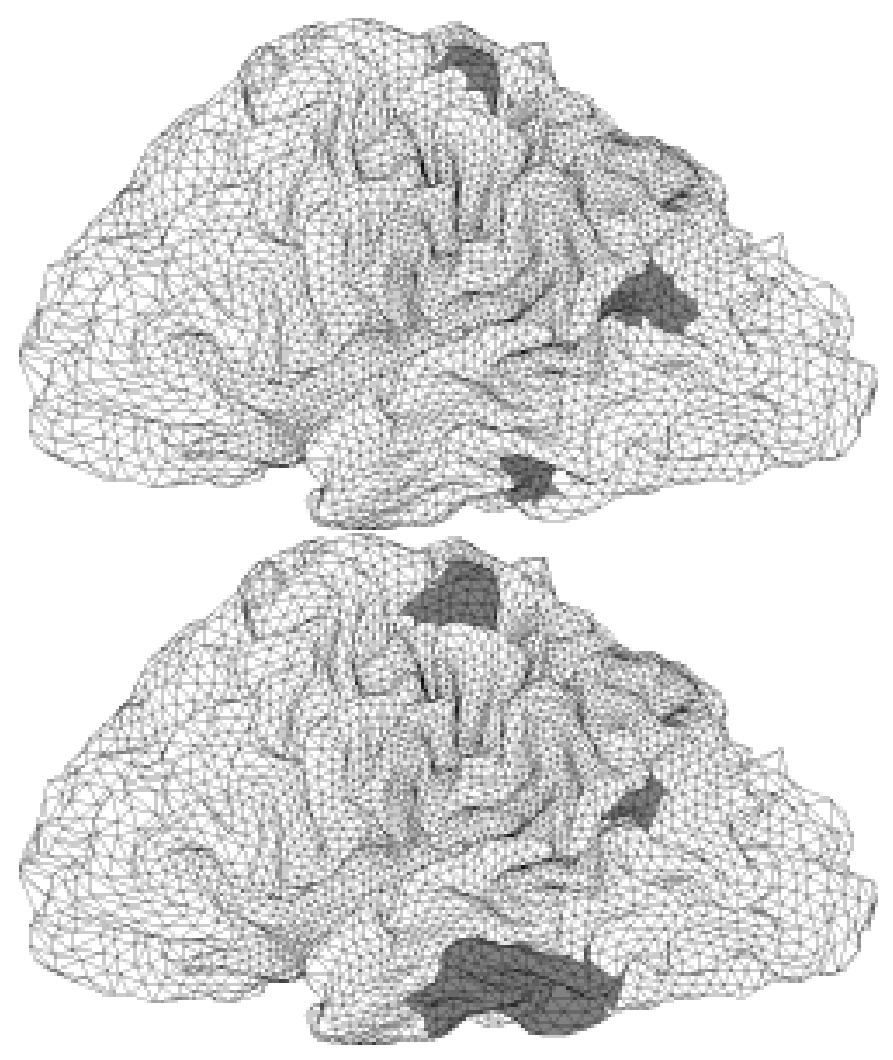

Figure 6.5: Two different patterns of activation. Dark shaded regions indicate activated regions. Top: brain with equally-sized regions of activation. Bottom: brain with unequally-sized regions of activation.

\section{Signal and contrast conditions}

The second type of independent variable involves the signal and contrast properties: the signal-to-noise amplitude ratio (SNAR) and the contrast-to-noise amplitude ratio (CNAR). We explain both ratios with the help of Figure 6.6.

The two curves in Figure 6.6 denote the average BOLD responses to stimulus 1 (lower curve) and to stimulus 2 (upper curve) over a certain active region. The grey area labelled "noise floor" denotes the standard deviation of the BOLD background noise in the active region. The SNAR and CNAR are defined relative to the noise floor as follows: 


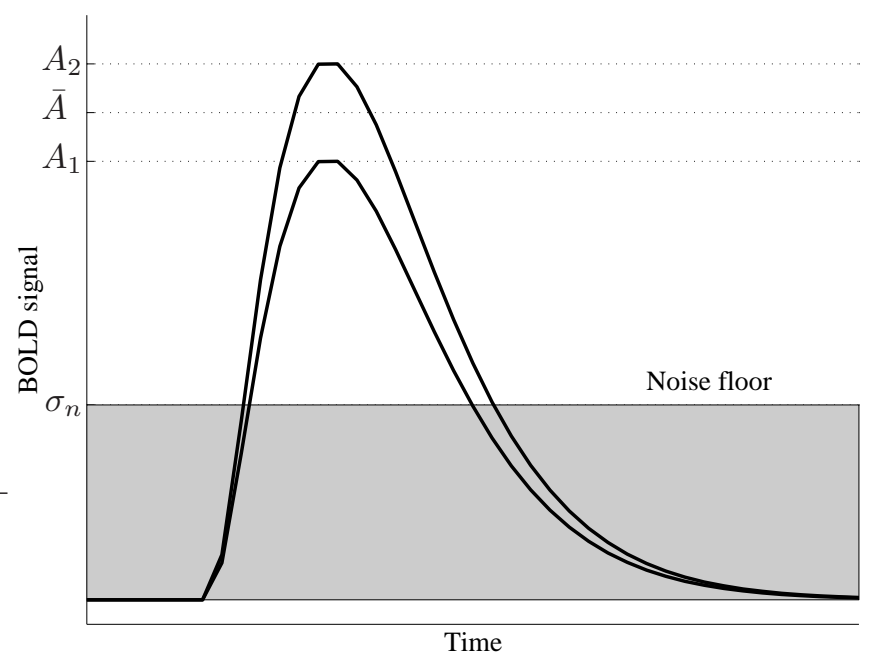

Figure 6.6: Signal-to-noise amplitude ratio and contrast-to-noise amplitude ratio.

$$
\begin{aligned}
\mathrm{SNAR} & \equiv \frac{\bar{A}}{\sigma_{n}}, \text { and } \\
\mathrm{CNAR} & \equiv \frac{\left|A_{1}-A_{2}\right|}{\sigma_{n}} .
\end{aligned}
$$

In this way, the ratios take into account the signal and contrast amplitude magnitude compared to the average noise amplitude. In our experiments the activations are simulated, which allows us to control the values of the SNAR and CNAR in order to determine the performance of our method under different ratios. We choose SNARs of 1.0, 1.5, 2.0, 2.5, 3.0, and 4.0, and we use CNARs of 0.1, $0.2,0.5,0.7,1.0,1.5$, and 2.0 . In this manner, we test a total of $6 \times 7=42$ signal and contrast conditions.

\section{Method-specific choices}

The third type of independent variable involves three method-specific choices. The first choice of the region-based method is the number and scales of wavelets used, as it is not known in advance which wavelet scales will work best. We can bound the wavelet scales based on both engineering and anatomical insights. First, we give the engineering insight; a wavelet cannot be smaller than a scale of 1 grid unit, and that is the smallest scale we will use. Second, we give the anatomical insight; in our brain representation, a scale of 16 grid units encompasses such a large cortical area, that areas specific to a stimulus are unlikely to be larger than that. Therefore, 16 grid units is the largest wavelet scale we will use. Hence, we 
will use dyadic divisions between 1 and 16 grid units, leading to six different scale grids.

The second choice concerns the types of wavelets to be used: just averaging wavelets, or both averaging and gradient wavelets. As stated in Subsection 6.4.1, the inclusion of gradient wavelets might yield a better detection, because the region's edges are captured. However, using gradient wavelets in addition to averaging wavelets increases the dimensionality of the feature space. Whether the capability to capture regions' edges outweighs the disadvantage of having a larger feature-space dimensionality is not clear a priori. Therefore, we evaluate (1) the use of averaging wavelets only, and (2) the use of both averaging and gradient wavelets.

The third choice is whether to correct for noise magnitude, in order to reduce the changes of selecting features that statistically seem discriminative, but this might be merely so because of noise effects. This topic will be covered in our discussion in Section 6.7

\subsubsection{Evaluation criteria}

The evaluation criteria assess the specific-region-detection performance, and the scale-estimation performance.

The specific-region-detection performance will be measured in relation to a "baseline" method: the commonly used vertex-based General Linear Model (Holmes et al., 1997). The GLM is tuned in such a way that the false-discovery rate (Genovese, Lazar, and Nichols, 2002) is 0.10. However, if a detection rate of 1.00 is achieved using the FDR threshold, the threshold is set to the lowest possible value at which the detection rate is still 1.00; this is done to prevent an excessive amount of false detections. The evaluation criterion for stimulus-specific activation detection (SSA) performance is the detection rate; at a given false-detection rate, a higher detection rate signifies a better method.

The scale-estimation performance will be measured in an absolute sense, because none of the currently existing methods provides a baseline scale-estimation result against which our results can be compared. For each specific region we determine the estimated scale using equation (6.12), and compare this scale to the known scale of the region. The dependent variable for measuring the scaleestimation performance is the absolute difference between the estimated and actual region scales; a lower absolute difference signifies a better result.

\subsection{Results}

In this section, we present the results obtained with the region-based method on the simulated activity data. The results will be presented in two parts, reflecting the fact that we have two types of performance criteria. First, we present the specific-region-detection performance in Subsection 6.6.1. Subsequently, we present the scale-estimation performance in Subsection 6.6.2. 


\subsubsection{Region-detection results}

To obtain a baseline performance to which we can compare our region-based performance, we measured the GLM performances under all experimental variations. The baseline results in terms of detection rates and false-positive rates for the GLM are displayed in the Tables 6.1 and 6.2 for the two activation patterns of Figure 6.5.

Table 6.1: Detection and false-positive results for the general linear model (GLM) on the brain with equally sized regions

\begin{tabular}{l|c|c|c|c|c|c}
\multirow{2}{*}{$C N A R$} & \multicolumn{7}{|c}{$S N A R$} \\
\cline { 2 - 7 } & 1.0 & 1.5 & 2.0 & 2.5 & 3.0 & 4.0 \\
\hline 0.1 & 0.0833 & 0.0833 & 0.0750 & 0.0667 & 0.0667 & 0.0667 \\
0.2 & 0.4417 & 0.4250 & 0.4417 & 0.4250 & 0.4167 & 0.4167 \\
0.5 & 1.0000 & 1.0000 & 1.0000 & 1.0000 & 1.0000 & 1.0000 \\
0.7 & 1.0000 & 1.0000 & 1.0000 & 1.0000 & 1.0000 & 1.0000 \\
1.0 & 1.0000 & 1.0000 & 1.0000 & 1.0000 & 1.0000 & 1.0000 \\
1.5 & 1.0000 & 1.0000 & 1.0000 & 1.0000 & 1.0000 & 1.0000 \\
2.0 & 1.0000 & 1.0000 & 1.0000 & 1.0000 & 1.0000 & 1.0000 \\
\hline & \multicolumn{7}{|c}{$S N A R$} \\
$C N A R$ & 1.0 & 1.5 & 2.0 & 2.5 & 3.0 & 4.0 \\
\cline { 2 - 7 } 0.1 & 0.1264 & 0.1263 & 0.1263 & 0.1264 & 0.1264 & 0.1264 \\
0.2 & 0.1264 & 0.1264 & 0.1264 & 0.1264 & 0.1264 & 0.1263 \\
0.5 & 0.1137 & 0.1369 & 0.0765 & 0.0862 & 0.0934 & 0.0832 \\
0.7 & 0.0086 & 0.0070 & 0.0111 & 0.0076 & 0.0083 & 0.0118 \\
1.0 & 0.0047 & 0.0047 & 0.0047 & 0.0047 & 0.0047 & 0.0047 \\
1.5 & 0.0047 & 0.0047 & 0.0047 & 0.0047 & 0.0047 & 0.0047 \\
2.0 & 0.0047 & 0.0047 & 0.0047 & 0.0047 & 0.0047 & 0.0047 \\
\hline
\end{tabular}

The top halve of Table 6.1 shows the detection rate under different signal and contrast conditions, and the lower halve of Table 6.1 shows the false-detection rate under the same conditions. The rows in each of the table halves denote the contrast-to-noise amplitude ratio (CNAR), and the columns of each of the table halves denote the signal-to-noise amplitude ratio. The layout of Table 6.2 is the same as the layout of Table 6.1.

In order to obtain results for the region-based method that are comparable to the GLM, the threshold for the region-based method is chosen in such a way that the false-positive rate is equal to that of the GLM. This is done for a 1feature group estimate (i.e., taking only the most salient wavelets into account), a 2-feature group estimate, etc, until all wavelets have been taken into account. The number of integrated feature groups for the final result is chosen in such a way that the detection rate is highest. 
Table 6.2: Detection and false-positive results for the general linear model on the brain with unequally sized regions

\begin{tabular}{l|c|c|c|c|c|c}
\multirow{2}{*}{ CNAR } & \multicolumn{7}{|c}{$S N A R$} \\
\cline { 2 - 7 } & 1.0 & 1.5 & 2.0 & 2.5 & 3.0 & 4.0 \\
\hline 0.1 & 0.0236 & 0.0236 & 0.0276 & 0.0276 & 0.0236 & 0.0276 \\
0.2 & 0.1693 & 0.1693 & 0.1417 & 0.1732 & 0.1732 & 0.1614 \\
0.5 & 0.9606 & 0.9528 & 0.9646 & 0.9606 & 0.9724 & 0.9528 \\
0.7 & 1.0000 & 1.0000 & 1.0000 & 1.0000 & 1.0000 & 1.0000 \\
1.0 & 1.0000 & 1.0000 & 1.0000 & 1.0000 & 1.0000 & 1.0000 \\
1.5 & 1.0000 & 1.0000 & 1.0000 & 1.0000 & 1.0000 & 1.0000 \\
2.0 & 1.0000 & 1.0000 & 1.0000 & 1.0000 & 1.0000 & 1.0000 \\
\hline & \multicolumn{7}{|c}{$S N A R$} \\
$C N A R$ & 1.0 & 1.5 & 2.0 & 2.5 & 3.0 & 4.0 \\
\hline 0.1 & 0.1271 & 0.1271 & 0.1270 & 0.1271 & 0.1271 & 0.1270 \\
0.2 & 0.1271 & 0.1271 & 0.1272 & 0.1272 & 0.1271 & 0.1272 \\
0.5 & 0.1271 & 0.1272 & 0.1272 & 0.1271 & 0.1271 & 0.1270 \\
0.7 & 0.0623 & 0.0647 & 0.0741 & 0.0924 & 0.0807 & 0.1243 \\
1.0 & 0.0096 & 0.0097 & 0.0097 & 0.0096 & 0.0096 & 0.0095 \\
1.5 & 0.0093 & 0.0093 & 0.0093 & 0.0093 & 0.0093 & 0.0093 \\
2.0 & 0.0093 & 0.0093 & 0.0093 & 0.0093 & 0.0093 & 0.0093 \\
\hline
\end{tabular}

\section{The results summarised}

Table 6.3 shows the summarised results for the region-based method applied to both patterns of activation. The table shows the dependent variable - the detection rate - for all different values of the independent variables. Table 6.3 consists of 6 block rows and 2 block columns, that represent the performances obtained for the 6 ranges of scales and the 2 patterns of activation. The block rows each correspond to a given range of wavelet scales used in the classification. The scales are indicated in the left column as $\sigma_{\min }-\sigma_{\max }$, on a dyadic grid. For instance, $4-16$ implies that wavelet scales 4,8 , and 16 have been included in the wavelet transform. Each of the block columns corresponds to one of the two patterns of activation. Each of the table's block elements is composed of 7 rows and 6 columns, where the rows correspond to different contrast-to-noise ratios (CNARs), and the columns correspond to different signal-to-noise ratios (SNARs). Each entry in the table shows the dependent variable; the detection rate of our region-based method at the same false-positive rate as the GLM.

From the results, we may make two main observations. The first observation is that, for CNARs of 0.7 and higher, our method detects specific brain regions with a detection rate equal to, or only very slightly less, than the traditional GLM method. The fact that the specific regions are not always captured fully is likely attributable to the discrete nature of our wavelet features; a specific region will almost never be described well by one single wavelet. Rather, its outlines are determined by a number of wavelets overlapping the region. 
Table 6.3: Detection-rate results for each of the two activation patterns, for several wavelet scales, and for all combinations of SNARs and CNARs.

\begin{tabular}{|c|c|c|c|c|c|c|c|c|c|c|c|c|c|}
\hline \multirow[b]{3}{*}{ Scales } & \multirow[b]{3}{*}{ CNAR } & \multicolumn{6}{|c|}{ Brain with unequally-sized regions } & \multicolumn{6}{|c|}{ Brain with equally-sized regions } \\
\hline & & \multicolumn{6}{|c|}{ SNAR } & \multicolumn{6}{|c|}{ SNAR } \\
\hline & & 1.0 & 1.5 & 2.0 & 2.5 & 3.0 & 4.0 & 1.0 & 1.5 & 2.0 & 2.5 & 3.0 & 4.0 \\
\hline \multirow[t]{7}{*}{$1-4$} & 0.1 & 0.055 & 0.063 & 0.055 & 0.063 & 0.059 & 0.043 & 0.033 & 0.050 & 0.042 & 0.033 & 0.033 & 0.042 \\
\hline & 0.2 & 0.224 & 0.220 & 0.240 & 0.213 & 0.209 & 0.220 & 0.383 & 0.383 & 0.408 & 0.383 & 0.392 & 0.375 \\
\hline & 0.5 & 0.972 & 0.957 & 0.969 & 0.965 & 0.969 & 0.972 & 0.967 & 0.975 & 0.958 & 0.967 & 0.967 & 0.975 \\
\hline & 0.7 & 0.992 & 0.992 & 0.996 & 0.996 & 0.992 & 0.996 & 0.983 & 0.983 & 0.992 & 0.983 & 0.992 & 0.975 \\
\hline & 1.0 & 0.996 & 1.000 & 1.000 & 1.000 & 0.992 & 0.996 & 0.992 & 0.992 & 0.992 & 0.992 & 0.992 & 0.983 \\
\hline & 1.5 & 1.000 & 1.000 & 1.000 & 1.000 & 1.000 & 1.000 & 1.000 & 1.000 & 1.000 & 1.000 & 1.000 & 1.000 \\
\hline & 2.0 & 1.000 & 1.000 & 1.000 & 1.000 & 1.000 & 1.000 & 1.000 & 1.000 & 1.000 & 1.000 & 1.000 & 1.000 \\
\hline \multirow[t]{7}{*}{$1-8$} & 0.1 & 0.008 & 0.004 & 0.008 & 0.008 & 0.004 & 0.004 & 0.017 & $\begin{array}{l}0.017 \\
\end{array}$ & 0.017 & 0.025 & 0.017 & 0.017 \\
\hline & 0.2 & 0.323 & 0.315 & 0.315 & 0.327 & 0.323 & 0.335 & 0.442 & 0.458 & 0.475 & 0.458 & 0.467 & 0.508 \\
\hline & 0.5 & 0.965 & 0.961 & 0.965 & 0.961 & 0.972 & 0.965 & 0.992 & 0.992 & 0.975 & 0.975 & 0.983 & 0.983 \\
\hline & 0.7 & 0.992 & 0.988 & 0.996 & 1.000 & 1.000 & 1.000 & 0.992 & 0.992 & 0.992 & 0.992 & 0.992 & 0.992 \\
\hline & 1.0 & 1.000 & 1.000 & 1.000 & 1.000 & 1.000 & 1.000 & 1.000 & 1.000 & 1.000 & 1.000 & 1.000 & 1.000 \\
\hline & 1.5 & 1.000 & 1.000 & 1.000 & 1.000 & 1.000 & 1.000 & 1.000 & 1.000 & 1.000 & 1.000 & 1.000 & 1.000 \\
\hline & 2.0 & 1.000 & 1.000 & 1.000 & 1.000 & 1.000 & 1.000 & 1.000 & 1.000 & 1.000 & 1.000 & 1.000 & 1.000 \\
\hline \multirow[t]{7}{*}{$2-8$} & 0.1 & 0.000 & 0.000 & 0.000 & 0.000 & 0.000 & 0.000 & 0.000 & 0.000 & 0.000 & 0.000 & 0.000 & 0.000 \\
\hline & 0.2 & 0.382 & 0.386 & 0.382 & 0.382 & 0.390 & 0.386 & 0.575 & 0.567 & 0.575 & 0.558 & 0.583 & 0.575 \\
\hline & 0.5 & 0.972 & 0.972 & 0.969 & 0.969 & 0.969 & 0.969 & 0.983 & 0.983 & 0.975 & 0.983 & 0.983 & 0.983 \\
\hline & 0.7 & 0.992 & 0.988 & 0.992 & 0.992 & 0.992 & 0.996 & 1.000 & 0.983 & 1.000 & 0.992 & 1.000 & 0.992 \\
\hline & 1.0 & 0.996 & 0.996 & 0.996 & 0.996 & 0.996 & 0.996 & 1.000 & 1.000 & 1.000 & 1.000 & 1.000 & 1.000 \\
\hline & 1.5 & 1.000 & 1.000 & 1.000 & 1.000 & 1.000 & 1.000 & 1.000 & 1.000 & 1.000 & 1.000 & 1.000 & 1.000 \\
\hline & 2.0 & 1.000 & 1.000 & 1.000 & 1.000 & 1.000 & 1.000 & 1.000 & 1.000 & 1.000 & 1.000 & 1.000 & 1.000 \\
\hline \multirow[t]{7}{*}{$2-16$} & 0.1 & \begin{tabular}{l|l}
0.000 \\
\end{tabular} & 0.000 & 0.000 & $\begin{array}{l}0.000 \\
\end{array}$ & 0.000 & 0.000 & 0.000 & \begin{tabular}{l|l}
0.000 \\
\end{tabular} & 0.000 & \begin{tabular}{l|}
0.000 \\
\end{tabular} & 0.000 & 0.000 \\
\hline & 0.2 & 0.402 & 0.409 & 0.437 & 0.433 & 0.413 & 0.409 & 0.558 & 0.550 & 0.575 & 0.550 & 0.558 & 0.575 \\
\hline & 0.5 & 0.980 & 0.972 & 0.976 & 0.980 & 0.980 & 0.980 & 0.983 & 0.983 & 0.983 & 0.983 & 0.983 & 0.983 \\
\hline & 0.7 & 0.996 & 0.996 & 0.996 & 0.996 & 1.000 & 0.996 & 1.000 & 0.983 & 1.000 & 0.975 & 0.992 & 0.992 \\
\hline & 1.0 & 0.996 & 1.000 & 0.996 & 1.000 & 1.000 & 1.000 & 1.000 & 1.000 & 1.000 & 1.000 & 1.000 & 1.000 \\
\hline & 1.5 & 1.000 & 1.000 & 1.000 & 1.000 & 1.000 & 1.000 & 1.000 & 1.000 & 1.000 & 1.000 & 1.000 & 1.000 \\
\hline & 2.0 & 1.000 & 1.000 & 1.000 & 1.000 & 1.000 & 1.000 & 1.000 & 1.000 & 1.000 & 1.000 & 1.000 & 1.000 \\
\hline \multirow[t]{7}{*}{$4-16$} & 0.1 & 0.000 & 0.000 & 0.000 & 0.000 & 0.000 & 0.000 & 0.000 & 0.000 & 0.000 & 0.000 & 0.000 & 0.000 \\
\hline & 0.2 & 0.445 & 0.433 & 0.441 & 0.441 & 0.449 & 0.449 & 0.592 & 0.583 & 0.600 & 0.575 & 0.592 & 0.608 \\
\hline & 0.5 & 0.972 & 0.972 & 0.972 & 0.972 & 0.972 & 0.972 & 1.000 & 1.000 & 0.992 & 1.000 & 1.000 & 1.000 \\
\hline & 0.7 & 0.988 & 0.988 & 0.988 & 0.992 & 0.992 & 0.996 & 0.967 & 0.958 & 0.975 & 0.950 & 0.958 & 0.983 \\
\hline & 1.0 & 0.988 & 0.988 & 0.988 & 0.988 & 0.988 & 0.988 & 1.000 & 1.000 & 1.000 & 1.000 & 1.000 & 1.000 \\
\hline & 1.5 & 1.000 & 1.000 & 1.000 & 1.000 & 1.000 & 1.000 & 1.000 & 1.000 & 1.000 & 1.000 & 1.000 & 1.000 \\
\hline & 2.0 & 1.000 & 1.000 & 1.000 & 1.000 & 1.000 & 1.000 & 1.000 & 1.000 & 1.000 & 1.000 & 1.000 & 1.000 \\
\hline \multirow[t]{7}{*}{$8-16$} & 0.1 & 0.000 & 0.000 & 0.000 & 0.000 & 0.000 & 0.000 & 0.000 & 0.000 & 0.000 & 0.000 & 0.000 & 0.000 \\
\hline & 0.2 & 0.476 & 0.476 & 0.476 & 0.480 & 0.476 & 0.476 & 0.450 & 0.450 & 0.458 & 0.450 & 0.450 & 0.450 \\
\hline & 0.5 & 0.996 & 0.996 & 0.996 & 0.996 & 0.996 & 0.996 & 1.000 & 1.000 & 0.983 & 0.983 & 0.983 & 0.983 \\
\hline & 0.7 & 1.000 & 1.000 & 1.000 & 1.000 & 1.000 & 1.000 & 0.983 & 0.975 & 0.983 & 0.975 & 0.975 & 0.983 \\
\hline & 1.0 & 0.965 & 0.996 & 0.996 & 0.996 & 0.988 & 0.996 & 1.000 & 1.000 & 1.000 & 1.000 & 1.000 & 1.000 \\
\hline & 1.5 & 1.000 & 1.000 & 1.000 & 1.000 & 1.000 & 1.000 & 1.000 & 1.000 & 1.000 & 1.000 & 1.000 & 1.000 \\
\hline & 2.0 & 1.000 & 1.000 & 1.000 & 1.000 & 1.000 & 1.000 & 1.000 & 1.000 & 1.000 & 1.000 & 1.000 & 1.000 \\
\hline
\end{tabular}

The following printing styles have been used as a visual indication of the quality of our results.

Bold: Our region-detection result is equal to or better than the GLM result.

Italics: Our region-detection result is almost as good as the GLM result (i.e., our detection rate is less than 2 per cent lower than the GLM's detection rate).

Normal: Our region-detection result is more than 2 per cent worse than the GLM. 
The second observation is that for CNARs of 0.2 , our method outperforms the GLM for almost all parameter settings and for both patterns of activation. In addition, for the pattern of activation with unequally sized regions, our method outperforms the GLM for almost all settings with a CNAR of 0.5 or less. Detecting any specific region under such a low CNAR at all is surprising enough in itself. Our features, having a spatial extent larger than one vertex, average the temporal noise over multiple vertices, and thus obtain - per feature - a higher CNAR, and hence achieve a better detection. While one might argue that the same result might be obtained using a GLM with extensive spatial smoothing of the BOLD response, our method is superior in terms of smoothing, because it automatically selects a smoothing scale appropriate for each specific region.

To show how the region-based method trades off the detection rate with the false-positive rate, we plot a Receiver Operating Characteristic in Figure 6.7. The figure shows the trade-off between the detection rate and the false-positive rate under a regime in which our method outperforms the GLM: the brain with unequally sized regions, wavelet scales $2-16$, a SNAR of 2.0, and a CNAR of 0.5. The figure shows that, for detection rates above about $73 \%$, our method clearly yields a more accurate region detection than the GLM.

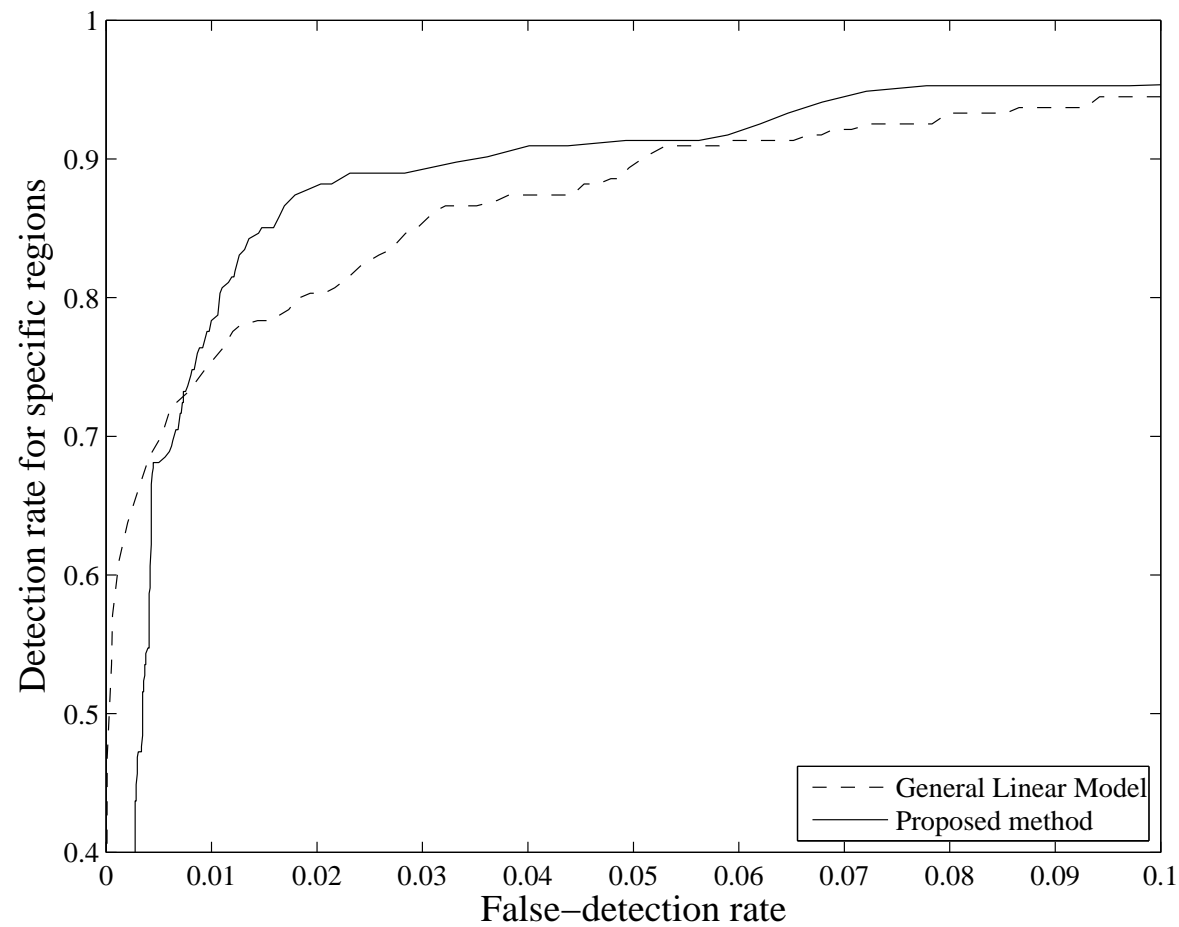

Figure 6.7: Receiver Operating Characteristic for the brain with unequally sized regions, wavelet scales $2-16$, a SNAR of 2.0, and a CNAR of 0.5. 


\subsubsection{Scale-estimation results}

The scale-estimation results of the region scale-estimation method are shown in Table 6.4. The interpretations of the (block) rows and (block) columns are the same as those in Table 6.3. The entries show the scale estimated for specific region 1 , and for specific region 2, at the same number of feature groups as in Table 6.3. That is, the scale estimate was obtained at that particular setting for which the detection rate is optimal. The following printing styles have been used as a visual indication of the quality of our results.

Bold: The estimated scale lies within 2 grid units of the actual scale.

Italics: The estimated scale lies within 3 grid units of the actual scale.

Normal: The estimated scale differs more than 3 grid units from the actual scale.

Here, a grid unit is defined in terms of the spherical grid introduced in Subsection 6.3.2. From these results, we may make three observations. The first observation is that scale estimates are generally better for the brain with unequally sized regions, than for the brain with equally sized regions. We postulate that this is due to grid of wavelet scales used. For the brain with unequally sized regions, the typical scales of the regions activated by the stimulus are either equal to a wavelet scale (8), or very close to two adjacent scales $(3$, in between scales 2 and 4 ). In the brain with equally sized regions, the scales of the regions activated by the stimulus are 5 which, while close to wavelet scale 4 , is very different from the next closest wavelet scale (8).

The second observation is that the quality of the scale estimates is strongly dependent on the wavelet scales used. This is not surprising, and can be explained best in terms of the brain with unequally sized regions. Whenever the largest wavelet scale is below 8, the scale of the largest region (8) cannot be reliably estimated; the estimated scale for a region always lies on the interval $\left[\sigma_{\min }, \sigma_{\max }\right]$, where $\sigma_{\min }$ is the smallest wavelet scale used, and $\sigma_{\max }$ is the largest wavelet scale used. Whenever the smallest wavelet scale is above 3 , the scale for the smallest specific region cannot be reliably estimated for the same reason. Thus, it stands to reason that the only experiments for which we may expect reliable scale estimates, are $2-8$ and $2-16$. Indeed, we observe that the estimated scales are closest to the actual region scales under those two conditions.

Third, we observe a similar dependence of quality on the CNAR as in the region-detection results. The best results are obtained for CNARs of 0.5 and higher. This is to be expected, since the noise level at lower CNARs causes very hard conditions for estimation. As in the region-detection results, the dependence on SNAR is smaller than the dependence on CNAR. This too, is to be expected, since we look at differences between responses only, and not at differences between responses and the baseline.

\section{Optimising for scale}

Although the scale estimates obtained in the results section are good, those scale estimates were obtained at the number of feature groups for which the detection 
Table 6.4: Region-scale estimation results for each of the two patterns of activation, for several wavelet scales, and for all combinations of SNARs and CNARs.

\begin{tabular}{|c|c|c|c|c|c|c|}
\hline $\begin{array}{l}\infty \\
\vdots \\
\omega\end{array}$ & $\begin{array}{l}+ \\
1 \\
5 \\
\end{array}$ & $\begin{array}{l}N \\
1 \\
5\end{array}$ & $\begin{array}{l}N \\
\infty\end{array}$ & $\vec{\infty}$ & 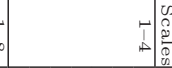 & \\
\hline 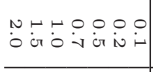 & 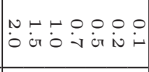 & 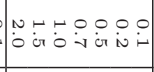 & 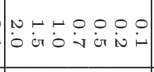 & 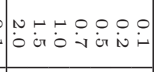 & 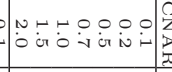 & \\
\hline 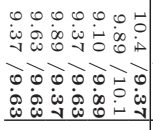 & 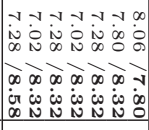 & 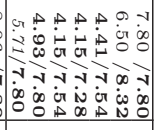 & 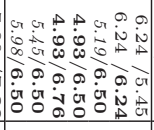 & 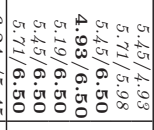 & 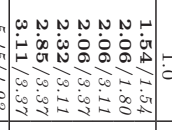 & \\
\hline 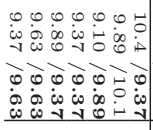 & 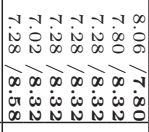 & 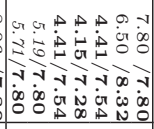 & 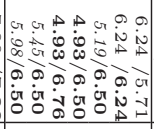 & 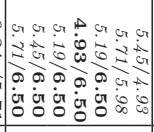 & 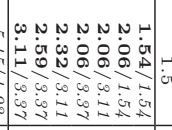 & ن. \\
\hline 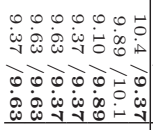 & 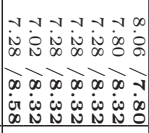 & 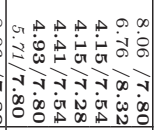 & 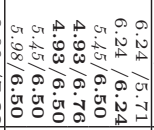 & 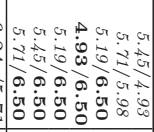 & 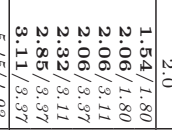 & م. \\
\hline 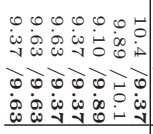 & 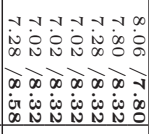 & 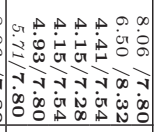 & 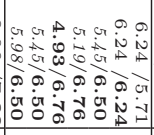 & 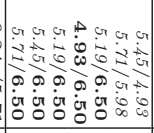 & 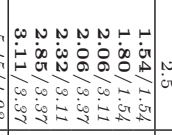 & ט. \\
\hline 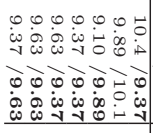 & 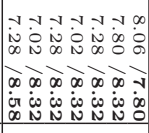 & 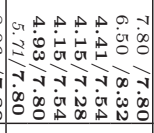 & 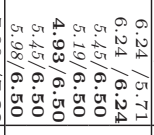 & 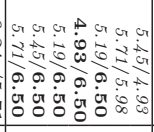 & 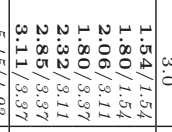 & 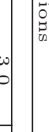 \\
\hline 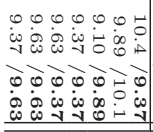 & 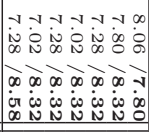 & 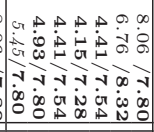 & 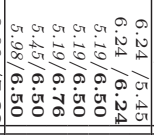 & 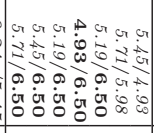 & 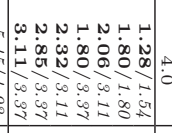 & \\
\hline 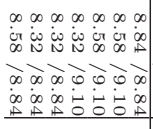 & 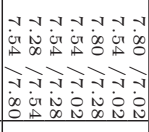 & 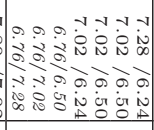 & 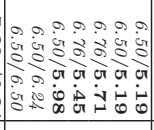 & 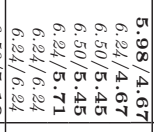 & 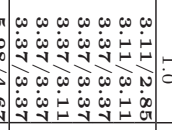 & \\
\hline 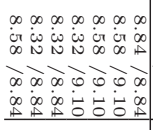 & 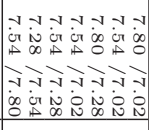 & 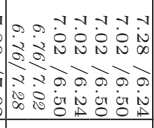 & 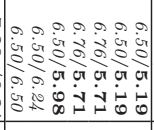 & 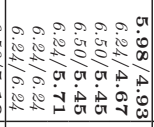 & 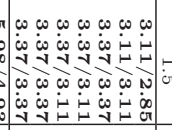 & t్ర \\
\hline 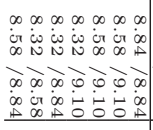 & 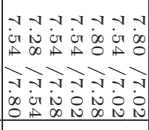 & 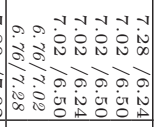 & 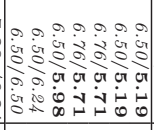 & 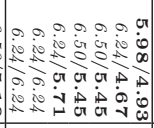 & 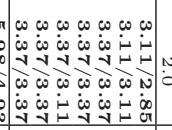 & 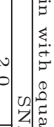 \\
\hline 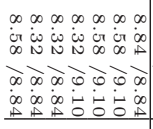 & 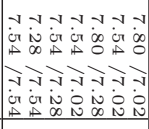 & 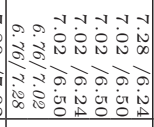 & 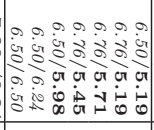 & 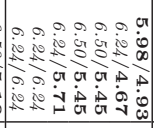 & 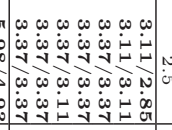 & 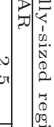 \\
\hline 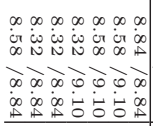 & 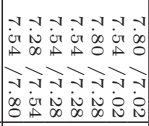 & 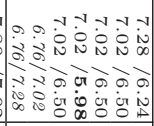 & 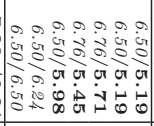 & 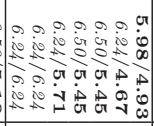 & 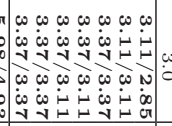 & $y$ \\
\hline 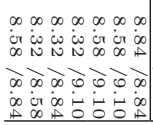 & 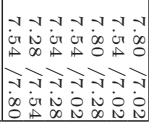 & 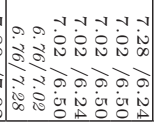 & 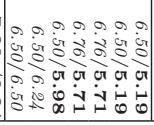 & 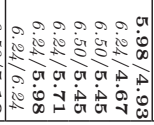 & 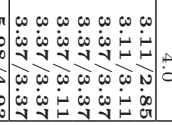 & \\
\hline
\end{tabular}


rate is highest. The number of feature groups for which the scale estimation is best might be different from the number of feature groups for which the detection rate is highest. Therefore, we would like to determine how good the scale estimates can be by themselves. In other words, we would like to determine for which number of feature groups the scale estimates are best, and to what degree this number differs from the number of feature groups for the best detection rate.

Figure 6.8 shows the scale estimate mismatch as a function of the feature fraction, and thus of the number of feature groups used in classification. This representative example is obtained for wavelet scales $2-16$, for a SNAR of 2.0, and for CNARs ranging from 0.5 to 2.0. The figure clearly shows that a feature fraction in the order of a few per cents is optimal for scale estimation. This is to be expected. When using too few features, one does not capture sufficient information to classify a region, and a region's scale, correctly. However, using too many features incorporates noise, given the very few instances that are available.

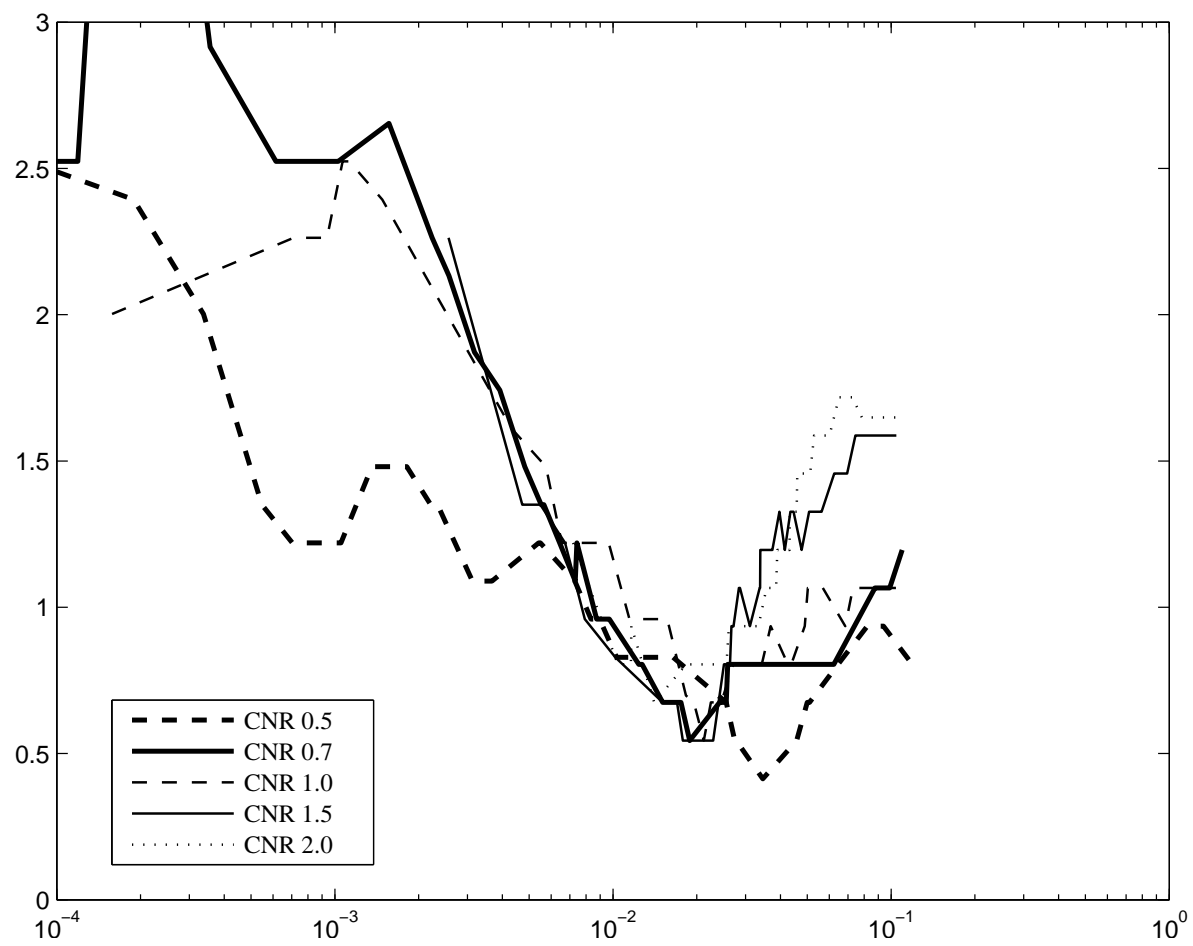

Figure 6.8: Scale estimate mismatch as a function of the number of features taken into account.

Table 6.5 contains the scale-estimation results for all experimental conditions, obtained at the number of feature groups for which the scale estimate is best.

It should be noted that, within a given combination of pattern of activation and wavelet scales (i.e., one $6 \times 7$ block-element in the Table), the same number of feature groups are used for all SNARs and CNARs. The optimal number of 
Table 6.5: Region-scale estimation results for each of the two patterns of activation, for several wavelet scales, and for all combinations of SNARs and CNARs - Optimised for scale.

\begin{tabular}{|c|c|c|c|c|c|c|}
\hline $\begin{array}{l}\infty \\
\stackrel{a}{\sigma} \\
\end{array}$ & $\stackrel{\Delta}{\Delta}$ & $\begin{array}{l}0 \\
\stackrel{1}{6} \\
\end{array}$ & $\begin{array}{l}N \\
\infty\end{array}$ & $\begin{array}{c}\tilde{\omega} \\
\infty\end{array}$ & 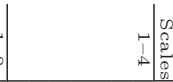 & \\
\hline 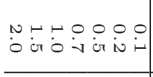 & 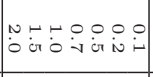 & 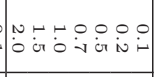 & 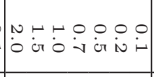 & 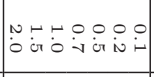 & 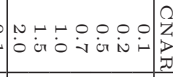 & \\
\hline 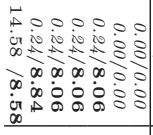 & 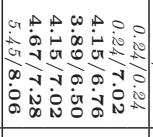 & 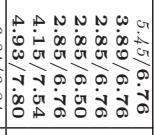 & 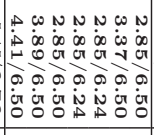 & 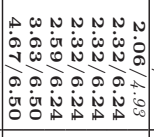 & 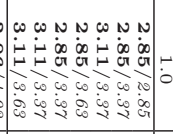 & \\
\hline 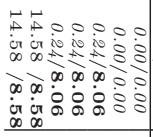 & 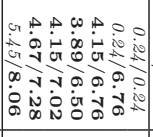 & 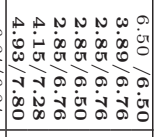 & 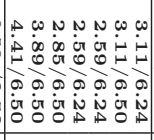 & 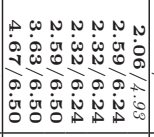 & 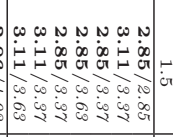 & $\underset{\sigma}{\mathscr{\sigma}}$ \\
\hline 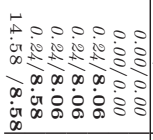 & 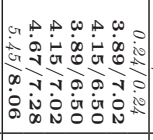 & 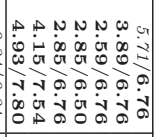 & 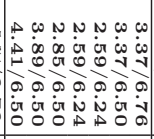 & 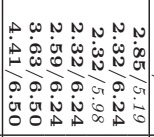 & O & 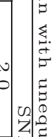 \\
\hline 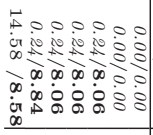 & 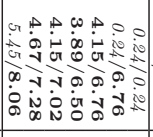 & 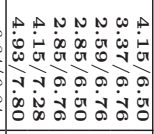 & 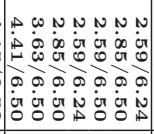 & 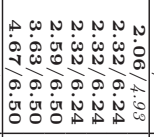 & r. & 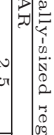 \\
\hline 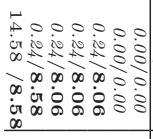 & 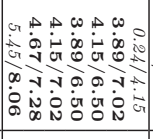 & 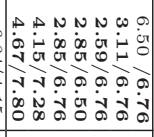 & 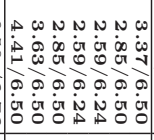 & 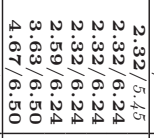 & 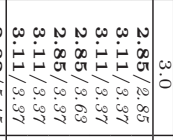 & 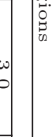 \\
\hline 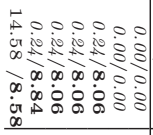 & 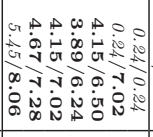 & 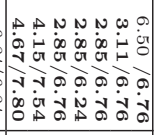 & 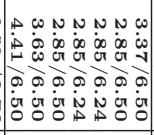 & 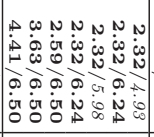 & 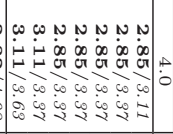 & \\
\hline 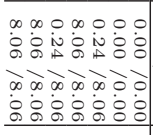 & 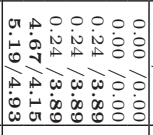 & 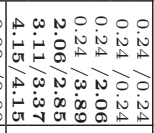 & 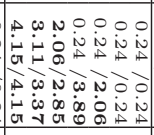 & 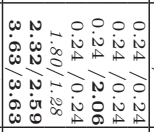 & 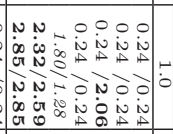 & \\
\hline 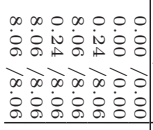 & 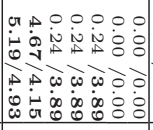 & 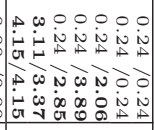 & 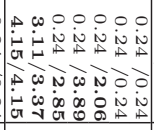 & 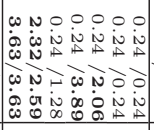 & 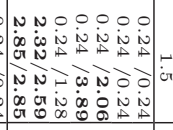 & 它 \\
\hline 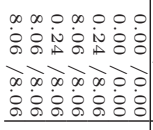 & 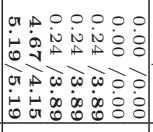 & 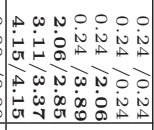 & 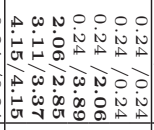 & 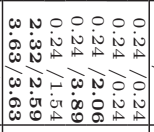 & 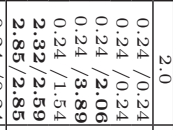 & 0 \\
\hline 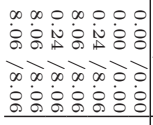 & 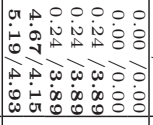 & 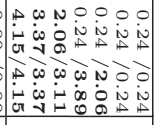 & 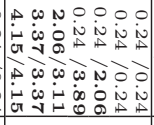 & 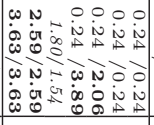 & 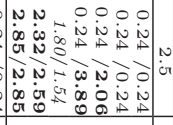 & 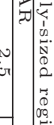 \\
\hline 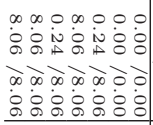 & 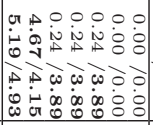 & 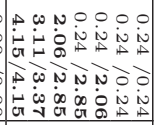 & 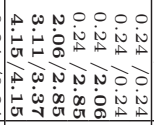 & 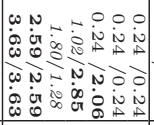 & 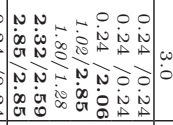 & $10^{\circ}$ \\
\hline 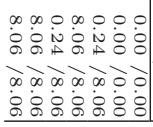 & 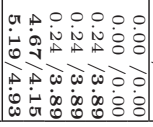 & 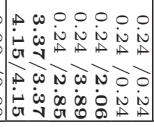 & 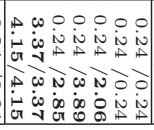 & 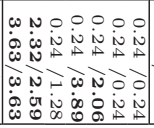 & 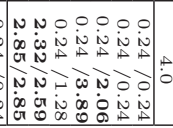 & \\
\hline
\end{tabular}


groups is that number for which the sum of squared scale mismatches across both specific regions and across all 42 SNAR/CNAR conditions is smallest.

There is one thing that should be noted about the results. Optimising for the scale is something that cannot be done in an actual experiment, since once does not know the scale. Therefore, the results presented in Table 6.5 should be regarded as the best possible scale estimates, which would have been obtained if we were able to select the right number of feature groups based on an external criterion.

\subsection{Challenging the use of simulated data}

The results obtained in the previous section are encouraging, with respect to the research questions RQ3 and RQ4; our method yields a stimulus-specific regiondetection performance comparable to, or better than, the GLM, and yields a reliable scale estimate. However, these findings are to be regarded in light of the fact that we used simulated fMRI data rather than real data. In this section, we discuss three consequences of the use of simulated data: consequences related to (1) feature selection, (2) scale-estimation performance, and (3) signal properties. A discussion of a more general nature will be provided later, in Chapter 8 .

The first consequence is related to the method we employed to determine the optimum number of feature groups for vertex classification. In this chapter, we chose that particular number of feature groups for which the detection rate - at the same false-positive rate as the GLM - is highest. This choice provided detailed information about the detection performance of the region-based method under various parameters. However, this choice of feature groups is possible only for simulated data; this choice required knowledge on precisely which cortical areas are stimulus specific. This knowledge is not available in real fMRI data, and another method to choose the number of feature groups will thus have to be used.

The second consequence is related to the scale-estimation performance, and has a course of reasoning similar to that of the first consequence. The results show that if a different feature-group selection method is used, scale-estimation performance might be improved further. However, in order to do so, information is required that is not available in real fMRI data.

The third consequence is related to the properties of simulated data. Although we have attempted to choose SNARs and CNARs that are representative for real fMRI activations, we do not know whether these choices are indeed valid. Furthermore, even though the model used to simulate activations (Boynton et al., 1996) is good, differences between the characteristics of simulated and real activations can have a significant effect on experimental results (Chen and Strother, 2006).

Given these consequences, we expect that an experiment on real fMRI data will yield a clearer assessment of the region-based method's performance and applicability than the ones provided in this chapter. 


\subsection{Chapter conclusions}

In this section we answer the research questions RQ3, RQ4, and RQ4a stated at the beginning of this chapter based on the insights obtained. In addition, we touch upon a broader range of applications for the region-based method.

Research question RQ3 reads:

Research question 3 (RQ3): Can a new region-based method detect brain regions with high specificity on a level comparable to or better than the standard method used in the field?

In order to answer this question, we compared our method against the general linear model (GLM) in an extensive range of experimental conditions. From the results, we may conclude that our method performs comparably for higher contrastto-noise ratios (CNARs), and clearly outperforms the GLM for low CNARs in the range 0.2 to 0.5 . We explain the superior performance of our method for lower CNARs by the fact that our method effectively reduces the noise through region-specific spatial smoothing.

Research second question RQ4 reads:

Research question 4 (RQ4): Can a new method deliver a reliable scale estimate for specific regions?

From our results, we may conclude that our method is capable of estimating the scale of specifically activated regions, although the selection of a proper fraction of features, and the selection of a specific region's exact location, remain open problems. Additional experiments revealed that noise weighting improves scale estimation in addition to improving specific-region detection.

Research question RQ4a reads:

Research question 4a (RQ4a): Can a new method effectively deal with classification in a very high-dimensional feature space?

This question is relevant, since the number of possible overlapping wavelets at multiple scales (yielding features in our method) is much larger than the number of vertices (yielding features for traditional methods). In other words, our preprocessing with wavelets, increases, rather than decreases, the dimensionality of the classification task. We observed that it is beneficial to select a subset of features for classification. We chose a very simple probability-based selection of features, but whereas more advanced selection methods may be available in related work, our selection method yields good results and has a low computational complexity.

The method we developed in this chapter is unique in the sense that it (1) is region based, (2) incorporates an inherent scale-estimation framework, and (3) requires no BOLD-specific information other than the experimental protocol. The fact that it is region based yields possibilities for a broader range of applications. For example, even though data measured from multiple test subjects may be very well aligned using contemporary algorithms, there always are small anatomical differences between subjects that cannot be corrected for in this manner. Our method, being based on averages and gradients computed over regions, is expected 
to be better able to accommodate such differences than traditional univariate methods such as the GLM. In the next chapter, we will put this expectation to test. 


\section{Chapter 7}

\section{Applying the region-based method to real data}

In the previous chapter, we presented the region-based method and applied it successfully to simulated fMRI data. In this chapter, we put our method to the test on real data.

The application of the region-based method to real multi-subject fMRI data poses two problems. The first problem is that our method is tuned to the simulated data. Although a fairly reliable model for simulation was used (Boynton et al., 1996), the differences between simulated and real fMRI data can be substantial (Chen and Strother, 2006). The second problem is that, due to anatomical differences between subjects, the locations of specific regions may differ across subjects. Since we would like to use data from multiple subjects, we have to find a solution.

In this chapter, we pose two research questions addressing both problems. These are research questions RQ5 and RQ6. Research question RQ5 reads:

Research question 5 (RQ5): Does the applicability of the new method generalise from simulated activation data to real data?

Research question RQ6 reads:

Research question 6 (RQ6): Can the region-based method cope with interpersonal differences in anatomy in a better way than standard methods?

In order to answer research questions RQ5 and RQ6, the region-based method will be applied to real fMRI data obtained from multiple subjects. The fact that the data is obtained from real fMRI experiments will aid in answering research question RQ5. In order to answer research question RQ6, we will use the region-based method in combination with the cortical-alignment procedures of the BRAINVOYAGERQX ${ }^{1}$ analysis software.

\footnotetext{
${ }^{1}$ http://www. brainvoyager.com
} 
The outline of this chapter as follows. Section 7.1 describes the selection of a suitable fMRI dataset on which our method will be tested. In Section 7.2, we mention three issues arising when applying our method to real data, and the approach to these issues. In Section 7.3, we motivate our experiment-specific method parameter choices, and the performance criteria we use. In Section 7.4, we show the results obtained by using our method. Section 7.5 discusses the results, and in Section 7.6 we draw conclusions and answer research questions RQ5 and RQ6.

\subsection{Data selection and preprocessing}

This section describes the selection of a dataset suitable for answering the research questions RQ5 and RQ6, and the preprocessing steps specific to this dataset. In Subsection 7.1.1, we present the selection criteria to which a dataset should conform, and the fMRI dataset to which this selection led. In Subsection 7.1.2, we describe the selected dataset in more detail. In Subsection 7.1.3, we describe the data preprocessing steps required.

\subsubsection{Data selection}

Our selection of a suitable fMRI dataset is guided by three criteria which are directly derived from the research questions currently under investigation. Research question RQ5 leads to two selection criteria. The first selection criterion is that former research on the same experiment has yielded consensus, of reference information, on specific regions against which both the traditional method's result and our result can be compared. The second selection criterion is that the experiment is set up in such a way that regions of clearly different spatial size are excited in the brain, as this allows us to assess whether our method correctly determines the differences in scales of the stimulus-specific regions. Preferably, one or both of the stimuli should cause specific activation in multiple brain regions, as this allows us to assess the region-scale determination method qualitatively in a multilaterate manner; the relative scale estimates among all regions should be correct in a qualitative sense.

Research question RQ6 leads to a third selection criterion. This third selection criterion is that the dataset contains results from experiments involving multiple subjects.

Using these three selection criteria, we selected a dataset that contains results from fMRI experiments involving a conventional faces-objects discrimination task (Kanwisher, McDermott, and Chun, 1997). In the fMRI experiment, subjects are exposed to a visual stimulus that is either a human face, or a non-face, i.e., another type of object, in a block-design paradigm (Kwong et al., 1992).

The faces-objects dataset satisfies our three selection criteria. With regard to the first selection criterion, previous research (Haxby et al., 1996; Halgren et al., 1999; Haxby et al., 2001) has created a consensus about the brain regions that are specific to the two stimuli. The dataset meets the second selection criterion because previous work shows that the brain regions activated when discriminating 
between faces and objects have very different scales. Based purely on the first two selection criteria, one could choose either brain hemisphere (or both) as the dataset. However, we have chosen to apply our method to the left hemisphere. The main reason for this choice is that the left hemisphere has four specific regions of different sizes, whereas the right hemisphere has just two. The specific regions will be presented in more detail in Subsection 7.3.2. Finally, the third selection criterion is satisfied because the dataset includes information on four subjects. This allows for evaluating inter-subject generalisation performance of the regionbased method.

The faces-objects dataset satisfies all our selection criteria, but uses an protocol design that differs slightly from the event-related design used in the previous chapter. This difference in design requires a small adaptation of the way in which samples are selected in the region-based method. The nature of this adaptation will be specified in detail in Subsection 7.2.1.

\subsubsection{Dataset properties}

In this subsection, we provide detailed information on the faces-objects dataset that will be used in this chapter. The dataset consists of a number of "runs", each of which consists of data obtained from one test subject during the fMRI experiment.

In a run, a subject is exposed to four images of faces, and four images of objects, in a block-design paradigm. Stimuli are alternated, and there are resting periods between each stimulus exposure. Each stimulus exposure lasts for 24 TRs (36 seconds). Resting periods last for 8 TRs (12 seconds). A run lasts for 266 TRs (399 seconds, or 6 minutes and 39 seconds). There are four different subjects, for which a total of 10 runs have been recorded. The BOLD signal is recorded with a TR of 1,500 ms on a 3-Tesla Siemens Allegra fMRI scanner.

\subsubsection{Data preprocessing}

A dataset containing data from multiple test subjects may necessitate an additional step to the preprocessing stage described in Subsection 6.2.2. In this additional step an alignment is performed to make a coarse correction for inter-subject anatomical differences. This correction is implemented in BRAINVOYAGERQX, and is performed as follows. The starting point for the extra preprocessing is the inflation of both brain hemispheres to spheres, as described in Subsection 6.2.2. In the extra preprocessing, an alignment is performed separately on the left and right hemispheres of all subjects. The alignment per hemisphere is performed by deforming the cortical spheres such that a suitable cost function - based on both surface stress and differences in surface curvature - is minimised. The mapping results in inter-subject average left and right hemispheres, and a so-called sphere-to-sphere mapping (SSM) that translates vertex-numbers from the subject's individual cortices to the average cortex, and back. In this chapter, we will use the fMRI signal of the subjects' left hemisphere, as shown in Figure 7.1. 


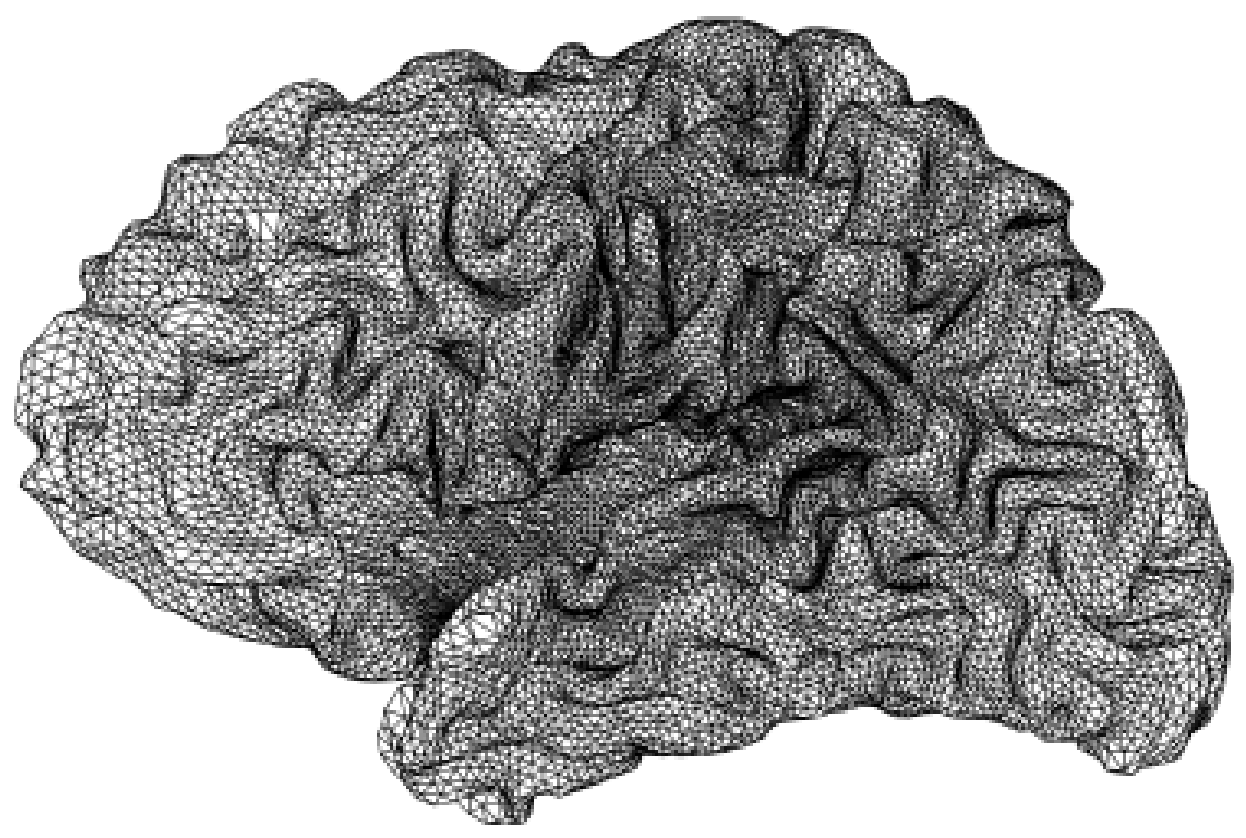

Figure 7.1: Left brain hemisphere mesh for one of the four subjects.

In our experiments, we will evaluate the performance of the region-based method with and without the SSM correction.

\subsection{Application of the region-based method to the dataset}

The application of our method to the faces-objects dataset requires three small modifications. The first modification is that, because the dataset is based on a block-design experiment, the extraction of sampling has to be carried out in a slightly different manner than in the previous chapter. The adapted sampling is described in Section 7.2.1. Second, the fact that we use real data rather than simulated data, has ramifications for our feature selection that will be described in Section 7.2.2. Third, the particular layout of task-specific regions in the dataset necessitates a small modification to our region-scale estimation method. This modification is described in Section 7.2.3.

\subsubsection{Sampling}

The faces-objects experiment is based on a block-design paradigm (Kwong et al., 1992). This means that the subject is exposed to a stimulus for a rather long period of time (24 TRs). The long duration per exposure also implies that only a few exposures take place within a given experimental run. Using the exact same 
feature-extraction model as in the previous chapter, would yield too few samples to train a classifier. However, we can exploit the fact that the long duration of the stimulus exposure enables the measurement of the BOLD step response, rather than the impulse response that is measured in an event-related paradigm. Therefore, we modify the sample-extraction procedure by extracting a sample for each TR in the plateau of the BOLD step response. In this way, we obtain a sufficient number of samples to train a classifier.

Figure 7.2 shows the plateau region from which samples are obtained. After the stimulus onset, there is the transient response, which reaches the plateau level after a certain time. Sampling takes place during the time interval indicated by the shaded region.

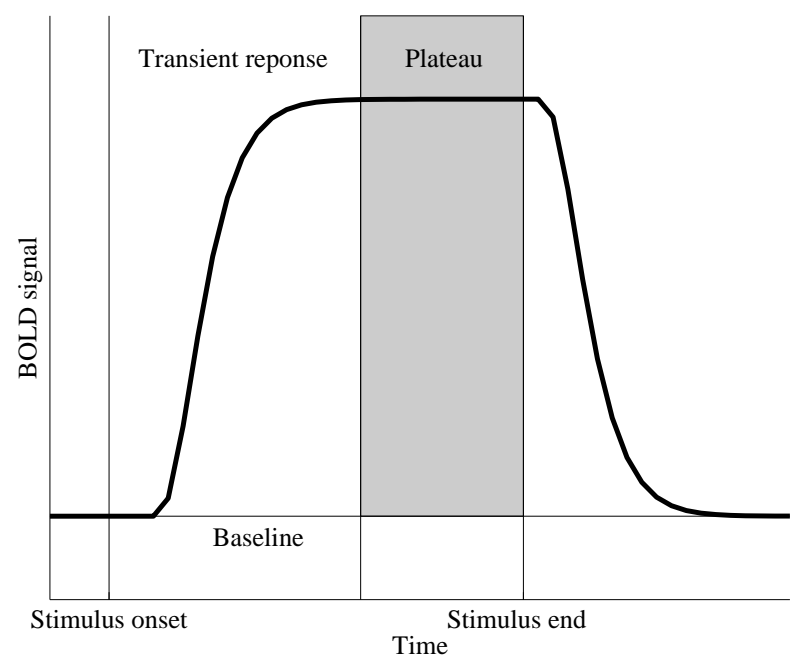

Figure 7.2: BOLD response in a block-design paradigm.

There are two design parameters involved in our sample selection. First, we need to select the amount of time between the stimulus onset and the start of the plateau phase; we will call this the TR offset. As the time constants in the BOLD response are not known a priori, we will use various values for the TR offset in our experiments.

Second, we have to define the number of samples to extract from the plateau. We expect that extracting more samples will allow us to train a classifier more reliably. However, given that the time constants in the BOLD response are not precisely known, we will use various values for this parameter as well.

\subsubsection{Feature selection}

The second modification involves the selection of features for classification. In contrast to the simulated-data experiments in the previous chapter, we cannot select features by setting our method's false-positive rate equal to that of the 
GLM, and then selecting features such that the detection rate is highest. The reason is that, in contrast to the simulated-data experiments, there is no spatial reference available in real fMRI data. We may solve this problem by making use of the experimental-protocol that was used in the creation of the dataset: we have knowledge on when each subject was subjected to what stimulus. This means that we know what the subject's brain state is, i.e., either "looking at face" (class 1, or $c_{1}$ ) or "looking at object" (class 2 , or $c_{2}$ ). We will select features based on the brain-state prediction quality of our method in the manner described below.

After training, our method is used to predict the brain states in a test set. The basic idea is to select features for classification such that the prediction of the brain state is best. We expect that features that predict the brain state well, are also good features to perform brain mapping. In detail, we use the method described in Subsection 6.4.3 to calculate the posterior probabilities $P_{i}\left(c_{1} \mid \bar{a}\right)$ and $P_{i}\left(c_{2} \mid \bar{a}\right)$ for each TR $i$ within a BOLD plateau in the test set. These probabilities from each of the TRs are then multiplied to obtain a $P\left(c_{1} \mid \bar{a}\right)$ and $P\left(c_{2} \mid \bar{a}\right)$ for the entire plateau, and thus for the entire stimulus exposure. The predicted brain state then is the class $c_{j}$ for which the posterior class probability is highest.

After the steps outlined above, we choose that particular feature fraction for which the class-prediction correctness is highest.

The selection procedure outlined above will be carried out using a form of cross validation. However, a note on this cross validation is in order. Theoretically, as we have 80 stimulus exposures, using wavelets for $n$ TRs each, we have $80 n$ samples. We could thus have performed a leave-one-out validation where we train on $80 n-1$ samples, and test on the remaining sample. However, as pointed out by Mitchell et al. (2004), this is unfair, since the correlation between the BOLD signal within a single exposure is so high that, effectively, one would be training on information that is in the test set. This same correlation problem exists within a single run, so training on $70 n$ samples and testing on $10 n$ samples would also give a result that is too optimistic. In preliminary experiments, we noticed that the correlation problem even extends to multiple runs of a single subject. Therefore, we decided to perform an inter-subject cross validation. That is, the brain-state classification folds are chosen such that when testing on the brain states of one subject, none of this subject's samples are in the training set.

\subsubsection{Scale determination}

The third modification pertains to the determination of the appropriate scale of specific regions. In principle, the scale-estimation method used in our experiments on real data, is the same as described in Subsection 6.4.4. However, in our dataset, a small face-specific region is positioned close to a large object-specific region. Gradient wavelets that cover both regions, will be specific (either very positively or very negatively). However, because the object-specific region is much larger than the face-specific region, the wavelets associated with the largest scale and region will be most specific. Applying the same scale-selection method as in Subsection 6.4.4 might thus lead to an over-estimation of the size of the smallest region. Preliminary experiments show that this over-estimation indeed occurs. 
We attempt to mitigate this problem by introducing an improved scale-estimation criterion that is expected to be less sensitive to adjacent different-sized areas. This criterion is that we regard gradient wavelets of scale $\sigma$ as being indicative of a region scale of $\sigma / 2$ (rather than $\sigma$, as in Subsection 6.4.4), because the positive or negative part of such a gradient wavelet is only half the size of the entire wavelet. We will return to this issue in Section 7.5.

\subsection{Experimental set-up}

In this section we present the set-up of our experiments. This set-up involves the choice of method-specific parameters presented in Subsection 7.3.1, and the definition of performance indicators presented in Subsection 7.3.2.

\subsubsection{Experimental procedure}

We face three choices in setting our method's parameters. First, we need to select the correct temporal section of the BOLD response (the "plateau" in Figure 7.2), which involves the selection of the $T R$ offset and the number samples afterwards (the TR number). We will use TR offsets of 1,4 , and 7 , and we will use TR numbers of $4,6,8,10,12$, and 14 .

Second, we have to select the type of features to use. Analogous to Subsection 6.5.1, we will perform experiments (1) with averaging wavelets only ("avg"), and (2) with both averaging and gradient wavelets ("alldir").

Third, because we use a multi-subject dataset, we have to choose whether to use cortical alignment, or not. Therefore, we will perform experiments both with, and without, using cortical alignment with a sphere-to-sphere mapping (SSM).

\subsubsection{Performance indicators}

In an experiment on real fMRI data, there is no spatial reference information available for specific regions. Therefore, we cannot quantitatively show our method's performance of voxel classification in terms of detection and false-positive rates. However, we can evaluate the performance of our region-based method using neuroscientific knowledge. This knowledge also aids us in assessing our scaleestimation technique in a qualitative manner.

Previous work has determined what brain areas are most likely involved in the discrimination between faces and objects. In Haxby et al. (1996), Halgren et al. (1999), and Haxby et al (2001), four regions were identified as being involved in the discrimination. Figure 7.3 shows the four regions involved. The fusiform face area (FFA) is specific to the processing of faces (Halgren et al., 1999), whereas the parahippocampal place area (PPA) is specific to the processing of objects (Haxby et al., 2001). Specifically in this left hemisphere, there are two additional face-specific areas that are related to the recognition of faces: an inferior-frontal region, and an anterior part of the visual cortex (Haxby et al., 1996). 


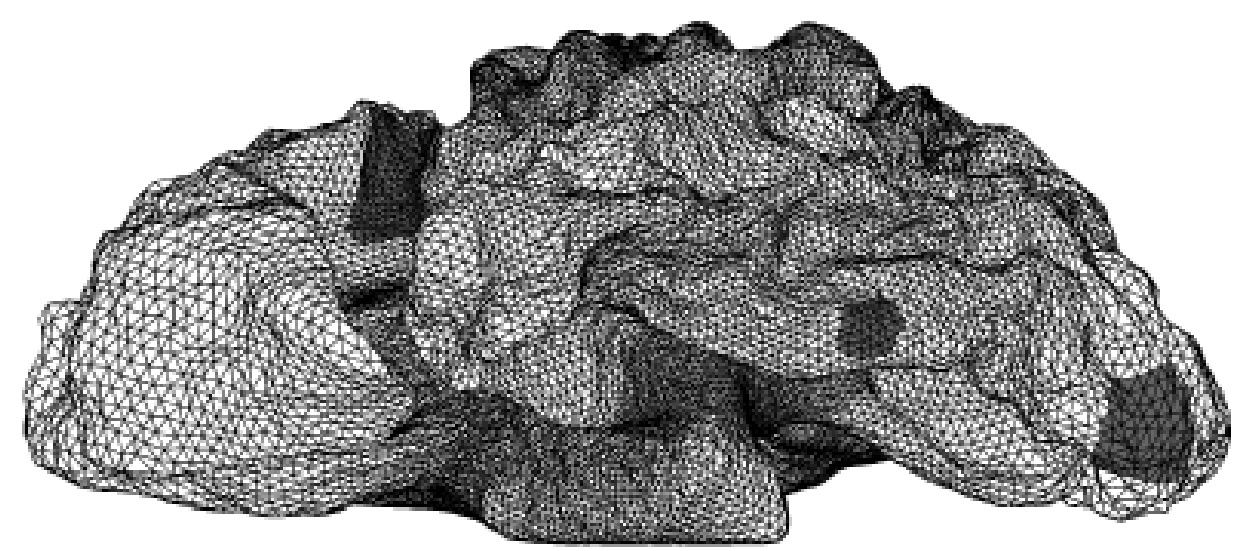

Figure 7.3: Brain regions established to be important in discriminating faces from objects. Dark grey: Face-specific regions. From left to right, the inferior-frontal region, the fusiform face area (FFA) and the anterior visual cortex. Light grey: Object-specific parahippocampal place area (PPA).

Again, we will compare the results of the region-based method with those obtained using the GLM. The performance criterion is whether each of the four regions is successfully identified as specific to the faces-objects task.

To evaluate the scale-estimation performance of our region-based method, we exploit the fact that the FFA is smaller than both the inferior frontal region and the anterior visual cortex, and that the PPA is larger than both the inferior frontal region and the anterior visual cortex. In a qualitative sense, this yields four performance indicators: (1) whether the FFA is estimated as smaller than the inferior frontal region, (2) whether the FFA is estimated as smaller than the anterior visual cortex (3) whether the PPA is estimated as larger than the inferior frontal cortex, and (4) whether the PPA is estimated as larger than the anterior visual cortex.

\subsection{Results}

In this section we present the results of our experiments. We will use the performance indicators introduced in Subsection 7.3.2 to assess the region-based method's performance in both specific-region detection and region-scale estimation. In Subsection 7.4.1, we present the results on specific-region detection. In Subsection 7.4.2, we present the results on region-scale estimation. 


\subsubsection{Specific-region detection results}

In this subsection, we compare the specific-region detection performance of our method to that of the GLM. Table 7.1 shows the specific-region detection results. The results of the experiments are represented in terms of circles. Each circle represents the results of a specific experimental setting. In an anti-clockwise fashion, the four quadrants of a circle represent the following states.

- The FFA is visible.

- The anterior visual cortex is visible.

- The inferior frontal region is visible.

- The PPA is visible.

Unshaded (white) quadrants represent a successful detection of the associated area. The more quadrants are unshaded, the better the overall detection result is.

Figure 7.4 shows the brain map obtained using the GLM, which represents the baseline to which our results are compared. The top panel shows the face-specific regions detected by the GLM in white, and the bottom panel shows the objectspecific regions detected by the GLM in white. It is clear that while the GLM does detect the large inferior frontal region, the anterior visual cortex, and the PPA, it does not detect the small FFA. In addition, there are some false positives superior to the anterior visual cortex.

Table 7.1 clearly shows our main result. For the appropriate choice of parameters, our method outperforms the GLM in region-detection performance. The region-based method is capable of detecting the small FFA, whereas the GLM is not. In addition to this main result, we make four observations from the results in the table.

First, using both averaging and gradient wavelets yields maps on which the four important brain regions are identified better than when using averaging wavelets only. In passing, we remark that this result is consistent with the results obtained on simulated data in the previous chapter. We conjecture that there are two possible reasons for this difference in performance. First, the use of gradient wavelets allows one to detect the edges of specific regions. Second, as introduced in Subsection 7.2.3, our dataset contains the activations of two adjacent areas that respond specifically to different stimuli. Gradient wavelets, unlike averaging wavelets, allow for the detection of the difference of specificity of these two regions.

Second, the use of an SSM correction does improve the identification results, although results without SSM correction are only slightly worse than results with SSM correction. The fact that SSM correction improves the detection results is not surprising, since SSM correction reduces the spatial variance among the brain regions of the different subjects. The fact that results are still rather good without SSM correction is a consequence of the fact that our method is region based; our features cover larger spatial areas than those in a vertex-based method, and are thus more robust against small displacements of the underlying cortex. This result is important, since it shows that our method, by its nature, is more robust 


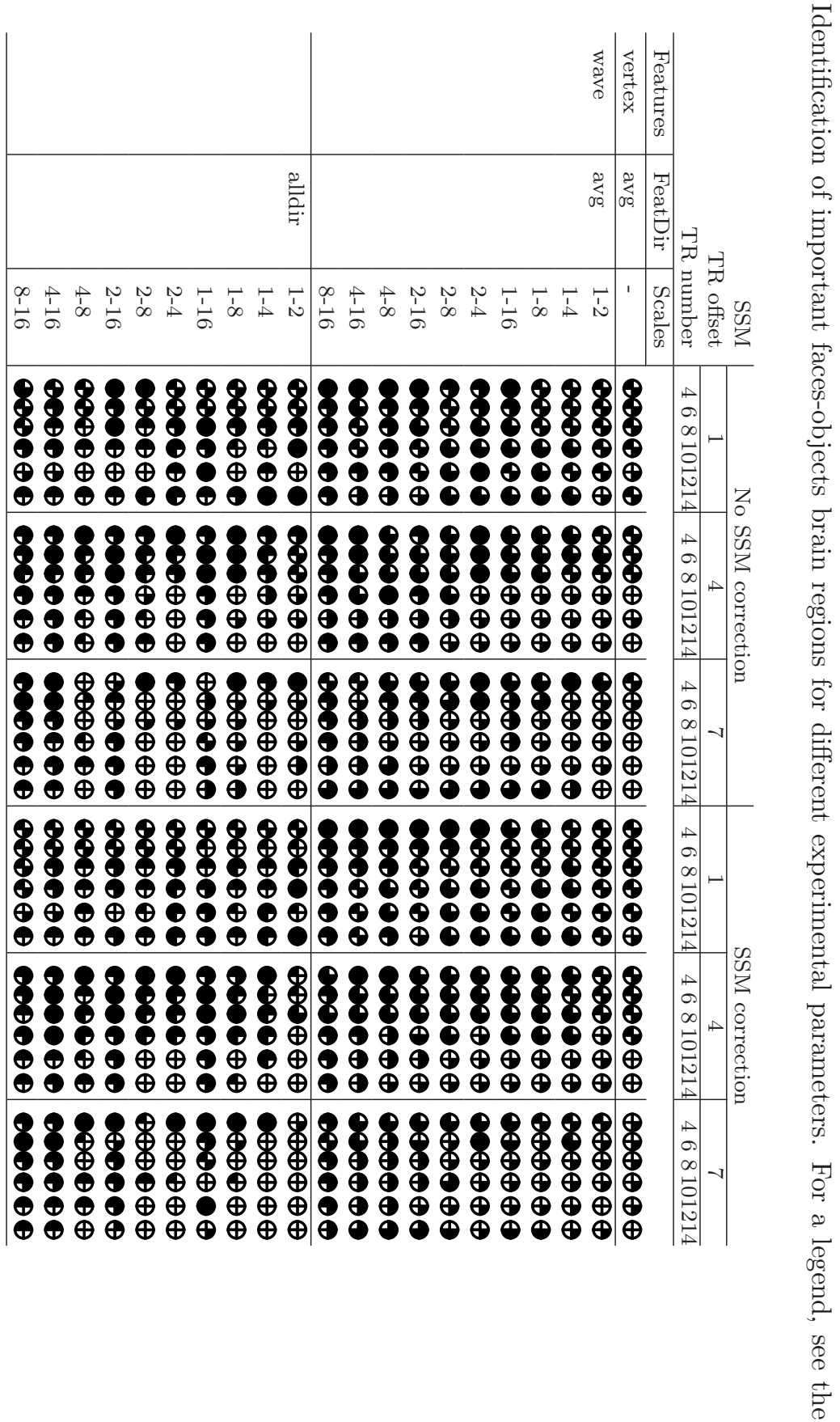




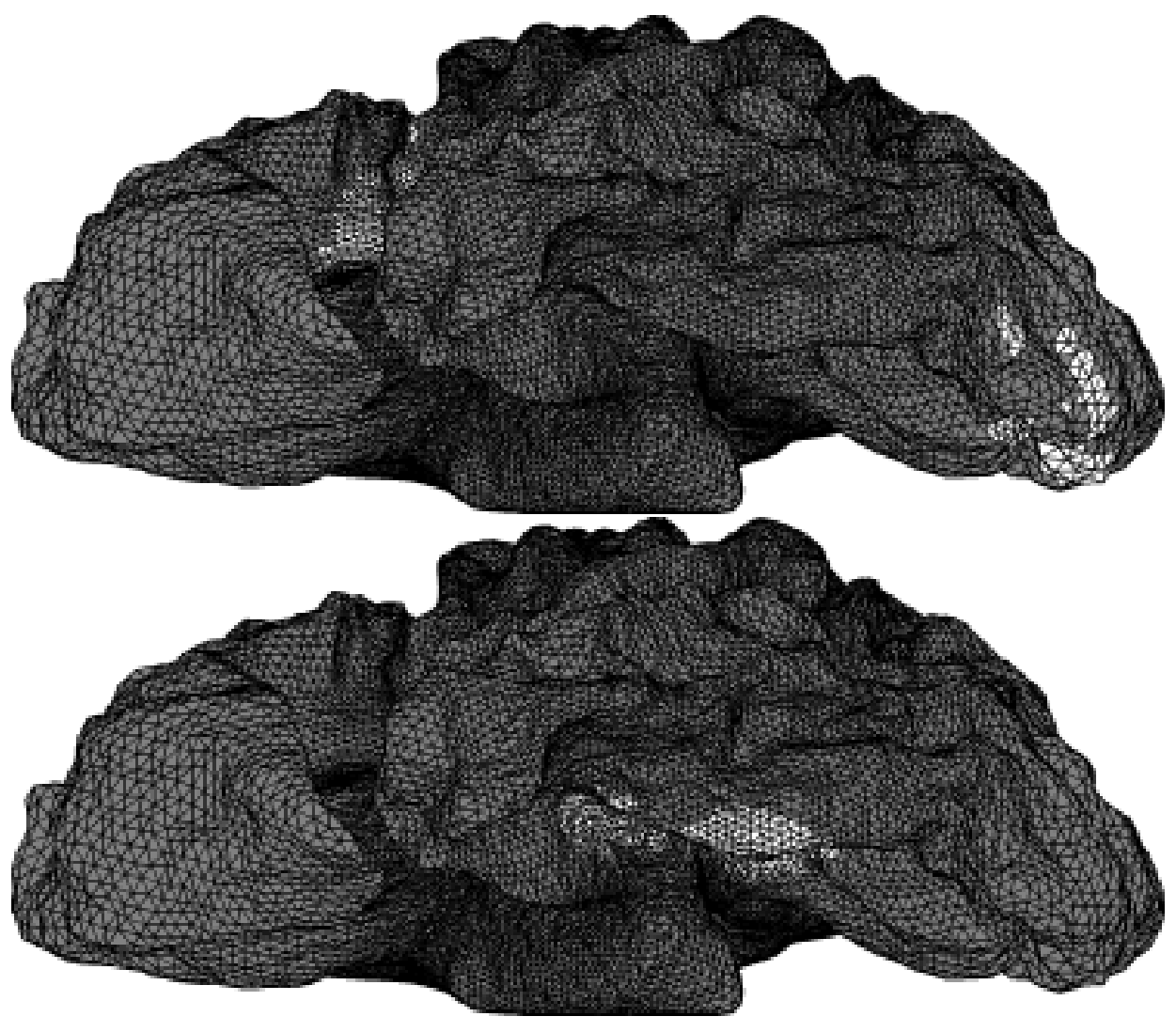

Figure 7.4: Brain specificity map obtained with the GLM. Top: face-specific areas. Bottom: object-specific areas. 
to inter-subject anatomical differences than traditional vertex-based methods. We will elaborate on this point in Section 7.5.

Third, the results show that choosing the correct section of the BOLD plateau for feature extraction is an important determinant for the region-identification quality. The first parameter influencing the BOLD-plateau section is the number of TRs. The more TRs we use to extract features from, the more samples we obtain, and the more reliably our posterior-probability estimates become. This is reflected in the results: detection results are better as more TRs are used for practically all parameter settings. The second parameter influencing the BOLDplateau selection is the TR offset. That is, the number of TRs that are discarded after the onset of the stimulus. It is clear from the results that region-detection quality is best when an offset of $7 \mathrm{TRs}$ is used. This is readily explained as follows. As there is considerable difference among the BOLD responses in the first transient (step-response) phase, and less so in the level (static) phase, excluding the transient phase yields a higher effective SNAR and inter-subject variability. This improves the detection results. Apparently, discarding the first 7 TRs is necessary to exclude all transient BOLD effects.

Fourth, the optimal wavelet scales for discriminating-region detection seem to be rather small. In descending order of region-detection quality, these scales are $1-2,1-4,1-8$ and $2-4,1-16$, and $2-16$. In addition, the optimal scales for SSM-corrected experiments are smaller than those for non-corrected experiments. This is in line with our expectations, since the spatial variability among subjects is higher without SSM correction, so larger wavelets are most specific to correct for this variability. In contrast, for SSM corrected data, the variability is less, so smaller wavelets can be reliably used.

Figure 7.5 shows a brain map obtained by using our method. The top image shows the areas identified as face specific in white. The bottom image shows the areas identified as object specific in white. When comparing this map to the multi-subject GLM map in Figure 7.4, it is clear that whereas the GLM detects only three of the four specific brain regions, the region-based method detects all four specific brain regions.

\subsubsection{Scale-estimation results}

The scale-estimation results are displayed in Table 7.2. The estimated scales are displayed in a manner similar to that used in Table 7.1. The quadrants of each circle now represent the quality of relative scale estimation according to the performance indicators introduced in Subsection 7.3.2. Quadrant 1 is unshaded if the scale of the anterior visual cortex region is (correctly) estimated as larger than the scale of the FFA. Quadrant 2 is unshaded if the scale of the inferior frontal region is (correctly) estimated as larger than the scale of the FFA. Quadrant 3 is unshaded if the scale of the inferior AT region is (correctly) estimated as larger than the scale of the anterior visual cortex. Finally, quadrant 4 is unshaded if the scale of the inferior AT region is (correctly) estimated as larger than the scale of the inferior frontal region. The more quadrants are white, the better the scale-estimation performance of our method is. 


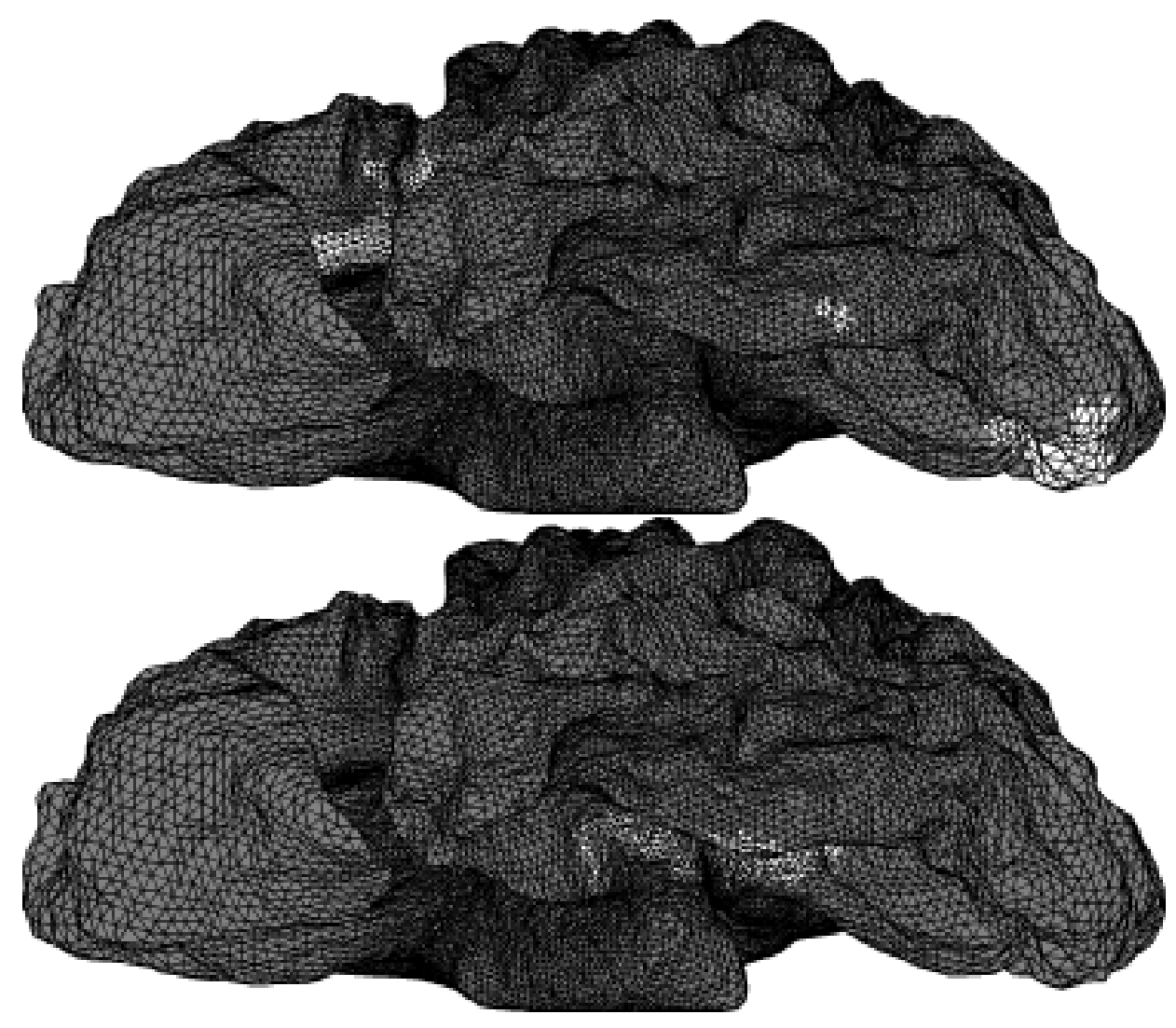

Figure 7.5: Brain specificity map obtained with the region-based method. Top: facespecific areas. Bottom: object-specific areas. 


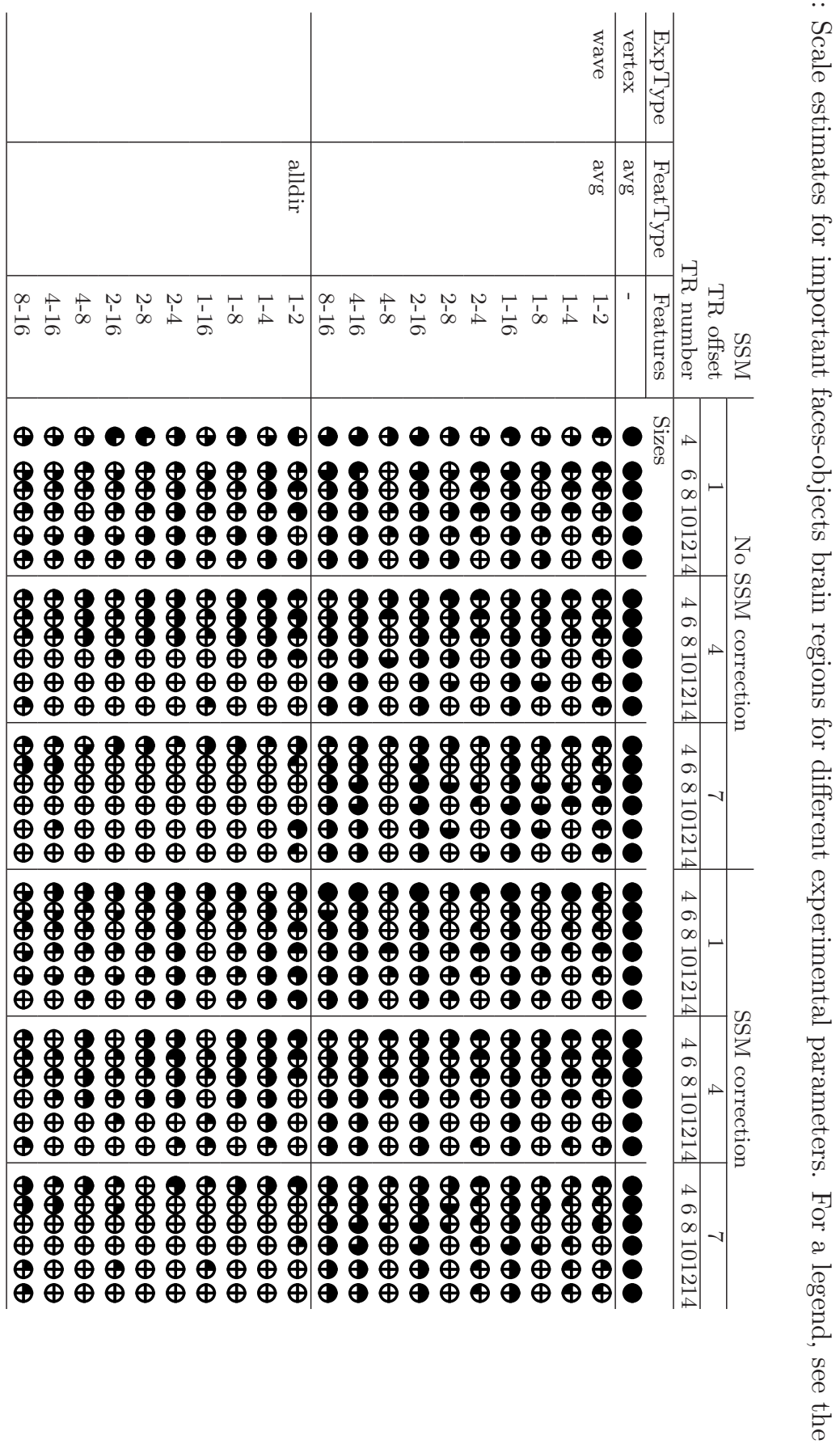


The main result is that, under suitable parameter conditions, our method is capable of correctly identifying the scale differences among the four specific regions. Furthermore, this result is obtained without having to select a statistical threshold on the specificity. As explained in Section 6.1, one has to set a threshold on the specificity in all current methods that influences the estimated region size. In contrast, our method obtains a scale estimate independent of such a threshold. In addition to this main result, we make four additional observations.

First, the inclusion of both averaging and gradient wavelets improves the relative scale-estimation quality compared to the use of averaging wavelets only (this is as in the region-identification results shown above). We expect that this is due to the same two reasons: (1) the fact that the edges of specific regions are described better by gradient wavelets, and (2) the fact that the FFA and PPA are adjacent regions.

Second, SSM correction does slightly improve the relative scale-estimation results, although the results without SSM correction are good as well. (this is as in the region-identification results shown above). The fact that scale-estimation results are rather good even without using an SSM correction is an important result. It means that our method is capable of reliably estimating region scales despite inter-subject anatomical differences; a property in which our method is currently unique.

Third, the relative scale-estimation results show a similar sensitivity to the selection of the appropriate BOLD region as the region-identification results shown in Subsection 7.4.1. In general, the best results are obtained using a TR offset of 7 , and a many TRs as possible.

The fourth observation is strikingly different from that made in the regionidentification results shown above, and concerns the optimal wavelet scales. Good results are obtained for almost all scales, except when very large scales (notably 16) are included. We assume that the largest wavelets are larger than any functional unit in the brain, and thus do not convey useful information, but rather incorporate noisy data from unspecific regions.

\subsection{Discussion}

In this section, we discuss the results obtained in the previous section. In particular, we discuss three issues of relevance to the results obtained. First, we discuss the ability of the region-based method to deal with inter-subject variations (Subsection 7.5.1). Second, we evaluate the adapted scale-estimation criterion employed (Subsection 7.5.2). Finally, we discuss how the choice for left-hemisphere data may have affected our results (Subsection 7.5.3).

\subsubsection{Inter-subject variations}

One of the research questions in this chapter is whether our method is capable of dealing with inter-subject differences in a better way than traditional methods. In order to address the issue of inter-subject variability properly, it is important to note that these differences can be functional and anatomical (Hunton et al., 1996). 
Functional differences exist where different subjects process the same stimulus in functionally different parts of the brain. These differences occur mostly in higher brain functions, and mostly in the anterior part of the brain. In our experiment, we do not face the problem of having to deal with functional differences; our method deals with brain functions that reside in well-defined areas of the brain.

Anatomical differences are small differences in the shape of the brain, e.g., slightly different shapes of sulci and gyri, and differences in the overall proportions of the brain. The cortical mapping, as described in Subsection 7.1.3, is capable of reducing these differences to a large degree. Nevertheless, even after alignment, functional areas will not map exactly to one another across subjects. This may make the detection of, in particular, small specific areas problematic.

In the previous section, we established that our method does detect the small face-specific FFA, whereas the GLM fails to do so. Moreover, our method detects the FFA even without cortical realignment. Preliminary experiments have shown that the difference between aligned and non-aligned data is in the order of one to a few grid units, which corresponds to the radius of the FFA. Therefore, a vertexbased method such as the GLM, may not be able to establish even one vertex that is specific across all subjects. In contrast, in our method a wavelet somewhat larger than the FFA, centred on the average FFA position across subjects, will still have a significant specificity. Therefore, our method's region-based nature enables the detection of small specific areas despite cortical differences. Even after realignment, there are inter-subject differences, to which our method is more robust.

In order to investigate our claim that the reason for the GLM's failure to detect the FFA, is due to inter-subject anatomical differences, we performed the following experiment. We applied the GLM to the four runs of one of our subjects, thus ruling out inter-subject differences. The brain map is shown in Figure 7.6.

From the figure, it is clear that the GLM now does correctly identify the FFA. We consider the outcome of this small experiment as sufficient evidence to believe that the GLM's failure to detect the FFA in a multi-subject experiment, is indeed due to the inter-subject anatomical differences.

\subsubsection{Scale calculation}

As mentioned in Subsection 7.2.3, our experiments on real data are performed using a slightly different scale-estimation criterion. In the previous chapter, a wavelet of scale $\sigma$ was regarded as indicative of a region scale of $\sigma$, regardless of the wavelet type. In this chapter, averaging wavelets of scale $\sigma$ are still regarded as indicative of a region scale of $\sigma$, but gradient wavelets are regarded as indicative of a region scale of $\sigma / 2$. In Subsection 7.2 .3 we suggested that adjacent taskspecific regions of different sizes (i.e., the large PPA is adjacent to the small FFA) may pose a problem for scale estimation. Large gradient wavelets that cover both regions, may lead to an overestimation of the scale of the smaller region.

In order to test this suggestion we created a brain map based on gradient wavelets only. More specifically, we used wavelet scales $2-8$, SSM correction, a TR offset of 7 , and 14 TRs per exposure. Moreover, we selected the top 1/1,000 


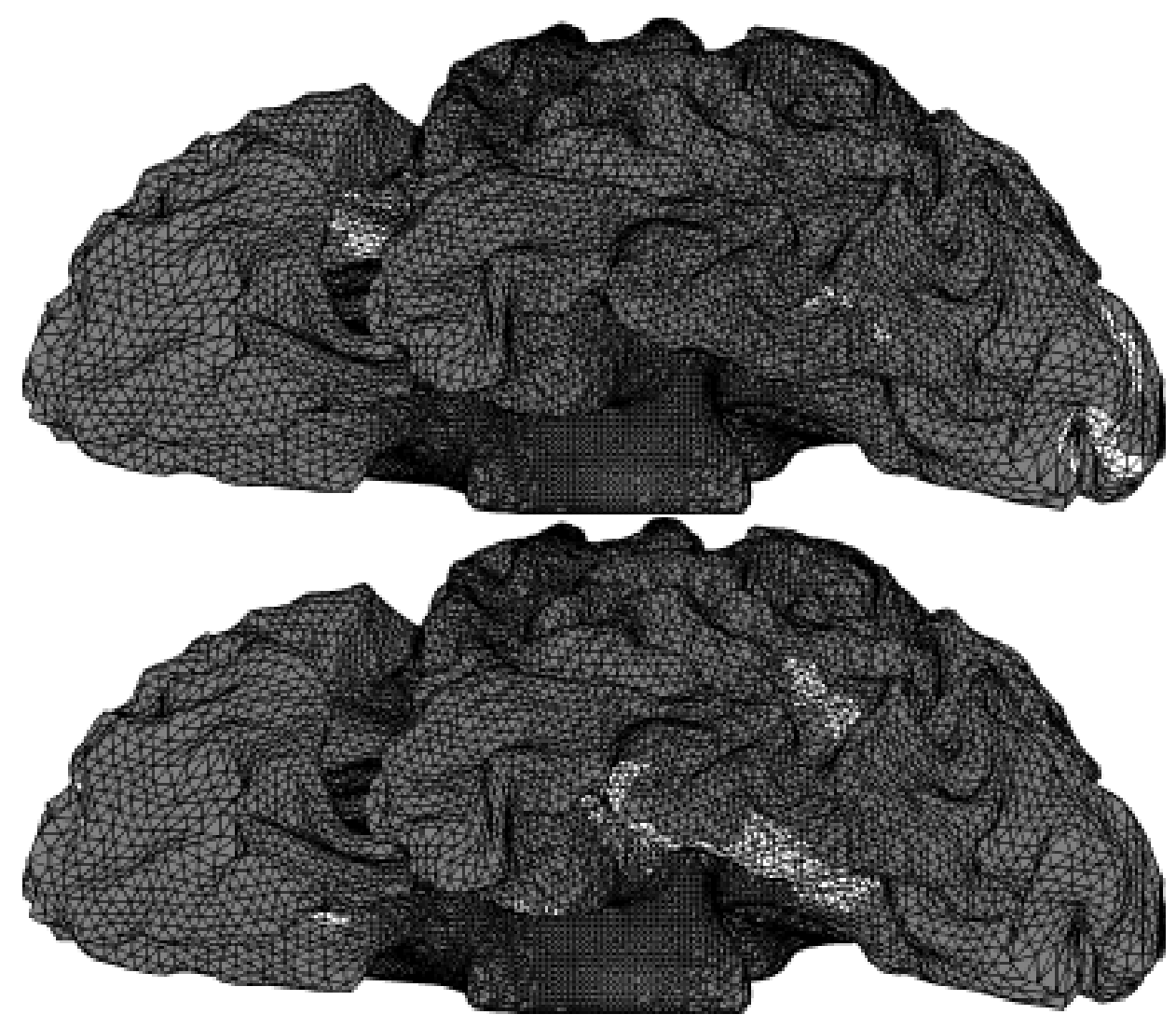

Figure 7.6: Brain specificity map obtained with the GLM on four runs of a single subject. Top: face-specific areas. Bottom: object-specific areas. 
fraction of most specific features, and from those, created a brain map based on gradient wavelets only. The result is shown in Figure 7.7.

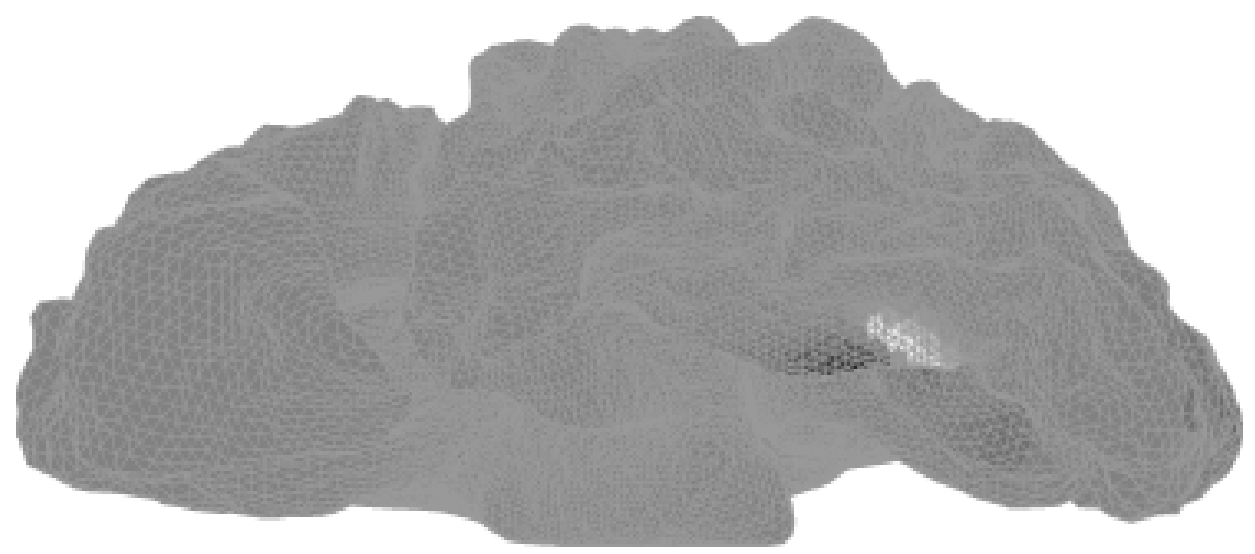

Figure 7.7: Brain specificity map obtained using the region-based method, showing the effect of gradient wavelets only.

In the figure, grey represents non-specific areas, white represents face-specific areas, and black represents object-specific areas. The figure supports the claim that the proximity of the FFA and PPA causes many wavelets overlapping these two areas to be specific. Moreover, $79 \%$ of these wavelets are of scale 8 , even though the FFA is of scale 3 to 4 at the most. Therefore, it is clear why regarding gradient wavelets of scale $\sigma$ as indicative of a region scale of $\sigma / 2$, works better in our experiment on real data.

Scale estimation in our method should be improved to estimate the characteristic scale in an anisotropic manner, rather than estimating one isotropic scale estimate for the region. This can be done by estimating different scales of each of the three grid directions. Such a procedure could allow for a more reliable classification of highly elongated specific regions. Future research should focus on automatically estimating the characteristic scale in an anisotropic manner.

\subsubsection{Choice of brain hemisphere}

The experiments in this chapter have all been performed on data from left hemispheres, because the left hemisphere shows more specific activations for the task at hand than the right hemisphere. In particular, the inferior temporal-region specificity is unilateral to the left hemisphere (Haxby et al., 1996). In addition, the FFA response is often less pronounced in the left hemisphere (Haxby et al., 1996). Because of these two reasons, data from the left hemisphere thus provides a more challenging task for a brain-mapping algorithm, showing performance differences between methods more clearly.

The fact that the region-based method outperforms the GLM under difficult conditions implies that our method might be usable under even more difficult 
conditions. In particular, we recommend to apply our method to datasets in which the GLM does not produce useable results.

\subsection{Chapter conclusions}

In this chapter, we applied our region-based brain mapping method to real fMRI data, obtained from a multi-subject faces-objects discrimination experiment.

The results indicate that our method outperforms the traditional GLM in terms of the quality of detection of task-specific regions. In addition, our method yields useful scale estimates for each of the specific regions, without having to define statistical thresholds on the specificity. Therefore, the answer to research question RQ5 is that our method generalises well to actual data, both in terms of specific-region detection, and in terms of scale estimation.

With regard to research question RQ6, our method is significantly more robust to inter-subject anatomical differences than the standard vertex-based GLM. In particular, our method is capable of finding small specific brain areas, such as the fusiform face area, despite anatomical differences whose lateral dimensions are in the same order to magnitude as the size of the specific region. The robustness of our method with regard to inter-subject anatomical differences is made more apparent by the fact that it performs well even without using specialised cortical alignment.

The robustness to inter-subject anatomical differences is due to two reasons. First, our method is multivariable, and uses features that are both spatially extended, and take spatial-relation information into account. This allows for the proper identification of regions despite significant differences in the regions' location among subjects. Second, our method employs an efficient heuristic on the Bayesian algorithm, that effectively deals with noisy data.

To sum up, we may draw the following seven conclusions. (1) For the appropriate choice of parameters, our method outperforms the GLM in region-detection performance. The region-based method is capable of detecting the small FFA, whereas the GLM is not. (2) Under suitable parameter conditions, our method is capable of correctly identifying the scale differences among the four specific regions, whereas vertex-based methods such as the GLM are not. (3) Although the application of cortical alignment via SSM corrections does improve both the specific-region detection and the region-scale estimation, our method is unique in that it yields good specific-region detection and region-scale estimation even without cortical alignment. (4) The use of averaging and gradient wavelets together, rather than just averaging wavelets, yields (a) maps on which the four important brain regions are identified better, and (b) better relative-scale estimates for taskspecific brain regions. (5) Choosing the correct section of the BOLD plateau for feature extraction is an important determinant for the region-identification quality. The use of as many samples as possible from the BOLD plateau improves both the specific-region detection quality, and the scale-estimation quality. (6) The optimal wavelet scales for specific-region detection is rather small (1 to 2 grid units). (7) The choice wavelet scales for region-scale estimation is less critical 
than for specific-region detection. Good results are obtained for almost all scales, except when very large scales (notably 16) are included. 


\section{Chapter 8}

\section{General discussion of the region-based method}

In this chapter we discuss of the region-based method in general. In Section 8.1 we discuss the relation between noise and our manner of sampling. In Section 8.2 we discuss issues related to our spherical wavelets. In Section 8.3 we discuss our classifier structure based on Bayesian statistics, and its relation to massively univariate and multivariate methods. In Section 8.4 we put the region-based method into perspective, by discussing its relevance in relation with similar work. Finally, in Section 8.5 we explore subjects for future work.

\subsection{Sampling and noise issues}

In this section we elaborate on two noise-related issues. In Subsection 8.1.1 we discuss how our sampling method interacts with the background noise present in fMRI signals. In Subsection 8.1.2, we present a possible mechanism to correct for background noise.

\subsubsection{Sampling and noise}

There are two sampling issues involved when we convert our data from a volume time course to a mesh time course. First, there is the problem that the automatically determined location of the cortical surface will never be precise. In locations where there is a mismatch between the estimated and actual cortical surface, small volume activations around the actual cortex may not be sampled sufficiently on the estimated cortex location. This may present problems in finding small areas, such as the Fusiform Face Area. In the Chapters 6 and 7, we mitigated this problem by applying a small amount $(2.8 \mathrm{~mm})$ of volumetric smoothing before sampling the VTC to the MTC. Although volumetric smoothing at too large a scale may present small specific regions to be found, our results indicate that small regions are found reliably at the current smoothing scale. 
The second problem is that of noise correlations. A time course for a vertex in the MTC always consists of a linear combination of time courses of surrounding VTC voxels. This implies that the temporal noise in VTC voxels will become spatially correlated in the MTC. When pre-smoothing the VTC, in the manner we do, this effect is enlarged. Clearly, when using overlapping wavelets, noise will be correlated among wavelet coefficients as well. However, our results indicate that our region-based approach improves detection results, despite the increased noise correlation.

\subsubsection{Weighting for noise}

The magnitude of temporal background noise in the BOLD signal varies across the brain. In its current form, the region-based method does not take the noise magnitude into account. While the results obtained in the experiments on simulated and real fMRI data are comparable to the GLM in terms of region-detection quality, the results might be further improved by taking the noise into account. In this section, we establish whether this is the case. The basis idea is to regard features that overlap a brain region with a high background-noise magnitude, as less descriptive. Taking noise into account is performed in two steps. First, a weighting coefficient is computed. Second, this weighting coefficient is applied in the learning algorithm. We describe both steps below.

A noise-weighting coefficient $w$ is calculated for each feature. This is done by first calculating the noise magnitude per vertex, and then using these values to calculate $w$. The noise magnitude per vertex is calculated as the standard deviation of the BOLD signal for that vertex, during resting-state periods. The coefficient $w$ for each feature is the average of the noise magnitude of all vertices that the wavelet covers. The calculation of these averages can be performed in an efficient manner, by applying the integral-image framework to the measured vertex standard deviations.

The noise-weighting coefficients $w$ that were obtained for each feature are subsequently applied as follows. First, the normal naive-Bayesian training as described in Subsection 6.4.2 is performed. The log-likelihood values $\ell$ obtained after Bayesian training are then weighted by the noise-weighting coefficient; they are divided by $(1+0.1 w)$. Subsequently, feature grouping and classification of specific regions takes place as before.

We have performed a preliminary study to assess the effect of noise weighting on simulated fMRI data. The preliminary results are shown in Table 8.1. The results can be compared to those in Table 6.3 , where noise weighting was not performed. There are two points to note in the results. First, noise weighting seems to improve the results on the brain with unequally sized regions only. The reason for this is unknown. Second, for high CNARs, the noise-weighted method yields worse detection performance than the unweighted method. The reason for this is unclear. Third, between CNARs of 0.2 and 0.5 , noise weighting improved the detection rate considerably over all wavelet scales.

Table 8.2 shows the region-scale estimates obtained using the noise-weighted method. The results can be compared to those in Table 6.4, where noise weighting 
Table 8.1: Detection-rate results for each of the two patterns of activation, for several wavelet scales, and for all combinations of SNARs and CNARs - weighted for noise.

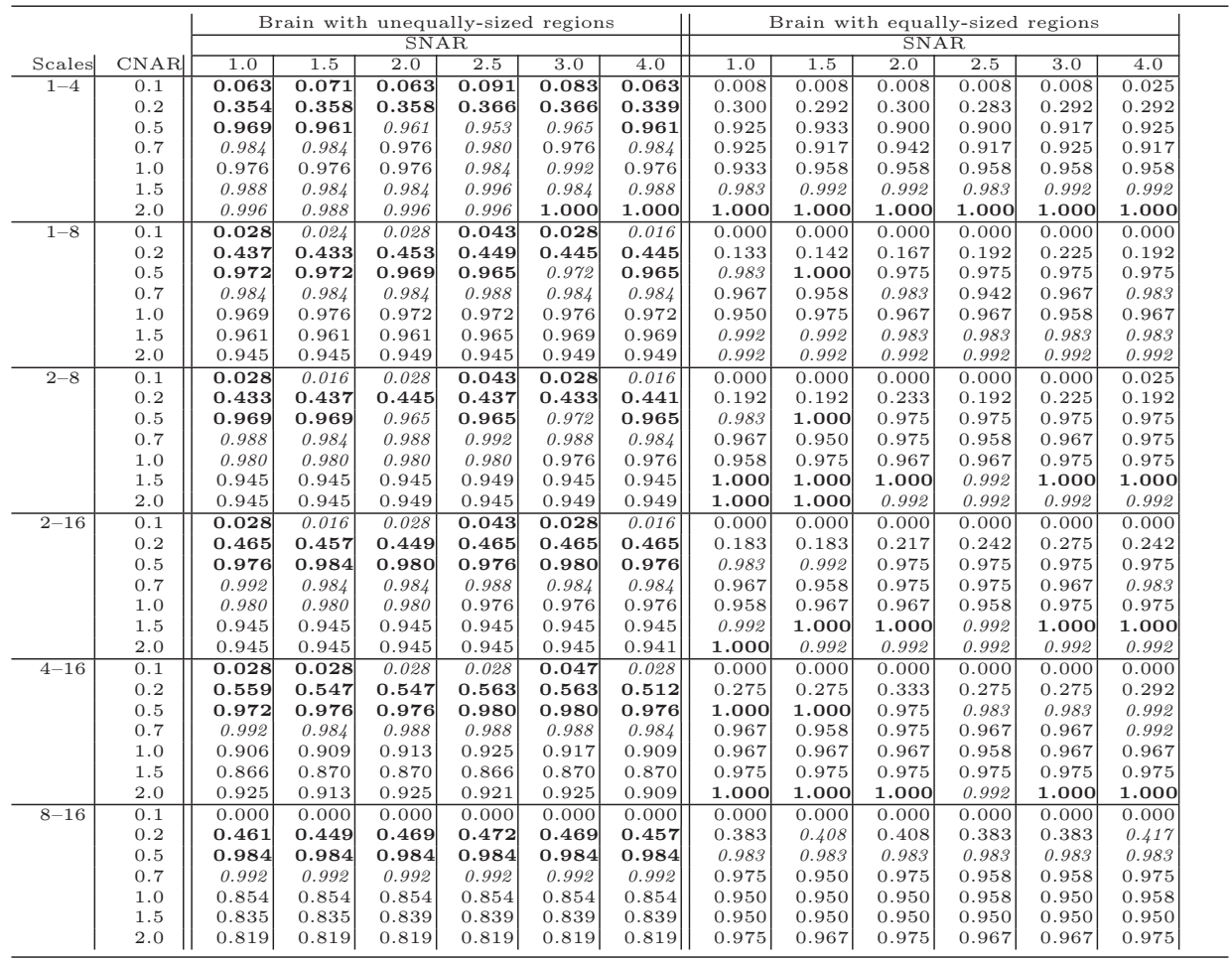

was not performed. Noise weighting improves the scale estimates considerably for the brain with equally sized regions. For the brain with unequally sized regions, scale estimation improves for wavelet scales $2-16$ and $4-16$. For wavelet scales $2-16$, the scale estimates are best for both patterns of activation.

From these preliminary results, we may conclude that taking the magnitude of background noise into account can be instrumental in improving the results. We therefore recommend this method for implementation in a future version of the region-based method.

\subsection{Wavelet-feature issues}

In this section we discuss two issues related to the wavelet features we employ. In Subsection 8.2.1, we discuss the differences between (1) using averaging wavelets only, and (2) using both averaging and gradient wavelets. In Subsection 8.2.2 we discuss two theoretical points of our choice of wavelets in relation to the classical wavelet theory. In Subsection 8.2.3, we discuss the spatial-smoothing properties of the wavelets. 
Table 8.2: Region-scale estimation results for each of the two patterns of activation, for several wavelet scales, and for all combinations of SNARs and CNARs - Weighted for noise.

\begin{tabular}{|c|c|c|c|c|c|c|}
\hline $\begin{array}{l}\infty \\
\vdots \\
\vdots\end{array}$ & $\begin{array}{l}\Delta \\
\Delta \\
\end{array}$ & $\begin{array}{c}\tilde{N} \\
\omega \\
\omega\end{array}$ & 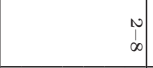 & $\overrightarrow{+}$ & 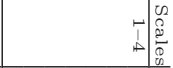 & \\
\hline 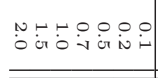 & 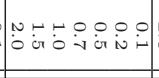 & 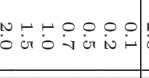 & 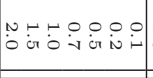 & 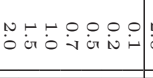 & 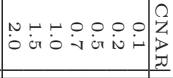 & \\
\hline 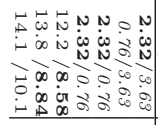 & 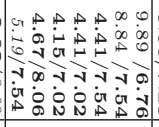 & 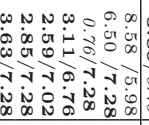 & 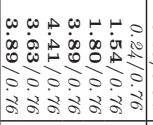 & 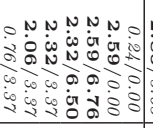 & 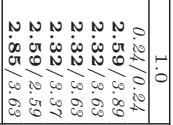 & \\
\hline 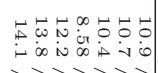 & 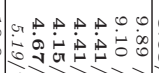 & 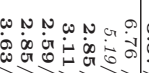 & 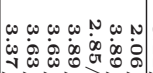 & 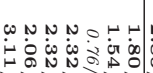 & 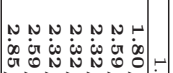 & \\
\hline 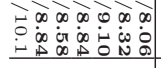 & 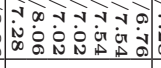 & 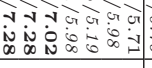 & 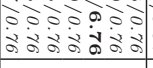 & 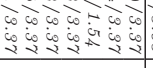 & 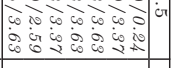 & . \\
\hline 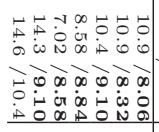 & 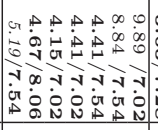 & 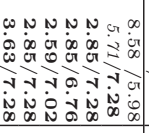 & 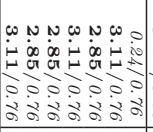 & 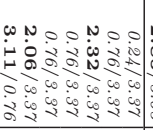 & 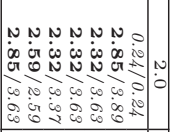 & - \\
\hline 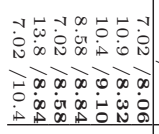 & 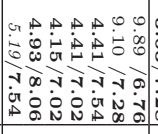 & 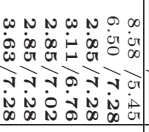 & 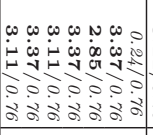 & 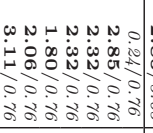 & 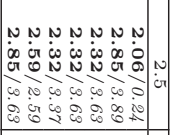 & of \\
\hline 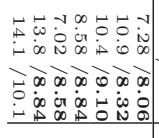 & 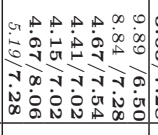 & 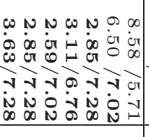 & 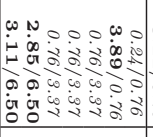 & 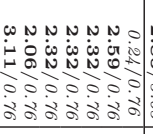 & 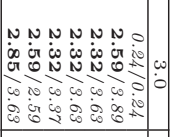 & 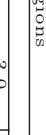 \\
\hline 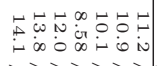 & 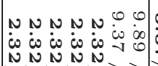 & 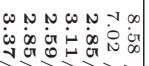 & 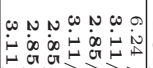 & 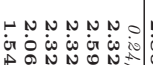 & 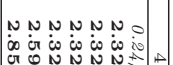 & \\
\hline 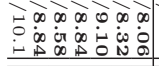 & 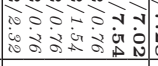 & 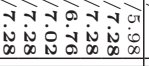 & 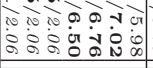 & & 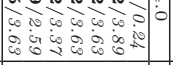 & \\
\hline 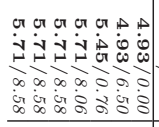 & 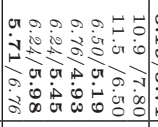 & 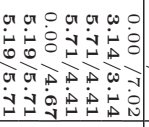 & 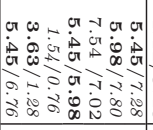 & 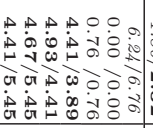 & 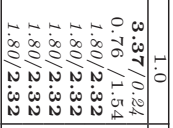 & \\
\hline 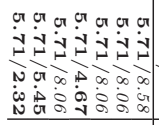 & 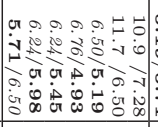 & 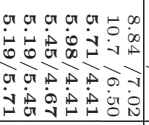 & 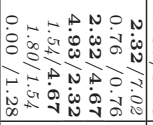 & 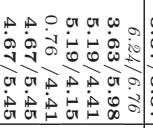 & 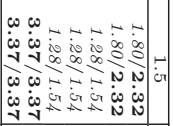 & \\
\hline 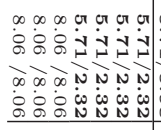 & 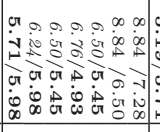 & 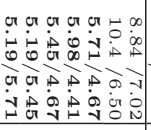 & 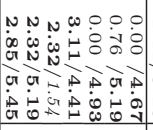 & 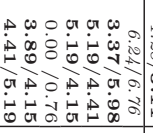 & 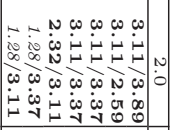 & - \\
\hline 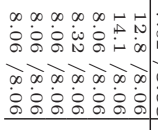 & 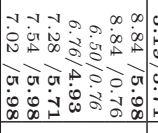 & 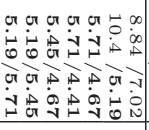 & 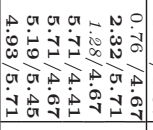 & 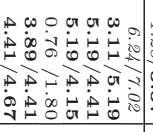 & 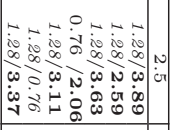 & \\
\hline 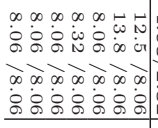 & 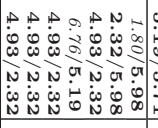 & 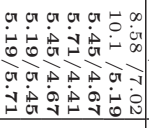 & 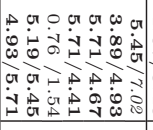 & 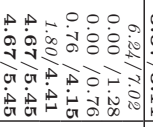 & 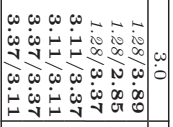 & \\
\hline 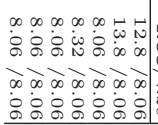 & 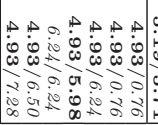 & 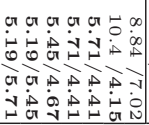 & 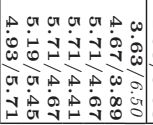 & 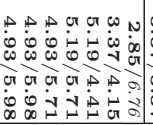 & 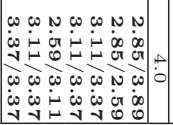 & \\
\hline
\end{tabular}




\subsubsection{Wavelet types}

In this section we investigate whether the use of both averaging and gradient wavelets - as in the experiments above - is pivotal for our method to function well, or whether using averaging wavelets alone suffices. To this end, we compare results obtained from experiments using averaging wavelets alone to results obtained from experiments using both types of wavelets.

In our experiments on simulated data in Chapter 6, we used both averaging and gradient wavelets. We subsequently obtained results using averaging wavelets only. Tables 8.3 and 8.4 show representative results for the specific-region detection rate, and the region scale estimation, respectively. The tables show results for the brain with unequally-sized regions, for wavelet scales on the dyadic grid from 2 to 16 .

Table 8.3: Detection-rate results for the brain with unequally-sized regions, for all combinations of SNARs and CNARs, when only averaging wavelets are used.

\begin{tabular}{|c|c|r|r|r|r|r|r|}
\multicolumn{2}{|c|}{ Scales } & \multicolumn{7}{|c|}{ CNAR } & \multicolumn{1}{|c|}{1.0} & \multicolumn{1}{c|}{1.5} & \multicolumn{1}{c|}{2.0} & \multicolumn{1}{c|}{2.5} & \multicolumn{1}{c|}{3.0} & \multicolumn{1}{c|}{4.0} \\
\hline $2-16$ & 0.1 & 0.000 & 0.000 & 0.000 & 0.000 & 0.000 & 0.000 \\
& 0.2 & $\mathbf{0 . 2 3 2}$ & $\mathbf{0 . 2 1 7}$ & $\mathbf{0 . 2 1 3}$ & $\mathbf{0 . 2 1 7}$ & $\mathbf{0 . 2 1 7}$ & $\mathbf{0 . 2 2 4}$ \\
& 0.5 & $\mathbf{0 . 9 7 2}$ & $\mathbf{0 . 9 6 9}$ & $\mathbf{0 . 9 6 9}$ & $\mathbf{0 . 9 6 9}$ & 0.969 & $\mathbf{0 . 9 7 2}$ \\
& 0.7 & 0.996 & 0.996 & 0.996 & 0.996 & 0.996 & $\mathbf{1 . 0 0 0}$ \\
& 1.0 & 0.992 & 0.992 & 0.992 & 0.992 & 0.996 & 0.992 \\
& 1.5 & $\mathbf{1 . 0 0 0}$ & $\mathbf{1 . 0 0 0}$ & $\mathbf{1 . 0 0 0}$ & $\mathbf{1 . 0 0 0}$ & $\mathbf{1 . 0 0 0}$ & $\mathbf{1 . 0 0 0}$ \\
& 2.0 & $\mathbf{1 . 0 0 0}$ & $\mathbf{1 . 0 0 0}$ & $\mathbf{1 . 0 0 0}$ & $\mathbf{1 . 0 0 0}$ & $\mathbf{1 . 0 0 0}$ & $\mathbf{1 . 0 0 0}$ \\
\hline
\end{tabular}

Table 8.4: Scale-estimation results for the brain with unequally-sized regions, for all combinations of SNARs and CNARs, when only averaging wavelets are used.

\begin{tabular}{|c|c|c|c|c|c|c|c|}
\hline \multirow{2}{*}{ Scales } & CNAR & \multicolumn{7}{|c|}{ SNAR } \\
\cline { 2 - 8 } $2-16$ & 0.1 & $8.58 / \mathbf{7 . 8 0}$ & $8.58 / \mathbf{7 . 8 0}$ & $8.58 / 7.80$ & $8.58 / 7.80$ & $8.58 / 7.80$ & $8.58 / \mathbf{7 . 8 0}$ \\
& 0.2 & $8.06 / 5.71$ & $8.06 / 5.71$ & $8.32 / 5.71$ & $8.06 / 5.71$ & $8.06 / 5.71$ & $8.32 / 5.71$ \\
& 0.5 & $6.50 / 5.98$ & $6.50 / 5.98$ & $6.50 / 5.98$ & $6.50 / 5.98$ & $6.24 / \mathbf{6 . 2 4}$ & $6.50 / 5.98$ \\
& 0.7 & $5.45 / 5.98$ & $5.19 / 5.98$ & $5.19 / 5.98$ & $5.45 / 5.98$ & $5.19 / 5.98$ & $5.45 / 5.98$ \\
& 1.0 & $\mathbf{4 . 4 1 / 6 . 2 4}$ & $\mathbf{4 . 4 1 / 6 . 2 4}$ & $\mathbf{4 . 4 1 / 6 . 2 4}$ & $\mathbf{4 . 4 1 / 6 . 2 4}$ & $\mathbf{4 . 4 1 / 6 . 2 4}$ & $\mathbf{4 . 4 1 / 6 . 2 4}$ \\
& 1.5 & $5.19 / \mathbf{6 . 2 4}$ & $5.19 / \mathbf{6 . 2 4}$ & $5.19 / \mathbf{6 . 2 4}$ & $5.19 / \mathbf{6 . 2 4}$ & $5.19 / \mathbf{6 . 2 4}$ & $5.19 / \mathbf{6 . 2 4}$ \\
& 2.0 & $5.71 / \mathbf{6 . 2 4}$ & $5.71 / \mathbf{6 . 2 4}$ & $5.71 / \mathbf{6 . 2 4}$ & $5.71 / \mathbf{6 . 2 4}$ & $5.71 / \mathbf{6 . 2 4}$ & $5.71 / \mathbf{6 . 2 4}$ \\
\hline
\end{tabular}

In our experiments on real data in Chapter 7 we already compared the results obtained with averaging wavelets only to the results obtained when using both averaging and gradient wavelets.

When comparing the results obtained with averaging wavelets only, and the results obtained using both types of wavelets, we may make two observations. First, the detection rates when using averaging wavelets only, are slightly worse than those obtained when using averaging and gradient wavelets. Second, the 
main difference between using averaging wavelets only, and both averaging and gradient wavelets, is in the estimation of the scales of the specific brain regions. The scale estimation is significantly worse when using averaging wavelets only. We conjecture that the reason for this is that gradient wavelets can be informative not only when they perfectly fit a task-specific region (like an averaging wavelet), but also when they do not overlap the entire task-specific region; they can detect the edge of the region in that case.

\subsubsection{Wavelet basis}

Our wavelet basis, as defined in Subsection 6.4.1, is an non-orthogonal basis that is overcomplete in terms of spatial locations. The overcompleteness causes the relevant directions in the feature space to be present in our data. One might argue that in signal-reconstruction applications, it is common to have a complete orthogonal basis (Mallat, 1999), and that we should have used such a basis as well. However, there are two main reasons why we have chosen not to use a complete orthogonal basis.

First, in a complete decomposition, one has only a few wavelets of a large scale, that do not overlap each other. Large-scale wavelets are thus present at a limited number of spatial locations. Using a representation like this, a large specific region that has a spatial extent matching one of the wavelet scales exactly, might not be detected if its location falls in between that of two large-scale wavelets in the complete decomposition. In our overcomplete decomposition, there will be a wavelet covering the specific region exactly, and thus the region will be detected.

Second, although there exist orthogonal decompositions on a triangular or hexagonal grid (Watson and Amahuda, 1989), these decompositions are not Haarlike. This means that feature extraction cannot be performed using our fast $O(1)$ method, and a slow method would result.

\subsubsection{Wavelets and spatial smoothing}

The wavelet features that we employ perform a spatial smoothing over a local region of the cortex. Effectively, this means that vertices are classified based not only on their own time course, but also on time courses of vertices in their local neighbourhood. In addition, the effect of noise is to some degree averaged out. One would thus wonder whether a similar result might be obtained by spatially presmoothing the data, and then using the standard GLM method. In this manner, the spatially smoothed time course of a vertex would also contain information on its neighbours' time courses.

We argue that the use of wavelets in our method has one clear advantage over spatial pre-smoothing of the data: when using wavelets, the "smoothing" scale is data-driven. When pre-smoothing data, one has to choose a scale for the smoothing filter. This choice is problematic. If one chooses the scale too small, the resulting filtered data will not contain a significant amount of neighbourhood information, and noise will not be reduced significantly. If one chooses the scale too large, significant signals in small task-specific regions will be averaged out, 
resulting in a method that is incapable of finding small task-specific regions. In the region-based method, we use wavelets at different scales that are selected based on their specificity. The use of wavelets at different scales means that the scale of smoothing will be inherently adapted to the scale of task-specific activations in a given region. This enables smoothing at different scales in different regions, where the scale of smoothing is automatically determined.

Nonetheless, we recommend that the region-based method's results are compared to those of a GLM in which the data is spatially pre-smoothed at different scales. Such an experiment would enable the quantitative validation of our argument stated above.

\subsection{The use of Bayesian classification}

In our method, we employ a method based on Bayesian statistics to select features and to classify vertices. We exploit spatial-relation information in our feature extraction, and then determine whether a feature should be selected, on that feature's own posterior probabilities only. One might thus regard our method as a thinly veiled massively univariate method similar to, e.g., the GLM. However, there are two essential differences between our method and a classical massively univariate method.

The first and foremost distinction is in the amount of information required by the GLM and by our method. The GLM requires not only the experimental protocol, i.e., when each stimulus was exposed to the subject, but also a model for the BOLD response (Boynton et al., 1996). In contrast, our method requires the experimental protocol only. This distinction is important because the actual BOLD response shows a greater variability than is incorporated in current models. In addition, the current simulation data give an unfair advantage to the GLM, because similar Boynton models are used in both the activation simulation, and in the the GLM predictor calculation.

The second distinction between our method and a massively univariate method, is (1) that our "univariate selection" takes place on the level of features, (2) that each both contain information on multiple vertices, and (3) that influence the specificity-classification of multiple vertices. Therefore our method, when viewed on the vertex-level, is multivariate indeed, and differs per (1) from a massively univariate method.

\subsection{Related work}

The region-based method is related to numerous other recent research in the area of fMRI analysis and neurological imaging in general. In this section, we describe four classes related work, and the relevance of our work in the light of the work in these four categories. First, we discuss related work that, like the region-based method, does take spatial correlation between voxels or vertices into account, but does so in a spatially global manner. Second, we discuss related work that is region based or otherwise based on localised multivariate analysis. Third, we 
discuss work that is based on inherently univariate analysis, but where information on spatial relations is exploited after the initial analysis. Fourth, we discuss work that deals with feature selection.

\subsubsection{Global spatial correlations}

The first class of related work is based on global multivariate (or "massively multivariate") analysis. Massively multivariate methods have been developed to take spatial correlations between voxels into account, in order to detect distributed activation where the correlation between voxels is significant, whereas each voxel by itself would not be significant. Massively multivariate methods attempt to characterise spatially distributed patterns (Buchel and Friston, 1997), by considering spatial "modes" across the entire brain. Examples of massively multivariate techniques are the Generalised Eigenimage Analysis (GEA) / PCA (Friston, 1995; Mourão-Miranda et al., 2005), methods based on Canonical Variate Analysis (CVA) (Buchel and Friston, 1998), and methods based on Independent Component Analysis (ICA) (Formisano et al., 2004). Massively multivariate methods, with the exception of CVA, attempt to cluster activations in an unsupervised manner. That is, they do not use information on the stimulus class. Thus, in contrast to the region-based method, they cannot detect task-specific brain regions. Massively multivariate methods suffer from a problem in this regard. The large number of voxels $\left(\sim 10^{4}\right.$ to $\left.10^{5}\right)$, combined with a small number of temporal instances $\left(\sim 10^{1}\right.$ to $\left.10^{2}\right)$, makes the reliable estimation of spatial modes problematic.

\subsubsection{Localised multivariate analysis}

The second class of related work is based on localised multivariate analysis. There are a number of methods that, like ours, exploit the spatial relations between neighbouring vertices or voxels, in a multivariate manner. The method by Aston et al. (2006) works on volume data, i.e., time courses for every voxel in the brain volume. Their method extracts 3-dimensional wavelets, and then performs a GLM analysis in wavelet space. Subsequently, a thresholding is performed, based on the wavelets' specificity, and finally the wavelet specificities are transformed back to voxel space. With this method, we share the property of using wavelets as filters, where the scale of filtering is data driven. In addition, we share the property of feature thresholding based on specificity. Unlike the method by Aston et al., we employ the specificity of wavelets in order to make a specific-region scale estimate. Also unlike their method, we employ an overcomplete wavelet decomposition. This allows us more flexibility in the position of, in particular, large wavelets.

Additionally, a very interesting recent method by Takerkart and Daubechies (2006) extends the idea of Aston et al., by explicitly exploiting not only the spatial relation, but also the scale relation between wavelets. That is, if a large wavelet is already sufficiently specific then it stands to reason that smaller wavelets, within the region covered by the large specific wavelet, are specific too. In that case,

the threshold for specificity for these smaller wavelets is lowered, so that a more 
detailed spatial outline of the specific region may be obtained. Takerkart and Daubechies do not obtain a scale estimate for task-specific regions, which the region-based method does do. Yet, we still recommend to integrate a similar scale-relation exploitation into the region-based method as well.

Subsequently, there is the recent work by Carroll et al. (2006). Like in our method, they exploit the information present in spatial relationships. To this end, they use an overcomplete wavelet basis similar to that used in Viola and Jones (2001b), and employ AdABoost (Freund and Shapire, 1996) to select features and to train a classifier. However, in contrast to the region-based method, Carroll et al. do not obtain a scale estimate for task-specific regions. Given the high dimensionality of the feature space in both our method and theirs, the use of AdABoost may be a viable future extension of our method.

Finally, there is the searchlight approach by Kriegeskorte et al. (2006). They compare the performance of a Gaussian naive Bayes classifier based on either separate voxels, or on $3 \times 3 \times 3$-voxel "searchlight" volumes. They conclude that the use of information from a larger spatial area improves brain-state classification performance. Their method can be regarded as a simple version of our method, where only averaging wavelets of a single scale are used.

\subsubsection{Univariate analysis and spatial relations}

The third class of related work starts out by doing a univariate analysis, after which spatial information is used to improve the specific-region results. Weeda et al. (2006) first use a conventional voxel-selection method, after which an EM clustering algorithm (Verbeek et al., 2003) is used to determine the number of active areas, and their approximate extent. This clustering could be used in our method to aid in the automatic detection of the number of active areas. However, in our method, a modified EM algorithm that uses the additional scale-information of our method, could be employed. The work by Vanello et al. (2006) uses a clustering technique similar to Weeda et al.. Both works do use a mechanism to estimate the scale of a task-specific region, but our method is superior in that it integrates the notion of scale into the learning algorithm itself. It is clear from the result that this improves the detection of small regions such as the FFA.

\subsubsection{Feature selection}

The fourth class of related work deals with the problem of feature selection. Due to the high dimensionality of the feature space, both our method, and many others, have to select an informative fraction of features. We select features based on their specificity, given the posterior probabilities $p\left(c_{i} \mid v_{j}\right)$ obtained after Bayesian training. Some of the work referenced above deals with feature selection: Aston, Turkheimer, and Brett (2006) select features based on their GLM $\beta$ value, and Takerkart and Daubechies (2006) extend this selection method by taking scale relations into account. Carroll et al. (2006) use the combined training/selection method inherent to AdABoost, while Pereira et al. (2006) use a specificity measure similar to ours. Duchesnay et al. (2006) describe a voxel-based method. 
However, they rank voxels using a one-sides ANOVA, and subsequently select voxels having a smaller than $10 \%$ false discovery rate (Genovese et al., 2002) as possible features. Particularly, the FDR-based selection procedure can be readily adapted to select wavelets in our method. This might provide our method with a statistically better founded feature-selection paradigm. Finally, Cox and Savoy (2003) use an ANOVA method to preselect voxels for classification.

Mitchell et al. (2004) describe a Gaussian naive Bayesian method for brainstate classification. They regard selection strategies based both on the selection of the, say, $n$ discriminating voxels, and of the $n$ voxels that are most discriminating with respect to the rest condition. For brain-state classification, they find that the latter yields slightly better results. However, Mitchell et al. do not attempt to create spatial brain-specificity maps, and work in a univariate manner. Yet, we believe that for our application, the selection of discriminating voxels is to be preferred, for the following two reasons. First, our primary goal is not brain-state classification, but rather the creation of a brain-specificity map. Therefore, the areas of most interest to us are those that are most specific, regardless of whether they are also the best to predict the brain state. Second, our method operates on wavelets, which provide more spatial averaging than single voxels. Therefore, the probability of a feature being specific due to random effects is smaller than in the case voxels. Nonetheless, Mitchell et al.'s idea of looking only at features that differ sufficiently from the resting-state condition is an interesting one. This idea can easily be integrated into our method, by first training a classifier that contrasts between the active (either one of the two stimuli) state and the resting state, and using the log-likelihood values from this contrast as priors for our feature selection.

\subsection{Future directions}

In this section we explore future extensions and application areas for the regionbased method. In Subsection 8.5.1, we discuss a possible improvement in the region-method's scale-estimation mechanism. In Subsection 8.5.2 we propose an extension to the classification of more than two stimuli. In Subsection 8.5.3, we discuss the extension of the region-based method to cortical quantities other than fMRI signals.

\subsubsection{Fully automated scale estimation}

It should be remarked that in the scale-estimation experiments, we have assumed the centre of the specific regions to be known. Of course, in an experiment based on actually measured activation data, one does not know the exact location and boundaries of a specific region. Therefore, although our scale-estimation results are good, it requires an experimenter to indicate the centre of the task-specific region. We performed preliminary experiments in which we attempt to determine automatically the centre of a specific region by choosing that vertex for which the specificity value within the region is highest. Typically, for higher CNARs (larger than 0.7), we see that the cluster centre is estimated to be within two grid units from the actual location. Scale estimates do become worse than in the 
previously shown tables, but still reflect the fact that there is a scale difference between regions in the brain with unequally sized regions.

\subsubsection{Extensions to multi-stimulus classification}

In this chapter, we examined a framework based on Bayesian statistics that identifies stimulus-specific regions in the brain based on the time signals across regions of the brain's cortex neighbouring the voxel of interest. In our method, we distinguish between $n=2$ stimuli. In this section, we explore the possibilities for expanding our method to a multi-stimulus environment $(n>2)$.

The first thing to note, is that in order to apply voxel classification or naiveBayesian brain-state classification, the method does not have to change. To see this, note that for $n$ classes, we may calculate

$$
\begin{aligned}
& P\left(a_{i} \mid c_{1}\right) \\
& P\left(a_{i} \mid c_{2}\right) \\
& \vdots \\
& P\left(a_{i} \mid c_{n}\right)
\end{aligned}
$$

from the MTC data without modifications over the 2-class case. This is performed for all $n$ stimuli, for all features, and for all possible values of the features (in our case either "true" of "false"). A full classification of each voxel may then be obtained as

$$
\begin{aligned}
P\left(v_{j} \text { specific to } c_{1}\right) & \propto \prod_{i \text { overlapping voxel } j} P\left(a_{i} \mid c_{1}\right) \\
P\left(v_{j} \text { specific to } c_{2}\right) & \propto \prod_{i \text { overlapping voxel } j} P\left(a_{i} \mid c_{2}\right) \\
\vdots & \\
P\left(v_{j} \text { specific to } c_{n}\right) & \propto \prod_{i \text { overlapping voxel } j} P\left(a_{i} \mid c_{n}\right) .
\end{aligned}
$$

The same is true for a naive-Bayesian brain-state classification. The main difficulty in applying our method lies in the selection of a fraction of features. In our original 2-class method, we use only features having a sufficiently large absolute log-likelihood value. As we now work with more than 2 classes, a loglikelihood is not defined. We therefore propose to select and group features based on their Shannon entropy $H(i)$. That is, we would like to select features that have a posterior-probability function over the classes $j$ that is as strongly "peaked", or discriminating, as possible:

$$
H(i)=-\sum_{j=1}^{n} P\left(a_{i} \mid c_{j}\right) \log _{2} P\left(a_{i} \mid c_{j}\right)
$$


Based on these values, it is possible to group features having a similar or equal entropy together. Then, one takes only the groups with a sufficiently low entropy into account.

We recommend the extension of our method to allow for multi-stimulus classification in a manner similar to that outlined above.

\subsubsection{Application to other cortical-surface quantities}

In the Chapters 6 to 8, our region-based method was successfully applied to the analysis of a fMRI BOLD signal on the cortical surface. We expect that the method can be applied to other cortical-surface quantities as well, albeit with some modifications. This expectation arises from the fact that our method requires only a very small amount of information to perform its analysis. Effectively, it distinguishes between two classes, based solely on a number of samples for each class, where the samples consist of wavelet features that can be discretised ${ }^{1}$. It should be noted that the region-based method depends on the interpretation of neither the discretised feature values, nor the classes.

Therefore, we expect that our method can be used on, e.g., cortical-curvature data. For example, our method could identify cortical regions of which the curvature differs between Alzheimer patients and healthy subjects. In this case, our method needs aligned curvature data of a group of Alzheimer patients, and a group of healthy patients. Wavelet features can be extracted from each of the cortices in the same manner as in our fMRI experiments, although now, one sample is extracted from the curvature-data of each patient. Feature values can subsequently be discretised into, e.g., "above-average curvature" and "below-average curvature" values. Classes can be arbitrarily assigned, e.g., Alzheimer patients are assigned $c_{1}$, and healthy subjects are assigned $c_{2}$. At this point, our method can be run with no more modifications, and will yield a brain map showing where cortical-curvature differs most between Alzheimer patients and healthy subjects.

We recommend extending the method to allow the study of cortical-curvature differences between groups of subjects. In a more general sense, any property that can be measured on the cortical surface, and that is expected to differ between two classes of samples - be it stimulus classes, classes of test subjects, or otherwiseforms a possible dataset to which our method could be applied.

\footnotetext{
${ }^{1}$ In the region-based method we discretise each feature into one of two values. However, there is no inherent limitation in using more than two possible values.
} 


\section{General conclusions and recommendations}





\section{Chapter 9}

\section{Conclusions}

In this thesis we investigated the use of spatial-context information in order to improve the performance of machine-learning based image-analysis algorithms. We developed context-based methods for an application in the cultural-heritage domain, and an application in the medical domain.

In the cultural-heritage domain, we implemented two classes of context-based methods to improve the performance of object detection: the context-detection method and the gradient method. In the medical domain, we developed the regionbased method to improve the performance of functional MRI brain mapping.

The outline of this final chapter is as follows. In Section 9.1 we answer the research questions posed in Section 1.3 for both application domains. Furthermore, we summarise the main conclusions of the individual chapters. In Section 9.2, we return to the overall problem statement that we originally introduced in Section 1.2. Finally, in Section 9.3, we present directions for future research.

\subsection{Answers to the research questions}

In Section 1.3 we posed two domain-specific problem statements and six research questions for the two application domains. In this section, we will answer the corresponding research questions and the domain-specific problem statements.

\subsubsection{The cultural-heritage domain}

For the cultural-heritage domain, we posed the following problem statement.

Domain-specific problem statement: To what extent can the local spatial context of an object enhance the reliability of object detection in natural and painted images?

Starting from this problem statement, we formulated the two research questions RQ1 and RQ2 in Section 1.3. Moreover, during the research, we augmented RQ1 with the two additional research questions RQ1a and RQ1b. In total, we have four research questions for the cultural-heritage domain. 
Research question 1 (RQ1): How can local spatial context be used to lower the number of false detections (i.e., achieve a more accurate detection) at an acceptable speed in automatic object detection?

Research question 1a (RQ1a): To what extent can knowledge of relative locations within the context help lower the false-detection rate?

Research question 1b (RQ1b): What classifier design and feature sets are optimal for the gradient method?

Research question 2 (RQ2): What size of the context region is best suited to lower the false-detection rate?

Before addressing these research questions, we provide a summarise of the main conclusions of Chapters 3, 4, and 5. Then, we give four answers in the form of conclusions.

\section{The context-detection method}

The results in Chapter 3 show a clear trade-off between detection speed and detection accuracy. The use of context detection for object detection yields a reduction of the search space for the object detector, and thus allows for faster object detection. However, this increased speed comes at the price of a reduction in detection accuracy.

As such, even though the context-detection method brings about a considerable increase in detection speed, it does not solve the primary research goal of reducing the number of false positives. We assume that this is due to the large intra-class variance of the large context regions, combined with the large number of possible features. This observation inspired us to develop the gradient method, and to pose the research questions RQ1a and RQ1b.

\section{The gradient method}

Our main motivation for introducing the gradient method was to reduce the dimensionality of the learning problem by taking domain knowledge - in this case the relative location of small parts with respect to the object - into account.

The results in Chapter 4 indicate that the gradient method succeeds in considerably lowering the number of false positives at a given detection rate. In Chapter 5, we addressed the question: is the increased performance purely due to the fact that we guide the learning process using knowledge on the spatial relationship within the context? From the experimental results, we may conclude that the improvement in object-detection performance by the gradient method is indeed due to the fact that knowledge on relative locations allows a sensible feature selection. 


\section{Four conclusions}

Regarding research question RQ1, we may conclude that the use of context is beneficial in improving the object-detection performance. In particular, the amount of false positives at a given detection rate can be lowered substantially in facedetection experiments.

Furthermore, with regard to research question RQ1a, we may conclude that the beneficial effect of using context is mainly caused by the fact that our algorithm uses information on the spatial relations between the object and the different parts of its context, in order to lower the dimensionality of the feature space.

Concerning research question RQ1b, we found a good match of features and classifiers, and described reasons why this combination works well. We are pleased with the result but must conclude that no optimality can be guaranteed.

Finally, with regard to research question RQ2, we may conclude that there exists an optimal size of the local-context region, from which to draw information. In our face-detection experiments, a region about twice as large as the face, was found to yield the best results.

\subsubsection{The medical domain}

For the medical domain, we posed the following domain-specific problem statement.

Domain-specific problem statement: To what extent can local context enhance the performance of detecting task-specific regions of activity in the brain?

From this problem statement, we formulated the four research questions RQ3 to RQ6 in Section 1.3. During the research, we augmented this set of four research questions with an additional research question RQ4a. In total, we have the following five research questions for the medical domain.

Research question 3 (RQ3): Can a new region-based method detect brain regions with high specificity on a level comparable to or better than the standard method used in the field?

Research question 4 (RQ4): Can a new method deliver a reliable scale estimate for specific regions?

Research question 4a (RQ4a): Can a new method effectively deal with classification in a very high-dimensional feature space? 
Research question 5 (RQ5): Does the applicability of the new method generalise from simulated activation data to real data?

Research question 6 (RQ6): Can the region-based method cope with interpersonal differences in anatomy in a better way than standard methods?

Before addressing these research questions, we provide a summary of the main conclusions of the Chapters 6, 7, and 8. Then, we give five answers in the form of conclusions.

\section{The region-based method}

Research question RQ3 is whether our method would yield a specific-region detection comparable to, or better than, that of traditional methods. In order to answer this question, we compared our method against the GLM in an extensive range of experimental conditions on both simulated and real fMRI data. From the results, we may conclude that our method performs comparably for higher contrast-tonoise ratios (CNARs), and clearly outperforms the GLM for low CNARs. We explain the good performance of our method for lower CNARs by the fact that our method effectively reduce the noise influence by applying a region-specific spatial smoothing.

Research question RQ4 is whether our method would enable a reliable estimation of the scale of specific brain regions. From our results, we may conclude that our method is capable of estimating the scale of specific regions in both simulated and real fMRI data. However, (1) the selection of a proper fraction of features, and (2) the selection of a specific region's exact location remain open problems.

Regarding research question RQ5, the results of Chapter 7 lead us to conclude that our method generalises well from simulated data to real data. This generalisation property is important, given the limitations of simulated data, as set forth in Section 6.7.

With regard to research question RQ6, our method is significantly more robust to inter-subject anatomical differences than the standard vertex-based GLM. In particular, our method is capable of finding small specific brain areas, such as the fusiform face area, despite anatomical differences of which the lateral dimensions are in the same order to magnitude as the size of the specific region. The robustness of our method to inter-subject anatomical differences is made even more apparent by the fact that it performs well even without using specialised cortical alignment. To the best of our knowledge, our method is unique in this respect. Our method is robust to inter-subject anatomical differences because of two reasons. First, our method is multivariable, and uses features that are both spatially extended, and take spatial-relation information into account. This allows for the proper identification of regions despite significant differences in the regions' location among subjects. Second, our method employs an efficient heuristic on Bayesian statistics, that excludes a great deal of noisy data. This heuristic also provides a way to enable learning with a high dimensionality of the feature space, thus providing an answer to research question RQ4a. 


\section{Five conclusions}

Concerning the medical domain, we may conclude that the use of context is beneficial in improving the brain-mapping performance. In particular, in relation to the research questions posed above, we may conclude the following.

First, with regard to research question RQ3, our region-based method outperforms the standard method in the field of detecting regions with high specificity, in both simulated and real data. This means that at a given false-detection rate, our method detects a higher percentage of the task-specific brain areas.

Second, the efficient heuristics on Bayesian statistics developed within the framework of our method, adequately addresses the research question RQ4a of learning with data in a high-dimensional feature space.

Third, with regard to research question RQ4, the region-based method is indeed capable of delivering a reliable scale estimate for task-specific regions. This has been verified on both simulated and real data.

Fourth, regarding research question RQ5, the region-based method's performance generalises well from simulated data to real data.

Fifth, concerning research question RQ6, our method has been shown to cope better with interpersonal differences than the standard method used in the field.

\subsection{Answer to the problem statement}

In Section 1.2, we formulated the main problem statement of this thesis:

Problem statement: How can information from a local spatial context be utilised to improve the performance or reliability of imageanalysis techniques?

Below, we provide a brief answer to the problem statement based on the research described in this thesis. In the two application domains investigated, the use of information from the local spatial context has proven to be beneficial in improving the performance. In particular, the use of knowledge on spatial relations within the local context has proven to be the key factor. In both application domains, the use of this information has proven to reduce the dimensionality of the learning problem, which makes it more tractable from a machine-learning point of view. This specific manner of reducing the dimensionality does retain important information from the context.

In images, the local spatial context contains information that facilitate the detection of objects. The information results from the statistics of natural images or fMRI images. Unfortunately, the statistics vary with the application domain and may be specific to the task under consideration. Therefore, methods that exploit contextual information have to be tailored to the domain and task at hand. Once the domain and task specific knowledge has been identified and incorporated 
into the detection algorithm, considerable improvements in the performance and reliability of image analysis can be obtained.

\subsection{Two directions for future research}

In this section, we propose two directions for future research, based on the insights obtained in this thesis.

Our first proposal is to investigate how spatial context can be applied to other image-analysis domains. Context is used to some degree in other analysis methods, e.g., it is used in ecology (Borcard and Legendre, 2002), in political analysis (Ward and Gleditsch, 2002). and in remote sensing applications (Price, 1990). We believe that there exist many other analysis domains in which context may prove to be a rich source of additional information, and that the use spatial context as used in existing context-based methods can be extented.

Our second proposal is to investigate how spatial context can be used in imageanalysis systems in a more general manner than described in the thesis. In our study, we confined ourselves to the use of local context, and concluded that the main contributing factor to a better performance stems from the use of spatialrelation information within that local context. The use of context in a more general sense can be regarded in two ways. First, the use of spatially extended, or global context can be investigated. Initial research in this direction, e.g., Torralba and Sinha (2003), Torralba et al. (2006), indicates the potential of using global context for object detection and for guiding attention. We propose to investigate how local and global context can be integrated to facilitate image analysis. Second, the use of context can be further broadened by including other modalities. In the cultural-heritage domain, the additional modalities may consist of text, video, and sound. In the medical domain, the additional modalities include coregistered MRI, EEG, and MEG data. However, in the general medical domain, the range of modalities may be wider, since the patient — as a human being, rather than as an "image" or "object" - is a rich source of information. This means that in addition to physically measured quantities such as coregistered MRI, EEG, and MEG data, more loosely structured information such as that from questionnaires, or from the patient record, may be of use in classification. We believe that in tasks such as brain mapping, charactarized by the fact that the activities cannot be detected by means other than the technical methods such as fMRI and EEG, this more loosely structured information may not be of much use. However, in more general medical classification tasks, the use of patient-centred and loosely structured information should be considered. 


\section{References}

Abragam, A. and Hebel, L. C. (1961). The Principles of Nuclear Magnetism. American Journal of Physics, Vol. 29, No. 12, pp. 860-861. ISSN 0002-9505. [69]

Agarwal, S. (2002). Learning a Sparse Representation for Object Detection. Proceedings of the Seventh European Conference on Computer Vision (eds. A. Heyden, G. Sparr, M. Nielsen, and P. Johansen), Copenhagen, Denmark. ISSN 0302-9743. [61]

Ahn, L. von and Dabbish, L. (2004). Labeling images with a computer game. CHI '04: Proceedings of the SIGCHI conference on Human factors in computing systems, pp. 319-326, ACM Press, Vienna, Austria. ISBN 1-58113-702-8. [63]

Ahn, L. von, Liu, R., and Blum, M. (2006). Peekaboom: a game for locating objects in images. CHI '06: Proceedings of the SIGCHI conference on Human Factors in computing systems, pp. 55-64, ACM Press, Montréal, Québec, Canada. ISBN 1-59593-372-7. [63]

Aston, J. A. D., Turkheimer, F. E., and Brett, M. (2006). HBM Functional Imaging Analysis Contest Data Analysis in Wavelet Space. Human Brain Mapping, Vol. 27, No. 5, pp. 372-379. ISSN 1065-9471.[126, 127]

Baddeley, R. J. and Hancock, P. J. B. (1992). Principal Components of Natural Images. Network: computation in neural systems, Vol. 3, pp. 61-70. ISSN 0954-898X. [2, 47]

Bashir, F. and Porikli, F. (2006). Performance Evaluation of Object Detection and Tracking Systems. IEEE International Workshop on Performance Evaluation of Tracking and Surveillance (PETS) (ed. J. M. Ferryman), New York, NY. ISBN 0-7049-1422-0. [1]

Bellman, R. E. (1972). Adaptive control processes : a guided tour. Princeton University Press, Princeton, NJ, 5th edition. ISBN 0-691-07901-3. [24]

Bergboer, N. H., Postma, E. O., and Herik, H. J. van den (2003). ContextEnhanced Object Detection in Natural Images. Proceedings of the BNAIC 2003 (eds. T. Heskes, P. Lucas, L. Vuurpijl, and W. Wiegerinck), pp. 27-34, Nijmegen, The Netherlands. [-] 
Bergboer, N. H., Postma, E. O., and Herik, H. J. van den (2004). A ContextBased Model of Attention. Proceedings of the 16th European Conference on Artificial Intelligence, ECAI 2004 (eds. R. López de Mánteras and L. Saitta), pp. 927-931, IOS Press, Valencia, Spain. ISSN 0922-6389. [-]

Bergboer, N. H., Postma, E. O., and Herik, H. J. van den (2005). Visual object detection for the cultural heritage. Proceedings of the XVI international conference of the Association for History and Computing (AHC2005), pp. 33-38, Royal Netherlands Academy of Arts and Sciences, Amsterdam, The Netherlands. ISBN 90-6984-456-7. [-]

Bergboer, N. H., Postma, E. O., and Herik, H. J. van den (2006a). Accuracy versus Speed in Context-Based Object Detection. Pattern Recognition Letters, Vol. 28, No. 6, pp. 686-694. ISSN 0167-8655. [-]

Bergboer, N. H., Postma, E. O., and Herik, H. J. van den (2006b). Context-Based Object Detection in Still Images. Image and Vision Computing, Vol. 24, No. 9, pp. 987-1000. ISSN 0262-8856. [-]

Biederman, I. (1981). On the semantics of a glance at a scene. Perceptual organization (eds. M. Kubovy and J. Pomerantz). Erlbaum, Hillsdale, NJ. [12, $25]$

Bileschi, S. and Wolf, L. (2005). A Unified System for Object Detection, Texture Recognition and Context Analysis Based on the Standard Model Feature Set. Proceedings of the British Machine Vision Conference (eds. W. F. Clocksin, A. W. Fitzgibbon, and P. H. S. Torr), Alden Group Ltd, Osney Mead, Oxford, UK. ISBN 1-901725-29-4. [62]

Borcard, D. and Legendre, P. (2002). All-scale spatial analysis of ecological data by means of principal component analysis of neighbour matrices. Ecological Modelling, Vol. 153, pp. 51-68. [138]

Boynton, G. M., Engel, S. A., Glover, G. H., and Heeger, D. J. (1996). Linear Systems Analysis of Functional Magnetic Resonance Imaging. The Journal of Neuroscience, Vol. 16, No. 13, pp. 4207-4221. ISSN 1529-2401. [83, 95, 99, $125]$

Buchel, C. and Friston, K. J. (1997). Characterizing functional integration, pp. 127-140. Academic Press USA, New York, NY. ISBN 0-12-264841-2. [126]

Buchel, C. and Friston, K. J. (1998). Dynamic changes in effective connectivity chracterized by variable parameter regression and Kalman filtering. Human Brain Mapping, Vol. 6, No. 5, pp. 403-408. ISSN 1065-9471.[126]

Carbonetto, P., Freitas, N. de, and Barnard, K. (2004). A Statistical Model for General Object Recognition. Proceedings of the European Conference on Computer Vision (ICCV), Lecture Notes in Computer Science, SpringerVerlag. ISSN 0302-9743. [62] 
Carroll, M. K., Norman, K. A., Haxby, J. V., and Schapire, R. E. (2006). Exploiting Spatial Information to Improve fMRI Pattern Classification. Poster at the 12th Annual Meeting of the Organization for Human Brain Mapping. [127]

Cawley, G. C. (2000). MATLAB Support Vector Machine Toolbox (v0.50 $\beta$ ) (http://theoval.sys.uea.ac.uk/ gcc/svm/toolbox). University of East Anglia, School of Information Systems, Norwich, Norfolk, UK. [17]

Chen, X. and Strother, S. C. (2006). Testing fMRI Signal Detection: Dependence on ROC Technique and Simulation Structure. Poster at the 12th Annual Meeting of the Organization for Human Brain Mapping. [95, 99]

Cox, D. D. and Savoy, R. L. (2003). Functional magnetic resonance imaging (fMRI) "brain reading": detecting and classifying distributed patterns of fMRI activity in human visual cortex. NeuroImage, Vol. 19, pp. 261-270. ISSN 1053-8119. [128]

Dantzig, M. M. van (1973). Pictology. An analytical method for attribution and evaluation of pictures. E. J. Brill, Leiden, The Netherlands. ISBN 900403662-8. [60]

Domingos, P. and Pazzani, M. (1996). Beyond Independence: Conditions for the Optimality of the Simple Bayesian Classifier. Proceedings of the International Conference on Machine Learning, pp. 105-112. ISBN 1-55860-419-7. [78]

Dorkó, G. and Schmid, C. (2004). Object Class Recognition Using Discriminative Local Features. Submitted to IEEE Transactions on Pattern Analysis and Machine Intelligence. [61]

Duchesnay, E., Thirion, B., Poline, J.-B., Lebihan, D., and Dehaene, S. (2006). Predicting stimuli from visual cortex activation using voxels selection and SVM classifier. Poster at the 12th Annual Meeting of the Organization for Human Brain Mapping. [127]

Duda, R. O., Hart, P. E., and Stork, D. G. (2001). Pattern Classification. John Wiley \& Sons, Inc., New York, NY. ISBN 0-471-05669-3. [35]

Eriksen, C. W. and James, J. S. (1986). Visual Attention Within and Around the Focus of Attention. Perception and Psychophysics, Vol. 40, No. 4, pp. 225-240. ISSN 1532-5962. [13]

Eriksen, C. W. and Yeh, Y. Y. (1985). Allocation of Attention in the Visual Field. Journal of Experimental Psychology: Human Perception and Performance, Vol. 11, No. 5, pp. 583-597. ISSN 0272-4987.[13]

Fei-Fei, L., Fergus, R., and Perona, P. (2004). Learning Generative Visual Models from Few Training Examples: An Incremental Bayesian Approach Tested on 101 Object Categories. CVPR Workshop of Generative Model Based Vision. [61] 
Fergus, R., Perona, P., and Zisserman, A. (2003). Object Class Recognition by Unsupervised Scale-Invariant Learning. IEEE Computer Society Conference on Computer Vision and Pattern Recognition (CVPR), Vol. 2, pp. 264-271. $[61]$

Fergus, R., Fei-Fei, L., Perona, P., and Zisserman, A. (2005). Learning Object Categories from Google's Image Search. Proceedings of the Tenth IEEE International Conference on Computer Vision (ICCV'05). ISSN 1550-5499. [64]

Forman, S. D., Cohen, J. D., M, M. Fitzgerald, Eddy, W. F., Mintun, M. A., and Noll, D. C. (1995). Improved assessment of significant activation in functional magnetic resonance imaging (fMRI): use of a cluster-size threshold. Magnetic Resonance Imaging, Vol. 33, No. 5, pp. 636-647. ISSN 0730-725X. [2]

Formisano, E., Esposito, F., Salle, F. di, and Goebel, R. (2004). Cortex-based independent component analysis of fMRI time series. Magnetic Resonance Imaging, Vol. 22, pp. 1493-1504. ISSN 0730-725X. [126]

Freeden, W. and Windheuser, U. (1996). Spherical wavelet transform and its discretization. Advances in Computational Mathematics, Vol. 5, No. 1, pp. 51-94. ISSN 1019-7168. [75]

Freund, Y. and Shapire, R.E. (1996). Experiments with a new boosting algorithm. Proceedings of the Thirteenth International Conference on Machine Learning (ed. L. Saitta), pp. 148-156, Bari, Italy. ISBN 1-55860-419-7. [17, $127]$

Friston, K. J. (1995). Functional and effective connectivity in neuroimaging: A synthesis. Human Brain Mapping, Vol. 2, pp. 56-78. ISSN 1065-9471. [126]

Genovese, C. R., Lazar, N. A., and Nichols, T. E. (2002). Thresholding of Statistical Maps in Functional Neuroimaging Using the False Discovery Rate. NeuroImage, Vol. 15, pp. 870-878. ISSN 1053-8119. [86, 128]

Gershenfeld, N. (1999). The Nature of Mathematical Modeling. Cambridge University Press, Cambridge, MA. ISBN 0-521-57095-6. [31, 36]

Gyorfi, L., Devroye, L., and Lugosi, G. (1996). A Probabilistic Theory of Pattern Recognition. Springer-Verlag, New York, NY. ISBN 0-387-94618-7. [44]

Haar Romeny, B. ter and Florack, L. M. J. (2000). Front-End Vision: a Multiscale Geometry Engine. Proceedings of the IEEE International Workshop on Biologically Motivated Computer Vision, Lecture Notes in Computer Science, Springer-Verlag, Heidelberg, Germany. Seoul, Korea. [30]

Halgren, E., Dale, A. M., Sereno, M. I., Tootell, R. B. H., Marinkovic, K., and Rosen, B. R. (1999). Location of Human Face-Selective Cortex With Respect to Retinotopic Areas. Human Brain Mapping, Vol. 7, pp. 29-37. ISSN 10659471. [100, 105] 
Haxby, J. V., Ungerleider, L. G., Horowitz, B., Maisog, J. Ma., Rapoport, S. I., and Grady, C. L. (1996). Face encoding and recognition in the human brain. Proceedings of the National Academy of Sciences, Vol. 93, pp. 922-927. ISSN 1091-6490. [100, 105, 116]

Haxby, J. V., Gobbini, M. Ida, Furey, M. L., Ishai, A., Schouten, J. L., and Pietrini, P. (2001). Distributed and Overlapping Representations of Faces and Objects in Ventrel Temporal Cortex. Science, Vol. 293, pp. 2425-2430. ISSN 0036-8075. [100, 105]

Heisele, B., Ho, P., Wu, J., and Poggio, T. (2003). Face recognition: componentbased versus global approaches. Computer Vision and Image Understanding, Vol. 91, pp. 6-19. ISSN 1077-3142. [62]

Helmer, S. and Lowe, D. G. (2004). Object Class Recognition with Many Local Features. Proceedings of the 2004 Conference on Computer Vision and Pattern Recognition Workshop (CVPRW'04), Vol. 12, pp. 187-194. [61]

Hidalgo-Soleto, B., Oliva, A., and Torralba, A. (2005). Human Learning of Contextual Priors for Object Search: Where does the time go? Proceedings of the IEEE Computer Society Conference on Computer Vision and Pattern Recognition, Vol. 3. ISBN 0-7695-2372-2. [63]

Hjelmås, E. and Low, B. K. (2001). Face Detection: A Survey. Computer Vision and Image Understanding, Vol. 83, No. 3, pp. 236-274. ISSN 1077-3142. [1, $11,12,14]$

Holmes, A., Poline, J.-B., and Friston, K. J. (1997). Characterizing brain images with the general linear model, pp. 59-84. Academic Press, New York, NY. [1, $68,86]$

Humphreys, G. W. and Bruce, V. (1989). Visual Attention, Chapter 5. Erlbaum, Hillsdale, NJ. ISBN 0-86377-125-4. [13]

Hunton, D. L., Miezin, F.M., Buckner, R. L., Mier, H. I. van, Raichle, M. E., and Petersen, S.E. (1996). An assessment of functional-anatomical variability in neuroimaging studies. Human Brain Mapping, Vol. 4, No. 2, pp. 122-139. ISSN 1065-9471. [113]

Jain, A. K., Duin, R. P. W., and Mao, J. (2000). Statistical Pattern Recognition: A Review. IEEE Transactions on Pattern Analysis and Machine Intelligence, Vol. 22, No. 1, pp. 4-37. ISSN 0018-9340.[1]

Kanwisher, N., McDermott, J., and Chun, M. M. (1997). The Fusiform Face Area: A Module in Human Extrastriate Cortex Specialized for Face Perception. Journal of Neuroscience, Vol. 17, No. 11, pp. 4302-4311. ISSN 0020-6474. [100]

Knuth, D. E. (1998). The Art of Computer Programming, Volume 3: Sorting and Searching. Addison-Wesley, Reading, MA, 2nd edition. ISBN 0-201-89685-0. $[157]$ 
Koch, C. and Ullman, S. (1985). Shifts in Selective Visual Attention: Towards the Underlying Neural Circuitry. Human Neurobiology, Vol. 4, pp. 35-41. ISSN 0721-9075. [13]

Kriegeskorte, N., Goebel, R., and Bandettini, P. (2006). Information-based functional brain imaging. Proceedings of the National Academy of Sciences, Vol. 103, No. 10, pp. 3863-3868. ISSN 1091-6490. [127]

Kruppa, H., Santana, M. C., and Schiele, B. (2003). Fast and Robust Face Finding via Local Context. Proceedings of the Joint IEEE International Workshop on Visual Surveillance and Performance Evaluation of Tracking and Surveillance, Nice, France. [25, 29, 60]

Kwong, K. K., Belliveau, J. W., Chesler, D. A., Goldberg, I. E., Weisskoff, R. M., Poncelet, B. P., Kennedy, D. N., Hoppel, B. E., Cohen, M. S., Turner, R., Cheng, H.-M., Brady, T. J., and Rosen, B. R. (1992). Dyanamic magnetic resonance imaging of human brain activity. Proceedings of the National Academy of Sciences, Vol. 89, pp. 5675-5679. ISSN 1091-6490. [100, 102]

Lange, O., Meyer-Bäse, A., and Wismüller, A. (2006). Performance evaluation based on cluster validity indices in medical imaging. Proceedings of SPIE (ed. H. H. Szu), Vol. 6247, pp. 404-412, Society of photo-optical instrumentation engineers. ISSN 0277-786X. [1]

Lazebnik, S., Schmid, C, and Ponce, J. (2004). Semi-Local Affine Parts for Object Recognition. Proceedings of the British Machine Vision Conference 2004, Vol. 2, pp. 959-968, London, United Kingdom. ISBN 1-901725-25-1. [61]

Levi, K. and Weiss, Y. (2004). Learning Object Detection from a Small Number of Examples: the Importance of Good Features. Proceedings of the 2004 IEEE Computer Society Conference on Computer Vision and Pattern Recognition CVPR'04. ISBN 0-7695-2158-4. [63]

Lewis, M. B. and Edmonds, A. J. (2003). Face detection: Mapping human performance. Perception, Vol. 32, pp. 903-920. ISSN 1468-4233. [13, 19, 26]

Lienhart, R., Liang, L., and Kuranov, A. (2002). An extended set of haar-like features for rapid object detection. Technical report, Intel Research. [18, 22, $39,56,151]$

Lillesand, T. M., Kiefer, R. W., and Chipman, J. W. (2004). Remote sensing and image interpretation. John Wiley \& Sons, Inc., New York, NY, 5th edition. ISBN 0-471-15227-7. [1]

Logothetis, N. K. and Pfeuffer, J. (2004). On the nature of the BOLD fMRI contrast mechanism. Magnetic Resonance Imaging, Vol. 22, pp. 1517-1531. ISSN 0730-725X. [70]

Mallat, S. (1999). A Wavelet Tour of Signal Processing. Academic Press, New York, NY, 2nd edition. ISBN 0-12-466606-X. [75, 124, 157] 
Martinez-Trinidad, J. F., Ochoa, J. A. Carrasco, and Kittler, J. (eds.) (2006). Progress in Pattern Recognition, Image Analysis and Applications: 11th Iberoamerican Congress on Pattern Recognition, Ciarp 2006, Vol. 4225 of LCNS, New York, NY. Springer-Verlag. ISBN 3-54046556-1. [1]

Martinez, A. M. and Benavente, R. (1998). The AR Face Database. Technical Report CVC \#24, Electrical and Computer Engineering, Purdue University, West Lafayette, IN. [15]

Mikolajczyk, K., Schmid, C., and Zisserman, A. (2004). Human Detection Based on a Probablistic Assembly of Robust Part Detectors. Proceedings of the 8th European Conference on Computer Vision (ECCV 2004) (eds. T. Pajdla and J. Matas), pp. 68-82, Prague, Czech Republic. ISBN 978-3-540-21981-1. [61]

Mitchell, T. M. (1997). Machine Learning. McGraw-Hill Series in Computer Science. McGraw-Hill, New York, NY. ISBN 0-07-115467-1. [77]

Mitchell, T. M. (1999). The role of Unlabeled Data in Supervised Learning. Proceedings of the Sixth International Colloquium on Cognitive Science, Springer-Verlag, New York, NY. ISBN 1-40202-057-0. [63]

Mitchell, T. M., Hutchinson, R., Niculescu, R., Pereira, F., Wang, X. R., Just, M., and Newman, S. (2004). Learning to decode cognitive states from brain images. Machine Learning, Vol. 57, pp. 145-175. ISSN 1573-0565. [104, 128]

Mohan, A., Papageorgiou, C., and Poggio, T. (2001). Example-Based Object Detection in Images by Components. IEEE Transactions on Pattern Analysis and Machine Intelligence, Vol. 23, No. 4, pp. 349-361. ISSN 0018-9340. [61, $62]$

Montgomery, D. C. and Runger, G. C. (1994). Applied Statistics and Probability for Engineers. John Wiley \& Sons, Inc., New York, NY. ISBN 0-471-54041-2. $[31]$

Moore, D. J., Essa, I. A., and Hayes, M. H. (1999). Exploiting Human Actions and Object Context for Recognition Tasks. Proceedings of the 7th IEEE International Conference on Computer Vision, Corfu, Greece. [63]

Mourão-Miranda, J., Bokde, A. L. W., Born, C., Hampel, H., and Stetter, M. (2005). Classifying brain states and determining the discriminating activation patterns: Support Vector Machine of functional MRI data. NeuroImage, Vol. 28, pp. 980-995. ISSN 1053-8119. [126]

Ogawa, S., Lee, T. M., Kay, A. R., and Tank, D. W. (1990). Brain magnetic resonance imaging with contrast dependent on blood oxygenation. Proceedings of the National Academy of Sciences, Vol. 87, pp. 9868-9872. ISSN 1091-6490. $[70]$

Paletta, L. and Greindl, C. (2003). Context Based Object Detection from Video. Proceedings of the 3rd IEEE International Conference on Computer Vision 
Systems ICVS 2003 (ed. J.L. Crowley et al.), Springer-Verlag, Heidelberg, Germany. ISBN 3-54000921-3. [62]

Palmer, S. E. (1999). Vision Science, Photons to Phenomenology. MIT Press, Cambridge, MA. ISBN 0-262-16183-4. [13, 29, 30]

Papageorgiou, C. and Poggio, T. (2000). A Trainable System for Object Detection. International Journal of Computer Vision, Vol. 38, No. 1, pp. 15-33. ISSN 1573-1405. [11, 12, 17, 20, 30, 33, 35, 36, 42]

Payne, A. and Singh, S. (2005). Indoor vs. outdoor scene classification in digital photographs. Pattern Recognition, Vol. 38, No. 10, pp. 1533-1545. ISSN 0031-3203. [63]

Pereira, F., Mason, R., Just, M., Mitchell, T. M., and Kriegeskorte, N. (2006). Decoding of semantic category information from single trial fMRI activation in response to word stimuli, using searchlight voxel selection. Poster at the 12th Annual Meeting of the Organization for Human Brain Mapping. [127]

Pham, T. V., Worring, M., and Smeulders, A. W. M. (2002). Face detection by aggregated Bayesian network classifiers. Pattern Recognition Letters, Vol. 23, pp. 451-461. ISSN 0167-8655. [15]

Pioch, N. (1994). WebMuseum. Internet site: http://www.ibiblio.org/wm/paint. $[59]$

Posner, M. I. and Peterson, S. E. (1990). The Attention System of the Human Brain. Annu. Rev. Neurosci., Vol. 13, pp. 25-43. ISSN 0147-006X. [13]

Postma, E. O. (1994). SCAN: A Neural Model of Covert Attention. Ph.D. thesis, Rijksuniversiteit Limburg, Maastricht, The Netherlands. [12]

Postma, E. O., Herik, H. J. van den, and Hudson, P. T. W. (1997). SCAN: A Scalable Neural Model of Covert Attention. Neural Networks, Vol. 10, No. 6, pp. 993-1015. ISSN 0893-6080. [12, 13]

Price, J. C. (1990). Using Spatial Context in Satellite Data to Infer Regional Scale Evapotranspiration. IEEE Transactions on Geoscience and Remote Sensing, Vol. 28, No. 5, pp. 940-948.[138]

Quinlan, J. R. (1993). C4.5: Programs for Machine Learning. The Morgan Kaufmannn Series in Machine Learning. Morgan Kaufmann, San Mateo, CA. ISBN 1-55860-238-0. [32, 39, 79]

Rifkin, R., Nadermann, M., and Moreno, P. (2001). SvmFu: A Fast, Flexible Support Vector Machine Classification Algorithm. Research abstract, MIT CBCL. [17]

Rosenberg, C., Hebert, M., and Schneiderman, H. (2005). Semi-Supervised SelfTraining of Object Detection Models. Proceedings of the Seventh IEEE Workshops on Application of Computer Vision (WACV/MOTION'05), Vol. 1, pp. 29-36, IEEE Computer Society Washington. [63] 
Schneiderman, H. and Kanade, T. (2000). A Statistical Method for 3D Object Detection Applied to Faces and Cars. IEEE Conference on Computer Vision and Pattern Recognition, IEEE. ISBN 0-7695-0662-3. [11, 12, 20]

Schneiderman, H. and Kanade, T. (2004). Object Detection Using the Statistics of Parts. International Journal of Computer Vision, Vol. 56, No. 3, pp. 151-177. ISSN 1573-1405. [29, 61]

Schölkopf, B. and Smola, A. J. (2001). Learning with Kernels: Support Vector Machines, Regularization, Optimization, and Beyond. MIT Press, 1st edition. ISBN 0-262-19475-9. [17, 30]

Shotton, J., Winn, J., Rother, C., and Criminisi, A. (2006). TextonBoost: Joint Appearance, Shape and Context Modeling for Multi-Class Object Recognition and Segmentation. Lecture Notes in Computer Science, No. 3951, pp. 1-15. ISBN 978-3-540-33836-9. [62]

Simoncelli, E. P. and Olshausen, B. A. (2001). Natural Image Statistics and Neural Representation. Vol. 24, pp. 1193-1216.[2]

Smeulders, A. W. M., Worring, M., Santini, S., Gupta, A., and Jain, R. (2000). Content-Based Image Retrieval at the End of the Early Years. IEEE Transactions on Pattern Analysis and Machine Intelligence, Vol. 22, No. 12, pp. 1349-1380. ISSN 0018-9340. [15]

Soanes, C. and Hawker, S. (eds.) (2005). Compact Oxford English Dictionary of Current English. Oxford University Press, Oxford, 3 edition. ISBN 0-19861022-X. [2]

Sung, K. K. and Poggio, T. (1998). Example-Based Learning for View-Based Human Face Detection. IEEE Transactions on Pattern Analysis and Machine Intelligence, Vol. 20, No. 1, pp. 39-51. ISSN 0018-9340. [11, 12, 20, 39]

Takerkart, S. and Daubechies, I. C. (2006). A new adaptive wavelet-thresholding scheme for the estimation of fMRI activation maps. Poster at the 12th Annual Meeting of the Organization for Human Brain Mapping. [126, 127]

Talairach, J. and Tournoux, P. (1988). Co-Planar Stereotaxic Atlas of the Human Brain. Thieme Medical Publishers, New York, NY. ISBN 0-86577-293-2. $[70]$

Tolimieri, R., An, M., and Lu, C. (1989). Algorithms for discrete fourier transform and convolution. Springer-Verlag, New York, NY. ISBN 0-387-97118-1.[152]

Torralba, A. (2002). Contextual Modulation of Target Saliency. Advances in Neural Information Processing Systems 14 (eds. T. G. Dietterich, S. Becker, and Z. Ghahramani), pp. 1303-1309, MIT Press, Cambridge, MA. ISBN 0-262-04208-8. [50, 62] 
Torralba, A. and Oliva, A. (2002). Depth Estimation from Image Structure. IEEE Transactions on Pattern Analysis and Machine Intelligence, Vol. 24, No. 9, pp. 1226-1238. ISSN 0018-9340. [31]

Torralba, A. and Oliva, A. (2003). Statistics of natural image categories. Network: Computation in Neural Systems, Vol. 14, pp. 391-412. ISSN 0954-898X. [2]

Torralba, A. and Sinha, P. (2003). Contextual Priming for Object Detection. International Journal of Computer Vision, Vol. 53, No. 2, pp. 169-191. ISSN 1573-1405. [62, 138]

Torralba, A., Murphy, K. P., Freeman, W. T., and Rubin, M. A. (2003). ContextBased Vision System for Place and Object Recognition. Proceedings of the ninth IEEE International Conference on Computer Vision, ICCV 2003, Vol. 1, pp. 273-280. ISBN 0-7695-1950-4. [62, 63]

Torralba, A., Murphy, K. P., and Freeman, W. T. (2004). Sharing features: efficient boosting procedures for multiclass object detection. Proceedings of the 2004 IEEE Computer Society Conference on Computer Vision and Pattern Recognition CVPR'04. ISBN 0-7695-2158-4. [63]

Torralba, A., Oliva, A., Castelhano, M., and Henderson, J. M. (2006). Contextual Guidance of Attention in Natural scenes: The role of Global features on object search. Psychological Review, Vol. 133, No. 4, pp. 766-786. ISSN 0033-295X. $[62,138]$

Vanello, N., Positano, V., Milanesi, M., Santarelli, M. F., Hartwig, V., Gentili, C., Ricchiardi, E., Sani, L., Pietrini, P., and Landini, L. (2006). Mutual Information Based Hierarchical Clustering of Independent Components Extracted from fMRI Data. Poster at the 12th Annual Meeting of the Organization for Human Brain Mapping. [127]

Verbeek, J. J., Vlassis, N., and Kröse, B. (2003). Efficient Greedy Learning of Gaussian Mixture Models. Neural Computation, Vol. 15, No. 2, pp. 469-485. ISSN 1530-888X. [31, 36, 37, 127]

Vinette, C., Gosselin, F., and Schyns, P. G. (2004). Spatio-Temporal Dynamics of Face Recognition in a Flash: it's in the Eyes. Cognitive Science, Vol. 28, pp. 289-301. ISSN 0364-0213. [34]

Viola, P. and Jones, M. (2001a). Robust Real-time Object Detection. Proceedings of the Second International Workshop on Statistical and Computational Theories of Vision - Modeling, Learning, Computing, and Sampling, Vancouver, Canada. $[11,12]$

Viola, P. and Jones, M. (2001b). Rapid Object Detection using a Boosted Cascade of Simple Features. Proceedings of the Conference on Computer Vision and Pattern Recognition. ISBN 0-7695-1272-0. [17, 18, 20, 22, 42, 48, 75, 127, 151, 153] 
Ward, M. D. and Gleditsch, K. S. (2002). Location, Location, Location: An MCMC Approach to Modeling the Spatial Context of War and Peace. Political Analysis, Vol. 10, No. 3, pp. 244-260. [138]

Watson, A. B. and Amahuda, A. J. (1989). A hexagonal orthogonal-oriented pyramid as a model of image representation in visual cortex. IEEE Transactions on Biomedical Engineering, Vol. 36, pp. 97-106. ISSN 0018-9294. [124]

Weeda, W. D., Huizinga, H. M., and Waldorp, L. J. (2006). Calculating fMRI activation using Activated Region Fitting. Poster at the 12th Annual Meeting of the Organization for Human Brain Mapping. [127]

Yarbus, A. L. (1967). Eye movements and vision. Plenum Press, New York, NY. $[13]$ 



\section{Appendix A}

\section{Performance considerations in Bayesian fMRI brain mapping}

In this appendix we discuss two implementation issues that largely reduce the computational complexity, and thus calculation time, of our method introduced in Chapter 6. First, we discuss the use of integral images in spherical-wavelet calculation. Second, we discuss the use of an efficient sorting algorithm.

\section{A.1 Integral images and wavelet calculation}

Our method uses an overcomplete wavelet basis on the spherical surface as features. This implies that a large number of wavelets needs to be calculated, to the point that the feature calculation becomes the bottleneck of the method as far as computational speed is concerned. In this section, we will show that the calculation of wavelets on the spherical surface can be sped up considerably, we using a modified version of the integral-image framework, (e.g., Viola and Jones (2001b), Lienhart et al. (2002)) from computer vision.

In the next three sections, we will introduce the modified integral-image framework, show how wavelets are calculated within this framework, and discuss the computational complexity of our integral-image based wavelet calculation compared to traditional wavelet-calculation methods.

\section{A.1.1 Integral-image framework}

The traditional integral-image framework was introduced into the computer-vision domain by Viola and Jones (2001b). It speeds up the calculation of wavelets in the following way. A wavelet at a given scale $s$ can be regarded in the most general sense as a 2-dimensional filter $f(x, y)$. An overcomplete decomposition, in which 
this wavelet is calculated for all positions in a 2-dimensional image $M(x, y)$, can be regarded as a 2-dimensional convolution:

$$
M(x, y) * f(x, y) \equiv \sum_{h=1}^{s} \sum_{v=1}^{s} M(x+h, y+v) f(h, v) .
$$

This operation can be implemented as a sum, as indicated in the equation above, or in the Fourier domain (Tolimieri, An, and Lu, 1989), but the computational complexity will in all cases be a function (linear of supra-linear) of the wavelet scale $s$. However, in the case of Haar (or Haar-like) wavelets often used in computer vision, the filter $f(x, y)$ is composed of s small number of areas (typically 1 to 4 ), for which the filter coefficients are equal. More specifically, the coefficients are usually either \pm 1 , or corrected for scale as $\pm 1 / s^{2}$. The fact that the filter coefficients are equal over only a few areas in the filter, can be exploited. We will briefly show how this fact is exploited in a regular rectangular 2-dimensional image, and then generalise towards the spherical surface.

In a regular 2-dimensional image, the integral image $I(x, y)$ at each point is the sum of the image-area to the upper left. That is:

$$
I(x, y) \equiv \sum_{h=1}^{x} \sum_{v=1}^{y} M(h, v)
$$

The crucial point in calculating a wavelet-region with a constant filter coefficient (i.e., $f(x, y)=f_{C}=$ constant), is the realisation that

$$
\begin{aligned}
\sum_{h=1}^{s} \sum_{v=1}^{s} M(x+h, y+v) f(h, v) & =\sum_{h=1}^{s} \sum_{v=1}^{s} M(x+h, y+v) f_{C} \\
& =f_{C} \sum_{h=1}^{s} \sum_{v=1}^{s} M(x+h, y+v)^{.} \\
& =f_{C} \times \text { Sum of image area. }
\end{aligned}
$$

In other words, we only need to be able to calculate the sum over the original image over a certain area, to be able to calculate a portion of a wavelet. If we need the sum over the image area defined by $x \in[\ell, r]$ and $y \in[t, b]$, it can be readily shown that

Sum of image area $=I(r, b)-I(r, t-1)-I(l-1, b)+I(l-1, t-1)$.

We have thus established that the sum of an image area, and thus a portion of a wavelet, can be calculated in a constant number (in this case 4) of table lookups in the integral image. This is fully independent of the wavelet scale $s$. A full Haar-like wavelet can be calculated by summing the constant-coefficient portions (usually 1 to 4 ) of which it is composed. 
The integral image itself can be calculated in a single pass through the image data, as shown by Viola and Jones (2001b).

On the spherical surface, the framework presented above needs to be modified for two reasons. First, the coordinate system on the spherical surface is not rectangular, and second, the borders of the constant-coefficient portions of our spherical wavelets, as defined in Figure 6.4, are not perpendicular to the coordinate axes.

In our modified framework, we define integral images for each of the 20 base triangles of the icosahedron of which the sphere is composed. We need three types of integral images, as shown in Figure A.1. The top left panel shows the coordinate system within the triangle. The top right panel shows the first type of integral image, $I_{I}$. The bottom left panel shows the second type of integral image, $I_{I I}$, and the bottom right panel shows the third type of integral image, $I_{I I I}$. In each panel, the lower-right point in the integral image equals to sum of the shaded area in the original image.

\section{A.1.2 Calculation of wavelets}

In this section, we will show how to calculate the wavelets types as introduced in Figure 6.4, in the integral-image framework introduced in the previous section. To this end, we show how to calculate the lower right portion of a type- 2 wavelet. Portions of other wavelets are calculated in a analogous manner.

Figure A.2 shows how to calculate the lower right portion of a type- 2 wavelet of scale 3 in four steps. Each row of figures corresponds to one step, where the left figure shows the integral-image look-up that is performed in that particular step, and the right figure shows the progress of calculating the wavelet portion.

In the first step, we perform a table look-up in the type-II integral image, at the position indicated by the large solid black circle. This results in a value that equals the sum of the entire area shown on the right-hand side.

In the second step, we perform an additional table look-up in the type-II integral image, and we subtract this value from the value we obtained in the first step. The value we have now equals the sum of the area shown on the right. Clearly, the shape of the wavelet portion starts to become visible. However, the area is still too large at the upper right end.

In the third step, we perform a table look-up in the type-III integral image, and we subtract this value from the value we obtained after the second step. This results in a value that is equal to the sum of the light area, minus the sum of the dark area, as shown in the right-hand panel of the third row in Figure A.2. Clearly, the shape of the light-coloured area now is equal to that of the lower right portion of the type- 2 wavelet. However, we still have to correct for the negative area above.

In the forth step, we perform an additional table look-up in the type-III integral image, and add this value to the value we obtained after the third step. As the value of the type-III integral image at the point indicated, is exactly equal to that of the surplus negative area obtained after the third step, the fourth step results 

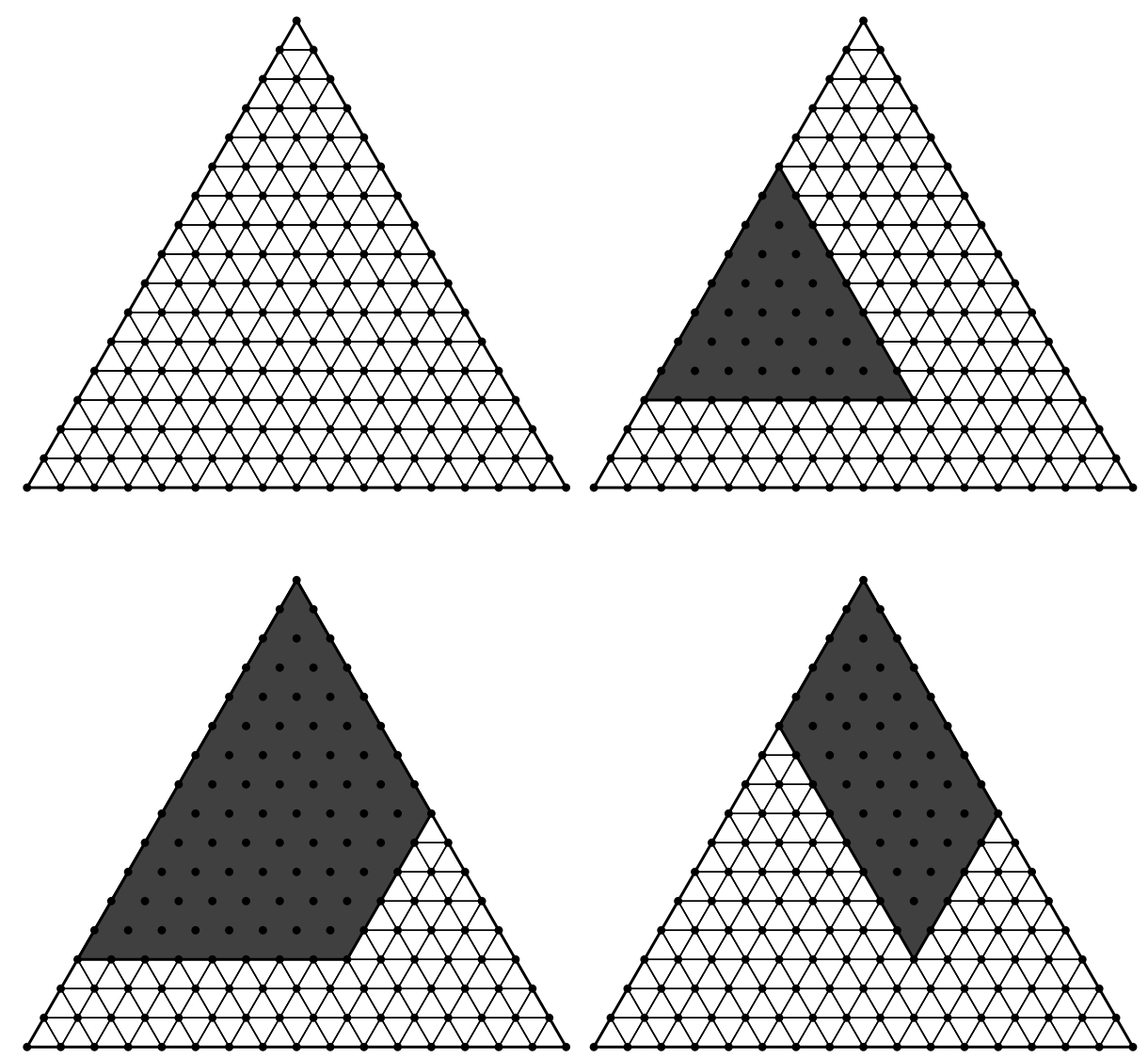

Figure A.1: Integral images, from left to right: Triangle grid, integral image type I, integral image type II, and integral image type III.

in a value that is exactly equal to that of the lower right portion of the type- 2 wavelet.

We have now shown that the lower right portion of a type- 2 wavelet can be calculated in 4 table look-ups. It should be noted that this number of table lookups is independent of the scale $s$ of the wavelet.

The observant reader will have noticed that in this particular example, we only used the type-II and type-III integral images. However, in order to be able to calculate the wavelet portions of the other wavelet types, the type-I integral image is needed as well.

Additionally, the above discussion does not explain how to extract wavelets that cross the boundary between two (or three) triangles. In order to be able to calculate these wavelets as well, we have extended the triangle structure to include parts of the neighbouring triangles. An example of an extended triangle is shown 

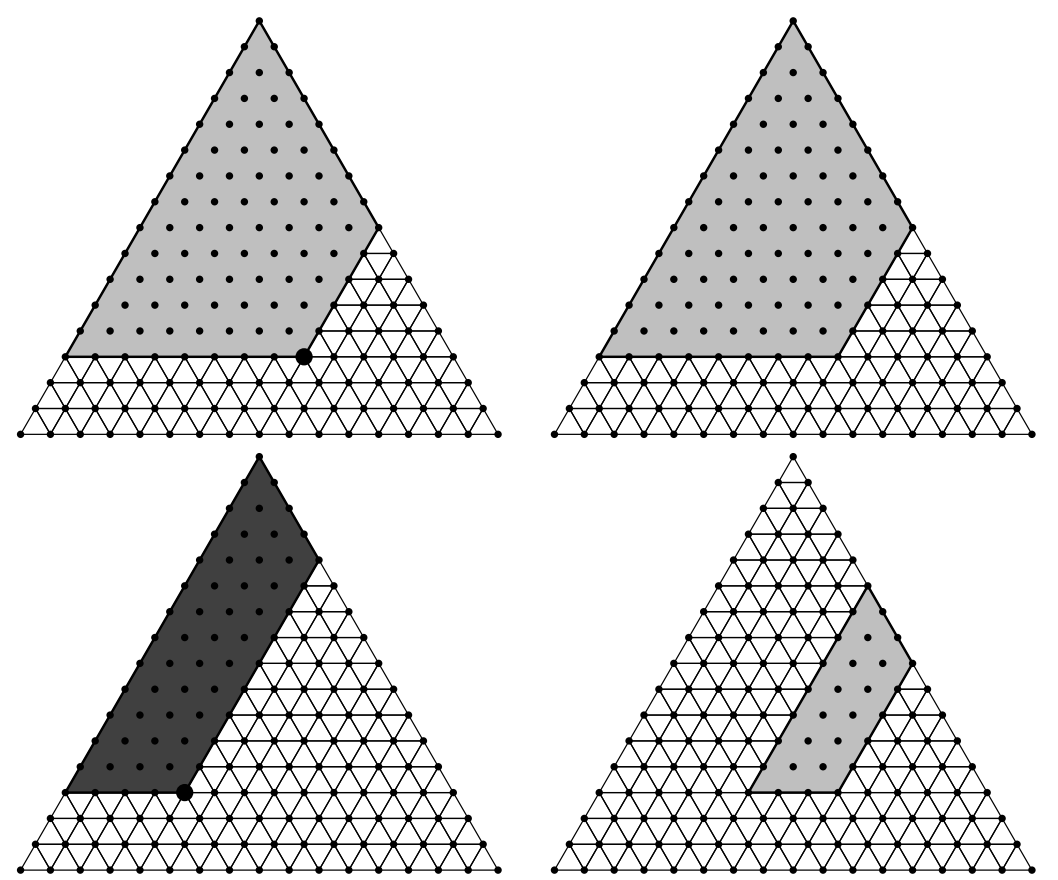

Step 1
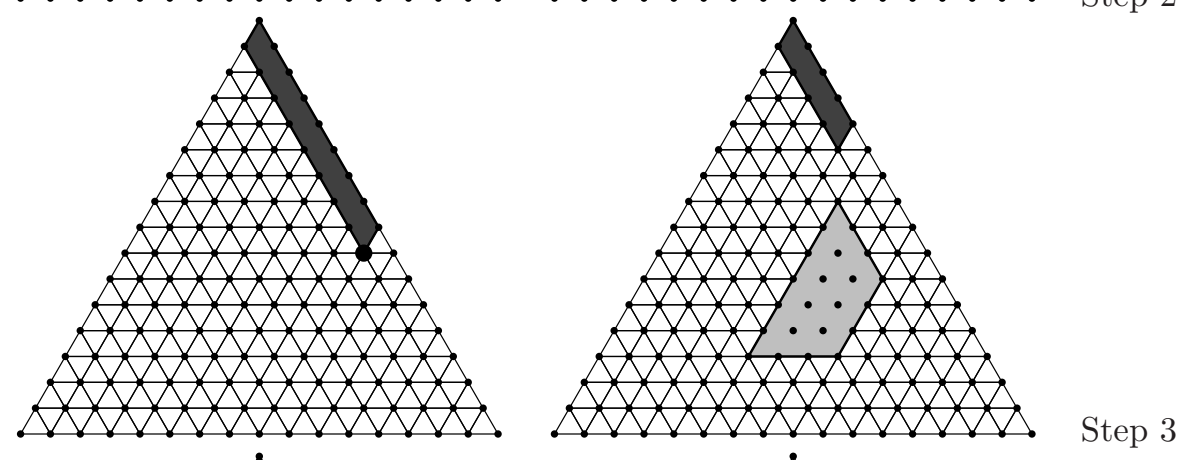

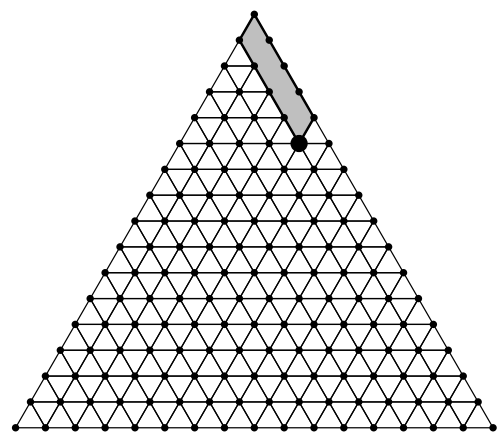

Integral-image look-up

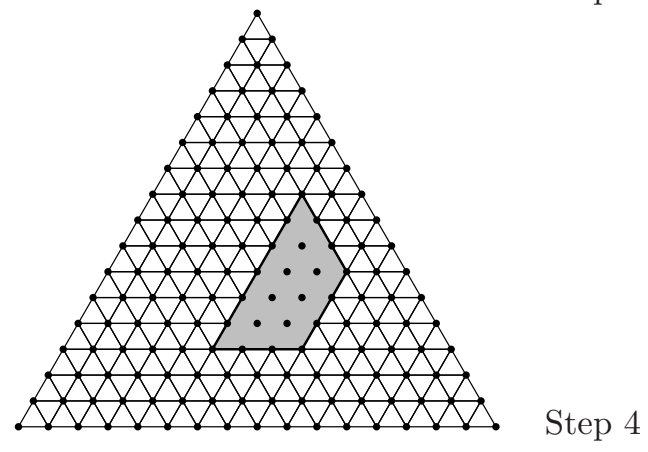

Result

Figure A.2: Calculating a portion of a type-2 wavelet in four steps 
in Figure A.3.

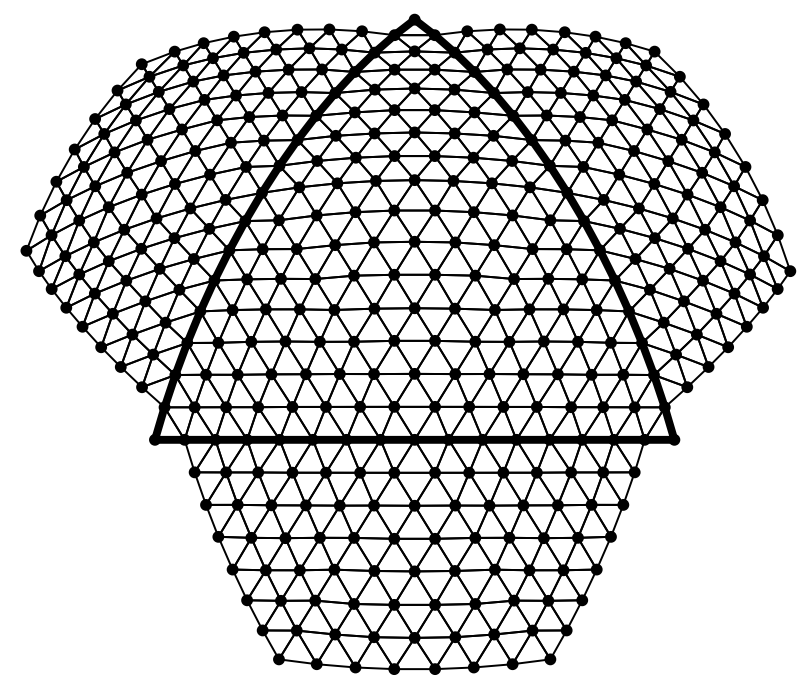

Figure A.3: Extended triangle structure for the spherical coordinate system.

\section{A.1.3 Computational complexity}

In the previous section, we have shown that we are able to calculate a wavelet of any scale on the spherical surface, in a manner that is independent of the wavelet scale, i.e., in a constant number of table look-ups. In this section, we will show that this has a significant effect on the computational complexity for the task of calculating all features, as required in our method. We will show the complexity based on an assumed square grid of $n \times n$ pixels, but the results hold for our triangular grid as well.

For a square grid of $n \times n$ pixels, where $m$ wavelets of size $s$ are to be calculated, the computational complexity is as follows (for a number of alternatives). The number of wavelets to be calculated will generally be $O\left(n^{2}\right)$. There are three manners of which all wavelet can be calculated:

First, a naive convolution implementation would sum the values of each of the $s^{2}$ locations corresponding to a scale- $s$ wavelet, times its weight. Consequently, the complexity for calculating a single wavelet is $O\left(s^{2}\right)$. For all the wavelets, the complexity is $O\left(m s^{2}\right)=O\left(n^{2} s^{2}\right)$.

Second, a separable implementation, in which the wavelet filter $W$ can be composed into a horizontal filter $h$, and a vertical filter $v$, as $W=v h^{T}$, basically converts the problem into two 1-dimensional convolutions over multiple rows or columns. The complexity to calculate all wavelets is $O(m s)=O\left(n^{2} s\right)$. 
Third, our integral-image based method is investigated. Our wavelet-calculation method proceeds in two steps. First, an integral image has to be calculated. Second, wavelets are calculated using the integral image calculated in the first step. The calculation of the integral image can be performed in a single pass through the data. The complexity of this is thus $O\left(n^{2}\right)$. The calculation of a single scale- $s$ wavelet based on the integral-image data, takes a constant number of table lookups (10 or 12, depending on the wavelet type), and is thus $O(1)$. For all wavelets, the complexity is $O(m)=O\left(n^{2}\right)$. We have thus shown that the wavelet scale $s$ is taken completely out of the complexity expression, in our method.

It should be noted that traditional wavelet extraction techniques (Mallat, 1999) extract a complete wavelet basis. In that case, the image is dyadically downsampled after each scale extraction. This does allow for an $O\left(n^{2}\right)$ extraction, but an overcomplete basis cannot be extracted this way. As we use an overcomplete basis, that technique cannot be used on our method.

\section{A.2 Sorting and grouping of specificities}

As mentioned in Subsection 6.4.2, the features are grouped by specificity, after with entire groups of features are used for classification. This sorting and grouping of specificities using a traditional sorting algorithm is of complexity $O(n \log n)$ for $n$ features. However, for $k$ samples of each stimulus, the specificities can assume only $O\left(k^{2}\right)$ distinct values. This fact can be exploited in the sorting algorithm, in order to sort the features in complexity $O(n)$.

In order to do so, we modify the standard counting-sort algorithm (Knuth, 1998) by indexing each feature by both $P\left(a_{i} \mid c_{1}\right)$ and $P\left(a_{i} \mid c_{2}\right)$. More specifically, since $P\left(c_{1}\right)=P\left(c_{2}\right)$, both $i_{1}=k P\left(a_{i} \mid c_{1}\right)$ and $i_{2}=k P\left(a_{i} \mid c_{2}\right)$ are integers on the interval $[0, k]$. We can thus assign each attribute to bin $i_{1}+i_{2}(k+1)$, and do so in a linear scan through the feature array. In practice, we allocate a temporary integer array of length $n$, in which we store $i_{1}+i_{2}(k+1)$ for each feature. In addition, we allocate a small integer array of length $(k+1)^{2}$, in which we count the number of features in each bin. Then, we perform a second linear scan through the temporary array in order to store each feature in its appropriate bin. Thus, we are able to sort and group the features in $O(n)$.

As the number of features $n$ can become rather large $\left(\sim 10^{5}\right)$ in our overcomplete wavelet basis, our modified sorting algorithm in which we bring down the complexity from $O(n \log n)$ to $O(n)$, saves considerable time. 
158 Appendix A: Performance considerations in Bayesian fMRI brain mapping 


\section{Summary}

The rapid growth of digitally stored images is accompanied by the equally fast development of image-analysis techniques. The past two decades have brought many powerful methods for image analysis, but the reliability of the techniques is still an issue. In this thesis, we combine image-analysis and machine-learning methods in an attempt to improve the reliability of image-analysis methods, by using knowledge about the structure of the images. This knowledge mostly takes the form of statistical regularities within the local spatial context of an image region of interest. In Chapter 1, we formalise the use of context in image analysis, by (1) providing a quite general definition of "image", and (2) setting sensible bounds on what we allow to be the context. This leads to the following problem statement.

Problem statement: How can information from a local spatial context be utilised to improve the performance or reliability of imageanalysis techniques?

In order to approach this problem statement in a practical manner, we selected two application domains in which we applied contextual information to improve the reliability of an image-analysis task. The two domains are (1) the cultural-heritage domain, and (2) the medical domain. The image-analysis task in the cultural-heritage domain is the detection of objects in gray-scale images. The image-analysis task in the medical domain is the detection of task-specific brain areas from functional MRI imagery. In Chapter 1 we describe both domains and tasks, and remark that the three definitions of (1) "local spatial context", (2) the nature of the information extracted from it, and (3) the measure of performance, can only be formulated well if they are specific to the application domain. Therefore, we specify our research task in the form of domain-specific problem statements, as well as domain-specific research questions.

Part I of the thesis consists of Chapters 2 to 5 , and covers the cultural-heritage domain. In Chapter 2, we describe the object-detection task in detail, and we formulate our research questions. We propose to describe object detection as a two-stage process, in which one of the stages is an object-detection stage, and the other a context-detection stage. The two stages can be ordered in a selection realisation, and a validation realisation. In the selection realisation, contextual 
information is used to select possibly interesting regions in the image, after which the object detector is used in those regions only. In the validation realisation, the object detector is used to obtain a raw set of object candidates, after which contextual information is used to validate (or invalidate) the candidates. Although the ordering of the stages does not affect the precision of a context-based method, a particular ordering can be benificial for the performance in terms of speed. Then, in the same chapter, we continue by introducing precise performance criteria for the object-detection task. We describe the datasets used to train and validate our methods. Finally, we descibe binary-classifier structures that will be used throughout the object-detection chapters. In the Chapters 3 and 4 we describe two new context-based object-detection methods.

In Chapter 3, we present the context-detection method. It is a selectionrealisation method. The idea is as follows. We train a binary classifier on image patches comprising a larger area than the object itself. This classifier is then used to find possibly interesting regions in the image. Thereafter, a standard object detector is used to inspect only these possibly interesting regions. Experimental results show that the context-detection method detects objects considerably faster than a traditional object detector. However, the detection realiability is not significantly better. We conjecture that this is caused by the fact that the variance within the class of contexts is considerably higher than within the class of objects, and that this makes the learning problem intractable. We expect that using domain knowledge on relative locations within the context would improve the performance.

As a direct consequence, in Chapter 4, we formulate the abovementioned expectation as an additional research question. Then, in relation to the new research question, we formulate a second additional research question regarding the optimal features and classifier structure to use. Subsequently, we design a second contextbased object-detection method, which uses domain knowledge on relative locations within the context. It is called the gradient method. The gradient method is a validation realisation of the context-based object detection. It consists of two stages. First, a standard object detector is used over the entire image, to obtain a set of object candidates. Then, a contextual-validation stage is used to assign a confidence to each of the candidates based on relative locations. Only candidates of which the confidence exceeds a certain threshold, are retained. Results show that the detection reliability of the gradient method is considerably better than that of a standard object detector. The small price to be paid for this improved reliability is a slightly slower detection.

In Chapter 5 we check the outcome of our expectation that the use of knowledge on relative locations would improve reliability. We consider a number of alternative reasons and start a discussion of arguments pro and con. From the discussion, we may conclude that it is not the use of relative-location knowledge per se that causes the improved reliability, but the specific manner in which we use this information causes the improved reliability. A careful use of spatial-relation information allows us to select features in an efficient manner. 
Part II of the thesis consists of Chapters 6 to 8, and covers the medical domain. In Chapter 6, we introduce the task of fMRI brain mapping in detail, and we provide a brief overview of the state of the art. We identify two main problems in the current methods: (1) the current methods disregard local spatial interaction between brain areas, and (2) the current methods require the selection of a statistical threshold to determine the size of the task-specific region. Our research attempts to solve these two problems by developing the region-based method, which uses wavelet features. We continue the chapter by providing an introduction to fMRI, and by addressing sampling issues important to our method. Subsequently, we describe the region-based method in a Bayesian framework. Our first experimental set-up consists of simulated fMRI activations on a background of measured resting-state noise. The use of simulated data allows us to explore the range of the circumstances in which our method is useful, and what limits there are to its applicability. The results on the simulated data allow us to draw two conclusions. First, we may conclude that the region-based method yields a similar accuracy in determining task-specific regions compared to a traditional method under high-contrast conditions, while it outperforms the traditional method under low-contrast conditions. Second, we may conclude that the region-based method is capable of estimating a task-specific region scale without the need for setting a statistical threshold. The region-based method thus solves the two major problems identified in the traditional method. It does so for simulated data. Therefore, we challenge the use of simulated data, and propose to use real data.

In Chapter 7, we apply the region-based method to real fMRI data from a group of subjects in a visual task that discriminates between faces and objects. First, the dataset is described in detail; the algorithmic differences between the use of simulated data and real data are extensively discussed; and the performance criteria are defined. Then, the experiments are performed, and the results are presented. From the results and the discussion on the results, we may draw two main conclusions. First, we may conclude that the region-based method outperforms the traditional method in the accuracy of task-specific region detection, and it yields useful scale estimates of the regions. This implies that our method generalises well from simulated data to real data. Second, we may conclude that our method is significantly more robust against inter-personal anatomical differences than the traditional method.

In Chapter 8, we adopt a more high-level view of the region-based method, and discuss noise and feature issues. In addition, we discuss (1) the use of Bayesian classification in our method, and (2) the connections between our method and related work. Finally, we address future directions for research and possible extensions to our method.

In Chapter 9, we provide our final conclusions on the work presented in this thesis. So, we return to the research questions stated in Chapter 1, and answer them. Given these answers, we subsequently answer the problem statement. In brief, the use of information from the local spatial context has proven to be beneficial to the performance in two image-analysis domains. More particularly, the 
specific manner of using spatial relations within the context to reduce the feature space, has proven to be the main enabling point. We complete the chapter and the book by formulating future research topics and new application goals. 


\section{Samenvatting}

De snelle groei in digitaal opgeslagen afbeeldingen is gepaard gegaan met een even snelle groei in beeldanalysetechnieken. In de afgelopen twee decennia zijn veel krachtige beeldanalysemethoden ontwikkeld, maar de betrouwbaarheid van deze methoden laat nog te wensen over. In dit proefschrift combineren we beeldanalyseen machineleermethoden in een poging om de betrouwbaarheid van beeldanalysemethoden te verbeteren, door gebruik te maken van kennis over de structuur van afbeeldingen. Deze kennis bestaat in het algemeen uit statistische regelmatigheden binnen de locale spatiële context van een interessant gedeelte in een afbeelding. In Hoofdstuk 1 formaliseren we het gebruik van context door (1) een vrij afgemene definitie van "afbeelding" te geven en (2) zinnige grenzen te stellen aan wat het begrip context mag inhouden. Dit leidt tot de volgende probleemstelling.

Probleemstelling: Hoe kan informatie uit een locale spatiële context worden gebruikt om de prestaties of betrouwbaarheid van beeldanalysetechnieken te verbeteren?

Om deze probleemstelling op praktische wijze te benaderen, hebben we twee toepassingsdomeinen geselecteerd, waarin we contextuele informatie toepassen om de betrouwbaarheid van een beeldanalysetaak te verbeteren. De twee domeinen zijn (1) het cultureel-erfgoed domein en (2) het medisch domein. De beeldanalysetaak in het cultureel-erfgoed domein is het detecteren van objecten in grijswaardenafbeeldingen. De beeldanalysetaak in het medisch domein is het detecteren van taakspecifieke hersengedeelten vanuit functionele-MRI beelden. In Hoofdstuk 1 beschrijven we beide domeinen en taken, en merken we op dat de drie definities van (1) "locale spatiële context", (2) de aard van de daaruit gehaalde informatie en (3) de prestatiemaat, specifiek zijn voor het toepassingsdomein. Daarom formuleren we domein-specifieke probleemstellingen, evenals domein-specifieke onderzoeksvragen.

Deel I van het proefschrift bestaat uit de Hoofdstukken 2 tot en met 5, en beslaat het cultureel-erfgoed domein. In Hoofdstuk 2 beschrijven we de objectdetectietaak in detail en formuleren we onze onderzoeksvragen. We stellen voor om objectdetectie op te vatten als een proces dat uit twee stadia bestaat, waarin één van de stadia een objectdetectiestadium is, en het andere een contextdetectiestadium. Op basis van hun volgorde kunnen de twee stadia in een selectierealisatie en een validatierealisatie worden gerangschikt. In de selectierealisatie wordt contextuele informatie gebruikt om mogelijk interessante gebieden in een afbeelding 
te selecteren, waarna de objectdetector alleen in deze gebieden wordt gebruikt. In de validatierealisatie wordt de objectdetector gebruikt om een verzameling ruwe objectcandidaten te verkrijgen, waarna contextuele informatie wordt gebruikt om deze candidaten te (in)valideren. Hoewel de volgorde van de stadia geen invloed heeft op prestaties van een contextgebaseerde methode, kan de keuze voor een bepaalde volgorde wel voordelen op het gebied van snelheid opleveren. We zetten Hoofdstuk 2 voort met een beschrijving van precieze prestatiematen voor de objectdetectietaak. We beschrijven de dataverzamelingen die zijn gebruikt om onze methoden te trainen en valideren. Tenslotte beschrijven we de binaire-classificatie structuren diue in het gehele objectdetectiegedeelte van dit proefschrift worden gebruikt. In de Hoofdstukken 3 en 4 beschrijven we twee nieuwe contextbaseerde objectdetectiemethoden.

In Hoofdstuk 3 beschrijven we contextdetectiemethode. Dit is een methode op basis van selectierealisatie. Het idee is als volgt. We trainen een binaire classificator op afbeeldingsgedeelten die een groter gebied dan het object zelf omvatten. Deze classificator wordt vervolgens gebruikt om mogelijk interessante gebieden in een afbeelding te vinden. Daarna wordt een standaard objectdetector gebruikt om alleen binnen deze mogelijk interessante gebieden te zoeken. Experimentele resultaten laten zien dat de contextdetectiemethode aanzienlijk sneller is in het detecteren van objecten dan een standaard objectdetector. De betrouwbaarheid is echter niet significant beter. We nemen aan dat dit wordt veroorzaakt doordat de variantie binnen de klasse van contexten aanzienlijk groter is dan die binnen de klasse van objecten, en dat dit het leerprobleem moeilijk hanteerbaar maakt. We verwachten dat de prestaties kunnen worden verbeterd door domeinkennis over relatieve posities binnen de context te gebruiken.

Bijgevolg formuleren we in Hoofdstuk 4 bovenstaande verwachtingen als een extra onderzoeksvraag. In relatie met deze nieuwe onderzoeksvraag formuleren we bovendien een tweede extra onderzoeksvraag aangaande de optimale kenmerkenverzameling en classificatiestructuur. We ontwerpen een tweede contextgebaseerde objectdetectiemethode die gebruik maakt van relatieve locaties binnen de context. Deze methode heet de gradiëntmethode. De gradiëntmethode is een validatierealisatie van contextgebaseerde objectdetectie, en bestaat uit twee stadia. Op de eerste plaats wordt een standaard objectdetector gebruikt binnen de gehele afbeelden om zodoende een verzameling ruwe objectcandidaten te verkrijgen. Vervolgens wordt een contextvalidatiestadium gebruikt om aan elk van de candidaten, op basis van relatieve locaties, een betrouwbaarheid toe te kennen. Alleen candidaten met een voldoend hoge betrouwbaarheid blijven over. Resultaten laten zien dat de detectiebetrouwbaarheid van de gradiëntmethode aanzienlijk beter is dan die van een standaard objectdetector. De lage prijs die daarvoor betaald moet worden leidt tot een iets langzamere detectie.

In Hoofdstuk 5 komen we terug op onze verwachting dat het gebruik van kennis over relatieve locaties de betrouwbaarheid zou verbeteren. We nemen een aantal alternatieve redenen in ogenschouw, en voeren een discussie met ruimte voor argumenten voor en de argumenten tegen. Uit deze discussie mogen we concluderen dat de verbeterde betrouwbaarheid niet wordt veroorzaakt door het gebruik van relatieve locatie-informatie op zichzelf, maar door de specifieke manier waarop we 
deze informatie gebruiken. Een weloverwogen gebruik van informatie over spatiële relaties stelt ons namelijk in staat om op efficiente wijze kenmerken te selecteren.

Deel II van het proefschrift bestaat uit de Hoofdstukken 6 tot en met 8, en houdt zich bezig met het medisch domein. In Hoofdstuk 6 beschrijven we de $f M$ $R I$ brain mapping taak in detail, en geven we een kort overzicht van de stand van zaken in dit onderzoeksgebied. We identificeren twee belangrijke problemen in bestaande methoden: (1) bestaande methoden houden geen rekening met spatiële interacties tussen verschillende gedeelten van de hersenen en (2) in bestaande methoden moet een statistische drempel worden gekozen om de grootte van taakspecifieke hersengedeelten te bepalen. In ons onderzoek proberen we beide problemen op te lossen door een regiogebaseerde methode op basis van waveletkenmerken te ontwikkelen. We zetten het hoofdstuk voort met een introductie van de fMRI-techniek, en met een beschrijving van bemonsteringskwesties die voor onze methode van belang zijn. Vervolgens beschrijven we de regiogebaseerde methode in een Bayesiaans kader. Onze eerste experimentele opstelling bestaat uit gesimuleerde fMRI-activaties bovenop een achtergrond van ruis die is gemeten bij een proefpersoon in rust. Het gebruik van gesimuleerde data stelt ons in staat de grenzen op te zoeken van de omstandigheden waarin de methode nuttig is. We kunnen op basis van de resultaten van de gesimuleerde data twee conclusies trekken. Op de eerste plaats levert de regiogebaseerde methode, onder omstandigheden met een hoog contrast, een vergelijkbare detectiebetrouwbaarheid als een standaardmethode. De betrouwbaarheid onder omstandigheden met weinig contrast is beter dan die van de standaardmethode. Op de tweede plaats is de regiogebaseerde methode in staat om een goede schaalschatting te maken van taakspecifieke gebieden, zonder dat daarvoor de selectie van een statistische drempel nodig is. Zodoende lost de regiogebaseerde methode beide problemen van de traditionele methode op. Dit is het geval voor gesimuleerde data. We stellen daarom het gebruik van gesimuleerde data ter discussie, en stellen voor om echte data te gebruiken.

In Hoofdstuk 7 passen we de regiogebaseerde methode toe op echte fMRI-data uit een visueel experiment waarin een groep proefpersonen onderscheid maakte tussen gezichten en objecten. Eerst wordt de dataverzameling uitgebreid beschreven, worden de algoritmeverschillen tussen het gebruik van gesimuleerde en echte data beschreven, en worden prestatiematen gedefinieerd. Vervolgens worden de experimenten uitgevoerd, en de resultaten gepresenteerd en bediscussieerd. Op basis van de resultaten en de discussie kunnen we twee belangrijke conclusies trekken. Op de eerste plaats levert de regiogebaseerde methode een betrouwbaarder detectie van taakspecieke hersengebieden dan de traditionele methode. Dit impliceert dat onze methode goed generaliseert van gesimuleerde naar echte data. Op de tweede plaats is onze methode significant robuuster tegen interpersoonlijke anatomische verschillen dan de traditionele methode.

In Hoofdstuk 8 bezien we de regiogebaseerde methode vanuit een algemener perspectief. Op de eerste plaats discussiëren we over ruis- en kenmerkkwesties. Vervolgens discussiëren we over (1) het gebruik van Bayesiaanse classificatie in 
onze methode en (2) de relatie tussen onze methode en bestaand onderzoek. Tenslotte geven we toekomstige onderzoeksrichtingen aan, en stellen we mogelijke uitbreidingen op onze methode voor.

In Hoofdstuk 9 trekken we de uiteindelijke conclusies uit het werk dat in dit proefschrift is beschreven. Daartoe komen we terug op de onderzoeksvragen die we in Hoofdstuk 1 stelden, en beantwoorden deze. Op basis van deze antwoorden beantwoorden we vervolgens de probleemstelling. Kort samengevat is het gebruik van informatie uit de locale spatiële context voordelig gebleken voor de prestaties in twee beeldanalysedomeinen. In het bijzonder is de specifieke wijze waarop het gebruik van spatiële relaties binnen de context een reductie van de kenmerkenruimte mogelijk maakt, de belangrijkste oorzaak voor de verbeterde prestaties gebleken. We besluiten het hoofdstuk en het proefschrift met het formuleren van toekomstige onderzoeksonderwerpen en nieuwe toepassingsdoelen. 


\section{Curriculum Vitae}

Niek Bergboer was born in Hengelo, in the province of Overijssel, The Netherlands, on May 25th, 1977. In 1995 he started his undergraduate study in Applied Physics at the University of Twente. In 2001, he successfully defended his thesis in the field of System Identification, and received his M.Sc. degree cum laude. After graduation, he worked as researcher at the University of Twente for one year in the field of numerical algorithms in system idenfication.

Between 2002 and 2006, he worked as junior researcher (promovendus) at the Maastricht ICT Competence Centre (MICC) - Institute for Knowledge and Agent Technology (IKAT), Universiteit Maastricht, The Netherlands. The research presented in this thesis was performed during this period.

Currently, he works as a software engineer at Google, in Zürich, Switzerland. 



\section{SIKS Dissertation Series}

\section{8}

1 Johan van den Akker $\left(\mathrm{CWI}^{1}\right)$ DEGAS - An Active, Temporal Database of $A u-$ tonomous Objects

2 Floris Wiesman (UM) Information Retrieval by Graphically Browsing Meta-Information

3 Ans Steuten (TUD) A Contribution to the Linguistic Analysis of Business Conversations within the Language/Action Perspective

4 Dennis Breuker (UM) Memory versus Search in Games

5 Eduard W. Oskamp (RUL) Computerondersteuning bij Straftoemeting

\section{9}

1 Mark Sloof (VU) Physiology of Quality Change Modelling; Automated Modelling of Quality Change of Agricultural Products

2 Rob Potharst (EUR) Classification using Decision Trees and Neural Nets

3 Don Beal (UM) The Nature of Minimax Search

4 Jacques Penders (UM) The Practical Art of Moving Physical Objects

5 Aldo de Moor (KUB) Empowering Communities: A Method for the Legitimate User-Driven Specification of Network Information Systems

6 Niek J.E. Wijngaards (VU) Re-Design of Compositional Systems

7 David Spelt (UT) Verification Support for Object Database Design

8 Jacques H.J. Lenting (UM) Informed Gambling: Conception and Analysis of a Multi-Agent Mechanism for Discrete Reallocation

\section{0}

1 Frank Niessink (VU) Perspectives on Improving Software Maintenance

2 Koen Holtman (TU/e) Prototyping of CMS Storage Management

3 Carolien M.T. Metselaar (UvA) Sociaal-organisatorische Gevolgen van Kennistechnologie; een Procesbenadering en Actorperspectief

\footnotetext{
${ }^{1}$ Abbreviations: SIKS - Dutch Research School for Information and Knowledge Systems; CWI - Centrum voor Wiskunde en Informatica, Amsterdam; EUR - Erasmus Universiteit, Rotterdam; KUB - Katholieke Universiteit Brabant, Tilburg; KUN - Katholieke Universiteit Nijmegen; RUL - Rijksuniversiteit Leiden; TUD - Technische Universiteit Delft; TU/e - Technische Universiteit Eindhoven; UL - Universiteit Leiden; UM - Universiteit Maastricht; UT - Universiteit Twente, Enschede; UU - Universiteit Utrecht; UvA - Universiteit van Amsterdam; UvT - Universiteit van Tilburg; VU - Vrije Universiteit, Amsterdam.
} 
4 Geert de Haan (VU) ETAG, A Formal Model of Competence Knowledge for User Interface Design

5 Ruud van der Pol (UM) Knowledge-Based Query Formulation in Information Retrieval

6 Rogier van Eijk (UU) Programming Languages for Agent Communication

7 Niels Peek (UU) Decision-Theoretic Planning of Clinical Patient Management

8 Veerle Coupé (EUR) Sensitivity Analyis of Decision-Theoretic Networks

9 Florian Waas (CWI) Principles of Probabilistic Query Optimization

10 Niels Nes (CWI) Image Database Management System Design Considerations, Algorithms and Architecture

11 Jonas Karlsson (CWI) Scalable Distributed Data Structures for Database Management

\section{1}

1 Silja Renooij (UU) Qualitative Approaches to Quantifying Probabilistic Networks

2 Koen Hindriks (UU) Agent Programming Languages: Programming with Mental Models

3 Maarten van Someren (UvA) Learning as Problem Solving

4 Evgueni Smirnov (UM) Conjunctive and Disjunctive Version Spaces with InstanceBased Boundary Sets

5 Jacco van Ossenbruggen (VU) Processing Structured Hypermedia: A Matter of Style

6 Martijn van Welie (VU) Task-Based User Interface Design

7 Bastiaan Schonhage (VU) Diva: Architectural Perspectives on Information Visualization

8 Pascal van Eck (VU) A Compositional Semantic Structure for Multi-Agent Systems Dynamics

9 Pieter Jan 't Hoen (RUL) Towards Distributed Development of Large ObjectOriented Models, Views of Packages as Classes

10 Maarten Sierhuis (UvA) Modeling and Simulating Work Practice BRAHMS: a Multiagent Modeling and Simulation Language for Work Practice Analysis and Design

11 Tom M. van Engers (VU) Knowledge Management: The Role of Mental Models in Business Systems Design

\section{2}

1 Nico Lassing (VU) Architecture-Level Modifiability Analysis

2 Roelof van Zwol (UT) Modelling and Searching Web-based Document Collections

3 Henk Ernst Blok (UT) Database Optimization Aspects for Information Retrieval

4 Juan Roberto Castelo Valdueza (UU) The Discrete Acyclic Digraph Markov Model in Data Mining

5 Radu Serban (VU) The Private Cyberspace Modeling Electronic Environments Inhabited by Privacy-Concerned Agents

6 Laurens Mommers (UL) Applied Legal Epistemology; Building a Knowledge-based Ontology of the Legal Domain

7 Peter Boncz (CWI) Monet: A Next-Generation DBMS Kernel For Query-Intensive Applications 
8 Jaap Gordijn (VU) Value Based Requirements Engineering: Exploring Innovative E-Commerce Ideas

9 Willem-Jan van den Heuvel (KUB) Integrating Modern Business Applications with Objectified Legacy Systems

10 Brian Sheppard (UM) Towards Perfect Play of Scrabble

11 Wouter C.A. Wijngaards (VU) Agent Based Modelling of Dynamics: Biological and Organisational Applications

12 Albrecht Schmidt (UvA) Processing XML in Database Systems

13 Hongjing Wu (TU/e) A Reference Architecture for Adaptive Hypermedia Applications

14 Wieke de Vries (UU) Agent Interaction: Abstract Approaches to Modelling, Programming and Verifying Multi-Agent Systems

15 Rik Eshuis (UT) Semantics and Verification of UML Activity Diagrams for Workflow Modelling

16 Pieter van Langen (VU) The Anatomy of Design: Foundations, Models and Applications

17 Stefan Manegold (UvA) Understanding, Modeling, and Improving Main-Memory Database Performance

\section{3}

1 Heiner Stuckenschmidt (VU) Ontology-Based Information Sharing in Weakly Structured Environments

2 Jan Broersen (VU) Modal Action Logics for Reasoning About Reactive Systems

3 Martijn Schuemie (TUD) Human-Computer Interaction and Presence in Virtual Reality Exposure Therapy

4 Petkovic (UT) Content-Based Video Retrieval Supported by Database Technology

5 Jos Lehmann (UvA) Causation in Artificial Intelligence and Law - A Modelling Approach

6 Boris van Schooten (UT) Development and Specification of Virtual Environments

7 Machiel Jansen (UvA) Formal Explorations of Knowledge Intensive Tasks

8 Yong-Ping Ran (UM) Repair-Based Scheduling

9 Rens Kortmann (UM) The Resolution of Visually Guided Behaviour

10 Andreas Lincke (UT) Electronic Business Negotiation: Some Experimental Studies on the Interaction between Medium, Innovation Context and Cult

11 Simon Keizer (UT) Reasoning under Uncertainty in Natural Language Dialogue using Bayesian Networks

12 Roeland Ordelman (UT) Dutch Speech Recognition in Multimedia Information Retrieval

13 Jeroen Donkers (UM) Nosce Hostem - Searching with Opponent Models

14 Stijn Hoppenbrouwers (KUN) Freezing Language: Conceptualisation Processes across ICT-Supported Organisations

15 Mathijs de Weerdt (TUD) Plan Merging in Multi-Agent Systems

16 Menzo Windhouwer (CWI) Feature Grammar Systems - Incremental Maintenance of Indexes to Digital Media Warehouse

17 David Jansen (UT) Extensions of Statecharts with Probability, Time, and Stochastic Timing 
18 Levente Kocsis (UM) Learning Search Decisions

\section{4}

1 Virginia Dignum (UU) A Model for Organizational Interaction: Based on Agents, Founded in Logic

2 Lai Xu (UvT) Monitoring Multi-party Contracts for E-business

3 Perry Groot (VU) A Theoretical and Empirical Analysis of Approximation in Symbolic Problem Solving

4 Chris van Aart (UvA) Organizational Principles for Multi-Agent Architectures

5 Viara Popova (EUR) Knowledge Discovery and Monotonicity

6 Bart-Jan Hommes (TUD) The Evaluation of Business Process Modeling Techniques

7 Elise Boltjes (UM) Voorbeeld VG $_{G}$ Onderwijs; Voorbeeldgestuurd Onderwijs, een Opstap naar Abstract Denken, vooral voor Meisjes

8 Joop Verbeek (UM) Politie en de Nieuwe Internationale Informatiemarkt, Grensregionale Politiële Gegevensuitwisseling en Digitale Expertise

9 Martin Caminada (VU) For the Sake of the Argument; Explorations into Argumentbased Reasoning

10 Suzanne Kabel (UvA) Knowledge-rich Indexing of Learning-objects

11 Michel Klein (VU) Change Management for Distributed Ontologies

12 The Duy Bui (UT) Creating Emotions and Facial Expressions for Embodied Agents

13 Wojciech Jamroga (UT) Using Multiple Models of Reality: On Agents who Know how to Play

14 Paul Harrenstein (UU) Logic in Conflict. Logical Explorations in Strategic Equilibrium

15 Arno Knobbe (UU) Multi-Relational Data Mining

16 Federico Divina (VU) Hybrid Genetic Relational Search for Inductive Learning

17 Mark Winands (UM) Informed Search in Complex Games

18 Vania Bessa Machado (UvA) Supporting the Construction of Qualitative Knowledge Models

19 Thijs Westerveld (UT) Using generative probabilistic models for multimedia retrieval

20 Madelon Evers (Nyenrode) Learning from Design: facilitating multidisciplinary design teams

\section{5}

1 Floor Verdenius (UVA) Methodological Aspects of Designing Induction-Based Applications

2 Erik van der Werf (UM) AI techniques for the game of Go

3 Franc Grootjen (RUN) A Pragmatic Approach to the Conceptualisation of Language

4 Nirvana Meratnia (UT) Towards Database Support for Moving Object data

5 Gabriel Infante-Lopez (UVA) Two-Level Probabilistic Grammars for Natural Language Parsing

6 Pieter Spronck (UM) Adaptive Game AI 
7 Flavius Frasincar (TUE) Hypermedia Presentation Generation for Semantic Web Information Systems

8 Richard Vdovjak (TUE) A Model-driven Approach for Building Distributed Ontologybased Web Applications

9 Jeen Broekstra (VU) Storage, Querying and Inferencing for Semantic Web Languages

10 Anders Bouwer (UVA) Explaining Behaviour: Using Qualitative Simulation in Interactive Learning Environments

11 Elth Ogston (VU) Agent Based Matchmaking and Clustering - A Decentralized Approach to Search

12 Csaba Boer (EUR) Distributed Simulation in Industry

13 Fred Hamburg (UL) Een Computermodel voor het Ondersteunen van Euthanasiebeslissingen

14 Borys Omelayenko (VU) Web-Service configuration on the Semantic Web; Exploring how semantics meets pragmatics

15 Tibor Bosse (VU) Analysis of the Dynamics of Cognitive Processes

16 Joris Graaumans (UU) Usability of XML Query Languages

17 Boris Shishkov (TUD) Software Specification Based on Re-usable Business Components

18 Danielle Sent (UU) Test-selection strategies for probabilistic networks

19 Michel van Dartel (UM) Situated Representation

20 Cristina Coteanu (UL) Cyber Consumer Law, State of the Art and Perspectives

21 Wijnand Derks (UT) Improving Concurrency and Recovery in Database Systems by Exploiting Application Semantics

\section{6}

1 Samuil Angelov (TUE) Foundations of B2B Electronic Contracting

2 Cristina Chisalita (VU) Contextual issues in the design and use of information technology in organizations

3 Noor Christoph (UVA) The role of metacognitive skills in learning to solve problems

4 Marta Sabou (VU) Building Web Service Ontologies

5 Cees Pierik (UU) Validation Techniques for Object-Oriented Proof Outlines

6 Ziv Baida (VU) Software-aided Service Bundling - Intelligent Methods \& Tools for Graphical Service Modeling

7 Marko Smiljanic (UT) XML schema matching - balancing efficiency and effectiveness by means of clustering

8 Eelco Herder (UT) Forward, Back and Home Again - Analyzing User Behavior on the Web

9 Mohamed Wahdan (UM) Automatic Formulation of the Auditor's Opinion

10 Ronny Siebes (VU) Semantic Routing in Peer-to-Peer Systems

11 Joeri van Ruth (UT) Flattening Queries over Nested Data Types

12 Bert Bongers (VU) Interactivation - Towards an e-cology of people, our technological environment, and the arts

13 Henk-Jan Lebbink (UU) Dialogue and Decision Games for Information Exchanging Agents 
14 Johan Hoorn (VU) Software Requirements: Update, Upgrade, Redesign - towards a Theory of Requirements Change

15 Rainer Malik (UU) CONAN: Text Mining in the Biomedical Domain

16 Carsten Riggelsen (UU) Approximation Methods for Efficient Learning of Bayesian Networks

17 Stacey Nagata (UU) User Assistance for Multitasking with Interruptions on a Mobile Device

18 Valentin Zhizhkun (UVA) Graph transformation for Natural Language Processing

19 Birna van Riemsdijk (UU) Cognitive Agent Programming: A Semantic Approach

20 Marina Velikova (UvT) Monotone models for prediction in data mining

21 Bas van Gils (RUN) Aptness on the Web

22 Paul de Vrieze (RUN) Fundaments of Adaptive Personalisation

23 Ion Juvina (UU) Development of Cognitive Model for Navigating on the Web

24 Laura Hollink (VU) Semantic Annotation for Retrieval of Visual Resources

25 Madalina Drugan (UU) Conditional log-likelihood MDL and Evolutionary MCMC

26 Vojkan Mihajlovic (UT) Score Region Algebra: A Flexible Framework for Structured Information Retrieval

27 Stefano Bocconi (CWI) Vox Populi: generating video documentaries from semantically annotated media repositories

28 Borkur Sigurbjornsson (UVA) Focused Information Access using XML Element Retrieval

\section{7}

1 Kees Leune (UvT) Access Control and Service-Oriented Architectures

2 Wouter Teepe (RUG) Reconciling Information Exchange and Confidentiality: A Formal Approach

3 Peter Mika (VU) Social Networks and the Semantic Web

4 Jurriaan van Diggelen (UU) Achieving Semantic Interoperability in Multi-agent Systems: a dialogue-based approach

5 Bart Schermer (UL) Software Agents, Surveillance, and the Right to Privacy: a Legislative Framework for Agent-enabled Surveillance

6 Gilad Mishne (UVA) Applied Text Analytics for Blogs

7 Natasa Jovanovic (UT) To Whom It May Concern - Addressee Identification in Face-to-Face Meetings

8 Mark Hoogendoorn (VU) Modeling of Change in Multi-Agent Organizations

9 David Mobach (VU) Agent-Based Mediated Service Negotiation

10 Huib Aldewereld (UU) Autonomy vs. Conformity: an Institutional Perspective on Norms and Protocols

11 Natalia Stash (TUE) Incorporating Cognitive/Learning Styles in a General-Purpose Adaptive Hypermedia System

12 Marcel van Gerven (RUN) Bayesian Networks for Clinical Decision Support: A Rational Approach to Dynamic Decision-Making under Uncertainty

13 Rutger Rienks (UT) Meetings in Smart Environments; Implications of Progressing Technology

14 Niek Bergboer (UM) Context-Based Image Analysis 


\title{
Stellingen
}

\author{
behorende bij het proefschrift \\ Context-Based Image Analysis \\ Niek Bergboer, 10 oktober 2007
}

1. Bij de automatische detectie van objecten leidt het gebruik van de lokale spatiële context tot een aanzienlijke snelheidswinst.

- Dit proefschrift, hoofdstuk 3

2. Het benutten van de lokale spatiële context ter verkleining van de kenmerkruimte leidt tot een robuustere objectdetectie.

- Dit proefschrift, hoofdstukken 4 en 5.

3. De combinatie van wavelets en machine learning in fMRI brain mapping maakt het mogelijk om de omvang te schatten van een gebied dat specifiek is voor het verwerken van een bepaalde stimulus.

- Dit proefschrift, hoofdstuk 6 .

4. Het gebruik van contextuele informatie in fMRI brain mapping levert een grotere robuustheid op ten aanzien van interpersoonlijke verschillen in hersenanatomie dan traditionele methoden, die deze informatie niet gebruiken.

- Dit proefschrift, hoofdstuk 7 .

5. De beschikbaarheid van slechts weinig voorbeelden, gecombineerd met een grote kenmerkenruimte, is karakteristiek voor veel praktijkproblemen. De grootste uitdaging voor machine learning in de komende decennia bestaat daarom in het intelligent verkleinen van de kenmerkenruimte door het incorporeren van domeinkennis.

6. Het creëren van goede geannoteerde dataverzamelingen dient in zowel de planning als financiering van machine learning onderzoek een prominente plaats te krijgen.

7. Implementatiedetails van machine learning-algoritmen bepalen in belangrijke mate de prestaties. Daarom dienen ze een essentieel onderdeel te vormen van de beoordeling van wetenschappelijke publicaties.

8. De trend van het afgelopen decennium om machine learning-technieken te demystificeren door statistische formalisatie, biedt hoop op een brede acceptatie van deze technieken in de industrie.

9. Het Nederlandse financieringsbeleid ten aanzien van promovendi heeft zijn parallel in de Bootstrap Aggregation-methode uit de artificiële intelligentie. In beide wordt gepoogd goede prestaties te verkrijgen door het combineren van veel zwakke deskundigen in elkaar sterk overlappende onderwerpen. 
10. Het toenemend gebruik van Digital Restriction Management bedreigt de toegankelijkheid van het cultureel erfgoed.

11. De cumulatieve kans om door een terroristische aanslag om het leven te komen (1:88000), is in de orde van 1000 maal zo klein als de cumulatieve kans om door een verkeersongeval om het leven te komen (1:78), en zelfs nog kleiner dan de cumulatieve kans om door een insectenbeet om het leven te komen ${ }^{2}$ (1:56 789). Enige politieke bezinning op het punt van terrorismewetgeving is daarom op zijn plaats.

12. Of men rijden in een file een probleem vindt hangt in belangrijke mate af van het aantal wielen waarop men zich voortbeweegt.

\footnotetext{
${ }^{2}$ Bronnen: National Safety Counsil, Centre for Disease Control. De data betreffen de Verenigde Station over de periode 2000-2003.
} 